WHC-EP-0829

UC-510

\title{
Estimation and Characterization of Decontamination and Decommissioning Solid Waste Expected from the Plutonium Finishing Plant
}

\author{
J. S. Millar \\ J. A. Pottmeyer \\ T. J. Stratton \\ D. I. Weyns-Rollosson \\ R. A. Hyre \\ C. S. Fein \\ T. W. Hahn \\ K. L. Todd \\ E. J. Vejvoda \\ Los Alamos Technical Associates, Inc. \\ D. R. Duncan \\ Westinghouse Hanford Company \\ Date Published \\ January 1995
}

Prepared for the U.S. Department of Energy Office of Environmental Restoration and

Waste Management

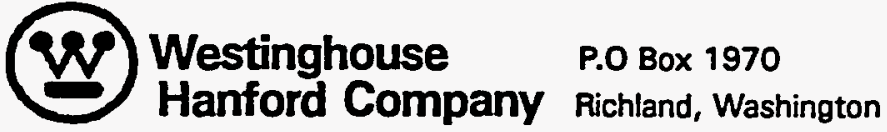

Hanford Operations and Engineering Contractor for the

U.S. Department of Energy under Contract DE-AC06-87RL10930

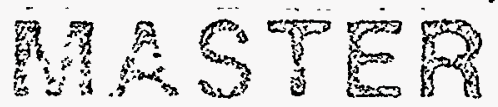

Approved for Public Release 


\section{RELEASE AUTHORIZATION}

\section{Document Number: WHC-EP-0829}

$\begin{array}{ll} & \text { Estimation and Characterization of Decontamination } \\ \text { Document Title: } & \text { and Decommissioning Sol id Waste Expected from the } \\ \text { Plutonium Finishing Plant. }\end{array}$

Release Date: $\quad 1 / 6 / 95$

This document was reviewed following the procedures described in WHC-CM-3-4 and is:

\section{APPROVED FOR PUBLIC RELEASE}

WHC Information Release Administration Specialist:

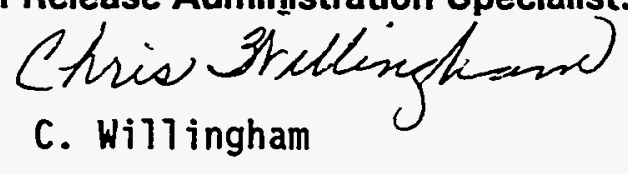




\section{DISCLAIMER}

This report was prepared as an account of work sponsored by an agency of the United States Government. Neither the United States Government nor any agency thereof, nor any of their employees, make any warranty, express or implied, or assumes any legal liability or responsibility for the accuracy, completeness, or usefulness of any information, apparatus, product, or process disclosed, or represents that its use would not infringe privately owned rights. Reference herein to any specific commercial product, process, or service by trade name, trademark, manufacturer, or otherwise does not necessarily constitute or imply its endorsement, recommendation, or favoring by the United States Government or any agency thereof. The views and opinions of authors expressed herein do not necessarily state or reflect those of the United States Government or any agency thereof. 


\section{DISCLAIMER}

Portions of this document may be illegible in electronic image products. Images are produced from the best available original document. 


\title{
ACKNOWLEDGEMENTS
}

The authors wish to recognize the efforts that went into the preparation of this document. The following people were among the key contributors.

Without their valuable expertise and responsive effort, preparation of this document would not have been possible.

\author{
Central Files: $\quad$ Lupe Garza \\ Lois Knapp \\ Lana Perry \\ Record Management: Sylvia Spiel \\ Parren Morrison \\ Technical Drawings: David Spiel \\ PFP Engineers: William (Randy) Buchanan \\ John D. Dick \\ Albert L. Ehlert \\ Micheal B. Nelson \\ Joseph $J$ Roemer \\ Frank F. Walters \\ George A. Westsik
}


WHC-EP-0829

This page intentionally left blank. 


\section{EXECUTIVE SUMMARY}

This study was designed to characterize and estimate the amounts of equipment and other materials that are candidates for removal and subsequent processing in a solid waste facility when the Hanford Site's Plutonium Finishing $\mathrm{Pl}$ ant is decontaminated and decommissioned. The building structure itself and any contaminated soil are not part of this study; it is most probable that they will be dealt with in situ.

The estimates presented are based on available information from documentation, including facility engineering drawings, and from interviews with key PFP personnel. A walkdown was conducted to verify the information gathered from the research.

This information is required to support planning activities for current and future solid waste treatment, storage, and disposal facilities and operations. It also will be included in the Solid Waste Program Technical Baseline Description. Previous estimates of decontamination and decommissioning wastes were quite sketchy and general in nature; little facility-specific detail was available. This report, and others performed for additional 200 Area facilities, are the most accurate and comprehensive to date. The results will be used in waste projection estimates and as part of interface definition between the Solid Waste Division and decontamination and decommissioning generators.

The results of the solid waste characterization indicate that a total of approximately $5,500 \mathrm{~m}^{3}$ of solid waste is expected to result from the 
decontamination and decommissioning of the Plutonium Finishing PTant. The volumes and percentages of waste by category are presented in Table ES-1. Figure ES-1 provides a visual comparison of the relative volumes of solid waste expected. The largest volumes of waste are low-level and transuranic (71 percent and 21 percent, respectively).

Assuming that tanks containing regulated chemicals will be removed from the gloveboxes before decontamination and decommissioning begins, all of the gloveboxes and hoods have been classified as transuranic wastes instead of transuranic mixed wastes. Mixed wastes are mainly from the tanks and pipe work. The large amount of low-level waste $\left(3,913 \mathrm{~m}^{3}\right)$ is mainly from the ventilation systems, which are assumed to be uncompacted. 
Table ES-1. Total Solid Waste Volumes Expected to Result From the Decontamination and Decommissioning of the Plutonium Finishing Plant.

\begin{tabular}{|l|c|c|}
\hline \multicolumn{1}{|c|}{ Waste category } & $\begin{array}{c}\text { Solid waste } \\
\text { expected } \\
\left(\mathrm{m}^{3}\right)\end{array}$ & $\begin{array}{c}\text { Percentage of total } \\
\text { solid waste expected } \\
\left(\mathrm{m}^{3}\right)\end{array}$ \\
\hline Dangerous solid waste & 41 & 1 \\
\hline Low-Tevel waste & 3,913 & 71 \\
\hline Transuranic waste & 1,171 & 21 \\
\hline Transuranic mixed waste & 363 & 7 \\
\hline Total & 5,488 & 100 \\
\hline
\end{tabular}

Figure ES-1. Relative Volumes of Solid Waste Expected to be Generated During the Decontamination and Decommissioning of the Plutonium Finishing Plant.

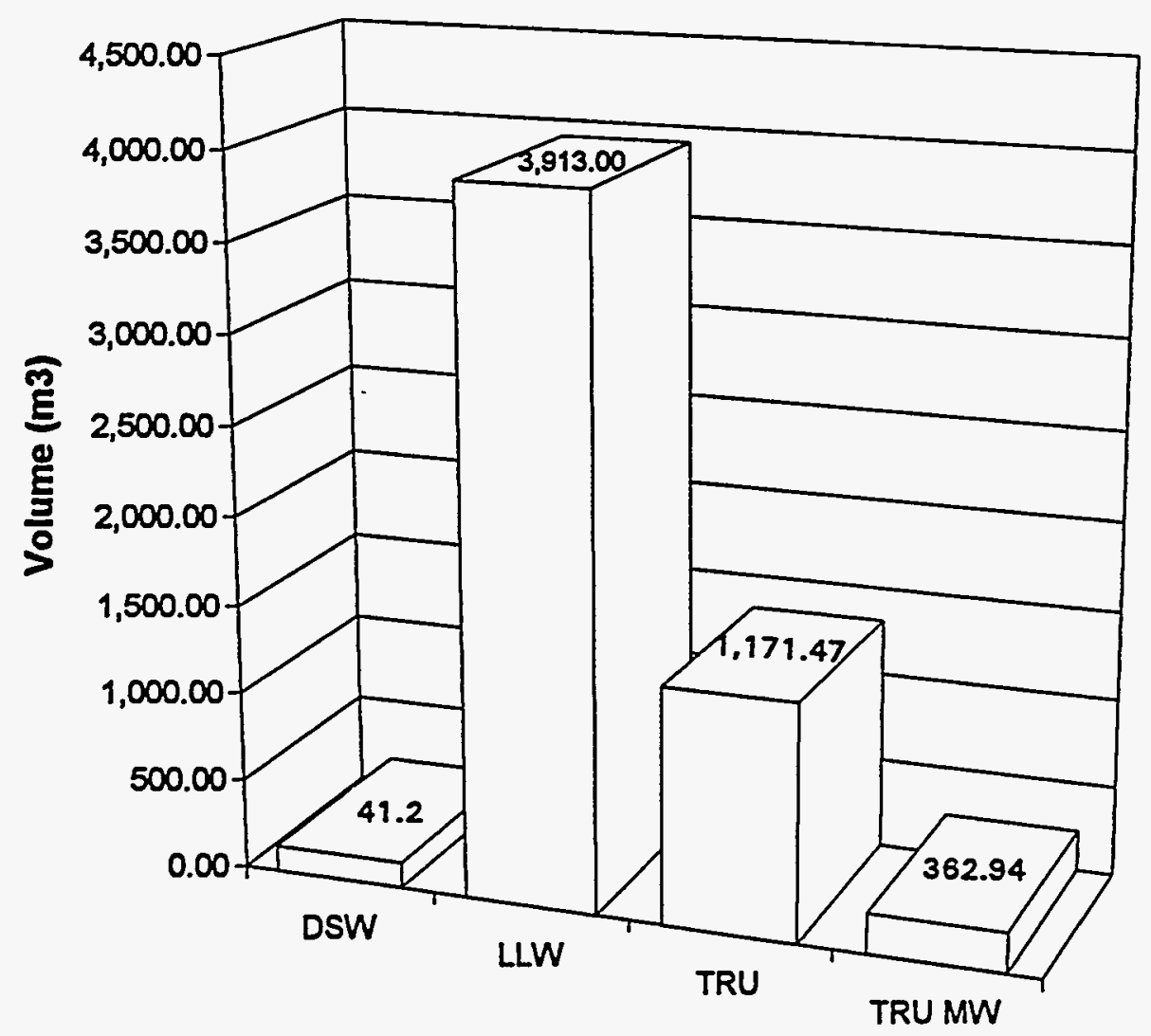


WHC-EP-0829

This page intentionally left blank. 


\section{CONTENTS}

1.0 INTRODUCTION . . . . . . . . . . . . . . . . . . . $1-1$

1.1 PURPOSE ........................ . . . . . . . . . . . . . . . .

1.2 BACKGROUND . . . . . . . . . . . . . . . . . 1-2

1.3 SCOPE . . . . . . . . . . . . . . . . . . $1-4$

2.0 PLANT DESCRIPTION . . . . . . . . . . . . . . . . 2-1

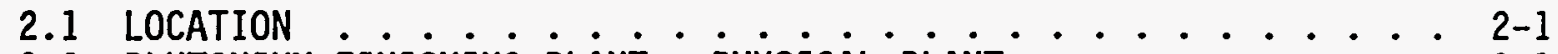

2.2 PLUTONIUM FINISHING PLANT - PHYSICAL PLANT . . . . . . . . . 2-1

2.2.1 234-5Z Building . . . . . . . . . . . . . 2-7

2.2.2 236-Z Building ................. . 2-7

2.2.3 232-Z Building ................. . 2-8

2.2.4 241-Z Building ................ 2-8

2.2.5 242-Z Building ................. . 2-8

2.2 .6 291-Z Building . . . . . . . . . . . . . . 2-9

2.2.7 2736-Z Building ................. 2-9

2.2 .8 2736-ZB Building . . . . . . . . . . . . . . 2-10

2.2.9 243-Z Building ................. 2-10

3.0 PROCESS DESCRIPTION . . . . . . . . . . . . . . . . 3-1

3.1 RECEIVING/SHIPPING . . . . . . . . . . . . . . 3-2

3.2 SORTING . . . . . . . . . . . . . . . . . . 3-4

3.3 STORING . . . . . . . . . . . . . . . . . . 3-5

3.4 REPACKAGING .. . . . . . . . . . . . . . . . 3-5

3.5 WASTE DISPOSAL .................... 3-5

3.6 PLUTONIUM RECOVERY/PURIFICATION . . . . . . . . . . . . . 3-6

3.7 PLUTONIUM CONVERSION . . . . . . . . . . . . . . . 3-7

3.8 PLUTONIUM PROCESS SUPPORT LABORATORY . . . . . . . . . . 3-10

3.9 ANALYTICAL LABORATORY . . . . . . . . . . . . . . . . 3-11

4.0 METHODOLOGY .......................... 4-1

4.1 UNCERTAINTIES ..................... 4-1

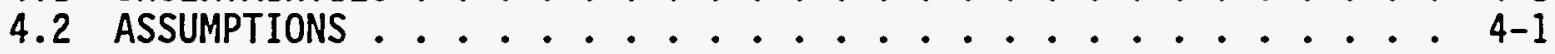

4.3 GENERAL APPROACH .................... . . . . . . . .

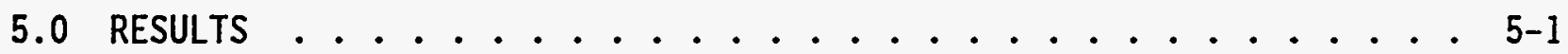

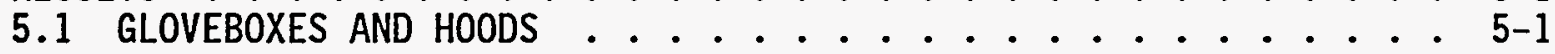

5.2 TANKS CONSIDERED EXTERNAL TO GLOVEBOXES OR HOODS . . . . . . 5-15

5.3 PIPING NOT IN GLOVEBOXES OR HOODS . . . . . . . . . . . 5-16

5.3.1 Waste Drainage Piping .............. . 5-28

5.3.2 234-5Z Service Piping .. . . . . . . . . . . . . 5-31

5.3.3 Plutonium Reclamation Facility Waste Drain

and Service Piping ............... . 5-31

5.4 VENTILATION SYSTEM . . . . . . . . . . . . . . 5-35

5.4.1 234-5Z Supply Systems .............. . 5-43

5.4.2 234-5Z E-3 and E-4 Exhaust . . . . . . . . . . . 5-43

5.4.3 Plutonium Reclamation Facility Supply and Exhaust . . 5-45

5.4.4 241-Z Exhaust ................ . 5-46

5.4 .5 2736-ZA Exhaust ................. 5-46

5.4.6 2736-ZB Supply and Exhaust .............. 5-46

5.4.7 Volume of Ventilation System . . . . . . . . . . 5-46

5.5 MISCELLANEOUS EQUIPMENT .............. . . . . . . . . . . . . 
CONTENTS (cont.)

6.0 SUMMARY ........................... 6-1

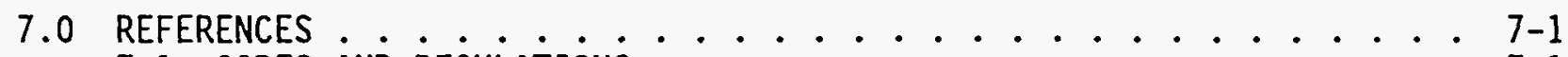

7.1 CODES AND REGULATIONS ................. . . . . . .

7.2 DOCUMENTS . . . . . . . . . . . . . . 7-1

7.3 DRAWINGS .................. . . . . . . .

7.4 U.S. DEPARTMENT OF ENERGY ORDERS .......... $7-5$

8.0 BIBLIOGRAPHY . . . . . . . . . . . . . 8-1 APPENDIXES

A POTENTIAL CONTAMINATION LEVELS AT THE PLUTONiUM Finishing PLANT . . A-I

B ESTIMATION OF PIPING REMOVAL DURING DECONTAMINATION AND

DECOMMISSIONING AT THE PLUTONIUM FINISHING PLANT . . . . . . . . B-1

C ESTIMATION OF VENTILATION REMOVAL DURING DECONTAMINATION AND

DECOMMISSIONING AT THE PLUTONIUM FINISHING PLANT . . . . . . . . C-1 
HHC-EP-0829

LIST OF FIGURES

page

2-1 Location of the Hanford Site ............... 2-2

2-2 Hanford Site Map ................ 2-3

2-3 200 West Area ................. 2-4

2-4 Aerial Photograph of the Plutonium Finishing Plant . . . . . . 2-5

2-5 Main Plutonium Finishing Plant Structures ......... 2-6

3-1 2736-Z Support Facility Solid Fissile Material Handling

Flow Diagram ....................... 3-3

3-2 Load-In/Load-Out Operations Flow Diagram . . . . . . . . . 3-8

3-3 Remote Mechanical C Line Flow Diagram, Nitrate Solution

Through Plutonium Fluoride Production ........... 3-9

4-1 Plutonium Finishing Plant Ventilation Control and

Radiation Control Areas .............. 4-3

4-2 Plutonium Finishing Plant Radiation Dose Levels . . . . . . 4-21

4-3 Location of Radiation Sources in the Plutonium Finishing PIant . . . 4-31

4-4 Location of Gloveboxes Within Buildings 234-5Z and 236-Z . . . . 4-43

4-5 Radiologically Controlled Access Areas in the

Plutonium Finishing Plant ........ . . . . . 4-47

4-6 Radiological Posting as of January 1994 . . . . . . . . . . 4-49

4-7 Building and Room Classification .............. 4-73

5-1 Pipe Plan View Detailing Drainage from 241-Z to the Cribs . . . . . 5-30

6-1 Decontamination and Decommissioning Solid Waste

Classification by Percentage ............. . 6-3

6-2 Decontamination and Decommissioning Solid Waste

Classification by Volume . . . . . . . . . . . . 6-4 
WHC-EP-0829

\section{LIST OF TABLES}

page

4-1 Area Occupancy Factors of Plant Radiation or Potential Radiation Areas ............... . 4-13

4-2 Room Descriptions and Classification . . . . . . . . . . 4-59

5-1 Gloveboxes and Hoods ................... . . 5-3

5-2 Tanks Internal to Gloveboxes . . . . . . . . . . . . . 5-11

5-3 Tanks Considered External to Gloveboxes or Hoods . . . . . . . . 5-17

5-4 Cribs, Ditches, and Trenches Used by PFP in the Past . . . . . 5-25

5-5 Cribs Fed by Overflow from the 241-Z-361 Settling Tank . . . . . 5-26

5-6 Plutonium from Sump Tanks to 241-Z-361 Settling Tank and Cribs . . 5-27

5-7 Drawing List for Pipe Locations . . . . . . . . . . . . . 5-29

5-8 Drain Pipe Volumes . . . . . . . . . . . . . . . . 5-32

5-9 234-5Z Service Pipe Volumes . . . . . . . . . . . . . 5-33

5-10 236-Z Service Pipe Volumes . . . . . . . . . . . . . 5-34

5-11 Volume of High-Efficiency Particulate Air Filters . . . . . . . . 5-36

5-12 Volume of Filterboxes .................. . . 5-40

5-13 Reference Drawing List. . . . . . . . . . . . . . . . . 5-44

5-14 Ventilation System Volume . . . . . . . . . . . . . . 5-44

5-15 Miscellaneous Equipment ................ . 5-47

6-1 Decontamination and Decommissioning Solid Waste Volume Summary . . 6-2 


\section{LIST OF TERMS}

$\begin{array}{ll}\text { CLCS } & \text { closed loop cooling system } \\ \text { D\&D } & \text { decontamination and decommissioning } \\ \text { DOE } & \text { U.S. Department of Energy } \\ \text { DST } & \text { double-shell tank } \\ \text { DSW } & \text { dangerous solid waste } \\ \text { HEPA } & \text { high-efficiency particulate air } \\ \text { LLW } & \text { low-level waste } \\ \text { LLMW } & \text { low-level mixed waste } \\ \text { LLWTF } & \text { Low-Level Waste Treatment Facility } \\ \text { NDA } & \text { nondestructive assay } \\ \text { PCW } & \text { primary closed cooling (loop) } \\ \text { PFP } & \text { Plutonium Finishing Plant } \\ \text { PPSL } & \text { Plutonium Process Support Laboratory } \\ \text { PR } & \text { product removal } \\ \text { PRF } & \text { Plutonium Reclamation Facility } \\ \text { PUREX } & \text { Plutonium-Uranium Extraction (Facility) } \\ \text { RMA } & \text { Remote Mechanical A (Line) } \\ \text { RMC } & \text { Remote Mechanical C (Line) } \\ \text { SCW } & \text { secondary closed cooling (1oop) } \\ \text { SNM } & \text { special nuclear material } \\ \text { TEDF } & \text { Treated Effluent Disposal Facility } \\ \text { TRU } & \text { transuranic } \\ \text { TRU MW } & \text { transuranic mixed waste } \\ \text { TSD } & \text { treatment, storage, and disposal } \\ \text { Z Plant } & \text { Plutonium Finishing Plant } \\ & \end{array}$




\section{LIST OF CONVERSIONS}

Factors for Unit Conversions.

\begin{tabular}{|c|c|}
\hline Quantity & ४ै. Equivalent values $\%$ \\
\hline Mass & $\begin{array}{l}1 \mathrm{~kg}=1,000 \mathrm{~g}=0.001 \text { metric ton }=2.20462 \mathrm{lb}=35.27392 \mathrm{oz} \\
1 \mathrm{lb}_{\mathrm{m}}=16 \mathrm{oz}=5 \times 10^{-4} \text { ton }=453.593 \mathrm{~g}=0.453593 \mathrm{~kg}\end{array}$ \\
\hline Length & $\begin{aligned} 1 \mathrm{~m} & =100 \mathrm{~cm}=1,000 \mathrm{~mm}=10^{6} \text { microns }(\mu \mathrm{m})=10^{10} \text { angstroms }(\AA) \\
& =39.37 \text { in. }=3.2808 \mathrm{ft}=1.0936 \mathrm{yd}=0.0006214 \mathrm{mile} \\
1 \mathrm{ft} & =12 \text { in. }=1 / 3 \mathrm{yd}=0.3048 \mathrm{~m}=30.48 \mathrm{~cm}\end{aligned}$ \\
\hline Volume & $\begin{aligned} 1 \mathrm{~m}^{3} & =1,000 \text { liters }=10^{6} \mathrm{~cm}^{3}=10^{6} \mathrm{ml}=35.3145 \mathrm{ft}^{3} \\
& =220.83 \text { imperial gallons }=264.17 \mathrm{gal}^{3}=1056.68 \mathrm{qt} \\
1 \mathrm{ft}^{3} & =1728 \text { in. }=7.4805 \mathrm{gal}=0.028317 \mathrm{~m}^{3}=28,317 \mathrm{~L} \\
& =28.317 \mathrm{~cm}^{3}\end{aligned}$ \\
\hline Force & $\begin{array}{l}1 \mathrm{~N}=1 \mathrm{~kg} \cdot \mathrm{m} / \mathrm{s}^{2}=10^{5} \text { dynes }=10^{5} \mathrm{~g} \cdot \mathrm{cm} / \mathrm{s}^{2}=0.224811 \mathrm{~b}_{\mathrm{f}} \\
1 \mathrm{lb}_{\mathrm{f}}=32.174 \mathrm{lb} \mathrm{b}_{\mathrm{m}} \cdot \mathrm{ft} / \mathrm{s}^{2}=4.4482 \mathrm{~N}=4.4482 \times 10^{5} \text { dynes }\end{array}$ \\
\hline Pressure & 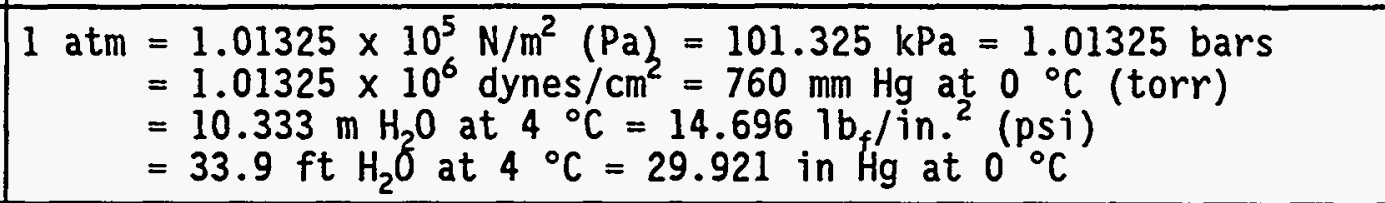 \\
\hline Energy & $\begin{array}{l}=1 \mathrm{~N} \cdot \mathrm{m}=10^{7} \text { ergs }=10^{7} \text { dyne } \cdot \mathrm{cm}=2.778 \times 10^{-7} \cdot \mathrm{Kw} \cdot \mathrm{h} \\
=0.23901 \mathrm{cal}=0.7376 \mathrm{ft} \cdot 1 \mathrm{~b}_{\mathrm{f}}=9.486 \times 10^{-4} \mathrm{Btu}\end{array}$ \\
\hline Power & $\begin{array}{l}=1 \mathrm{~J} / \mathrm{s}=0.23901 \mathrm{cal} / \mathrm{s}=0.7376 \mathrm{ft} \cdot 1 \mathrm{~b}_{\mathrm{f}} / \mathrm{s}=9.486 \times 10^{-4} \\
\text { Btu } / \mathrm{s} \\
=1.341 \times 10^{-3} \mathrm{hp}\end{array}$ \\
\hline
\end{tabular}

Example: The factor to convert grams to $7 b_{m}$ is $\left[\frac{2.20462 \mathrm{Ib}_{\mathrm{m}}}{1000 \mathrm{~g}}\right]$. 
WHC-EP-0829

\section{ESTIMATION AND CHARACTERIZATION OF DECONTAMINATION \\ AND DECONMISSIONING SOLID WASTE EXPECTED \\ FRON THE PLUTONIUM FINISHING PLANT}

\subsection{INTRODUCTION}

\subsection{PURPOSE}

The purpose of this report is to characterize and estimate the amount of solid waste that will be generated during the decontamination and decommissioning (D\&D) of the Hanford Site's Plutonium Finishing Plant (PFP). This characterization is to include estimates of waste volume, dose rate, hazardous constituents, radionuclide content, and dimensions of bulky equipment or structures for the following classes of waste (Pottmeyer et a1. 1994).

- Transuranic Waste - Without regard to source or form, transuranic (TRU) waste is waste that is contaminated with alpha-emitting transuranium radionuclides with half-lives greater than 20 years and concentrations greater than $100 \mathrm{nCi} / \mathrm{g}$ of waste matrix. Transuranium radionuclides are radionuclides having an atomic number greater than 92. In addition to TRU radionuclides, radium sources and ${ }^{233} U$ in concentrations greater than $100 \mathrm{nC} i / g$ of waste matrix are managed as TRU waste.

- Transuranic Mixed Waste - TRU mixed waste (TRU MW) is waste that meets the definition above for TRU waste and contains dangerous waste in addition to the radioactive components. Dangerous wastes are defined in the Washington Administrative Code (WAC 173-303-040).

- Low-Level Waste - Low-level waste (LLW), as defined in U.S. Department of Energy (DOE) Orders 5820.2A and 5400.3, is radioactive waste not defined as high-level waste, TRU waste, spent nuclear fuel, or byproduct material. All LLW is also classified according to Category 1,3 , and greater than Category 3 concentration limits. These limits are based on a similar waste classification system developed by the U.S. Nuclear Regulatory Commission in 10 CFR 61 and are explained in more detail in the Hanford Site Solid Waste Acceptance Criteria (Will is 1993).

- Low-Level Mixed Waste - Low-level mixed waste (LLMW) is LLW that contains dangerous waste as defined in WAC 173-303-040.

- Nonradioactive Dangerous Solid Waste - Any nonradioactive sol id waste that has been contaminated by hazardous chemicals as defined in WAC 173-303-040 is regulated as dangerous solid waste (DSW).

The waste volume and characterization information gathered in this study will support planning activities for current and future solid waste treatment, storage, and disposal (TSD) facilities and operations. 


\subsection{BACKGROUND}

The PFP, located in the 200 West Area within DOE's Hanford Site in southcentral Washington State, was designed prior to 1946 and completed in 1949. Additional process facilities were completed between 1949 and 1992 . The facility consists of eight main buildings and associated support buildings. These buildings include the following:

- PFP, 234-5Z

- Plutonium Reclamation Facility (PRF), 236-Z

- Liquid Waste Disposal Facility, Building 241-Z

- Ventilation Exhaust Facility, Building 291-Z

- Plutonium Storage Facility, 2736-Z Complex

- Waste Treatment Facility, 242-Z (americium recovery)

- Incinerator, Building 232-Z

- Low-Level Waste Treatment Facility (LLWTF), 243-Z.

The principal PFP structure, Building 234-5Z, was completed in 1949 to purify plutonium nitrate solutions, reduce the nitrate to plutonium metal, and fabricate plutonium metal parts. The 234-5Z Building houses plutonium processing operations, the Analytical Laboratory (Engineering Laboratory), the Plutonium Process Support Laboratory (Development Laboratory), and the major service and support facilities. The 241-Z Liquid Waste Disposal Facility and the 291-Z Building, which houses ventilation exhaust fans and mechanical service equipment, were built at the same time as the 234-5Z Building. A metal building was constructed over the 241-Z sump tanks in 1978.

The original design of the PFP complex provided for locating all of the planned operations and laboratories within the 234-5Z Building except for waste collection and disposal. However, with increases in production, storage, and scrap recovery requirements, four major additions were made. The Waste Incinerator Facility, Building 232-Z, was designed to recover plutonium from combustible scrap and was completed in 1961. The PRF, Building 236-Z, and the 242-Z Waste Treatment Facility were completed in 1964. The Plutonium Storage Facility, 2736-Z, and the 2736-ZA Building, which houses ventilation exhaust fans and mechanical service equipment, were built in 1971 . The Product Shipping and Receiving Facility, 2736-ZB, and the 2721-Z Building, which houses backup diesel generators, were built in 1979 and 1982 , respectively. Recently, the LLWTF and the Closed Loop Cooling System (CLCS) were completed as a part of the Hanford Site's waste minimization program.

The PFP was used to conduct diversified plutonium processing, storage, and support operations. Operations conducted at the PFP included the following:

- Processing of plutonium-containing materials from which it was economical to recover plutonium and convert it to specification nitrate and metal forms

- Storing, and preparing for shipping, special nuclear materials (SNM), primarily plutonium

- Performing development work for processes involving SNM 
- Purifying plutonium nitrate solutions to meet specifications for conversion to solid form (PRF)

- Converting purified plutonium nitrate solutions to a plutonium metal or plutonium oxide (Remote Mechanical A [RMA] and Remote Mechanical C [RMC] Lines)

- Fabricating plutonium metal (function transferred to Rocky Flats during the 1970 's)

- Managing all of the plutonium inventory at Hanford, including receipt, storage, and shipment.

The PRF processed material with economically recoverable quantities of plutonium to provide purified plutonium nitrate solutions; the RMA and RMC Lines converted the solutions to metal buttons; the 2736-Z complex received, stored, packaged, and shipped plutonium solids (including oxide powder and button products); and the 234-5Z Building 1aboratories provided the space and equipment to perform development work.

A7though production processes at the PFP have shut down, plutoniumbearing materials remain in the plant. Chemically reactive materials expose workers to radiation and present an unnecessary risk to the public. The DOE has recently decided that these plutonium-bearing materials may be treated on a case by case basis as waste if that would reduce worker exposure and public risk. In response to public concerns, DOE has ordered an Environmental Impact Statement that will cover remediation activities and the actions necessary to make the facility ready for D\&D. In addition, DOE plans to take immediate actions to improve the conditions that are safety concerns for PFP workers.

The Pacific Northwest Laboratory is currently studying ways to stabilize the various plutonium-bearing materials in both the PFP process and vault storage areas. Until materials are moved out of the PFP process areas, the plant cannot be cleaned out and deactivated.

Current activities at the PFP, driven by safety concerns, are detailed below. These actions were selected by DOE because they do not produce a pure product, nor do they release carbon tetrachloride to the air or discharge liquids to the ground. They also generate smaller amounts of liquid waste, relative to other projects considered, to be sent to Hanford's double-sheli tanks (DSTs) (WHC 1994b).

- Stabilize sludge in gloveboxes - Much of the plutonium-bearing sludge stored in PFP gloveboxes can be heated and converted to an impure stable solid that can be stored safeiy in PFP's vaults for many years. Because storage of the resulting material doesn't require constant handling, having the material stabilized reduces radiation exposure to workers. An environmental assessment is being prepared to review potential environmental impacts from this operation. This process would use two small new laboratory furnaces, in glovebox $\mathrm{HC}-21 \mathrm{C}$, to heat the sludge to about $500{ }^{\circ} \mathrm{C}$ and then to $1000^{\circ} \mathrm{C}\left(900^{\circ} \mathrm{F}\right.$ to $\left.1800^{\circ} \mathrm{F}\right)$ over several hours. This process converts the plutonium compounds to plutonium oxide, drives off the moisture, and results in a stable oxide powder. Other 
chemicals not driven off by the heating process will remain as stable solids. This stabilization process has been operated in the past at the PFP using older furnace designs.

- Eliminate the risk of leaks of chemical solutions stored in "10-L" containers - Some storage containers, known as "10-Ls," holding plutonium-bearing chloride solutions need to be repackaged or to have the solutions stabilized. The plastic bottles holding the solutions inside these containers are expected to be brittle from exposure to radiation and are at risk of cracking in the future. These solutions will be put in safe, new storage containers. Some or all of the solutions will be used in the Plutonium Process Support Laboratory to test future processing options to support the environmental impact statement. The chloride solutions were not considered for the PRF stabilization run because they would have damaged the processing equipment.

- Download solutions from the PRF to eliminate the risk of tank leaks - Chemical solutions used for training PRF facility operators are no longer needed. The nitrate solutions, which are low in plutonium content, have been transferred to the Hanford Site's DSTs. Organic solutions were drained from the tanks, put in glass bottles with absorbent pads, packaged in steel drums, and disposed of as solid waste. This effort is complete.

- Remove duct work and clean up surface contamination - Portions of the PFP's ventilation duct work and piping that add to radiation exposure risk will be dismantled and cleaned out to remove plutonium holdup. This ducting and piping is no longer used in the plant. Areas of surface contamination will be cleaned to reduce the risk of worker contamination. Removed materials will be packaged as solid waste.

- Reduce plutonium inventory in the $232-z$ Building - The removal of unnecessary ducting and pipe from 232-Z will likewise be conducted. This building is not seismically qualified and presents the greatest risk of releasing radioactivity in case of an earthquake at Hanford. Cleanout of the ducts will remove the bulk of the plutonium and will prepare the building for final D\&D.

\subsection{SCOPE}

The major sections of this document and the topics they cover are outlined briefly below.

Section 2.0 provides a physical description of the PFP emphasizing the potentially contaminated areas related to the processes performed at the facility throughout its history.

Section 3.0 describes the PFP processes performed over the facility's history and refers to contaminated process areas within the plant. It also helps to explain the plant layout and how the various processes fit together. 
Section 4.0 explains the methodology used to estimate the solid waste types and volumes expected to be processed by solid waste TSD facilities as a result of D\&D activities. The uncertainties and assumptions implicit in the estimation methodology are also presented.

Section 5.0 presents the results of the study. Volumes and waste types are given by natural physical category (i.e., gloveboxes and hoods, tanks, pipes, duct work, and miscellaneous equipment).

Section 6.0 summarizes the solid waste volumes for the facility by waste type (i.e., DSW, LLW, LLMW, TRU, and TRU MW).

Section 7.0 lists the references used during the project.

Appendix $A$ includes the data used to classify the PFP Buildings and rooms. This classification was then used to focus the project on the contaminated areas of the facility. The data are presented in a table for reference.

Appendix $B$ includes the data and spreadsheet calculations used to estimate the amount of piping that is likely to be removed during the D\&D of the PFP.

Appendix $C$ includes the data and spreadsheet calculations used to estimate the amount of duct work (and other ventilation items) that is likely to be removed during the D\&D efforts. 


\section{WHC-EP-0829}

This page intentionally left blank. 


\subsection{PLANT DESCRIPTION}

The PFP, more familiarly known as $Z$ Plant, is a collection of facilities that primarily has been used for the production of plutonium and plutoniumcontaining materials to support national defense activities. The SNM that formed the feed for PFP processing included, but was not limited to, plutonium-bearing caps, incinerator ash, silicon nitride crucibles, polystyrene cubes, plutonium-uranium mixtures, slag and crucible fragments, unirradiated fuel rods, plutonium-beryllium sources, and unique plutoniumbearing solutions (e.g., plutonium nitrate solution from the Plutonium-Uranium Extraction [PUREX] Facility).

The PFP used separate process lines to handle each of these unique feed streams, resulting in a number of rooms and buildings within the plant dedicated to a particular process. Varying demand by the defense industry, as the war and post-war eras progressed, resulted in numerous changes in the processes that took place at the PFP as well as in the types of equipment required to support these processes. The purpose of this section is to describe the main process building and the process support buildings that comprise the facility. A detailed process history of the plant is provided in the following section with references to these buildings so that solid waste volume estimates from each area can be compared to the generating process to determine the expected contamination.

\subsection{LOCATION}

The PFP is located within DOE's Hanford Site in south-central Washington State (Figure 2-1). It is situated in the west-central region of the Hanford Site within the 200 West Area (Figure 2-2). The 1ocation of the PFP in relation to the other facilities in the 200 West Area is shown in Figure 2-3. An aerial photograph of the PFP facility can be found in Figure 2-4.

\subsection{PLUTONIUM FINISHING PLANT - PHYSICAL PLANT}

The buildings that comprise the PFP are shown in Figure 2-5. The primary process and support buildings include 232-Z, 234-5Z, 236-Z, 241-Z, 242-Z, $291-Z, 2736-Z$, and 2736-ZB. A physical description of each of these buildings follows. As some processes within the PFP Buildings are no longer operational, the status of each of the above mentioned buildings is provided based on the following criteria.

- Active - An active process is currently operating or is scheduled for future operation.

- Standby - A standby process is not currentiy operating, but is operable with the appropriate repairs or upgrades. Facilities on standby have no future operations currently scheduled.

- Layaway - A Tayaway process is not operable without major repairs or upgrades, and no operations are planned. This category is scheduled for terminal cleanout operations/D\&D. 
Figure 2-1. Location of the Hanford Site.

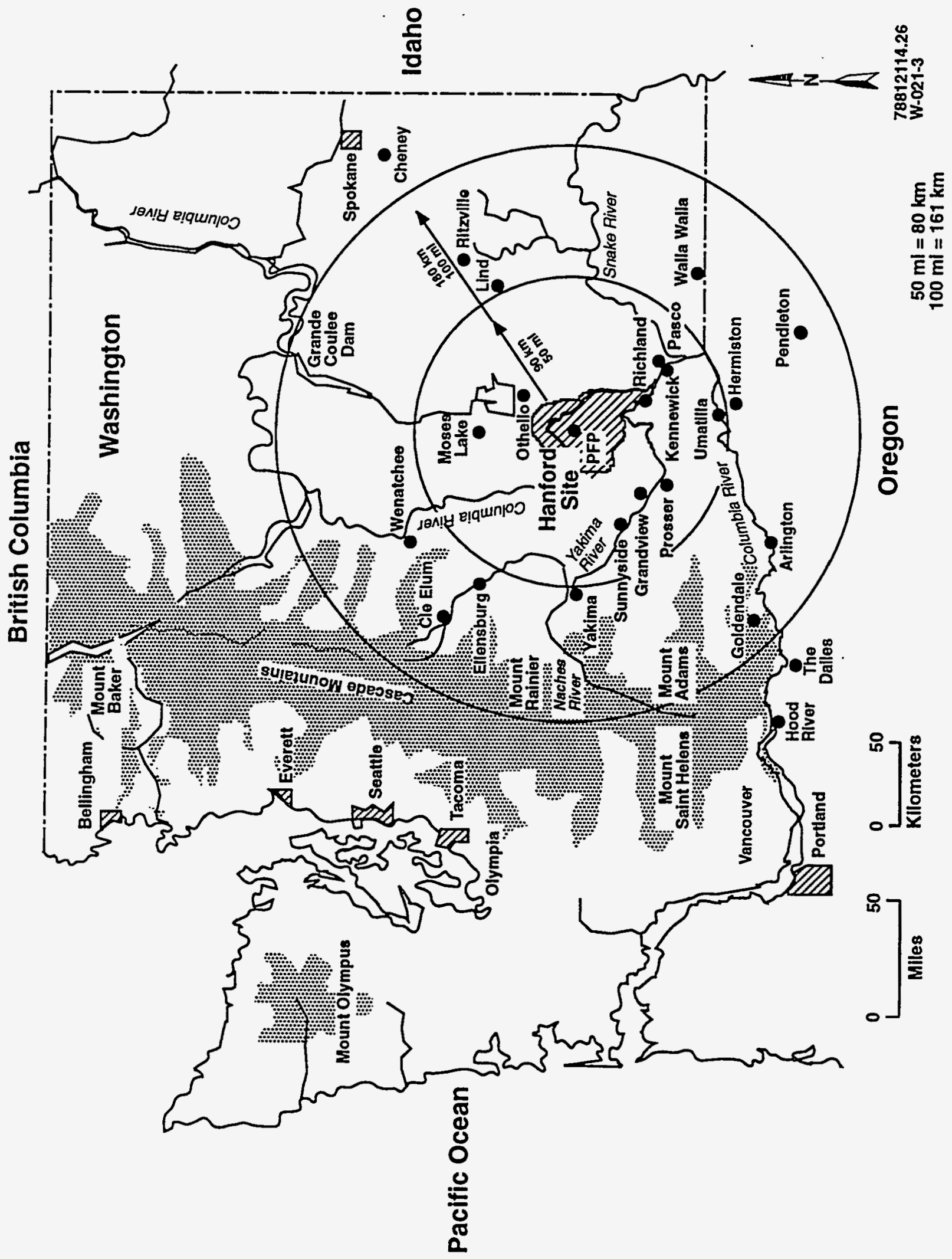


HHC-EP-0829

Figure 2-2. Hanford Site Map.

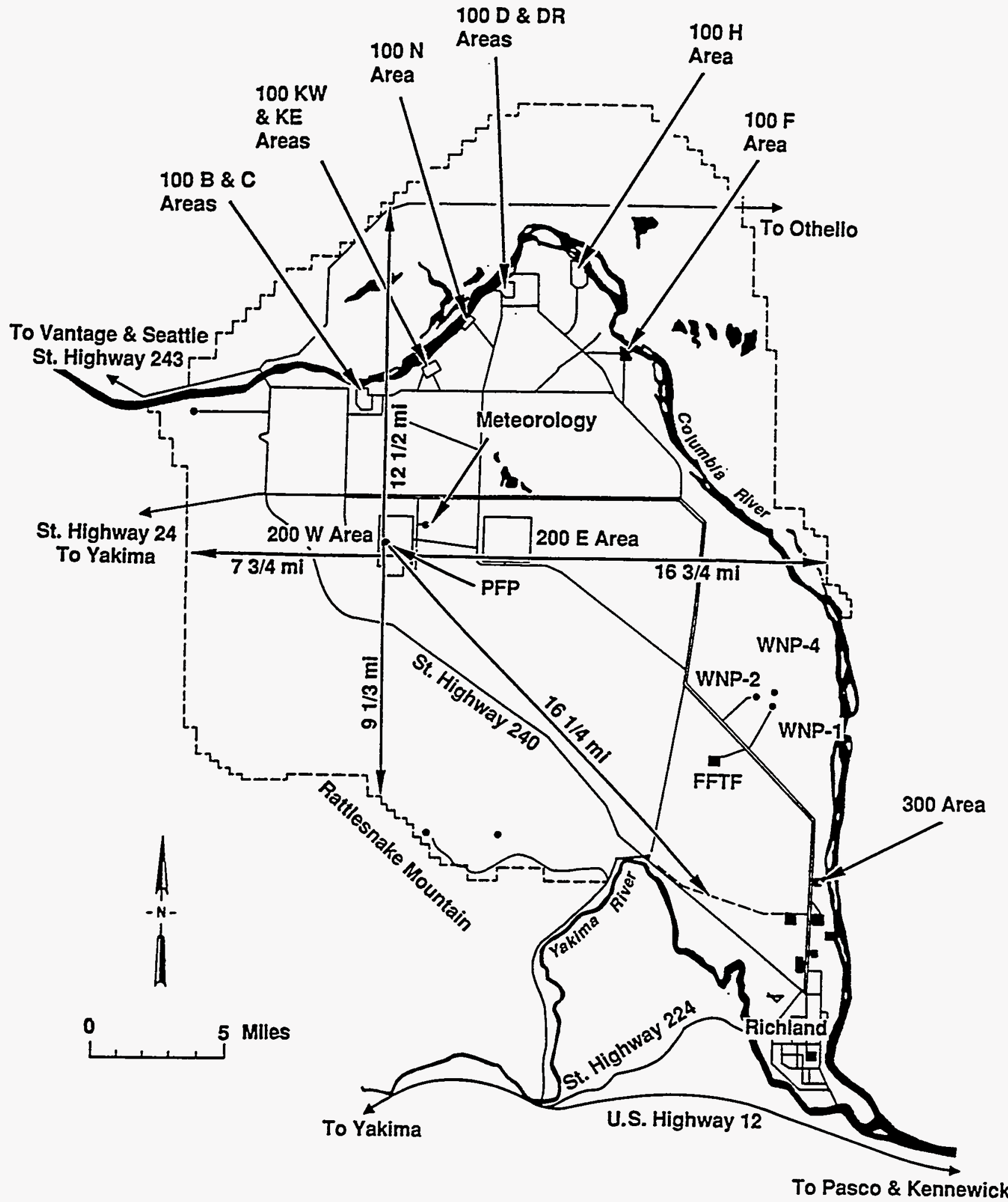

78810007.118 
WHC-EP-0829

Figure 2-3. 200 West Area.

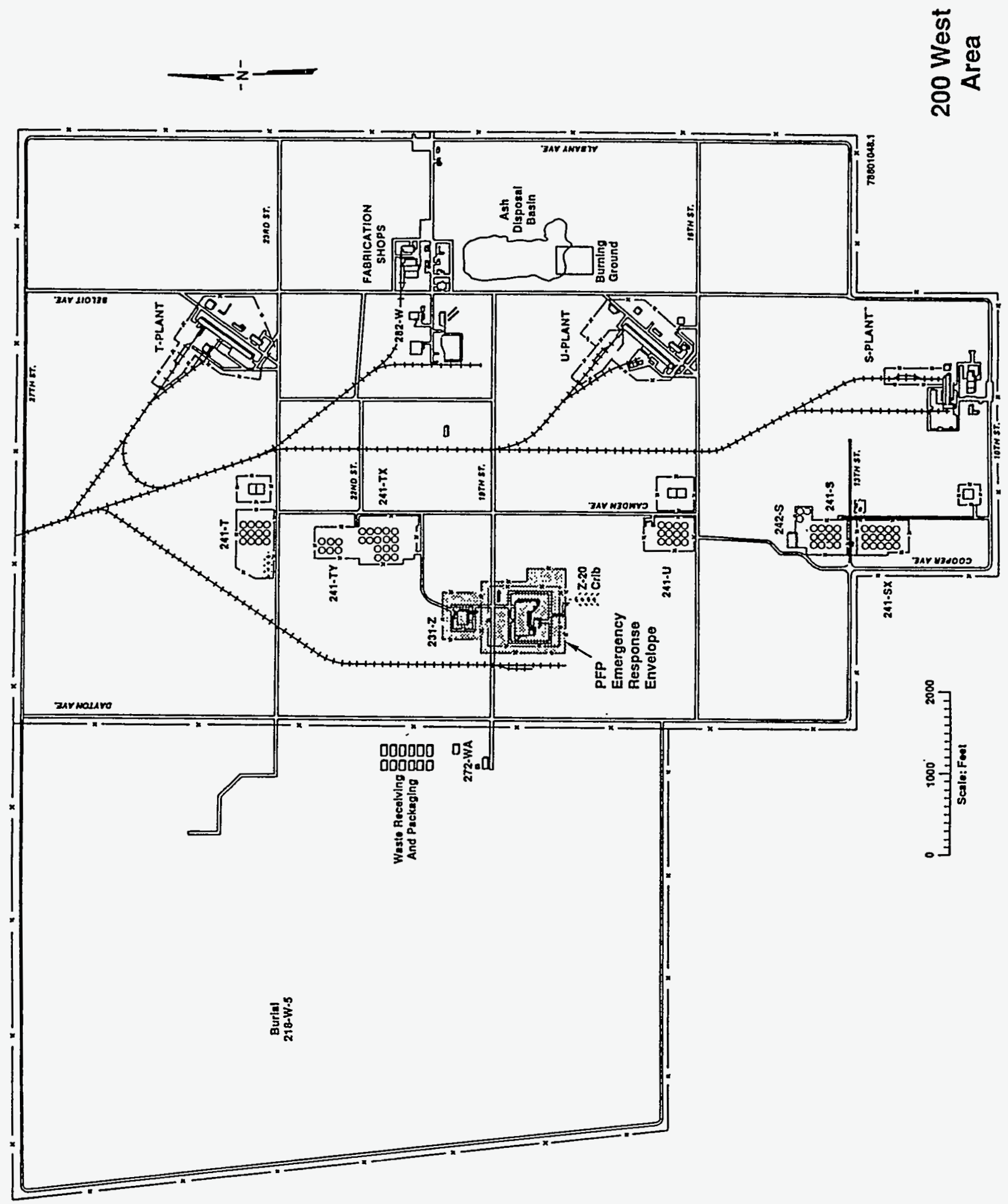


Figure 2-4. Aerial Photograph of the Plutonium Finishing Plant.

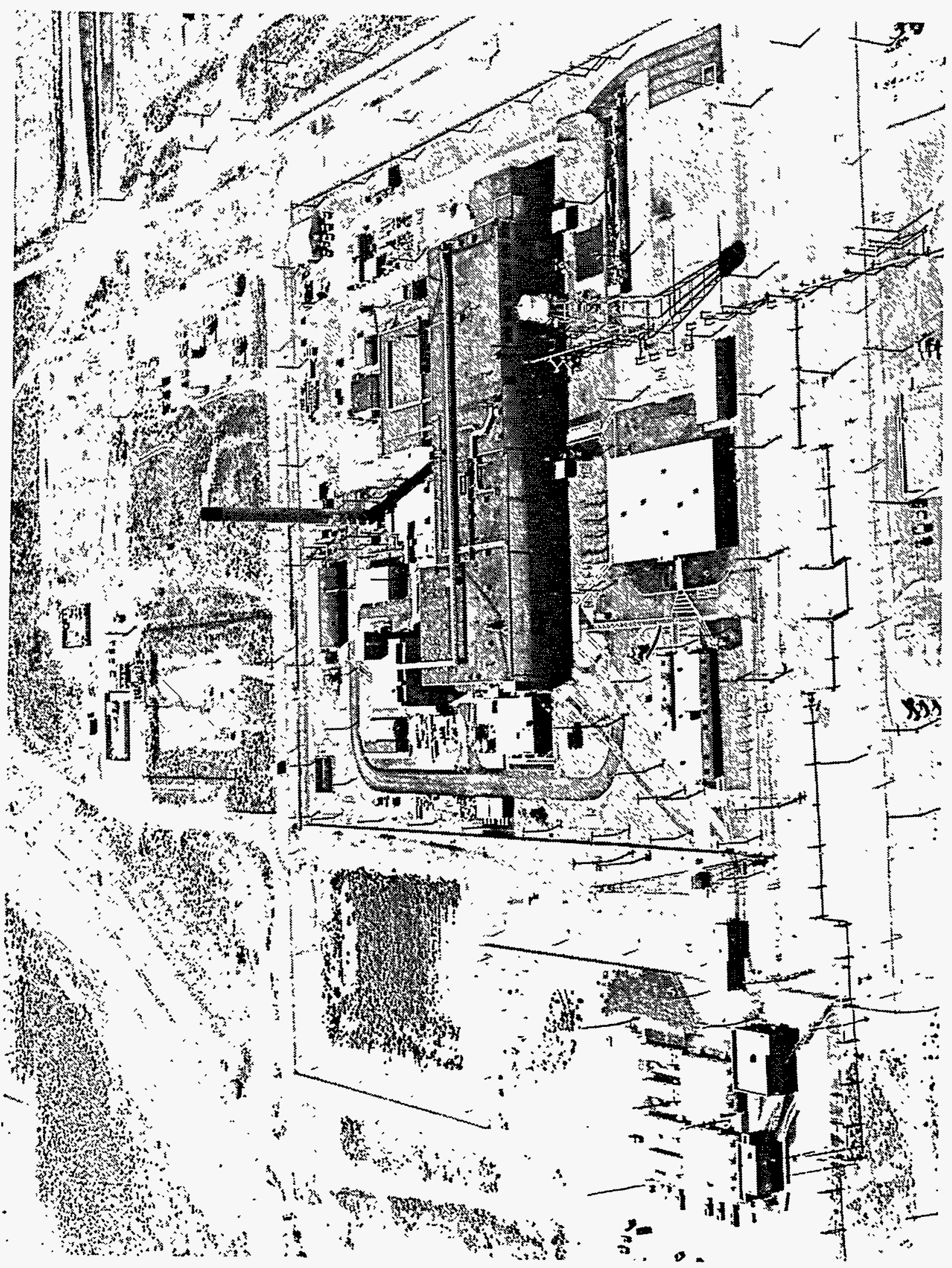


Figure 2-5. Main Plutonium Finishing Plant Structures.

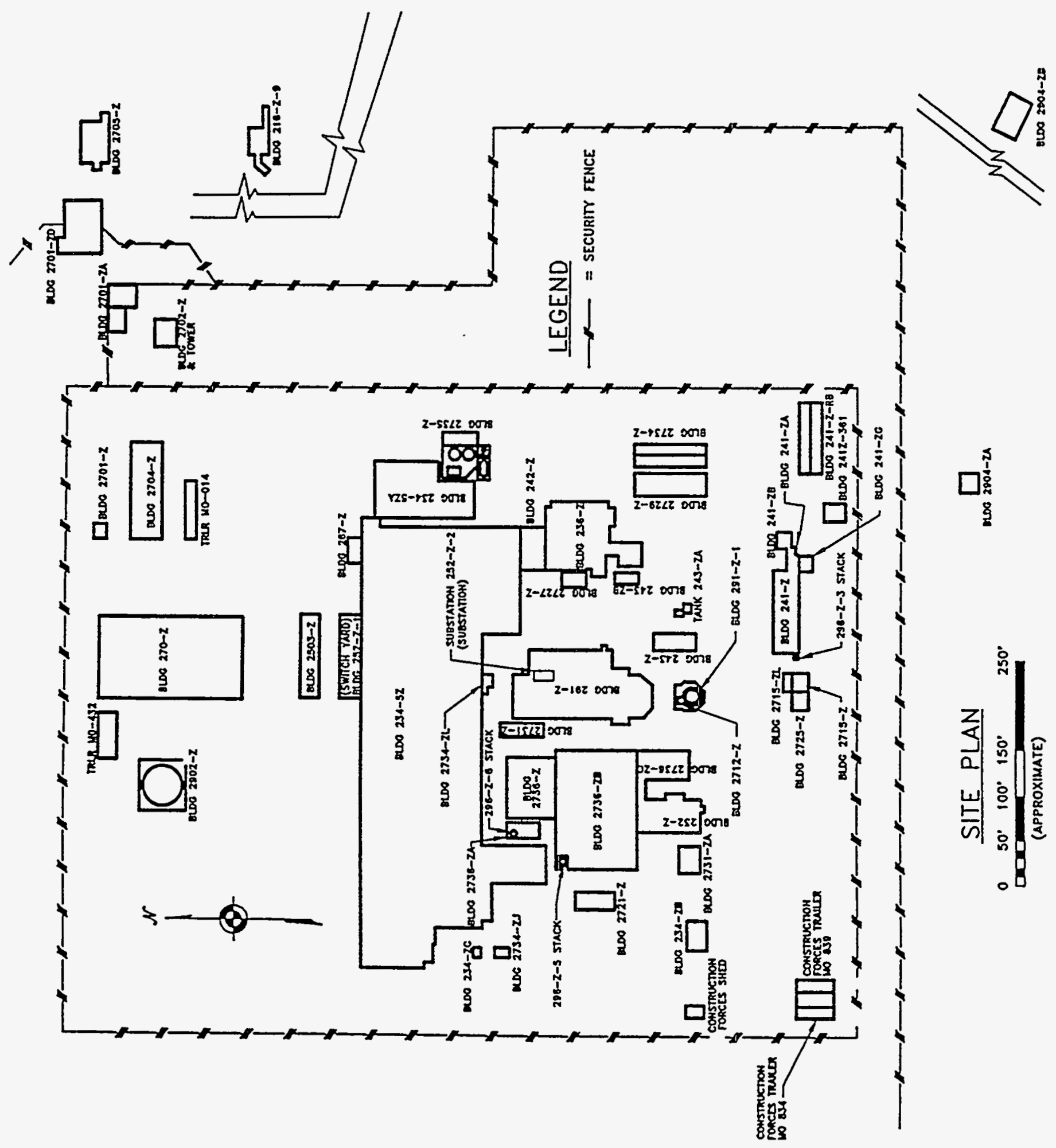


WHC-EP-0829

\subsubsection{4-5Z Building (Active)}

The 234-5Z Building is approximately $55 \mathrm{~m}$ (180 ft) wide by $152 \mathrm{~m}$ (500 ft) long and extends from $3 \mathrm{~m}(9.5 \mathrm{ft})$ below grade to $8 \mathrm{~m}(26.8 \mathrm{ft})$ above grade. Floor levels are designated as tunnel, first floor, duct level, second floor, and roof level.

The 234-5Z tunnel mostly consists of pipe tunnels carrying drain piping to the 241-Z sumps. The first floor houses the two plutonium processing 7 ines (RMA and RMC Lines) and their control rooms; scrap stabilization gloveboxes; plutonium storage vaults; the plutonium nitrate feed load-in/load-out, blending, and storage facilities; the Analytical Laboratory; the Plutonium Process Support Laboratory; the instrument maintenance shops; and rest rooms and office spaces. The duct level contains most of the service piping, ventilation ducts, and some filterboxes. The lunchroom, conference room, materials storage room, chemical feed preparation and aqueous makeup rooms, locker rooms with change facilities and rest rooms, and office spaces are on the second floor. Also on the second floor are exhaust air duct works, including filterboxes and filter rooms. The fan room, located on the northwest corner of the second floor, houses the ventilation supply fans, the steam inlet and distribution system, air dryers, the distilled water still, air chilling units (now inactive), and the power control room.

\subsubsection{6-Z Building (Standby)}

The 236-Z Building is located at the southeast corner of the 234-5Z Building and is connected to it by the 242-Z Building (see Figure 2-5). The 236-Z Building, built as the CAC-880 Project, houses the PRF. It is also referred to as 880, PRF, Plutonium Nitrate Production Facility, and 236.

The building is essentially a four-story structure surmounted by a two-story penthouse. It dimensions are about $24 \mathrm{~m}(79 \mathrm{ft})$ wide by $22 \mathrm{~m}$ $(71 \mathrm{ft})$ long. Its outstanding internal structural feature is a single process equipment cell that is $10 \mathrm{~m}$ (32 ft) wide by $16 \mathrm{~m}(52 \mathrm{ft})$ long.

Maintenance shop facilities are located on the service (east) side of the building on the ground floor. The second floor of the service side is used for a maintenance glovebox and ventilation exhaust filters. Building service equipment and electrical switch gear are on the third floor of the service area. The fourth floor was used for chemical preparation and miscellaneous treatment, and contains the operating control room, slag and crucible dissolver equipment, and a column room in which vertical sections of two liquid-liquid extraction columns, penetrating the room from above and below, are housed in a glovebox. The first four floors are served by a service elevator located within the east side of the building.

The process cell has a $0.6-\mathrm{m}-(2-\mathrm{ft}-)$ thick concrete wall between the cel1 and the "access" hoods. These access hoods are stainless steel paneled hoods containing glass viewing windows and Hypalon hood gloves. Located on both sides of the cell on the first two floors, the hoods contain process

1 Hypalon is a trademark of E. I. du Pont de Nemours \& Company. 
WHC-EP-0829

piping, pumps, valves, flow meters, and other equipment that requires frequent maintenance. The cell floor is covered with a stainless steel liner extending $46 \mathrm{~cm}$ (18 in.) up the side wall. The remaining cell wall and ceiling surfaces are covered with chemical-resistant coatings. Water-filled viewing windows on the third floor have adjacent remote control stations for the cell crane.

\subsubsection{2-Z Building (Layaway)}

The 232-Z Building houses the layaway Contaminated Waste Recovery Process, also known as the "incinerator." It was constructed by Project CGC-013, Plutonium Recovery Process, and was partially decontaminated and decommissioned in 1984.

The 232- $Z$ Building is located about $61 \mathrm{~m}(200 \mathrm{ft})$ south of the main portion of the 234-5Z Building and about $31 \mathrm{~m}$ (100 ft) west of the 291-Z stack. Its approximate dimensions are $11 \mathrm{~m}(37 \mathrm{ft})$ wide by $17 \mathrm{~m}(57 \mathrm{ft})$ long. It is divided into areas for process, storage, change room, chemical preparation, ventilation, and electrical equipment. Except for ventilation supply and exhaust filtration, it uses services from the 234-5Z Building. The 232-Z Building exhaust ventilation system has been isolated from the $291-Z$ Building although it was connected in the past.

Currently, the $232-Z$ Building is being cleaned out to remove unnecessary contaminated ducts and pipes in order to reduce the risk of radiation exposure. Additionally, areas of surface contamination are being cleaned to reduce the risk of worker contamination. Cleanout will remove the bulk of the plutonium holdup and prepare the building for final D\&D (WHC 1994b).

\subsubsection{1-Z Building (Active)}

The 241-Z Building is designated as the Waste Treatment Facility. It is commoniy called the $241-Z$ sumps and in the past was called the $216-Z$ large waste sump tanks. It is a buried structure, with a sheet metal enclosure over the top that houses a hoist for removing cell covers. It consists of five separate enclosures, or ventilated cells, each containing a tank, with a capacity of $15,900 \mathrm{~L}(4,200 \mathrm{ga} 7)$, used to accumulate the liquid wastes generated in the PFP before transfer to the tank farms. Built of reinforced concrete, its approximate dimensions are $6 \mathrm{~m}(20 \mathrm{ft})$ wide by $28 \mathrm{~m}(92 \mathrm{ft})$ long.

At the southwest corner of the $241-z$ vault deck is the equipment for the $241-Z$ vessel vent and vault ventilation system. The $7-m-(24-\mathrm{ft}-)$ high stack, 296-Z-3, and its associated fans, filters, and controls are located on a $4.3-\mathrm{m}$ $(14-\mathrm{ft})$ by $5.5-\mathrm{m}(18-\mathrm{ft})$ concrete pad.

\subsubsection{2-Z Building (Layaway)}

The 242-Z Building houses portions of the Waste Treatment and Americium Facility, which are in layaway and planned for future D\&D. Built primarily by Project CGC-912, this building is referred to as 912 or WT. 
The 242-Z structure is between the southeast corner of the 234-5Z Building and the 236-Z Building. Along its east side runs a corridor connecting the 234-5Z, 242-Z, and 236-Z Buildings. At its west end is an entrance enclosure for outside entry into both $242-Z$ and the Analytical Laboratory in the 234-5Z Building.

The building's approximate dimensions are $12 \mathrm{~m}(40 \mathrm{ft})$ wide, $8 \mathrm{~m}$ (26 ft) long, and $7 \mathrm{~m}(23 \mathrm{ft})$ high. The south portion, approximately $12 \mathrm{~m}(40 \mathrm{ft})$ wide by $3 \mathrm{~m}(10 \mathrm{ft}$ ) long, is the tank room (tank cell). This room extends the full inside building height. The north portion, designated the control room, has a mezzanine over its west half for chemical addition tanks.

The tank room in the 242-Z Building houses large process vessels, which are piped to the process gloveboxes of the control room. The 242-Z Building shares the main ventilation system in common with the 234-5Z and 236-Z Buildings and is equipped with the other PFP utilities and services.

The function of the $242-Z$ facility was to treat waste streams from the PRF for americium recovery and further plutonium reclamation. On August 30, 1976, there was an explosion of the W-14A column in glovebox WT-2 caused by the introduction of concentrated nitric acid onto a degraded cation exchange resin loaded with americium. This explosion grossly contaminated the facility. The process was terminated and the gross contamination cleaned up. Remaining contamination was stabilized and isolated to some extent. Entries were made into the 242-Z process area/control room on December 11, 1984, and into the 242-Z tank room on February 1, 1985, to establish current contamination levels, dose rates, facility configuration, and facility conditions. It was found that contamination was leaching through the fixative in the process area/control room and was loose and widespread throughout the tank room. The dose rate around glovebox WT-2 ranged from 100 to $350 \mathrm{mrem} / \mathrm{h}$ but was significantly less in the remainder of the facility. The general condition and cleanliness of the facility was poor, especially in the tank room, where a slimy, "organic" layer covered a majority of the floor (Duncan 1985).

\subsubsection{1-Z Building (Active)}

The 291-Z Building, also known as the Exhaust Fan House, Exhaust Air Stack Building, and Compressor and Fan House, is a reinforced-concrete structure located approximately $16 \mathrm{~m}(53 \mathrm{ft})$ south of the central part of the 234-5Z Building. Of irregular shape, its approximate dimensions are $23 \mathrm{~m}$ (74 ft) wide by $44 \mathrm{~m}$ (143 ft) long. Its overall height is approximately $7 \mathrm{~m}$ (23 ft) with only $1.2 \mathrm{~m}(4 \mathrm{ft}$ ) above grade. This building houses the exhaust fans, the mechanical service equipment, and the substation. Auxiliary to the 291-Z Building is the 61-m- (200-ft-) high 291-Z-1 stack.

\subsubsection{6-Z Building (Active)}

Building $2736-Z$ is the primary PFP Plutonium Storage Facility. The $2736-Z$ Building is approximately $20 \mathrm{~m}$ (65 ft) long by $17 \mathrm{~m}$ (56 ft) wide. It is constructed of reinforced-concrete wal1 s, $36 \mathrm{~cm}$ (14 in) thick, supported by a cast-in-place concrete slab, $17 \mathrm{~cm}(6.5 \mathrm{in})$ thick. The building consists of 
four rooms for the storage of SNM and is divided by a corridor running the width of the building. Each storage room is approximately $8.5 \mathrm{~m}$ (28 ft) by $8.5 \mathrm{~m}(28 \mathrm{ft})$ in size. Rooms 1,3 , and 4 contain storage cubicles, while room 2 has steel shelves and open floor storage.

\subsubsection{6-ZB Building (Active)}

Building 2736-ZB, located immediately south of $2736-Z$, is approximately $40 \mathrm{~m}(132 \mathrm{ft})$ by $27 \mathrm{~m}(90 \mathrm{ft})$ with reinforced-concrete walls (except for administrative areas) and roof.

The 2736-ZB shipping and receiving areas each provide approximately $93 \mathrm{~m}^{2}$ $\left(1,000 \mathrm{ft}^{2}\right)$ of floor space to accommodate a maximum of 100 shipping containers the size of 208-L (55-gal) drums. Containers are spaced to meet Westinghouse Hanford Company's criticality prevention and personnel exposure specifications as well as to allow corridor access to staging areas. The two areas are physically separated by a wall. The majority of shipping containers handled contain $\mathrm{PuO}_{2}$ powder, plutonium metal, or miscellaneous solid scrap materials from various onsite and offsite sources.

\subsubsection{3-Z Building (Active)}

In May 1994 the LLWTF, located in the 243-Z Building, began receiving very low activity waste from various parts of the PFP via manhole 4 , treating it, and discharging it to manhole 6 . The purpose of the LLWTF is to lower the effluent very low activity waste concentration so that it can be sent to the 200 Area Treated Effluent Disposal Facility (TEDF). The TEDF will be accepting all processed very low activity waste from the PFP on or before September 30, 1995. Until the liquid TEDF comes on-line, liquid effluent is being discharged to the $216-\mathrm{Z}-20 \mathrm{crib}$.

The average daily flow rates through the facility have varied from 19.7 to $75.7 \mathrm{~L} / \mathrm{min}(5.2$ to $20 \mathrm{gal} / \mathrm{min})$ depending upon the weather conditions with $113.6 \mathrm{~L} / \mathrm{min}$ (30 gal/min) being the maximum set single train flow rate. 


\subsection{PROCESS DESCRIPTION}

The PFP, or Z Plant, began operations in late 1949 to process plutonium and prepare plutonium products. Before 1949 all plutonium nitrate solutions had been shipped to other onsite facilities or offsite for further processing.

Plutonium was brought into the PFP as a liquid nitrate solution. The plutonium was precipitated as the oxalate, converted to the fluoride, and reacted at high temperature with metallic calcium, forming the metal. The slag and crucible were routed to a plutonium recovery process; in later years, the buttons were remelted and cast into the finished shape. Certain finishing operations were performed: the shape was coated with nickel and polished, enabling it to be handled without spreading plutonium contamination. Up to this point, all of the plutonium operations were conducted in tightly sealed enclosures (gloveboxes and hoods).

At startup, the finishing operations were very labor intensive. After 1953, much of the process was automated to reduce worker exposure. P7utonium recovery operations started in 1955 at the Recuplex facility (234-5Z, room 221).

The following is a timetable of the key operations performed at the PFP (Ballinger and $\mathrm{Hall} 1991$ ):

1949 Start-up of plutonium finishing in 234-5Z Building

1953 Original process automated to reduce worker radiation exposure

1955 Startup of Recuplex (234-5Z, room 221); recovery of plutonium from finishing plant liquid waste

1961 Startup of incinerator and leach facilities (232-Z) to recover plutonium from solid waste

1962 Criticality accident in Recuplex; facility shutdown

1964 Startup of PRF to replace Recuplex

1965 Americium recovery starts in $242-Z$

1971 Startup of plutonium oxide blending and production in 234-5Z

1973 Incinerator and leach facilities shutdown

1976 Americium ion exchange explosion; americium recovery shutdown; 234-5Z shutdown

1978 Restart of $234-5 Z$ operations

1979 High stack release; PRF shutdown; plutonium metal and oxide production ends

1984 PRF started up 


\section{PRF shutdown}

1987 Plutonium metal line started up

1989 The PFP production processes shutdown.

Processing at the PFP handled three types of feed materials in order to produce plutonium metal. Each of the feed types was handled differently, depending on the process lines. The purpose of initial feed processing was to prepare a purified plutonium nitrate solution that could be processed through a solvent extraction step and subsequently reduced to product. Processing at the PFP also included fabrication of plutonium metal into useful parts.

Liquid waste from the PFP contained only minor amounts of fission products. However, it did contain low concentrations of plutonium and other TRU elements and was high in metallic nitrates. Initially, this waste was discharged via cribs to soil columns, which sorbed the TRU elements and kept them close to the point of discharge. Later, waste from the PFP was stored in underground tanks (Ballinger and Hall 1991).

The PFP process had seven main components. They were

- Receiving/shipping

- Sorting

- Storing

- Repackaging

- Waste disposal

- Plutonium recovery/purification

- Plutonium conversion.

In addition, the PFP facility supported the Plutonium Process Support Laboratory and the Analytical Laboratory. The purpose of this section is to describe the processes that took place in the areas and laboratories described above, as well as to relate these processes and the areas they took place in to the buildings that were discussed in the previous section on the physical plant.

\subsection{RECEIVING/SHIPPING}

Solid plutonium material was received at the $2736-\mathrm{ZC}$ loading dock, and nitrate solutions were received at the west loading dock $(234-Z C)$ of the 234-5Z Building. The containers were brought into Building 2736-ZB for inspection, weighing, and nondestructive assay (NDA) measurement of fissile material contents. If the materials were determined to be unstable (e.g., gas evolution, liquid), a recovery plan was developed and implemented that provided interim safe storage and stabilization. Stable, solid materials were moved to 2736-Z for storage until final disposition. See Figure 3-1 for a complete description of the 2736-Z support facility's solid fissile material handling. For a physical description of the 234-5Z, 2736-Z, and 2736-ZB Buildings, see Section 2.0, "Physical Plant." 


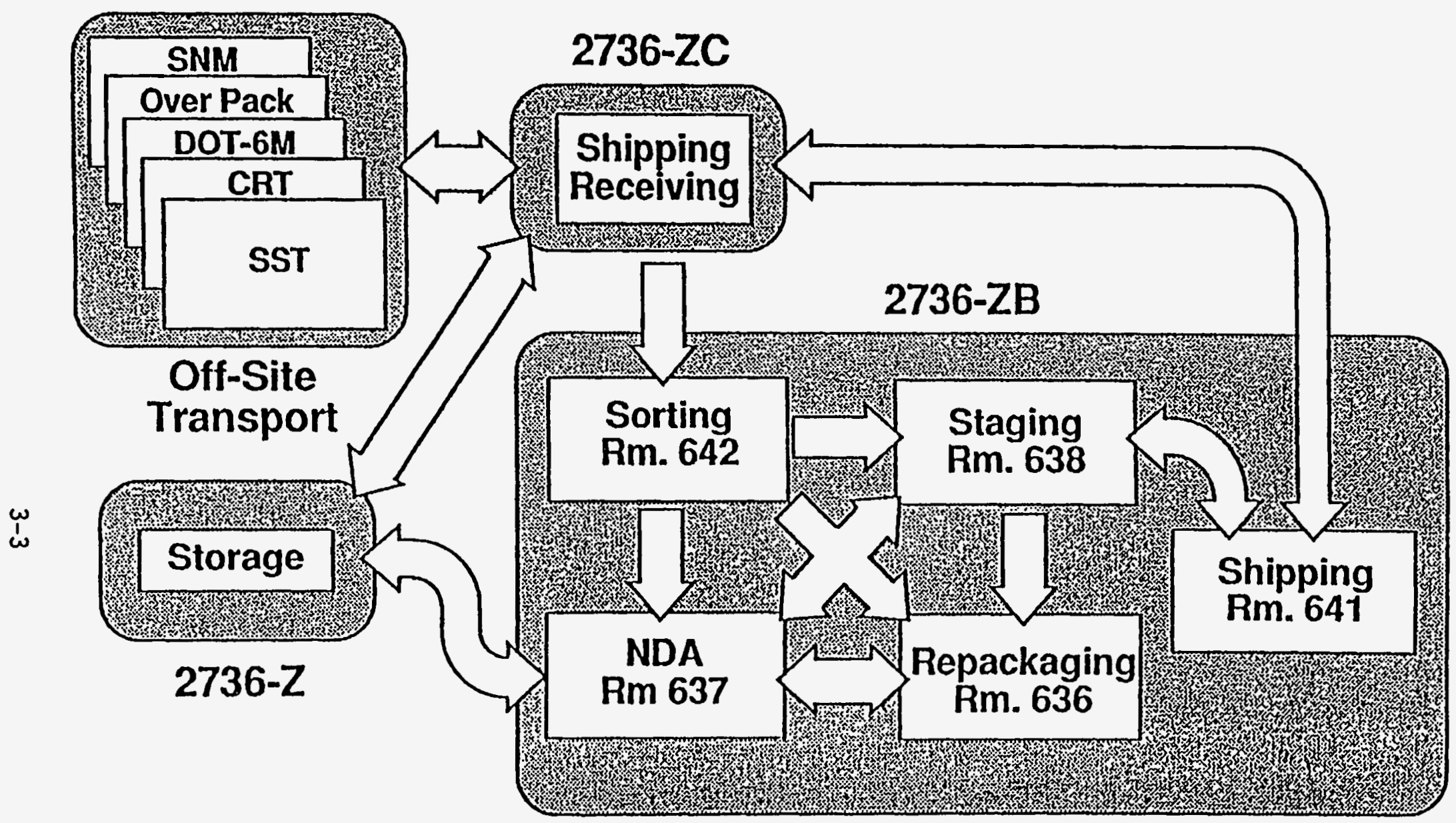


Nitrate solutions, such as $\mathrm{Pu}\left(\mathrm{NO}_{3}\right)_{4}$, were shipped and received from the 2734-ZC dock. Load-in/load-out was accomplished in room 227 of

Building 234-5Z in glovebox HC-227S and hood HC-227T. Glovebox HC-227S was used to blend and sample plutonium nitrate and to transfer solutions to the PRF or to nitrate staging tanks in glovebox HC-4. Room 227 was a dual-purpose facility that supported both the RMC Line and plutonium recovery in the PRF. The solutions were sampled and analyzed for plutonium as well as contaminants. The amount of SNM received was calculated from volume and analytical data. The solutions normally were sent to the RMC Line, although they may have been sent to PRF for purification and/or concentration.

Product removal (PR) containers, marked "Mark IV/V, Emergency," were transported to 234-5Z, room 236, and vault 192-A for storage.

Fuel-grade $\mathrm{PuO}_{2}$ from PUREX was also received at 2736-ZC and stored in Building 2736-Z before shipment to the Savannah River Plant or other DOE Sites. The oxide could be repackaged in $2736-Z B$ if necessary.

Only solid, stable, plutonium-bearing materials were shipped offsite. The final products of the RMC Line were referred to as metal buttons. The cans containing metal buttons, after they had been held in storage for an extended period of time, were transferred to room 636, Building 2736-ZB, and moved into the repackaging glovebox. Each plutonium metal button was removed from the package, inspected, and if loose oxide was present, it was removed by brushing with a paint brush. The button was weighed, recanned, and bagged out into a food pack can.

The packages to be shipped were placed in shipping containers and moved to the 2736-ZC dock where they were placed in cargo restraint transporters that were secured in trucks for shipment.

\subsection{SORTING}

Material containers were transferred to room 642, 2736-ZB Building, for sorting. Sorted containers were transferred to staging room 638 where they were prepared for repackaging, shipping, or storage in Building 2736-Z. Staging area activities included inspection and weighing; pneumatic, hydrostatic, and leak testing of shipping containers when necessary; and decontamination of empty shipping containers.

Plutonium-bearing materials that were known to be reactive were moved to an appropriate glovebox in 234-5Z Building. The material was confined within a ventilated control barrier until it could be processed to a stable form.

Plutonium bearing-material with insufficient characterization to confirm that it was stable was defined as potentially unstable. It was inspected in gloveboxes in 2736-ZB, and the source data were reviewed to determine whether it should be handled as reactive or stable material. 
WHC-EP-0829

\subsection{STORING}

Extraordinary precautions were taken in the handling and storage of SNM because of its unique properties. Varying quantities of plutonium metal, $\mathrm{PuO}_{2}$, plutonium compounds, recoverable plutonium-bearing material, salts, and unirradiated reactor fuel were stored in vault and "vault-type" rooms at the 234-5Z, 2736-Z, and 2736-ZB Buildings. Also, drums containing TRU waste were temporarily stored. The areas for storage of SNM were:

\begin{tabular}{|c|c|c|c|}
\hline \multicolumn{2}{|c|}{$234-5 Z$} & $2736-$ Z & $2736-$ ZB \\
\hline Room 174 & Room 192-B & Vault 1 & Room 637 \\
\hline Room 175 & Room 192-C & Vault 2 & Room 638 \\
\hline Room 190 & Room 225 & Vault 3 & Room 641 \\
\hline Room 192-A & Room 236 & Vault 4 & Room 642 \\
\hline
\end{tabular}

The stable/shippable materials, such as plutonium metal and $\mathrm{PuO}_{2}$, were stored in vaults 1 through 3 in the 2736-Z Building.

\subsection{REPACKAGING}

Containers that either failed in storage or had been damaged in transit were repackaged in Building 2736-ZB, room 636. The source containers, type $3-L$, were opened, and using remote handling devices, the sources were withdrawn and verified. Repackaging also occurred in Building 234-5Z, rooms 235-C and 230-A. Glovebox $\mathrm{HC}-21-\mathrm{C}$ in room 230-A was used to repackage $\mathrm{PuO}_{2}$ and plutonium-bearing materials. Glovebox 235-B2 in room 235-C was routinely used to repackage large containers (i.e., 208-L [55-gal] drums) that did not fit into the other gloveboxes. Glovebox 235-B2 was also used to dismantle and repackage high-efficiency particulate air (HEPA) filters.

\subsection{WASTE DISPOSAL}

Combustible glovebox waste was sealed out of gloveboxes, bagged, and loaded in 22.7-kg (50-1b) 1ard cans. These containers measure $30.48 \mathrm{~cm}$ (12 in.) in diameter by $38.1 \mathrm{~cm}$ (15 in.) high and have a volume of $0.02 \mathrm{~m}^{3}$ $\left(0.71 \mathrm{ft}^{3}\right.$ ) (WHC 1993). The cans were then transported to room 637 for NDA measurement by the NaI package counter. Once a plutonium value was assigned to the waste package, it was placed in a 208-L (55-gal) waste drum for interim storage. When the drum was ful1, or had reached allowable 1 imits for plutonium or hydrocarbon materiais, it was sealed and stored pending transport to TRU waste storage and disposal.

Room waste, as generated, was held in waste drums in several rooms throughout the PFP. These drums were intended for the disposal of non-TRU waste and only low-level plutonium contamination was allowed in them. When a drum was full, it was sealed and transported to room 637 for NDA and staged in room 638 for disposal. 
Rags were used, particularly in PRF, for clean-up operations. The rags generaliy had a considerable amount of plutonium nitrate and acid on them. Rinsing them removed the plutonium nitrate and neutralized the acid. A rag rinsing station was provided in the 200 East Area glovebox in sections EL and EM.

Noncombustible wastes were placed in specially designated waste drums, one for glovebox waste and another for room waste.

\subsection{PLUTONIUM RECOVERY/PURIFICATION}

The plutonium recovery process converted various plutonium-bearing materials and aqueous feeds to a purified plutonium nitrate product suitable for conversion to plutonium metal or $\mathrm{PuO}_{2}$. Most of the processes were located in the PRF in the 236-Z Building. Some of the miscellaneous processing took place within 234-5Z Building of the PFP. Sections 2.2.1 and 2.2.2 provide a physical description of Buildings $234-5 Z$ and $236-Z$, respectively.

Most of the operating equipment in the PRF cell was controlled from the central control room, room 44, on the fourth floor of the 236-Z Building.

Recovery of plutonium in aqueous solution, from plutonium-scrap materials $\left(\mathrm{PuO}_{2}\right.$ powders, incinerator ashes, sludge, and miscellaneous solutions) that were suitable for the solvent extraction process, took place in gloveboxes in the PRF and in the 234-5Z Buildings. Several processes, known as miscellaneous treatment, were contained within a complex of five gloveboxes in room 41 of the PRF. The gloveboxes in room 41 are numbered one through six (glovebox MT-2 has been removed). The first glovebox was used for inspection and batch dissolver charge mixing. Glovebox 3 was used to dissolve bulk metallic plutonium in $\mathrm{HNO}_{3}$ using electrolytic dissolvers. Glovebox 4 could be used for residue stabilization and recovery of $\mathrm{PuO}_{2}$ by distilling polystyrene cubes and burning residual carbon. Glovebox 5 was used for dissolution and leaching of plutonium-leachable solids. Glovebox 6 was used for clarifying dissolver solution and cementing the solid residues (after NDA) for waste disposal; it also contained the vacuum system used for transferring solution. In addition, glovebox HA-23S in the 234-5Z Building was used for storage of reactive scrap.

Two nearly identical continuous dissolver/condensers, used as slag and crucible dissolvers, are installed in the 236-Z Building canyon. Each dissolver is equipped with an acid feed system and an offgas treatment system. However, either dissolver may be valved to any of the acid feed or offgas treatment systems. Both dissolvers may be operated simultaneously if desired.

Feed solutions were transferred into the feed storage tanks from a number of sources including miscellaneous treatment, aqueous waste processing, and aqueous plutonium nitrate scrap loaded into 234-5Z g7ovebox 227-S from PR cans. In feed preparation, chemicals were added to the plutonium aqueous solution to produce a feed solution suitable for processing by solvent extraction.

The plutonium extraction and stripping function took place in two columns, the CA column and the CC column. The lower section of each column is 
in the 236-Z process cell, and the upper sections extend up through gloveboxes on the fourth, fifth, and sixth floor penthouse of the building. These columns were generaliy used for extraction of plutonium from a feed solution of plutonium nitrate. However, the two columns could be used for the coextraction of plutonium and uranium from a mixed plutonium-uranium feed.

Product concentration was designed to increase the plutonium content in the aqueous product from the solvent extraction CC column from a nominal value of $50 \mathrm{~g} / \mathrm{L}$ to about $300 \mathrm{~g} / \mathrm{L}$. To remove trace amounts of tributyl phosphate, the plutonium nitrate solution first flowed to a tank for deentrainment. In this tank the aqueous solution passed through $\mathrm{CCl}_{4}$ to remove any residual organic entrained in the solution.

There are two gloveboxes located in the 234-5Z Building that were used for scrap stabilization. Glovebox $\mathrm{HC}-60$, located in room $230 \mathrm{C}$, was used for hydrolysis of scrap containing high organic concentrations. Glovebox HA-40F, located in room 169, was used for calcining ash from the incinerator at the Rocky Flats Plant in Colorado.

Glovebox HC-46F, Tocated in room 170 of Building 234-5Z, was designed for inspection, sampling, screening, dissolution clarification, product transfer, and waste handling of plutonium-bearing solids containing less than 20 percent organic material. It contains equipment for dissolving plutonium-bearing solids. Glovebox HA-21I, in room 235B, contains furnaces that also could be used for calcining hydrolyzed scrap.

Several NDA systems were available for package evaluation. The NDA Laboratory is located in room 637, Building 2736-ZB.

\subsection{PLUTONIUM CONVERSION}

The RMC Line conversion of plutonium nitrate to plutonium metal started with a lot of nitrate solution selected from various sources, such as the PUREX Facility, the PRF, and stored PR cans, to find the right mix of plutonium isotopes. The lot was loaded into the glovebox HC-227S bank tanks via the HA-227T load-in facility (see Figure 3-2).

The solutions were blended by circulating through the HC-227S bank tanks. Samples were taken and analyzed to ensure that the desired blend was obtained. The blending could also be done in tanks in glovebox $\mathrm{HC}-4$, which served as a staging glovebox for feed to the RMC Line after the solutions were transferred from HC-227S. For a complete overview of the RMC process see Figure 3-3.

The final product of the RMC process was a metal button. After the material was processed, the button was picked up with tongs and placed in the chute leading to glovebox HC-17SBB where it was pickled in acid. After pickling approximately 15 buttons, the acid became saturated with slag particles. The solution was then transferred to the aqueous catch tank in glovebox HC-7. Eventually it was transferred to the PRF for plutonium recovery. After 24 hours, the button was again moved out, via conveyor $\mathrm{HC}-2$, to glovebox $\mathrm{HC}-21 \mathrm{C}$, where it was canned. 
Figure 3-2. Load-In/Load-Out Operations Flow Diagram.

\section{$\underline{\mathrm{RMC}}$}

Load In Concentrated Plutonium Nitrate Solutions

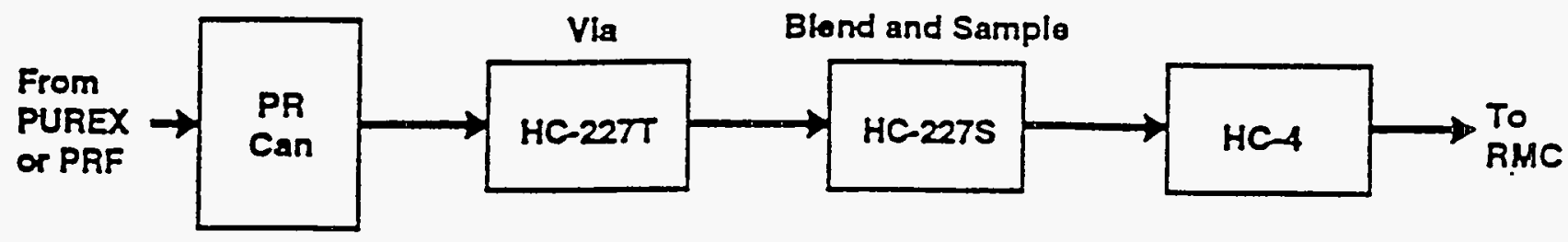

\section{Load Out Dilute Solutions}

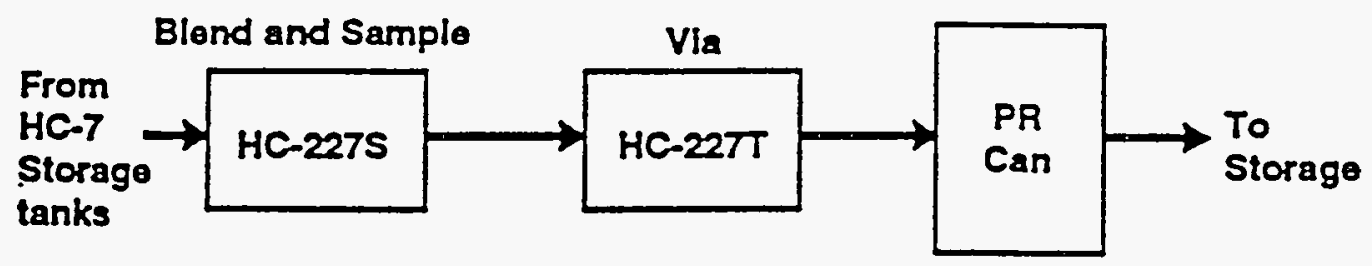

\section{PRF}

Load In Concentrated Filtrate or Other Dilute Plutonium Nitrate Solutions

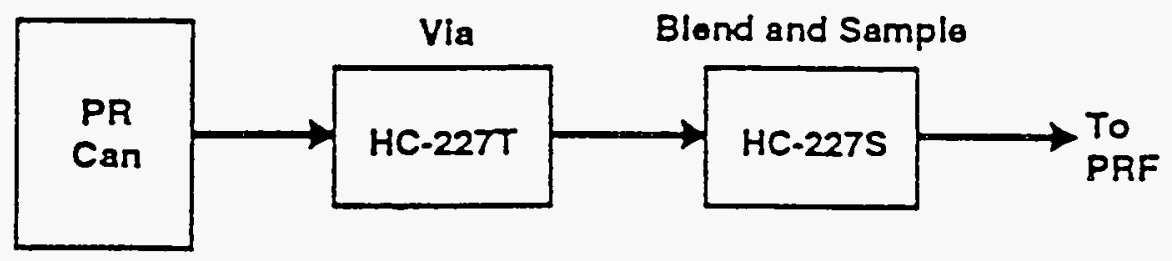

Load Out Concentrated Plutonium Nitrate or Filtrate Solutions

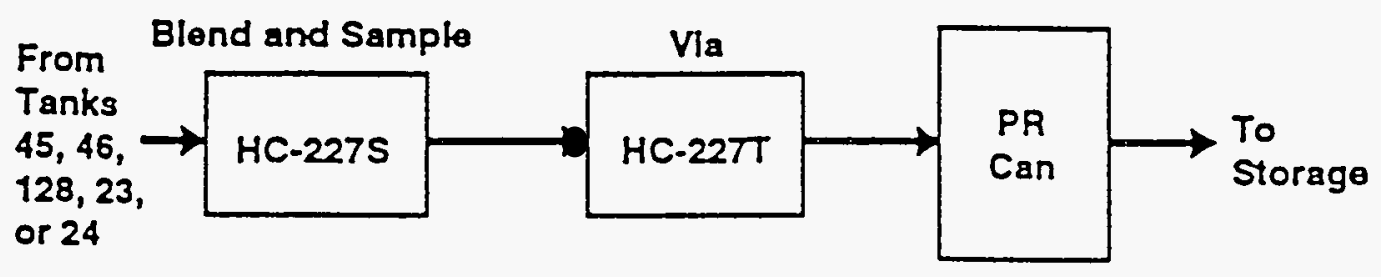


Figure 3-3. Remote Mechanical C Line Flow Diagram, Nitrate Solution Through Plutonium Fluoride Production.

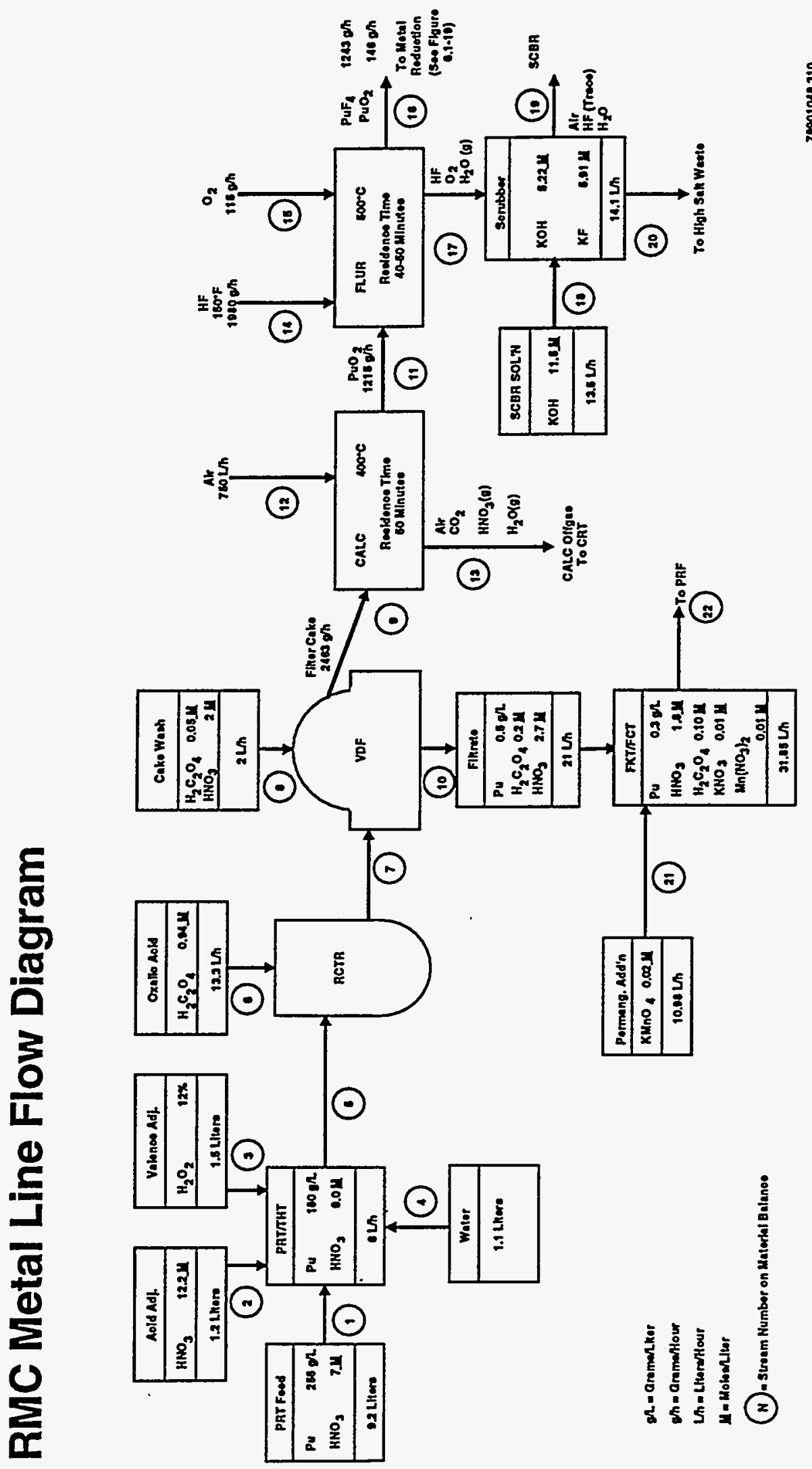


WHC-EP-0829

The RMC Line was semi-remotely operated from the RMC control room in room 229, adjacent to most of the RMC Line gloveboxes. Most of the operations in the gloveboxes starting from glovebox $\mathrm{HC}-7$ through $\mathrm{HC}-18 \mathrm{BS}$ (the button line) could be seen directly through the viewing windows of the control room.

\subsection{PLUTONIUH PROCESS SUPPORT LABORATORY}

The Plutonium Process Support Laboratory is located on the first floor of 234-5Z in the southwest corner of the building. Section 2.2.1 provides a detailed physical description of the 234-5Z Building.

The Plutonium Process Support Laboratory was chartered to operate nonradioactive and radioactive chemical-oriented demonstration laboratories and equipment to support numerous programs across the Hanford Site and, on occasion, other DOE Sites. as follows.

The specific laboratory rooms within the PFP and their capabilities are

- Room 179 contains a particle counter and a thermograviametric analysis instrument. The thermograviametric analys is instrument is located in a dedicated glovebox; three other gloveboxes and three hoods are available for general alpha-radioactive chemistry projects. One of the gloveboxes was occupied by and reserved for the TRU extraction process demonstration pilot plant.

- Room 180 contains the inductively coupled plasma spectrometer for rapid determinations of plutonium isotopes as well as other radioactive element determinations, including beta radioactive elements.

- Room 182 contains a scanning electron microscope for examining minute quantities of solids, sometimes alpha-radioactive.

- Room 183/185 was used for the scintillation counting equipment and waste drum storage.

- Room 187 contains one mobile ultraviolet/visible-light spectrometer used to identify unknown materials and five hoods for small, general, radioactive chemistry projects. Two of the hoods are connected to the radioactive D-4 drain 1 ine.

- Room 188 contains one glovebox for general alpha-radioactive chemistry projects.

- Room 189 contains a cold ion chromatograph, currently on a tabletop. Future plans include installing it in a glovebox for use with hot materials.

- Room 190 is a vault-type room used for storing stable SNM not currently needed for ongoing projects and demonstrations. 
- Room 191 is a nonradioactive chemistry Taboratory used for general chemistry projects and chemical preparation.

\subsection{ANALYTICAL LABORATORY}

The Analytical Laboratory, located on the first floor in the east end of the 234-5Z Building, was used to perform analytical and physical test analyses on plutonium samples. The samples came from the plant operations within the PFP, the research laboratory within PFP, and PUREX, as well as a few special test samples from other locations.

The Analytical Laboratory is located in rooms 131 through 157 of 234-5Z Building, with quality control standards being formulated and dispensed from rooms 221-C, 221-D, and 221-E. Section 2.2.1 provides a detailed physical description of the 234-5Z Building.

- Room 131 was used to analyze samples for plutonium concentration.

- Room 132 is the Mass Spectrometer Laboratory.

- Room 133 contains the grating and camera portions of a two-meter emission spectrograph and equipment for developing the film. No radioactive materials were allowed in this room.

- Room 134 was used to prepare samples for analysis on the mass spectrometer.

- Room 134-A was a weighing room used in support of the mass spectrometer laboratory.

- Room 135 was used to store and mount samples in support of the mass spectrometer laboratory.

- Room 136 contains a spectrophotometer.

- Room 137 contains the arc stand for the two-meter film spectrograph. These instruments were used in trace element analyses of plutonium and uranium product.

- Room 139 contains six interconnected gloveboxes. It was used to process and store all $\mathrm{PuO}_{2}$ and plutonium metal received by the laboratory for analysis.

- Room 141 was used to analyze cold samples that support the building process.

- Room 142 consists of four vented storage cabinets.

- Room 143 was used for decontamination of equipment for the Radiochemical Standards Laboratory, and the PFP Analytical Laboratory, handling of liquid waste not compatible with the waste recovery system, and disposal of LLW to the D-4 sump in 241-Z. 
- Work performed in room 144 provided the primary support for PRF and research and engineering plutonium samples. Among analyses performed were: plutonium concentration, visual percent solids, americium concentration, and acid/base determination.

- Room 145 contains a glovebox, which was used to analyze sinterability, bulk and tap density on $\mathrm{PuO}_{2}$, and for preparation of $\mathrm{PuO}_{2}$ standards for the emission spectrometer.

- Rooms 145-A, 147, 150, 151, and 148 are supply and office facilities.

- Room 149 contains an open-faced hood and a hood converted into a glovebox. Relatively large amounts of plutonium compounds were dissolved in the glovebox so the exhaust passes through a scrubber. The room was used for packing and unpacking shipping containers.

- Room 152 contains a load-in hood and a glovebox. It was used to reprocess 1 iquid 1 aboratory wastes.

- Room 153 consists of four open-faced hoods and one glovebox. The glovebox was used for surface area analys is of $\mathrm{PuO}_{2}$.

- Room 154 contains one glovebox and was used for support of $\mathrm{PuO}_{2}$. Carbon, sulfur, and nitrogen were analyzed in this glovebox.

- Room 155 contains four hoods and associated equipment that was used to support the PRF, RMA, and PUREX sample schedules.

- Room 156 was dedicated to uranium analyses and support of $\mathrm{UO}_{3}$ Facility operations.

- Room 157 consists of two gloveboxes with furnaces and a sample cell that were used to support $\mathrm{PuO}_{2}$ work.

- Room 146 was used for the analysis of plutonium solution and oxide material.

- Room 142 was a small room containing four vaults for radioactive standards storage.

- Room 143 has four open-faced hoods and one glovebox. The glovebox was used to precipitate low concentration plutonium nitrate and oxalate and filter.

Rooms 221-A through 221-G were used for the Recuplex process and were subjected to a criticality in 1962. The walls and floors were cleaned, but the crawl space between the ceiling and the duct level is still highly contaminated. No information is available regarding the contents of this crawl space. The pipeways in the tunnel level leading to the Recuplex have been sealed off and are also highty contaminated as a result of the same criticality. 
- Room 221-A (including 221-F and 221-G) is currently used for the Health Physics office.

- Room 221-B contains portable NDA equipment and containerized calibration/standard sources.

- Rooms 221-C, 221-D, and 221-E form the Radiochemical Standards Laboratory.

- Room 221-C contains a dry air glovebox used for the preparation of ultrapure $\mathrm{PuO}_{2}$ standards for the engineering laboritories.

- Room 221-D contains equipment used for makeup of plutonium sheet standards and soil standards and for doing plutonium nitrate characterization.

- Room 221-E contains equipment for the preparation of thorium and uranium standards. 
WHC-EP-0829

This page intentionally left blank.

$3-14$ 


\subsection{METHODOLOGY}

Estimates presented in this report of volumes and waste types that will result from the D\&D of the PFP are based on process and facility knowiedge, a detailed study of documentation (including $\mathrm{H}$ - series engineering drawings), and a walkdown. This section details the approach, methodology, uncertainties, and assumptions considered for this project. The results of this study are presented in Sections 5.0 and 6.0.

\subsection{UNCERTAINTIES}

The following uncertainties lead to the assumptions listed in Section 4.2 .

- In some cases engineering drawing documentation (H- series) was discontinued when processing was shutdown.

- Cleanout and changes subsequent to process shutdown have not been completely documented.

- Because of the historical nature of the processes at the PFP, some of the documentation was conflicting.

\subsection{ASSUMPTIONS}

It is essential that the reader understands the assumptions made during this project when interpreting or applying the results. The assumptions that apply globally are listed below. Specific assumptions are noted where they apply.

- Data gathered from documentation were assumed accurate when verification by walkdown was not possible.

- All uncontaminated solid waste will be removed prior to D\&D activities.

- Previously containerized radioactive and/or hazardous waste in temporary storage locations, such as hallways and vaults, will be removed prior to D\&D activities.

- Glovebox gloves will be removed and gloveports will be sealed prior to D\&D activities.

- All portable items within a glovebox that can be bagged out will be removed (e.g., hand tools, hot plates, HEPA filters, balances, pans). These items are not included in the volume estimates.

- GToveboxes shall remain intact. Glovebox volumes are based on the outside dimensions. 
- A7l nonportable items within a glovebox shall remain in the glovebox (e.g., tanks, process piping, manifolds). With the exception of process tanks, glovebox volumes shal1 include these items. Process tanks have been analyzed for hazardous chemicals and are treated separately to identify waste classification.

- GTovebox light fixtures will be removed prior to D\&D activities.

- Items that are either grouted or part of the fixed building structure will not be removed (e.g., nonload bearing walls, doors, equipment support columns, built-in filter racks).

- Alarm and safety support systems will remain in place (e.g., fire, industrial safety).

- Domestic systems will remain in place (e.g., sanitary systems, lighting).

- The most recent revision was given priority in cases of conflicting documentation.

- Areas without documentation regarding contamination were assumed to be uncontaminated.

- AT1 HEPA filters shall be removed from filterboxes and gloveboxes because the Waste Isolation Pilot Plant. will not accept them. The filterboxes will be included in the final D\&D solid waste volume. Permanent filter racks and filter rooms shall remain with the building structure.

\subsection{GENERAL APPROACH}

This study was designed to yield an estimate of the volumes and types of solid waste that will result from the D\&D of the PFP. A step-wise approach was used during this project. First, the facility was conservatively classified into three areas: "hot" process areas, "cold" process support areas, and uncontaminated areas. For the purposes of this study, "hot" process areas are defined as rooms in which any of the following conditions are met (most conservative for estimation).

- The room is in an area of potential airborne radioactivity according to Figure 8.3-8 in the Plutonium Finishing Plant Final Safety Analysis Report, HHC-SD-CP-SAR-021 (WHC 1991) (see Figure 4-1).

- The room has a normal dose level reading of greater than $2.0 \mathrm{mrem} / \mathrm{h}$ according to Table 8.4-1 and Figure 8.3-17 in WHC-SD-CP-SAR-021 (WHC 1991) (see Table 4-1 and Figure 4-2).

- The room has a radiation source within it according to figure 8.3-7 in WHC-SD-CP-SAR-021 (WHC 1991) (see Figure 4-3). 

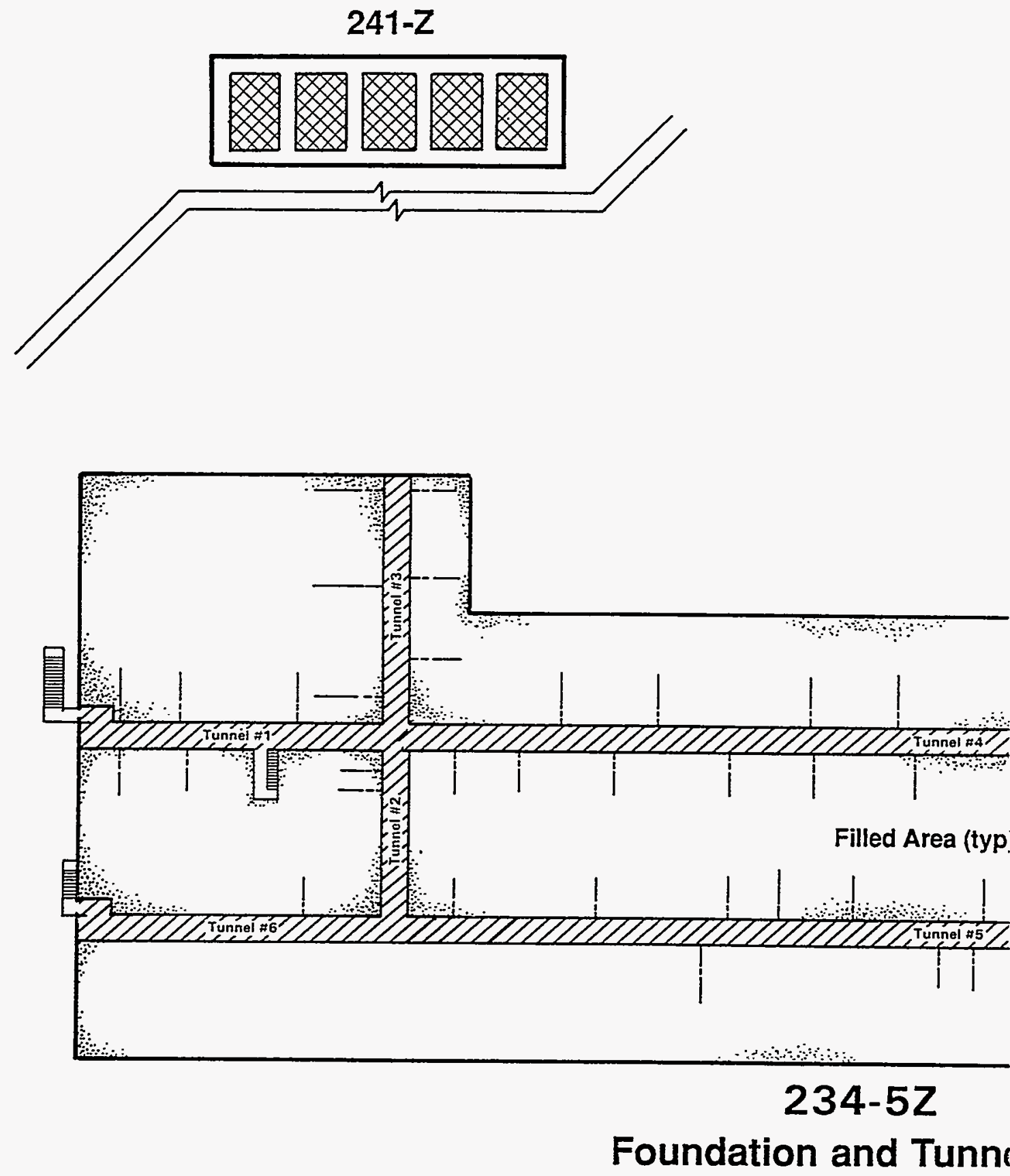
Figure 4-1. Plutonium Finishing $\mathrm{Pl}$ ant Ventilation Control and Radiation Control Areas. (sheet 1 of 5)

\section{Radiation Areas}

$\square$ Cold Clean Area

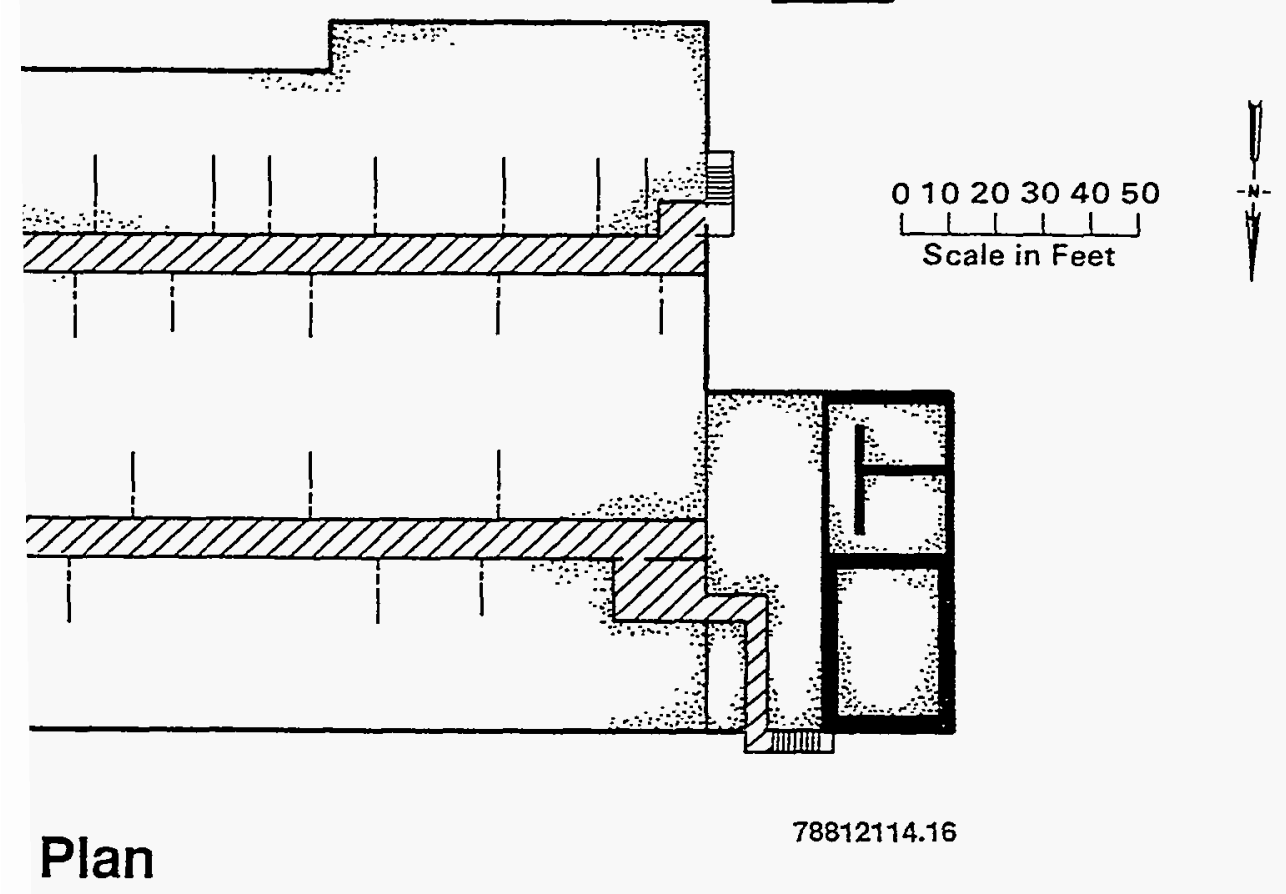




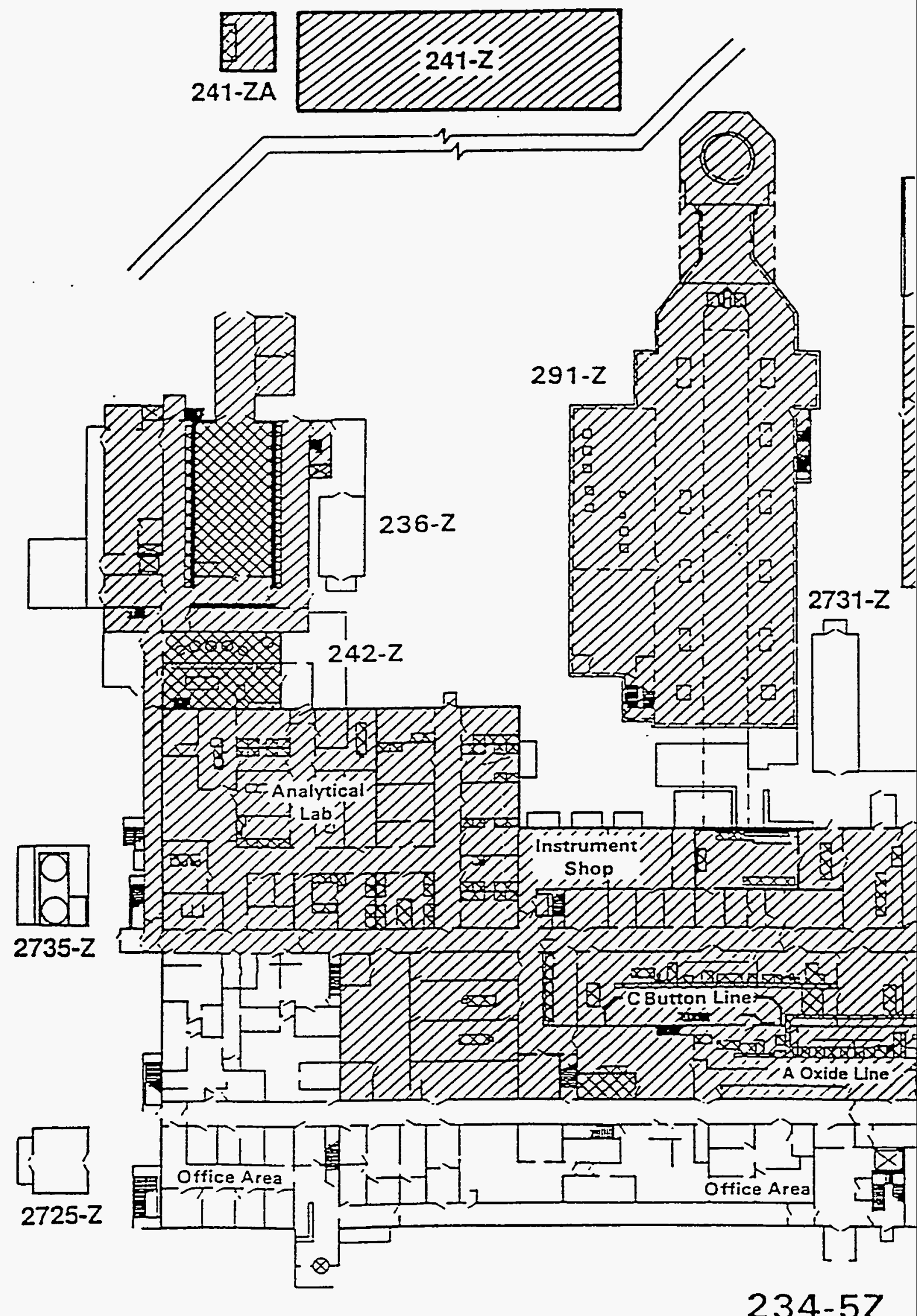

234-5Z

First Floor 
WHC-EP-0829

Figure 4-1. Plutonium Finishing Plant Ventilation Control and Radiation

Control Areas. (sheet 2 of 5)

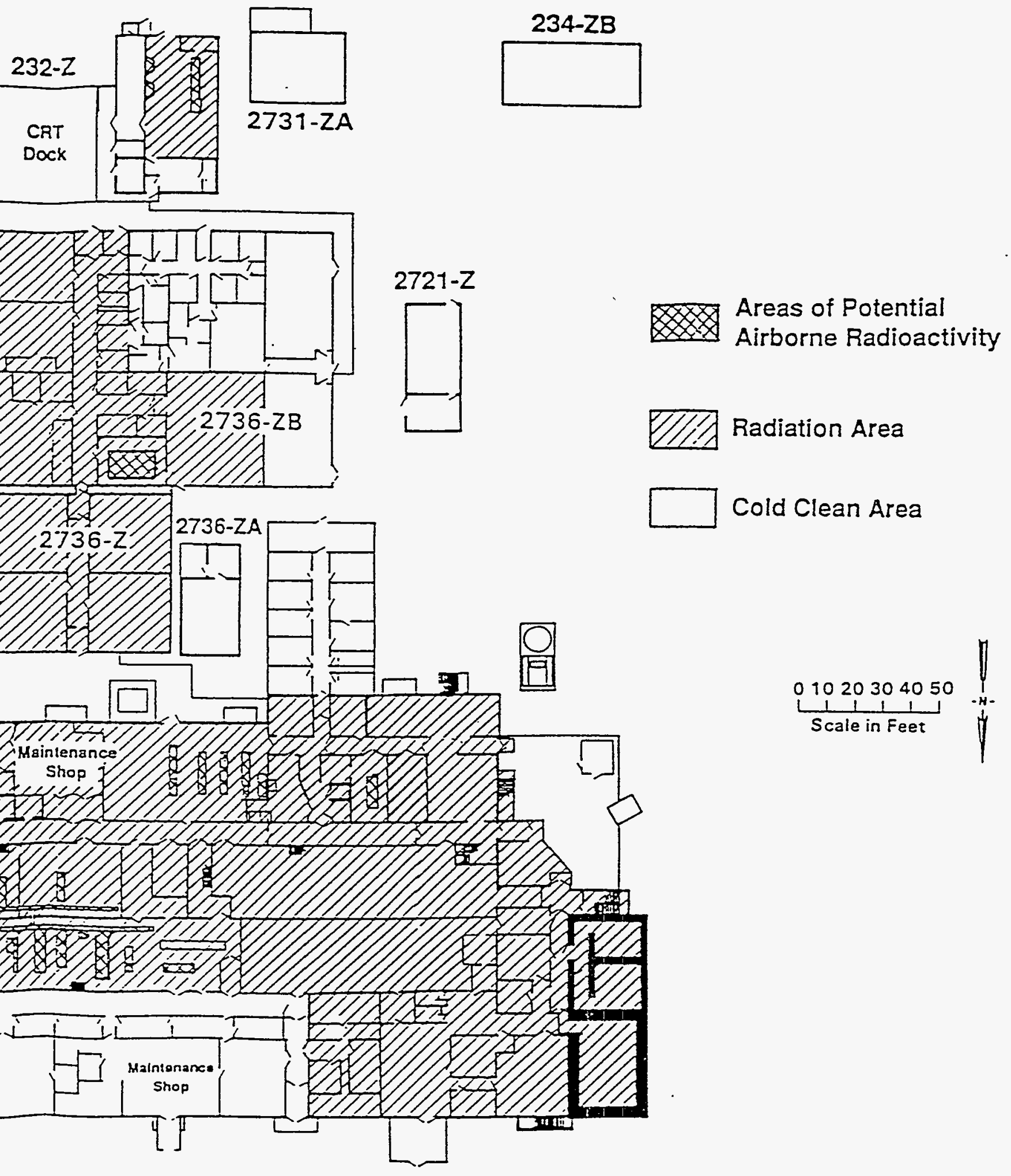

78812114.20 


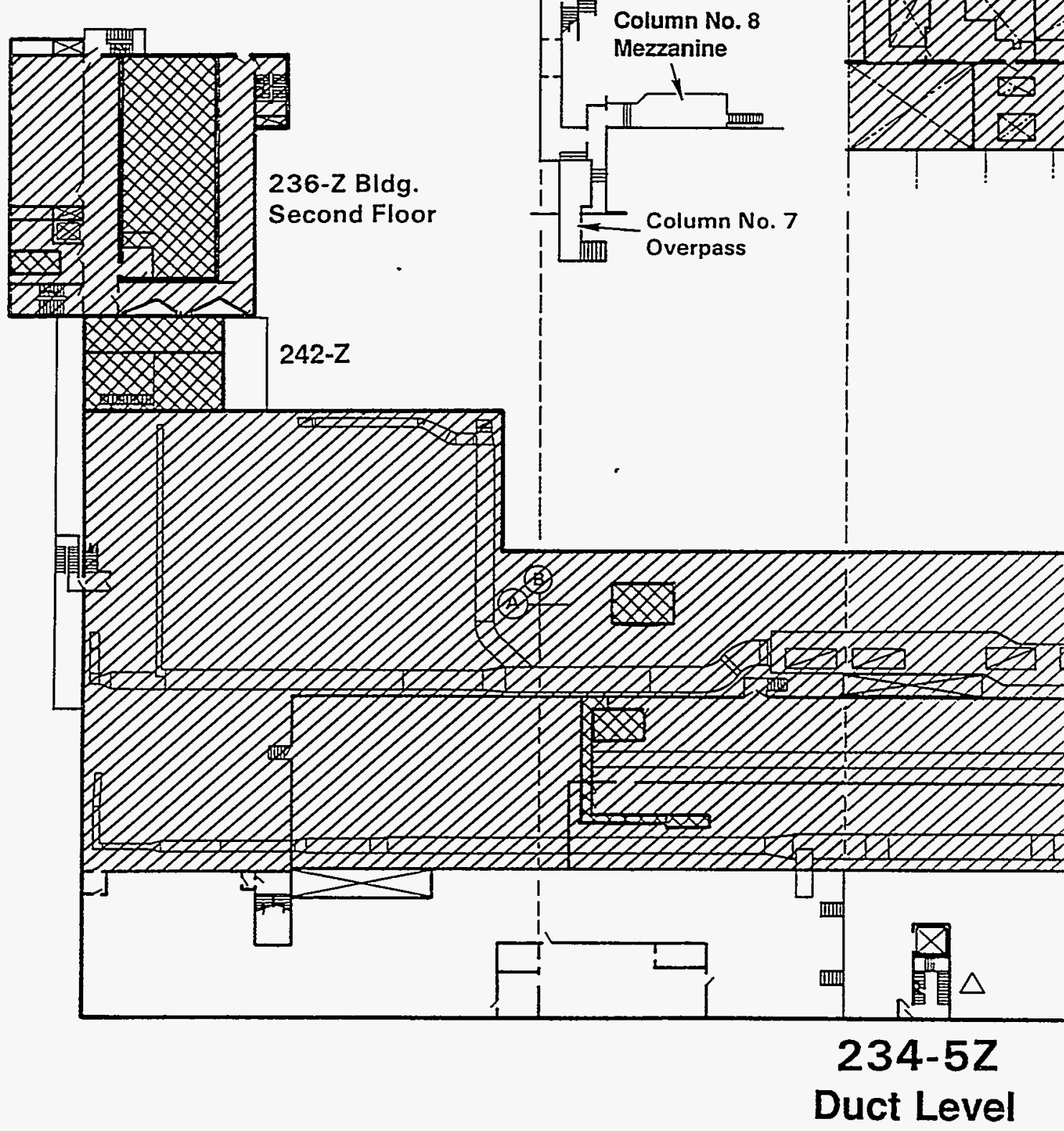




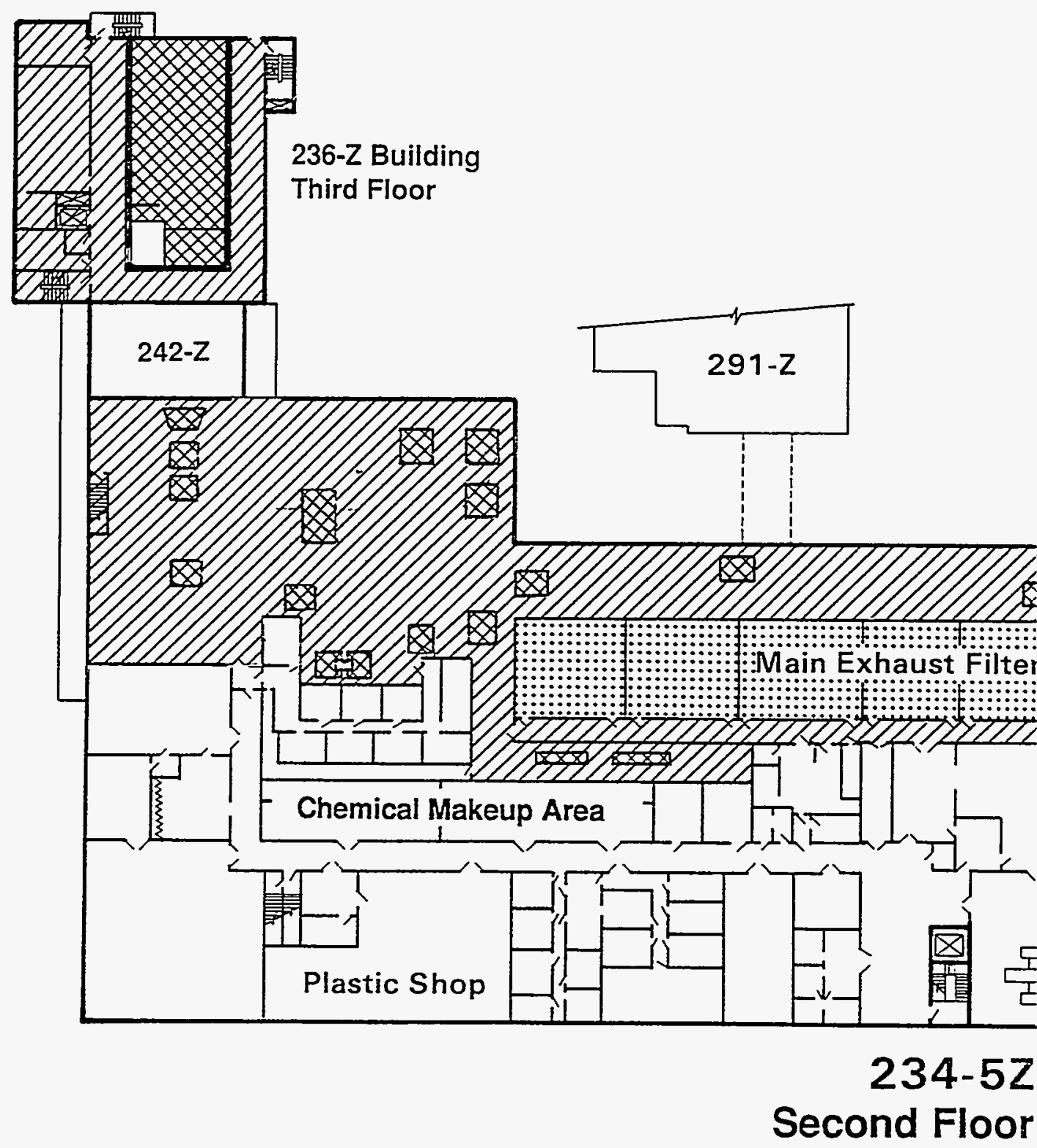


Figure 4-1. Plutonium Finishing Plant Ventilation Control and Radiation Control Areas. (sheet 4 of 5)

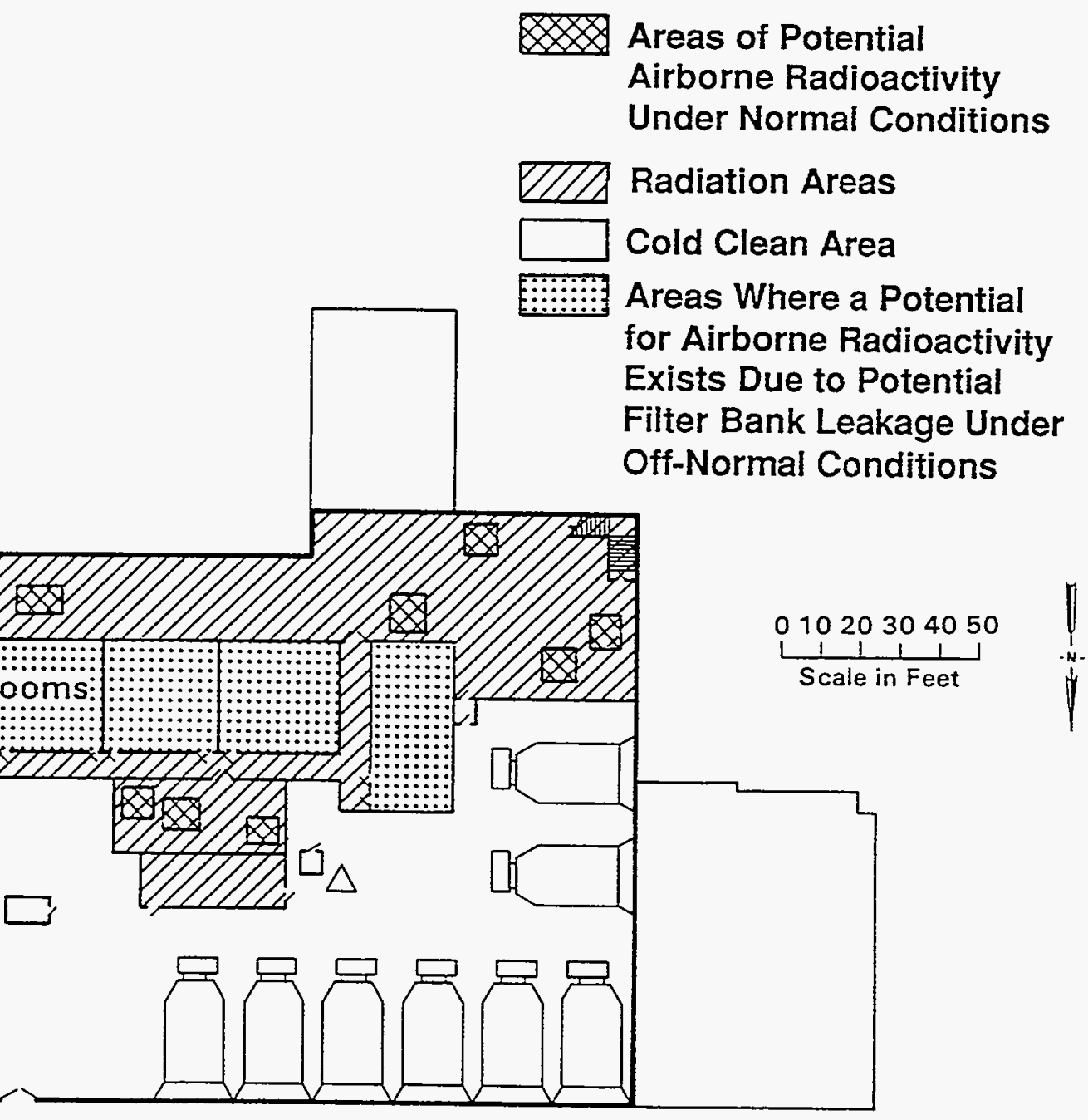

78812114.18

lan 


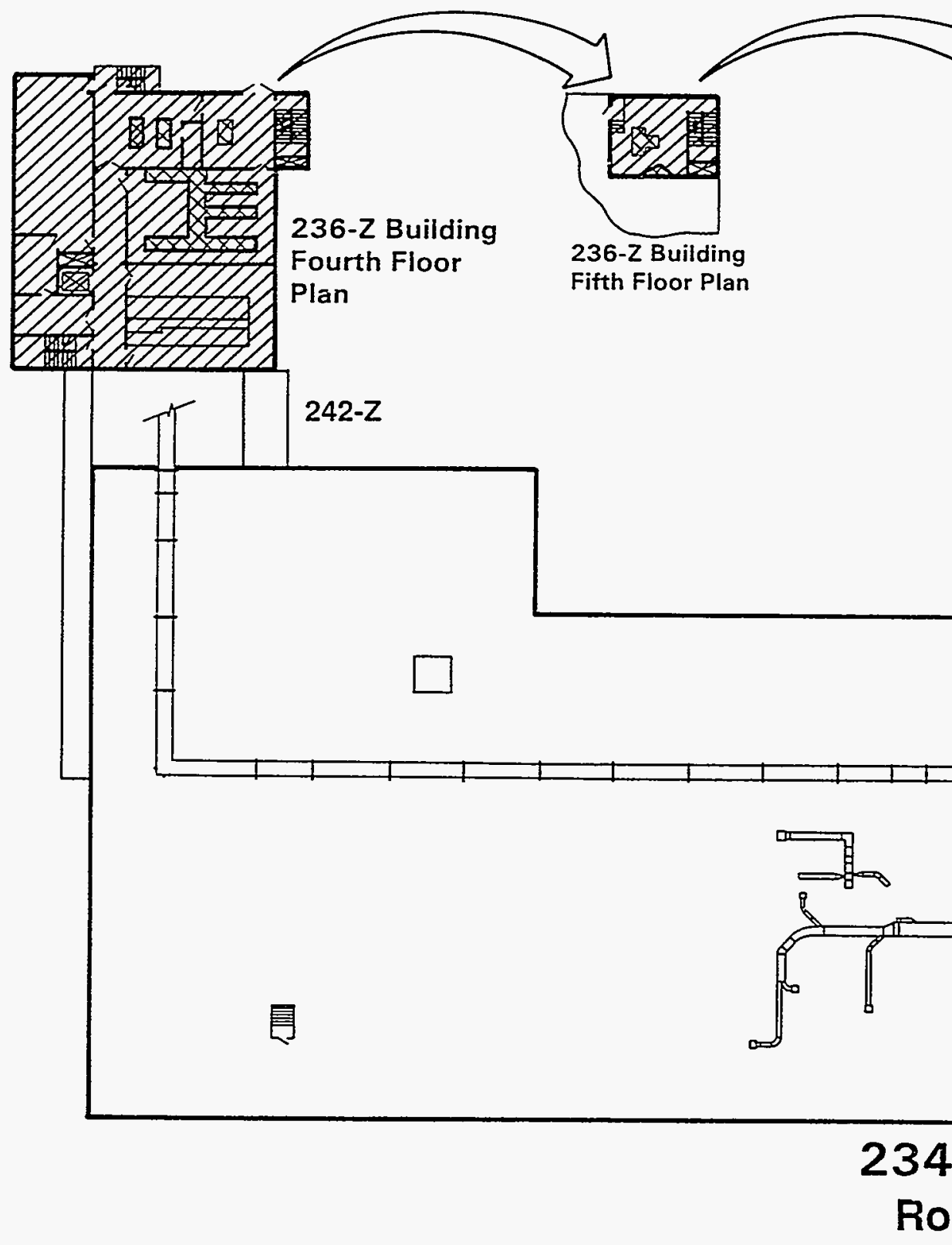


Figure 4-1. Plutonium Finishing Plant Ventilation Control and Radiation

Control Areas. (sheet 5 of 5)

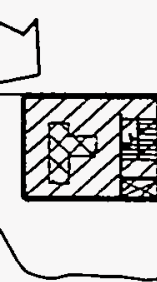

36-2 Building ixth Floor Plan

\section{Areas of Potential}

Airborne Radioactivity

Radiation Areas

Cold Clean Area

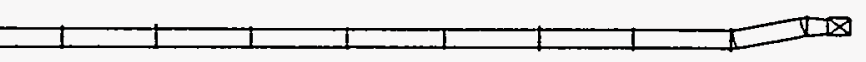


Table 4-1. Area Occupancy Factors of Plant Radiation or Potential Radiation Areas. (8 sheets)

\begin{tabular}{|c|c|c|c|}
\hline Room number & Operation/description & $\begin{array}{c}\text { Normal field } \\
\text { readings } \\
\text { (mrem } / \mathrm{h})\end{array}$ & $\begin{array}{c}\text { Area } \\
\text { occupancy }\end{array}$ \\
\hline \multicolumn{4}{|c|}{ 234-5Z Building } \\
\hline $100-A$ & RADTU filter room & $<2.0$ & $0.25 \mathrm{~h} / \mathrm{mo}$ \\
\hline $110-A$ & Homens' laundry hampers & $<0.5$ & $4 \mathrm{~h} / \mathrm{d}$ \\
\hline 112 & Men's rest room & $<0.5$ & Full time \\
\hline 116 & Men's changeroom & $<0.5$ & Full time \\
\hline 117 & Men's laundry hampers & $<0.5$ & $4 \mathrm{~h} / \mathrm{wk}$ \\
\hline 121 & Women's rest room & $<0.5$ & Full time \\
\hline 124 & Women's changeroom & $<0.5$ & Full time \\
\hline 126 & Personnel decontamination facility & $<0.5$ & $8 \mathrm{~h} / \mathrm{d}$ \\
\hline 131 & Plutonium assay & $<2.0$ & $16 \mathrm{~h} / \mathrm{d}$ \\
\hline 132 & Mass spectrometry & $<0.5$ & $16 \mathrm{~h} / \mathrm{d}$ \\
\hline 133 & Dark room & $<0.5$ & $1 \mathrm{~h} / \mathrm{wk}$ \\
\hline 134 & Mass spectrometry & $<0.5$ & $8 \mathrm{~h} / \mathrm{d}$ \\
\hline 135 & Mass spectrometry & $<0.5$ & $8 \mathrm{~h} / \mathrm{d}$ \\
\hline 136 & Emission spectrometry & $<0.5$ & $16 \mathrm{~h} / \mathrm{d}$ \\
\hline 137 & Emission spectrometry & $<0.5$ & $1 \mathrm{~h} / \mathrm{wk}$ \\
\hline 139 & $\mathrm{PuO}_{2}$ sample storage & $<30.0^{\mathrm{a}}$ & $16 \mathrm{~h} / \mathrm{d}$ \\
\hline $140-A$ & Analytical support services & $<0.5$ & $12 \mathrm{~h} / \mathrm{d}$ \\
\hline $140-B$ & Laboratory manager & $<0.5$ & $8 \mathrm{~h} / \mathrm{d}$ \\
\hline $140-C$ & Laboratory staff chemist & $<0.5$ & $8 \mathrm{~h} / \mathrm{d}$ \\
\hline 141 & Nonradioactive solution preparation & $<0.5$ & $2 \mathrm{~h} / \mathrm{d}$ \\
\hline 142 & Miscellaneous storage & $<2.0$ & $1 \mathrm{~h} / \mathrm{d}$ \\
\hline 143 & Waste process & $<3.0$ & $2 \mathrm{~h} / \mathrm{d}$ \\
\hline 144 & Process support & $<0.5$ & Ful1 time \\
\hline 145 & Physical test & $<2.0$ & Inactive \\
\hline $145-A$ & Stock room & $<0.5$ & $8 \mathrm{~h} / \mathrm{d}$ \\
\hline 146 & WIPP repackaging and sample analysis & $<0.5$ & $20 \mathrm{~h} / \mathrm{d}$ \\
\hline 147 & Waste packaging and analysis manager & $<0.5$ & $8 \mathrm{~h} / \mathrm{d}$ \\
\hline
\end{tabular}


Table 4-1. Area Occupancy Factors of Plant Radiation or Potential Radiation Areas. (8 sheets)

\begin{tabular}{|c|c|c|c|}
\hline Room number & Operation/description & $\begin{array}{l}\text { Normal field } \\
\text { readings } \\
\text { (mrem } / \mathrm{h})\end{array}$ & $\begin{array}{c}\text { Area } \\
\text { occupancy }\end{array}$ \\
\hline \multicolumn{4}{|c|}{ 234-5Z Building (cont.) } \\
\hline 148 & Sample analysis & $<0.5$ & $2 \mathrm{~h} / \mathrm{d}$ \\
\hline 149 & Sample dissolution & $<0.5$ & $<1 \mathrm{~h} / \mathrm{wk}$ \\
\hline 150 & Laboratory shift manager & $<0.5$ & $24 \mathrm{~h} / \mathrm{d}$ \\
\hline 151 & Miscellaneous counting room & $<0.5$ & $24 \mathrm{~h} / \mathrm{d}$ \\
\hline 152 & Waste concentrator & $<2.0$ & $4 \mathrm{~h} / \mathrm{d}$ \\
\hline 153 & Product specification analysis & $<1.5$ & $4 \mathrm{~h} / \mathrm{d}$ \\
\hline 154 & Product specification analysis & $<0.5$ & $8 \mathrm{~h} / \mathrm{d}$ \\
\hline 155 & Infrared spectrophotometer & $<0.5$ & $2 \mathrm{~h} / \mathrm{d}$ \\
\hline 156 & S\&C analysis & $<0.5$ & $4 \mathrm{~h} / \mathrm{d}$ \\
\hline 157 & Product specification analysis & $<0.5$ & $<1 \mathrm{~h} / \mathrm{wk}$ \\
\hline 158 & Instrument shop & $<0.5$ & Full time \\
\hline 159 & Instrument shop & $<0.5$ & Full time \\
\hline $159-A$ & Instrument shop & $<0.5$ & $4 \mathrm{~h} / \mathrm{d}$ \\
\hline 161 & RMC shift manager & $<0.5$ & Furl time \\
\hline 162 & RMC shift manager & $<0.5$ & $20 \mathrm{~h} / \mathrm{d}$ \\
\hline 163 & Instruments manager & $<0.5$ & $8 \mathrm{~h} / \mathrm{d}$ \\
\hline 164 & RMC shift office & $<0.5$ & Full time \\
\hline 165 & Calcium storage & $<0.5$ & Full time \\
\hline 166 & RMC batch tanks & $<30.0^{a}$ & $4 \mathrm{~h} / \mathrm{d}$ \\
\hline 167 & RMC operations storage & $<0.5$ & Full time \\
\hline 168 & RMC ready room/storage & $<0.5$ & Full time \\
\hline 169 & Ash stabilization & $<2.0$ & $16 \mathrm{~h} / \mathrm{d}$ \\
\hline 170 & Ash dissolution & $<0.5$ & $<1 \mathrm{~h} / \mathrm{wk}$ \\
\hline 171 & Corridor & $<0.5$ & Full time \\
\hline 172 & Maintenance shop (RMC) & $<2.0$ & Full time \\
\hline 173 & Maintenance shop & $<2.0$ & Full time \\
\hline 174 & SNM storage vault & $<30.0$ & $6 \mathrm{~h} / \mathrm{wk}$ \\
\hline
\end{tabular}


Table 4-1. Area Occupancy Factors of P1ant Radiation or Potential Radiation Areas. (8 sheets)

\begin{tabular}{|c|c|c|c|}
\hline Room number & Operation/description & $\begin{array}{c}\text { Normal field } \\
\text { readings } \\
\text { (mrem } / h \text { ) }\end{array}$ & $\begin{array}{c}\text { Area } \\
\text { occupancy }\end{array}$ \\
\hline \multicolumn{4}{|c|}{ 234-5Z Building (cont.) } \\
\hline 175 & SNM storage vault & $<2.0$ & $6 \mathrm{~h} / \mathrm{wk}$ \\
\hline 179 & Process support laboratory & $<0.5$ & $8 \mathrm{~h} / \mathrm{d}$ \\
\hline 180 & Isotopic research & $<0.5$ & $8 \mathrm{~h} / \mathrm{d}$ \\
\hline 182 & Process support (microscope) & $<0.5$ & $8 \mathrm{~h} / \mathrm{d}$ \\
\hline 183 & Specialized processing & $<0.5$ & $8 \mathrm{~h} / \mathrm{d}$ \\
\hline 185 & Miscellaneous storage & $<0.5$ & $8 \mathrm{~h} / \mathrm{d}$ \\
\hline 187 & Open-faced hoods & $<0.5$ & $8 \mathrm{~h} / \mathrm{d}$ \\
\hline 188 & Glovebox/chemical storage & $<0.5$ & $8 \mathrm{~h} / \mathrm{d}$ \\
\hline 189 & Equipment storage & $<0.5$ & $8 \mathrm{~h} / \mathrm{d}$ \\
\hline 190 & Radioactive material storage & $<30.0$ & $1 \mathrm{~h} / \mathrm{d}$ \\
\hline 191 & Product support cold laboratory & $<0.5$ & $3 \mathrm{~h} / \mathrm{wk}$ \\
\hline 192 & Drum staging area & $<0.5$ & $4 \mathrm{~h} / \mathrm{d}$ \\
\hline $192-A$ & SNM storage vault & $<30.0^{\mathrm{a}}$ & $1 \mathrm{~h} / \mathrm{wk}$ \\
\hline 192-B & SNM storage vault & $>30.0^{\mathrm{a}}$ & $1 \mathrm{~h} / \mathrm{wk}$ \\
\hline $192-C$ & SNM storage vault & $>30.0^{\mathrm{a}}$ & $1 \mathrm{~h} / \mathrm{wk}$ \\
\hline 192-D & PR can storage & $<0.5$ & $1 \mathrm{~h} / \mathrm{wk}$ \\
\hline $192-S$ & Storage & $<0.5$ & $1 \mathrm{~h} / \mathrm{wk}$ \\
\hline $192-W$ & NDA storage & $<0.5$ & $0.25 \mathrm{~h} / \mathrm{wk}$ \\
\hline 193 & Airlock & $<0.5$ & $0.25 \mathrm{~h} / \mathrm{wk}$ \\
\hline 196 & Waste drum storage & $<0.5$ & $0.25 \mathrm{~h} / \mathrm{wk}$ \\
\hline 197 & Waste drum storage & $<0$ & $0.25 \mathrm{~h} / \mathrm{wk}$ \\
\hline $197-A$ & Mixed waste drum storage & $<0.5$ & $0.25 \mathrm{~h} / \mathrm{wk}$ \\
\hline $221-A$ & HP office & $<0.5$ & $24 \mathrm{~h} / \mathrm{d}$ \\
\hline 221-B & Sealer repair shop & $<0.5$ & $4 \mathrm{~h} / \mathrm{wk}$ \\
\hline $221-C$ & Radiochemical standards laboratory & $<2.0$ & $8 \mathrm{~h} / \mathrm{d}$ \\
\hline $221-D$ & Radiochemical standards laboratory & $<2.0$ & $8 \mathrm{~h} / \mathrm{d}$ \\
\hline $221-E$ & Radiochemical standards laboratory & $<2.0$ & $8 \mathrm{~h} / \mathrm{d}$ \\
\hline
\end{tabular}


Table 4-1. Area Occupancy Factors of Plant Radiation or Potential Radiation Areas. (8 sheets)

\begin{tabular}{|c|c|c|c|}
\hline Room number & Operation/description & $\begin{array}{l}\text { Normal field } \\
\text { readings } \\
(\mathrm{mrem} / \mathrm{h})\end{array}$ & $\begin{array}{c}\text { Area } \\
\text { occupancy }\end{array}$ \\
\hline \multicolumn{4}{|c|}{ 234-5Z Building (cont.) } \\
\hline 225 & Product handling vault & $>30.0^{\mathrm{a}}$ & $1 \mathrm{~h} / \mathrm{wk}$ \\
\hline 227 & Product LI/LO & $<2.0$ & $8 \mathrm{~h} / \mathrm{wk}$ \\
\hline $228-A$ & RMC production line & $<30.0^{\mathrm{a}}$ & Full time \\
\hline $228-B$ & RMC production line & $<30.0^{\mathrm{a}}$ & Full time \\
\hline $228-C$ & RMC production line & $<30.0^{\mathrm{a}}$ & Full time \\
\hline $230-A$ & RMC seal-out glovebox & $<30.0^{\mathrm{a}}$ & $4 \mathrm{~h} / \mathrm{wk}$ \\
\hline $230-B$ & RMC recoverable powder glovebox & $<30.0^{a}$ & $4 \mathrm{~h} / \mathrm{wk}$ \\
\hline $230-C$ & GTovebox HC-60 hydrolysis & $<2.0$ & $4 \mathrm{~h} / \mathrm{d}$ \\
\hline 232 & HF scrubber cell & $<2.0$ & Full time \\
\hline 233 & RMA control room & $<2.0$ & $8 \mathrm{~h} / \mathrm{d}$ \\
\hline 234 & Trash compactor & $<2.0$ & $1 \mathrm{~h} / \mathrm{wk}$ \\
\hline $234-A$ & NDA measurement of drums & $<2.0$ & $4 \mathrm{~h} / \mathrm{wk}$ \\
\hline $235-A 1$ & RMA oxide line (inactive) & $>30.0^{\mathrm{a}}$ & $1 \mathrm{~h} / \mathrm{wk}$ \\
\hline $235-A 2$ & RMA oxide line (inactive) & $<30.0^{\mathrm{a}}$ & $1 \mathrm{~h} / \mathrm{wk}$ \\
\hline $235-A 3$ & RMA oxide line (inactive) & $<30.0^{\mathrm{a}}$ & $1 \mathrm{~h} / \mathrm{wk}$ \\
\hline $235-B$ & Recoverable material stabilization & $<30.0^{\mathrm{a}}$ & $8 \mathrm{~h} / \mathrm{d}$ \\
\hline $235-C$ & Waste repackaging & $<1.0$ & $8 \mathrm{~h} / \mathrm{d}$ \\
\hline $235-D$ & Waste drum storage & $<2.0$ & $4 \mathrm{~h} / \mathrm{wk}$ \\
\hline 236 & PR can storage vault & $<30.0^{\mathrm{a}}$ & $2 \mathrm{~h} / \mathrm{wk}$ \\
\hline 245 & Corridor & $<0.5$ & $0.5 \mathrm{~h} / \mathrm{wk}$ \\
\hline 262 & Duct leve1 & $<2.0$ & $4 \mathrm{~h} / \mathrm{d}$ \\
\hline 263 & Duct level & $<2.0$ & $4 \mathrm{~h} / \mathrm{d}$ \\
\hline 264 & Duct level & $<2.0$ & $4 \mathrm{~h} / \mathrm{d}$ \\
\hline 308 & Duct level & $<0.5$ & $4 \mathrm{~h} / \mathrm{d}$ \\
\hline 309 & E-4 filter room & $<0.5$ & $1 \mathrm{~d} / \mathrm{yr}$ \\
\hline 310 & E-4 filter room & $<0.5$ & $1 \mathrm{~d} / \mathrm{yr}$ \\
\hline 311 & E-3 filter room & $<0.5$ & $1 d / y r$ \\
\hline
\end{tabular}


Table 4-1. Area Occupancy Factors of Plant Radiation or Potential Radiation Areas. ( 8 sheets)

\begin{tabular}{|c|c|c|c|}
\hline Room number & Operation/description & \begin{tabular}{|c|} 
Normal field \\
readings \\
(mrem/h)
\end{tabular} & $\begin{array}{c}\text { Area } \\
\text { occupancy }\end{array}$ \\
\hline \multicolumn{4}{|c|}{ 234-5Z Building (cont.) } \\
\hline 312 & E-3 filter room & $<0.5$ & $1 \mathrm{~d} / \mathrm{yr}$ \\
\hline 313 & E-3 filter room & $<0.5$ & $1 \mathrm{~d} / \mathrm{yr}$ \\
\hline 314 & E-3 filter room & $<0.5$ & $1 \mathrm{~d} / \mathrm{yr}$ \\
\hline 315 & E-3 filter room & $<0.5$ & $<1 d / y r$ \\
\hline 316 & E-3 filter room & $<0.5$ & $<1 d / y r$ \\
\hline 318 & E-3 filter room & $<0.5$ & $<1 \mathrm{~d} / \mathrm{yr}$ \\
\hline 320 & Filterboxes & $<2.0$ & $<1 d / y r$ \\
\hline \multicolumn{4}{|c|}{ 2736-ZB Building } \\
\hline 616 & Homen's changeroom & $<0.5$ & $4 \mathrm{~h} / \mathrm{wk}$ \\
\hline 622 & Men's changeroom & $<0.5$ & $4 \mathrm{~h} / \mathrm{wk}$ \\
\hline 623 & UPS room & $<0.5$ & $0.25 \mathrm{~h} / \mathrm{wk}$ \\
\hline 624 & Corridor & $<0.5$ & $0.5 \mathrm{~h} / \mathrm{wk}$ \\
\hline 625 & Corridor & $<0.5$ & $0.5 \mathrm{~h} / \mathrm{wk}$ \\
\hline 626 & Janitor closet & $<0.5$ & $0.25 \mathrm{~h} / \mathrm{wk}$ \\
\hline 627 & Homen's rest room & $<0.5$ & $1 \mathrm{~h} / \mathrm{wk}$ \\
\hline 628 & Men's rest room & $<0.5$ & $1 \mathrm{~h} / \mathrm{wk}$ \\
\hline 629 & Mardix & $<0.5$ & $9 \mathrm{~h} / \mathrm{d}$ \\
\hline 630 & Decontamination room & $<0.5$ & $0.25 \mathrm{~h} / \mathrm{wk}$ \\
\hline 631 & HP office & $<0.5$ & $4 \mathrm{~h} / \mathrm{d}$ \\
\hline 632 & Applied technology chemist's office & $<0.5$ & $6 \mathrm{~h} / \mathrm{d}$ \\
\hline 633 & Corridor & $<0.5$ & $0.5 \mathrm{~h} / \mathrm{wk}$ \\
\hline 634 & Shift manager & $<0.5$ & $2 \mathrm{~h} / \mathrm{d}$ \\
\hline 635 & Operator ready room & $<0.5$ & $2 \mathrm{~h} / \mathrm{d}$ \\
\hline 636 & Repackaging glovebox & $<30.0^{\mathrm{a}}$ & $0.5 \mathrm{~h} / \mathrm{wk}$ \\
\hline 637 & NDA laboratory & $<30.0^{\mathrm{a}}$ & $8 \mathrm{~h} / \mathrm{d}$ \\
\hline 638 & Packaging glovebox & $<30.0^{8}$ & $6 \mathrm{~h} / \mathrm{wk}$ \\
\hline 639 & Safety shower room & $<30.0^{\mathrm{a}}$ & $0.25 \mathrm{~h} / \mathrm{wk}$ \\
\hline
\end{tabular}


Table 4-1. Area Occupancy Factors of Plant Radiation or Potential Radiation Areas. (8 sheets)

\begin{tabular}{|c|c|c|c|}
\hline Room number & Operation/description & $\begin{array}{c}\text { Normal field } \\
\text { readings } \\
(\text { mrem } / \mathrm{h})\end{array}$ & $\begin{array}{c}\text { Area } \\
\text { occupancy }\end{array}$ \\
\hline \multicolumn{4}{|c|}{ 2736-ZB Building (cont.) } \\
\hline 640 & Supply storage & $<30.0^{a}$ & $0.25 \mathrm{~h} / \mathrm{wk}$ \\
\hline 641 & Receiving room & $<30.0^{\circ}$ & $2 \mathrm{~h} / \mathrm{wk}$ \\
\hline 642 & Shipping room & $<30.0^{8}$ & $2 \mathrm{~h} / \mathrm{wk}$ \\
\hline 643 & Personnel entry & $<0.5$ & $0.25 \mathrm{~h} / \mathrm{wk}$ \\
\hline 644 & Material passageway & $<0.5$ & $0.25 \mathrm{~h} / \mathrm{wk}$ \\
\hline \multicolumn{4}{|c|}{ 236-Z Building } \\
\hline 10 & Corridor/filterboxes & $<10.0$ & $6 \mathrm{~h} / \mathrm{wk}$ \\
\hline 11 & Corridor & $<30.0^{\mathrm{a}}$ & $3 \mathrm{~h} / \mathrm{d}$ \\
\hline 12 & Process cell & $>30.0^{\mathrm{B}}$ & $1 \mathrm{~d} / \mathrm{yr}$ \\
\hline 13 & Corridor & $<30.0^{\mathrm{a}}$ & $4 \mathrm{~h} / \mathrm{d}$ \\
\hline 14 & Corridor & $<30.0^{\circ}$ & $4 \mathrm{~h} / \mathrm{wk}$ \\
\hline 15 & Welding shop & $<0.5$ & $1 \mathrm{~h} / \mathrm{wk}$ \\
\hline 16 & Maintenance shop (PRF) & $<0.5$ & $8 \mathrm{~h} / \mathrm{d}$ \\
\hline 17 & Maintenance manager's office & $<0.5$ & $8 \mathrm{~h} / \mathrm{d}$ \\
\hline 18 & Elevator access/storage & $<0.5$ & $2 \mathrm{~h} / \mathrm{wk}$ \\
\hline 19 & NDA counting room & $<0.5$ & $1 \mathrm{~h} / \mathrm{d}$ \\
\hline 20 & Corridor/filterboxes & $<2.0$ & $4 \mathrm{~h} / \mathrm{wk}$ \\
\hline 21 & Hest corridor & $<2.0$ & $4 \mathrm{~h} / \mathrm{wk}$ \\
\hline 25 & East corridor & $<2.0$ & $4 \mathrm{~h} / \mathrm{wk}$ \\
\hline 26 & Filter room & $<0.5$ & $1 \mathrm{~h} / \mathrm{wk}$ \\
\hline 27 & Maintenance glovebox & $<30.0^{a}$ & $2 \mathrm{~h} / \mathrm{d}$ \\
\hline 30 & Corridor & $<2.0$ & $1 \mathrm{~h} / \mathrm{d}$ \\
\hline 31 & West corridor & $<2.0$ & $\mathrm{I} \mathrm{h} / \mathrm{d}$ \\
\hline 33 & East corridor & $<0.5$ & $1 \mathrm{~h} / \mathrm{d}$ \\
\hline 34 & Instrument shop & $<0.5$ & $2 \mathrm{~h} / \mathrm{d}$ \\
\hline 35 & Electrical room & $<0.5$ & $0.5 \mathrm{~h} / \mathrm{wk}$ \\
\hline 36 & Compressor room & $<0.5$ & $1 \mathrm{~h} / \mathrm{wk}$ \\
\hline
\end{tabular}


Table 4-1. Area 0ccupancy Factors of Plant Radiation or Potential Radiation Areas. (8 sheets)

\begin{tabular}{|c|c|c|c|}
\hline Room number & Operation/description & $\begin{array}{l}\text { Normal field } \\
\text { readings } \\
(\mathrm{mrem} / \mathrm{h})\end{array}$ & $\begin{array}{c}\text { Area } \\
\text { occupancy }\end{array}$ \\
\hline \multicolumn{4}{|c|}{ 236-Z Building (cont.) } \\
\hline 37 & Storage room & $<0.5$ & $1 \mathrm{~h} / \mathrm{wk}$ \\
\hline 38 & Rest room & $<0.5$ & $4 \mathrm{~h} / \mathrm{wk}$ \\
\hline 40 & Chemical preparation & $<0.5$ & $3 \mathrm{~h} / \mathrm{d}$ \\
\hline 41 & MT & $<30.0^{\mathrm{a}}$ & $24 \mathrm{~h} / \mathrm{d}$ \\
\hline 42 & Column room & $<30.0^{\mathrm{a}}$ & $0.25 \mathrm{~h} / \mathrm{d}$ \\
\hline 43 & S\&C gloveboxes & $<2.0$ & $0.25 \mathrm{~h} / \mathrm{d}$ \\
\hline 44 & Control room & $<0.5$ & $24 \mathrm{~h} / \mathrm{d}$ \\
\hline 45 & Shift manager's office & $<0.5$ & $24 \mathrm{~h} / \mathrm{d}$ \\
\hline 46 & Shift engineer's office & $<0.5$ & $24 \mathrm{~h} / \mathrm{d}$ \\
\hline 47 & Corridor & $<0.5$ & $0.25 \mathrm{~h} / \mathrm{d}$ \\
\hline 50 & Column glovebox & $<2.0$ & $0.5 \mathrm{~h} / \mathrm{d}$ \\
\hline 60 & Column glovebox & $<30.0$ & $0.5 \mathrm{~h} / \mathrm{d}$ \\
\hline \multicolumn{4}{|c|}{ 291-Z Building } \\
\hline 500 & Electrical room & $<0.5$ & $4 \mathrm{~h} / \mathrm{wk}$ \\
\hline 501 & Compressor room & $<0.5$ & $4 \mathrm{~h} / \mathrm{wk}$ \\
\hline 502 & Exhaust plenum & $<0.5$ & 0 \\
\hline 509 & Fan room & $<0.5$ & $2 \mathrm{~h} / \mathrm{wk}$ \\
\hline \multicolumn{4}{|c|}{ 232-Z Building } \\
\hline Operating area & Storage & $<2.0$ & $0.5 \mathrm{~h} / \mathrm{wk}$ \\
\hline Changeroom & Laundry (SWPs) & $<0.5$ & $1 \mathrm{~h} / \mathrm{wk}$ \\
\hline Storage area & Used filters & $<0.5$ & $0.25 \mathrm{~h} / \mathrm{wk}$ \\
\hline \multicolumn{4}{|c|}{ 2736-Z Building } \\
\hline 1 & Storage of product/recoverable SNM & $<30.0$ & $1.5 \mathrm{~h} / \mathrm{wk}^{\mathrm{b}}$ \\
\hline 2 & Lard can/cubicle SNM storage & $>30.0$ & $1.5 \mathrm{~h} / \mathrm{wk}^{\mathrm{b}}$ \\
\hline 3 & Storage of product/recoverable SNM & $>30.0$ & $1.5 \mathrm{~h} / \mathrm{wk}^{\mathrm{b}}$ \\
\hline 4 & Storage of product/recoverable SNM & $>30.0$ & $1.5 \mathrm{~h} / \mathrm{wk}^{\mathrm{b}}$ \\
\hline
\end{tabular}


Table 4-1. Area Occupancy Factors of Plant Radiation or Potential Radiation Areas. ( 8 sheets)

\begin{tabular}{|c|c|c|c|}
\hline Room number & Operation/description & $\begin{array}{c}\text { Normal field } \\
\text { readings } \\
(\text { mrem } / \mathrm{h})\end{array}$ & $\begin{array}{c}\text { Area } \\
\text { occupancy }\end{array}$ \\
\hline \multicolumn{4}{|c|}{ 241-Z/ZA Building } \\
\hline Sumps & Sump tank waste storage & $<30.0$ & $1 \mathrm{~d} / 2 \mathrm{yr}$ \\
\hline 241-Z Building & Ventilation/operating area & $<0.5$ & $0.25 \mathrm{~h} / \mathrm{d}$ \\
\hline 241-Z Building & Sampling glovebox & $<2.0$ & $1 \mathrm{~h} / \mathrm{wk}$ \\
\hline
\end{tabular}

NOTE: The $\mathrm{mrem} / \mathrm{h}$ values are gamma $(Q F=1)$ plus neutron exposure rates.

a Dose rate is associated with the principal work station in the area indicated. General area readings are usually 10 percent or less of this value. The area occupancy is as indicated, but work station occupancy is much lower.

On an as-needed basis. 

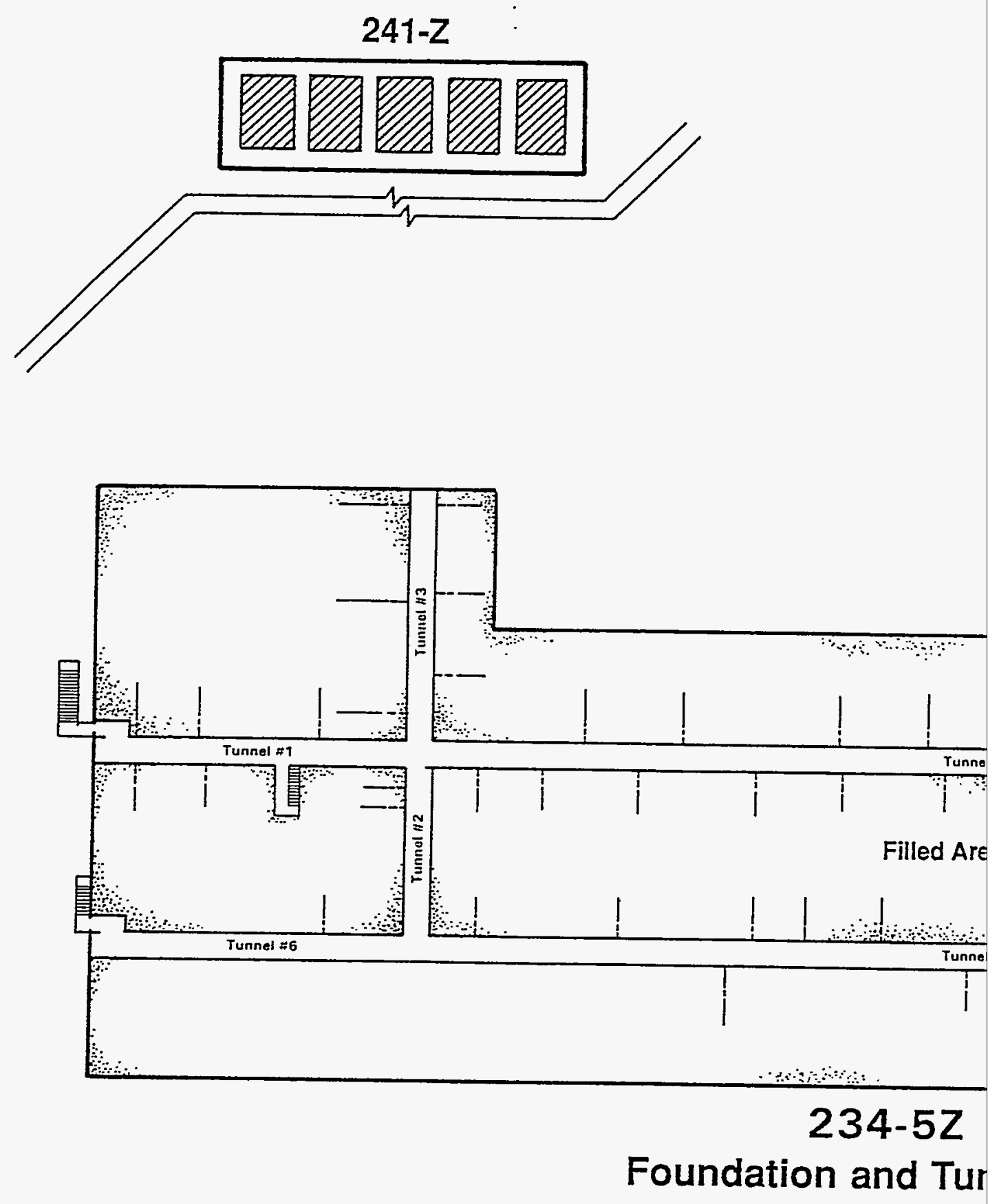
Figure 4-2. Plutonium Finishing Plant Radiation Dose Levels. (sheet 1 of 5 )

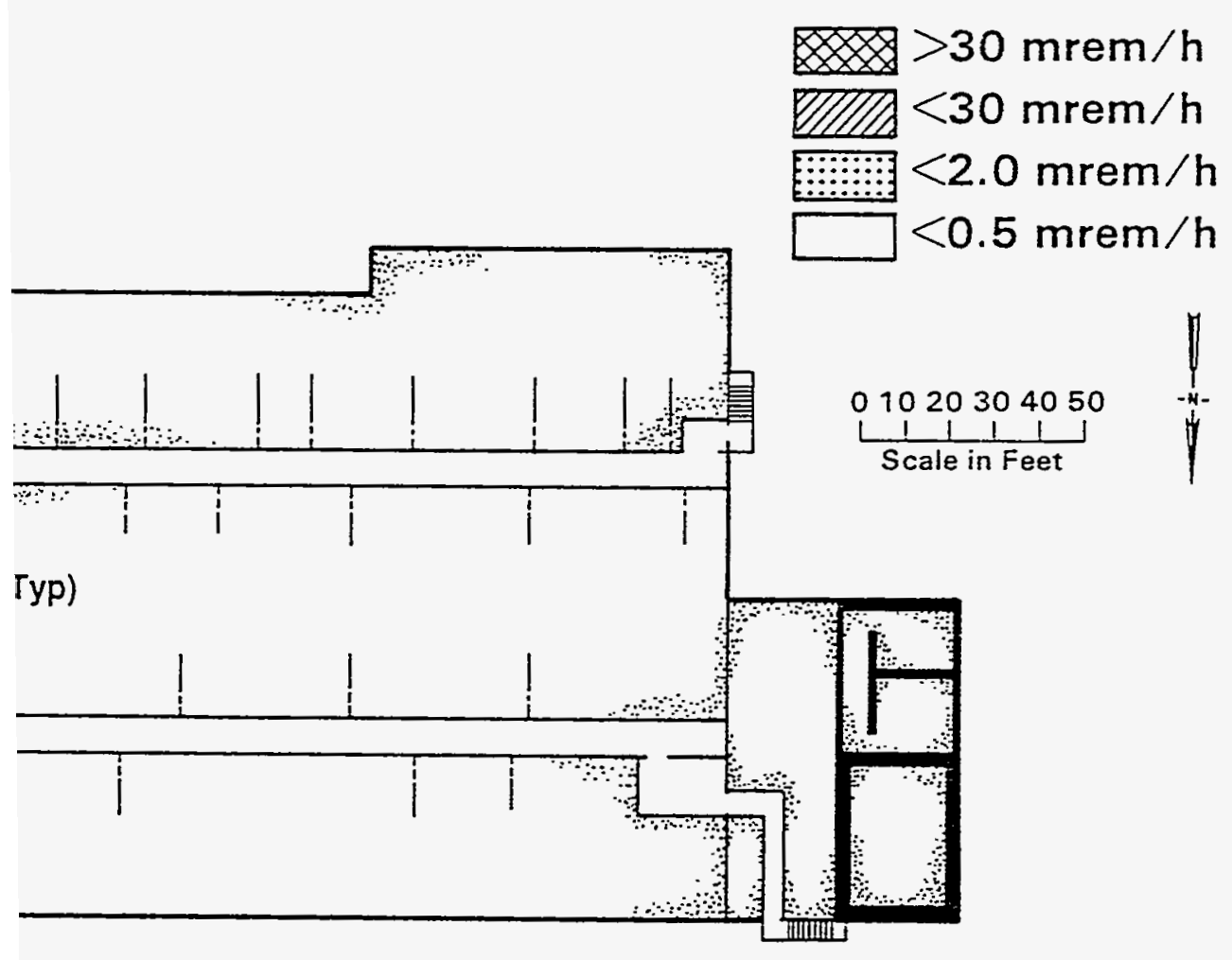

\section{el Plan}




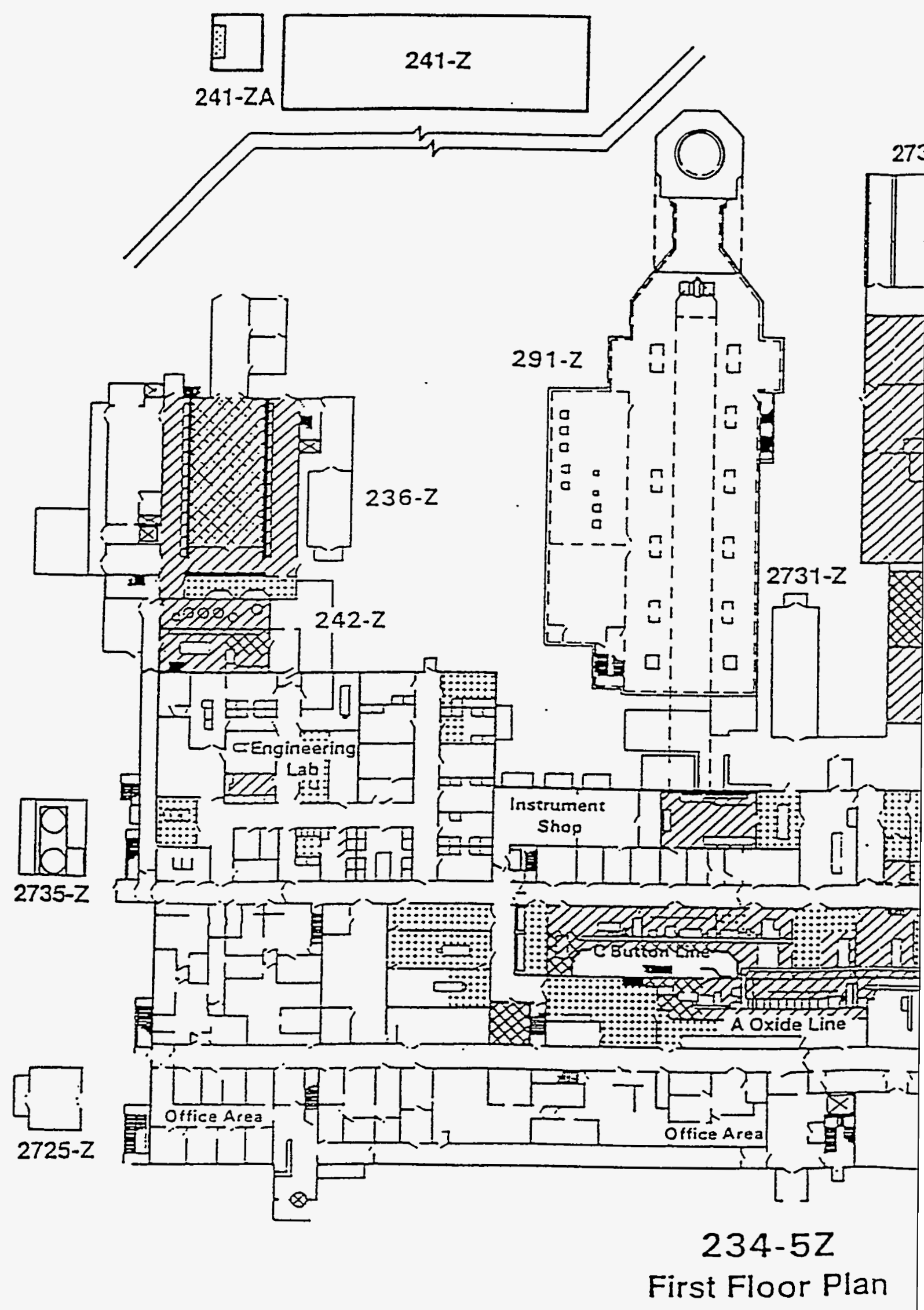


Figure 4-2. Plutonium Finishing Plant Radiation Dose Levels. (sheet 2 of 5)

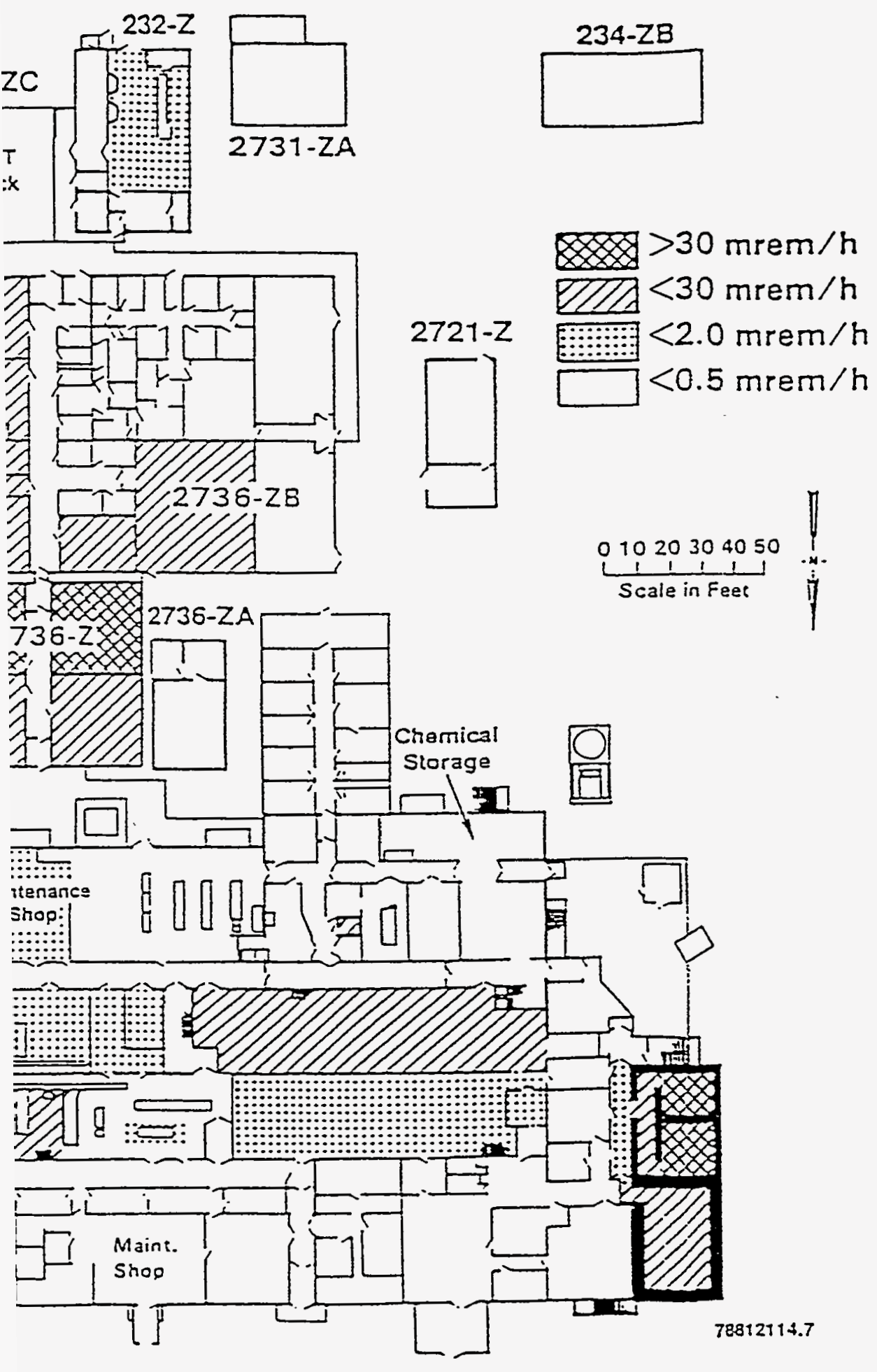




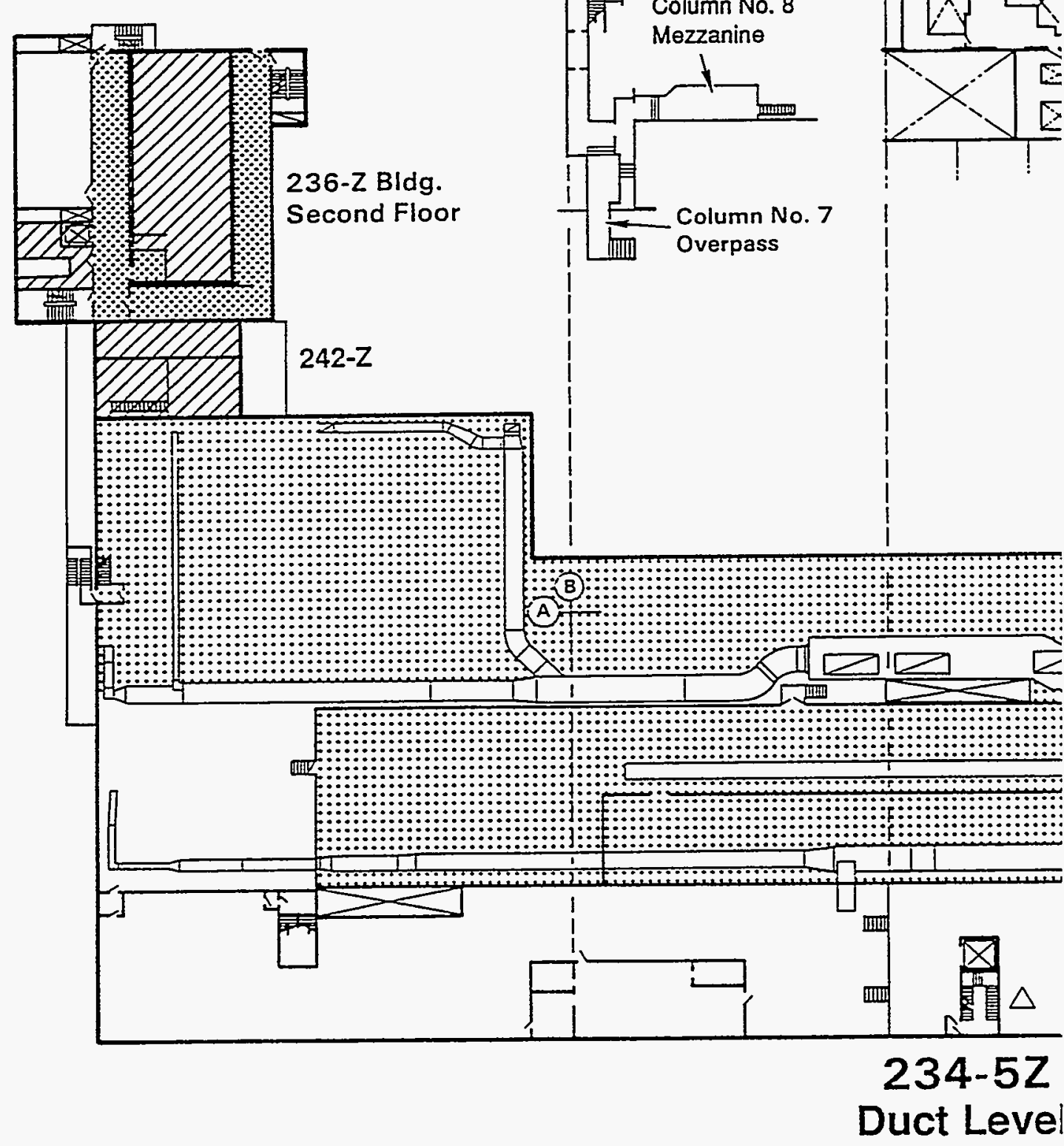


Figure 4-2. Plutonium Finishing Plant Radiation Dose Levels. (sheet 3 of 5 )

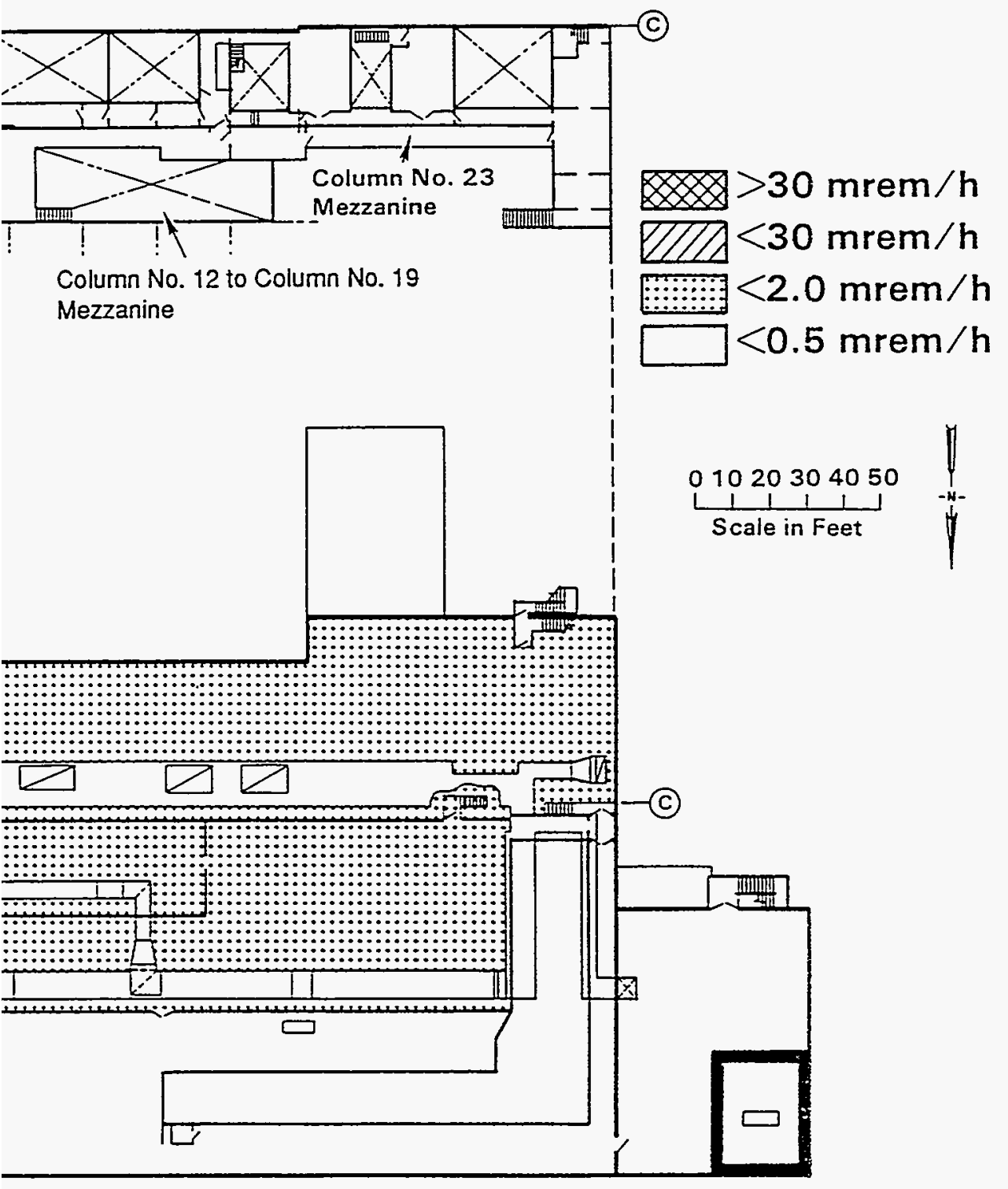

78812114.10 


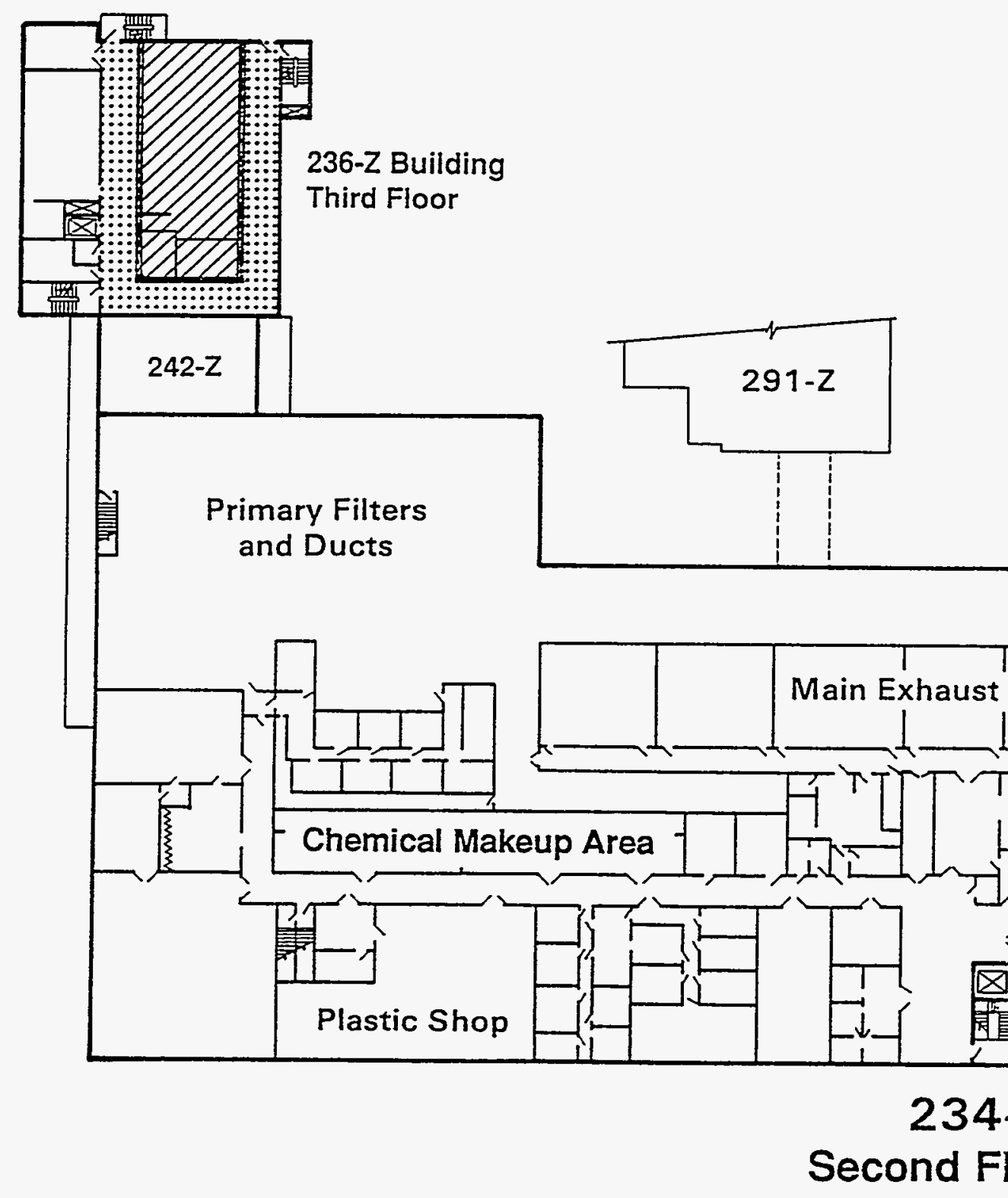


$\because$

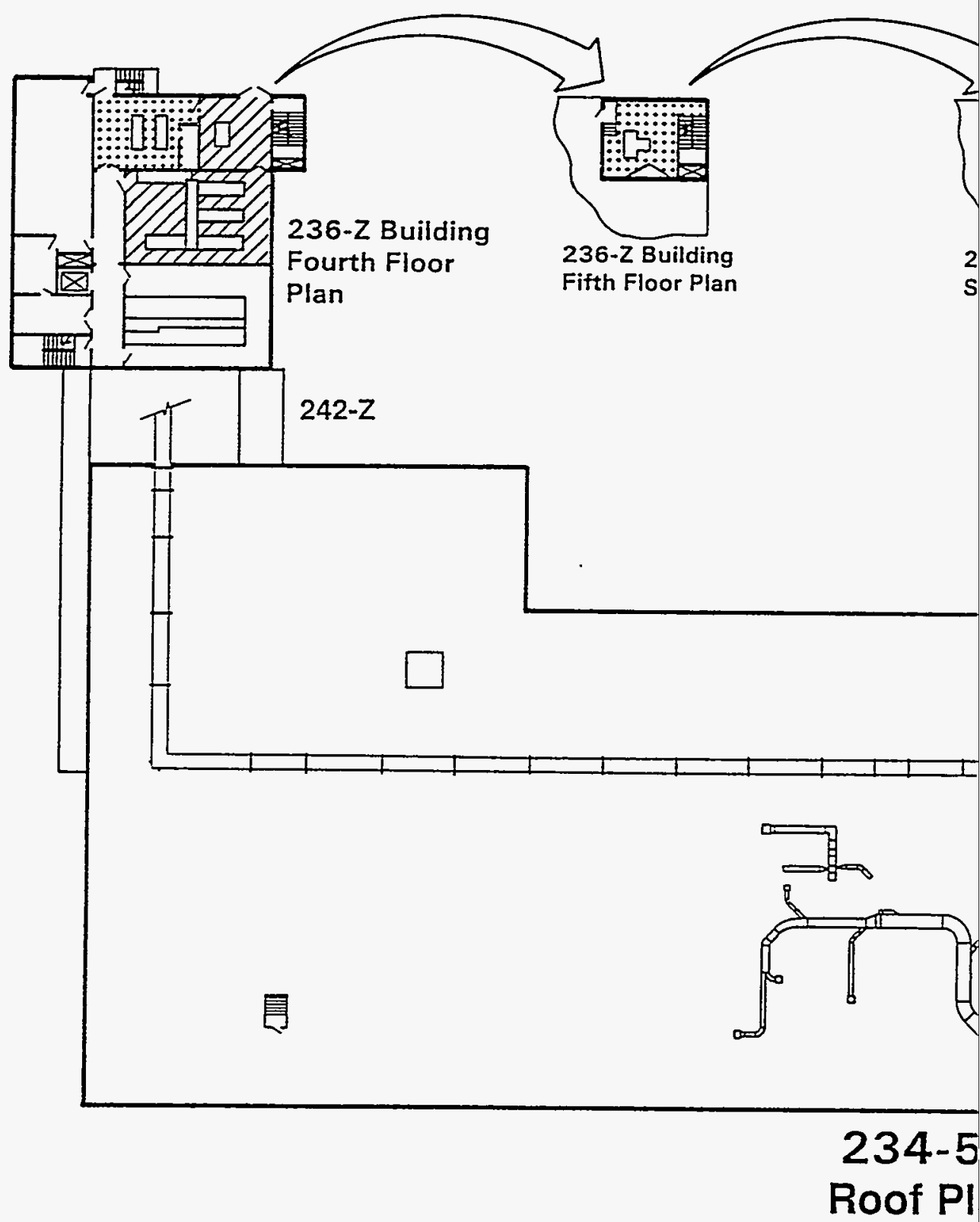


Figure 4-2. Plutonium Finishing Plant Radiation Dose Levels. (sheet 5 of 5 )

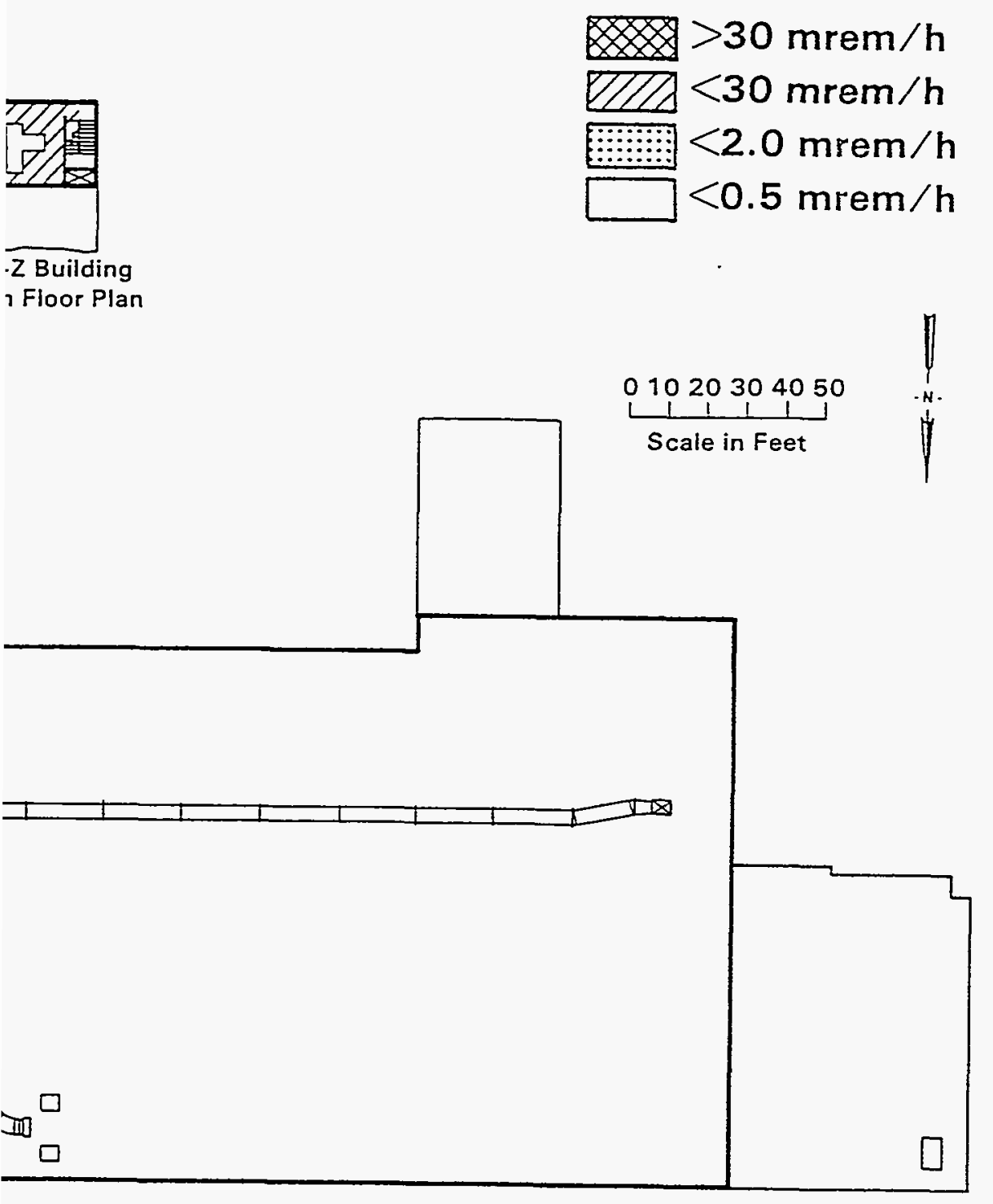

78812114.9 

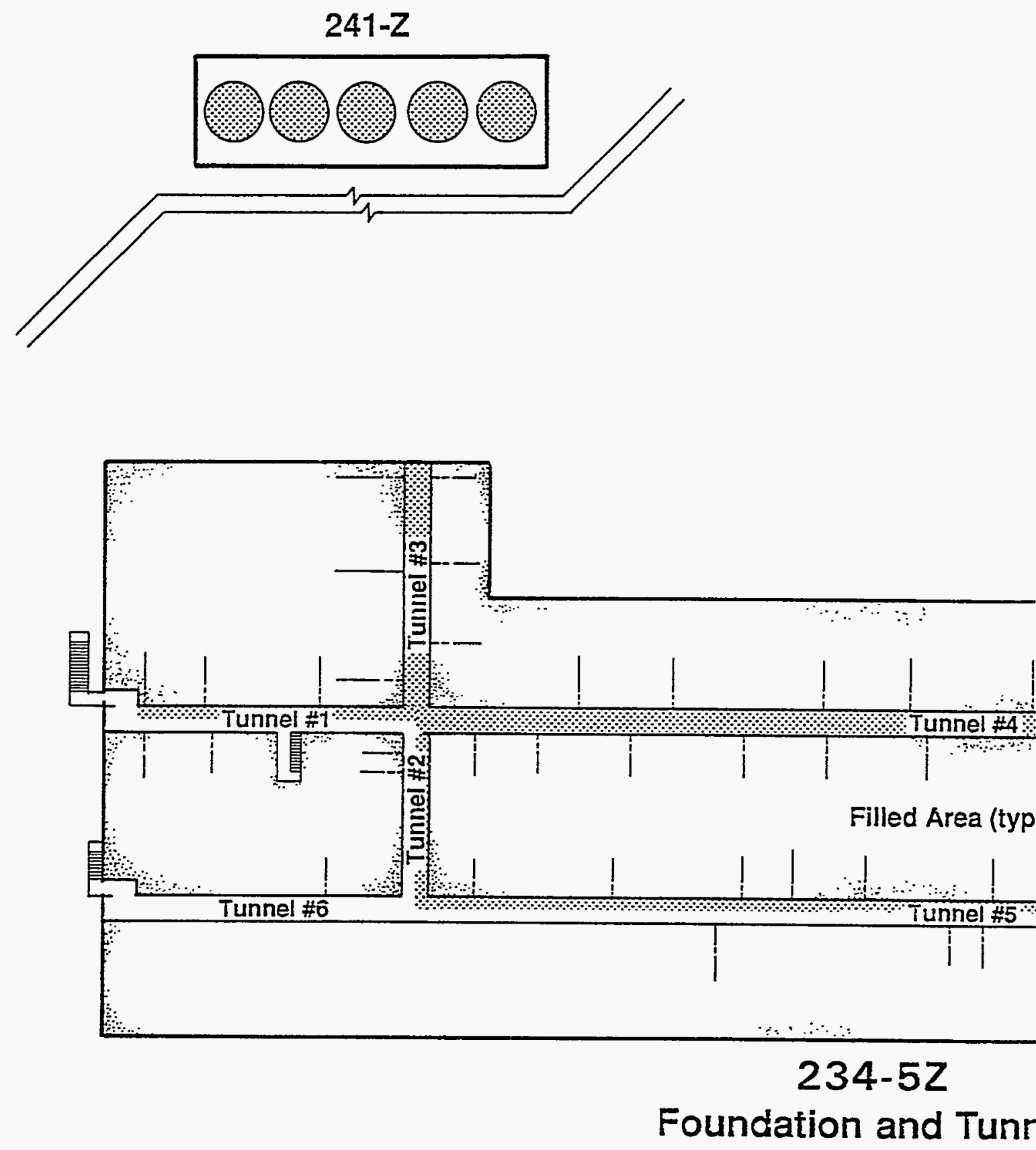
Figure 4-3. Location of Radiation Sources in the Plutonium Finishing Plant. (sheet 1 of 5)

\section{Location of Radiation} Sources

$\frac{\mid}{\text { Scale in Feet }}$

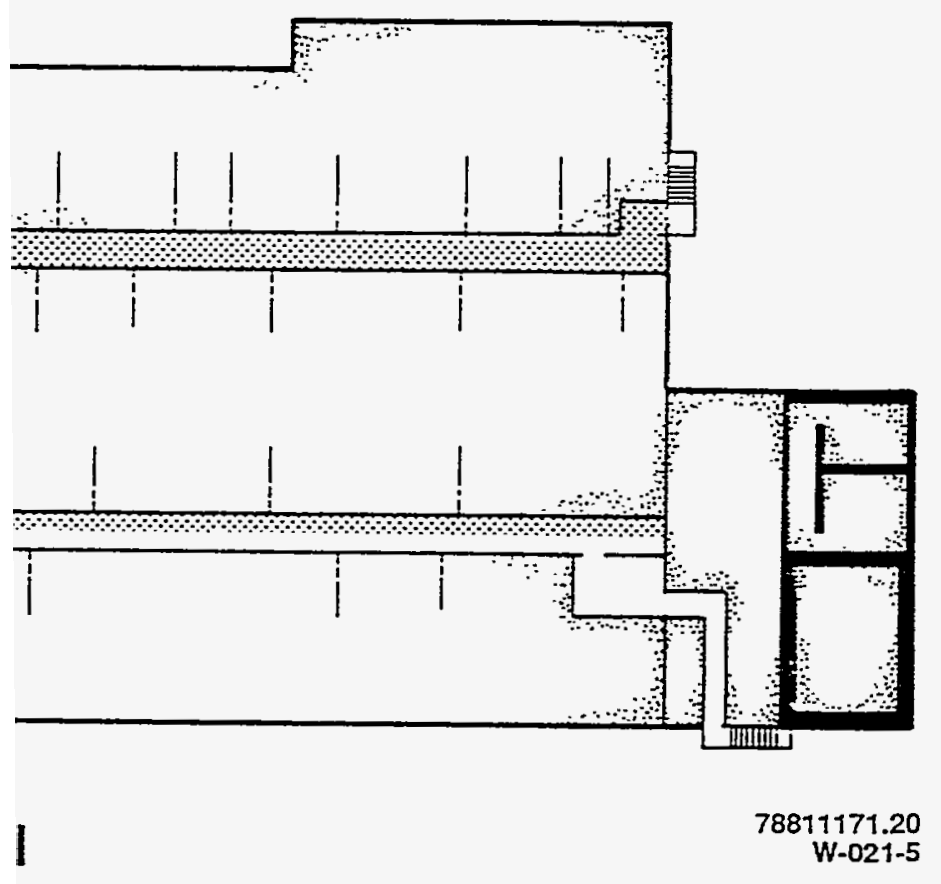




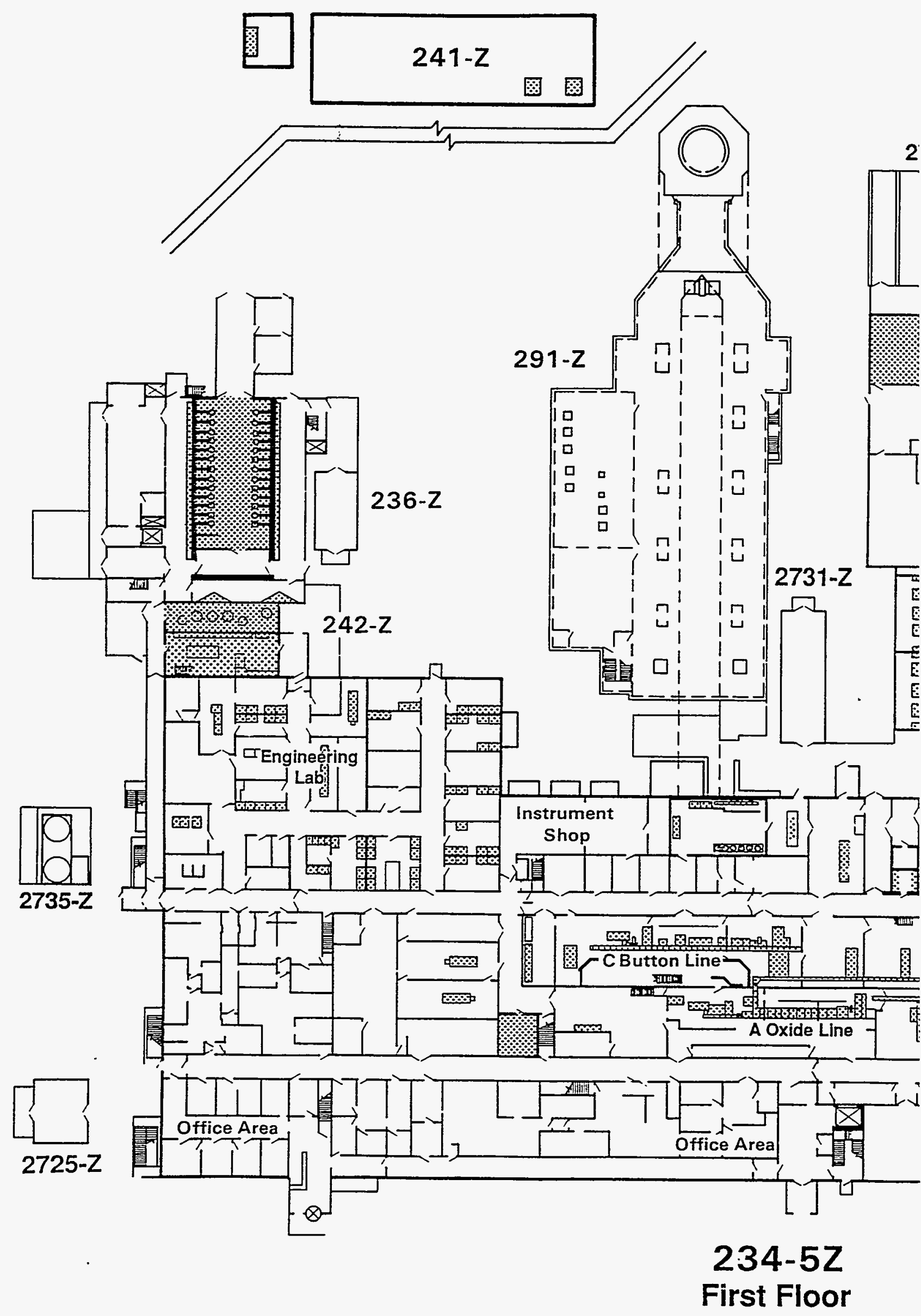




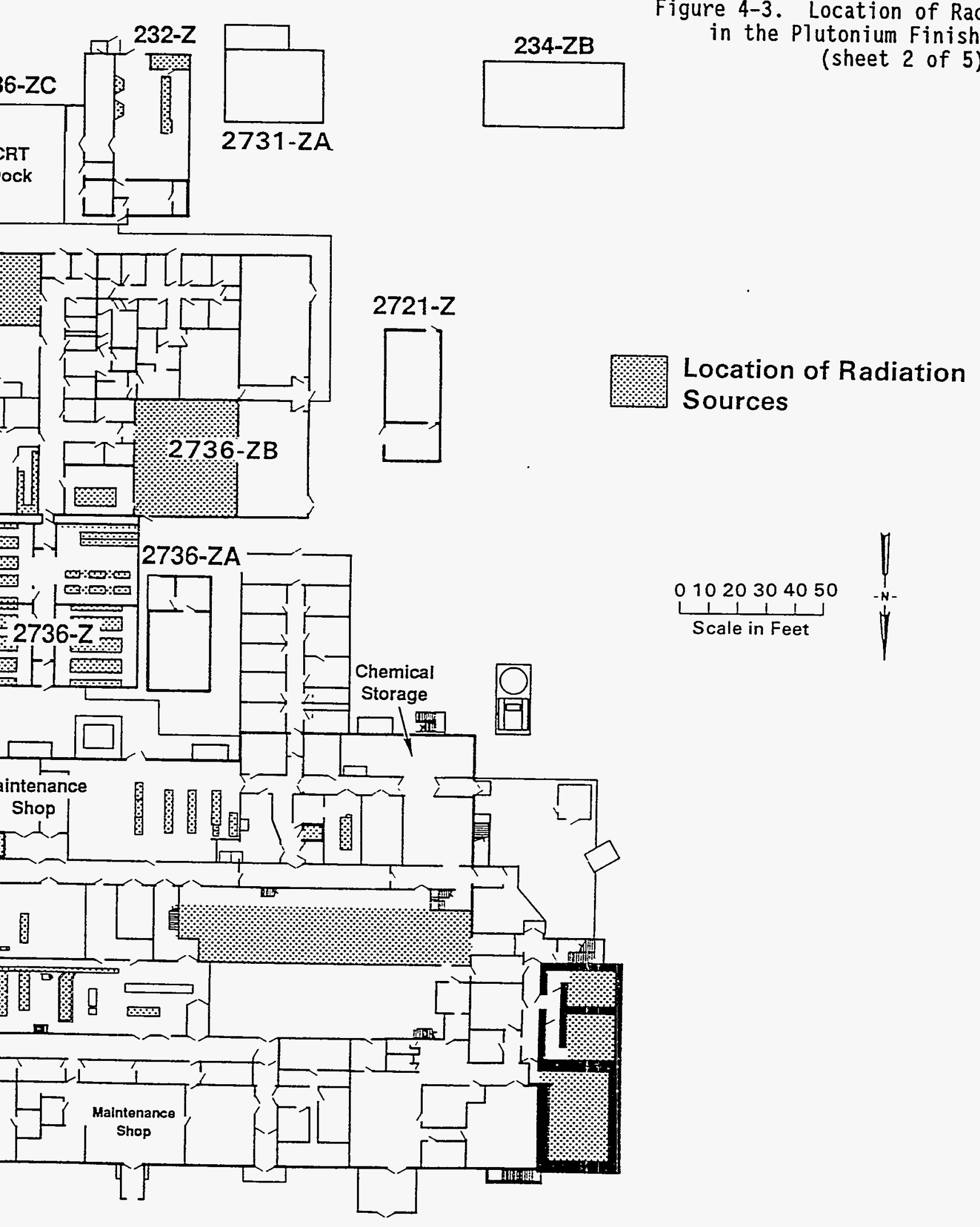




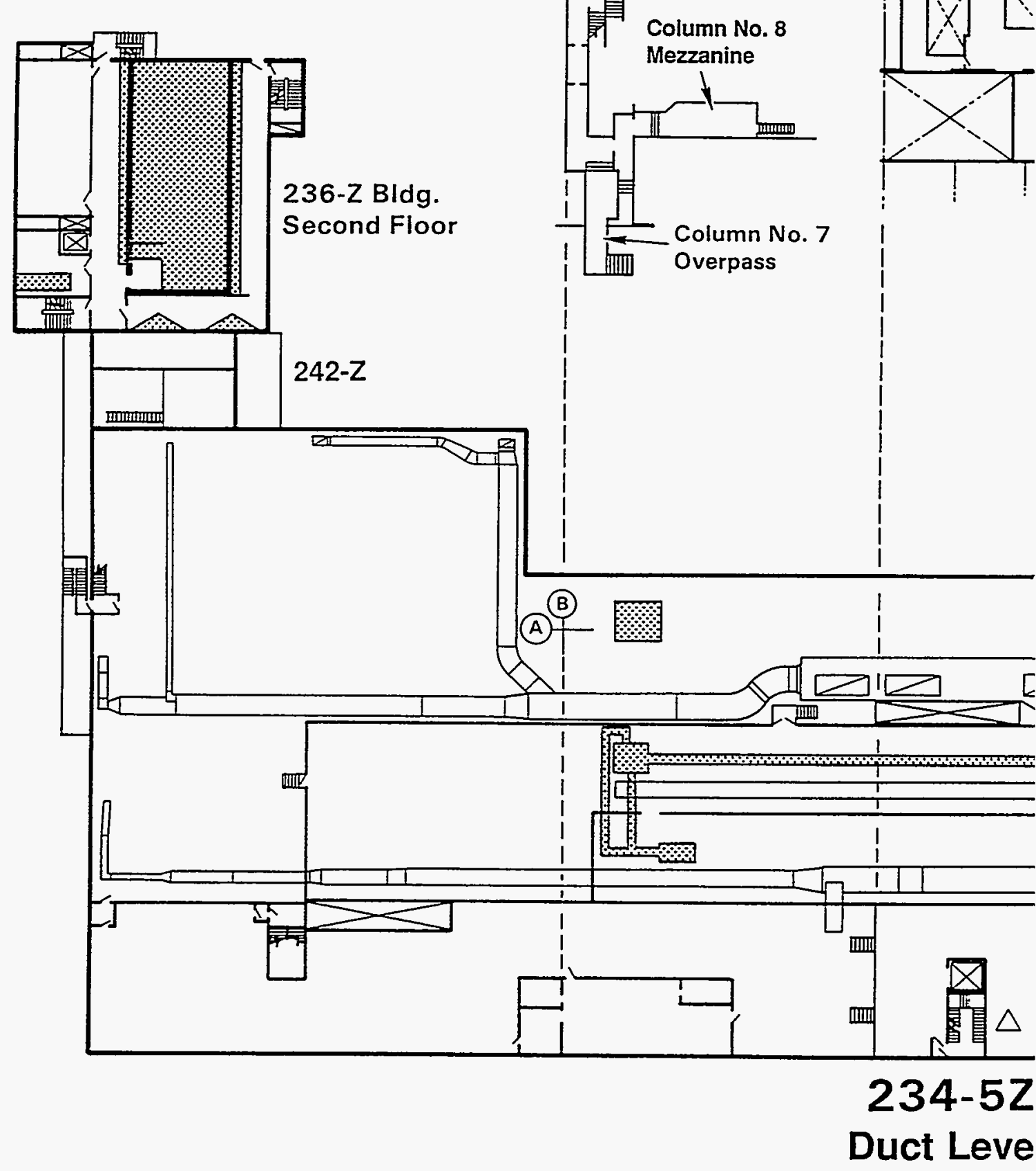




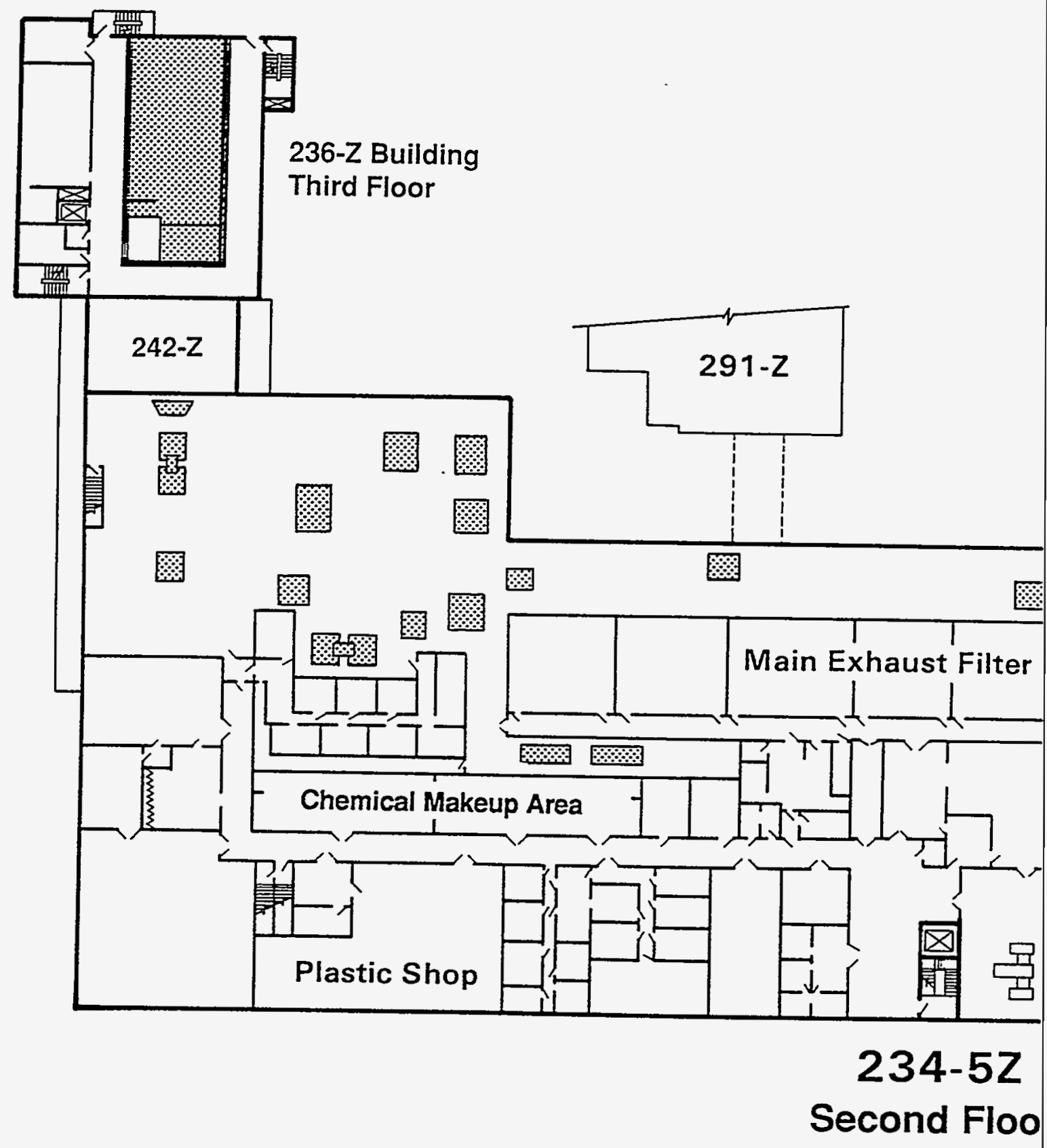


Figure 4-3. Location of Radiation Sources in the Plutonium Finishing Plant.

(sheet 4 of 5)

\section{Location of Radiation} Sources

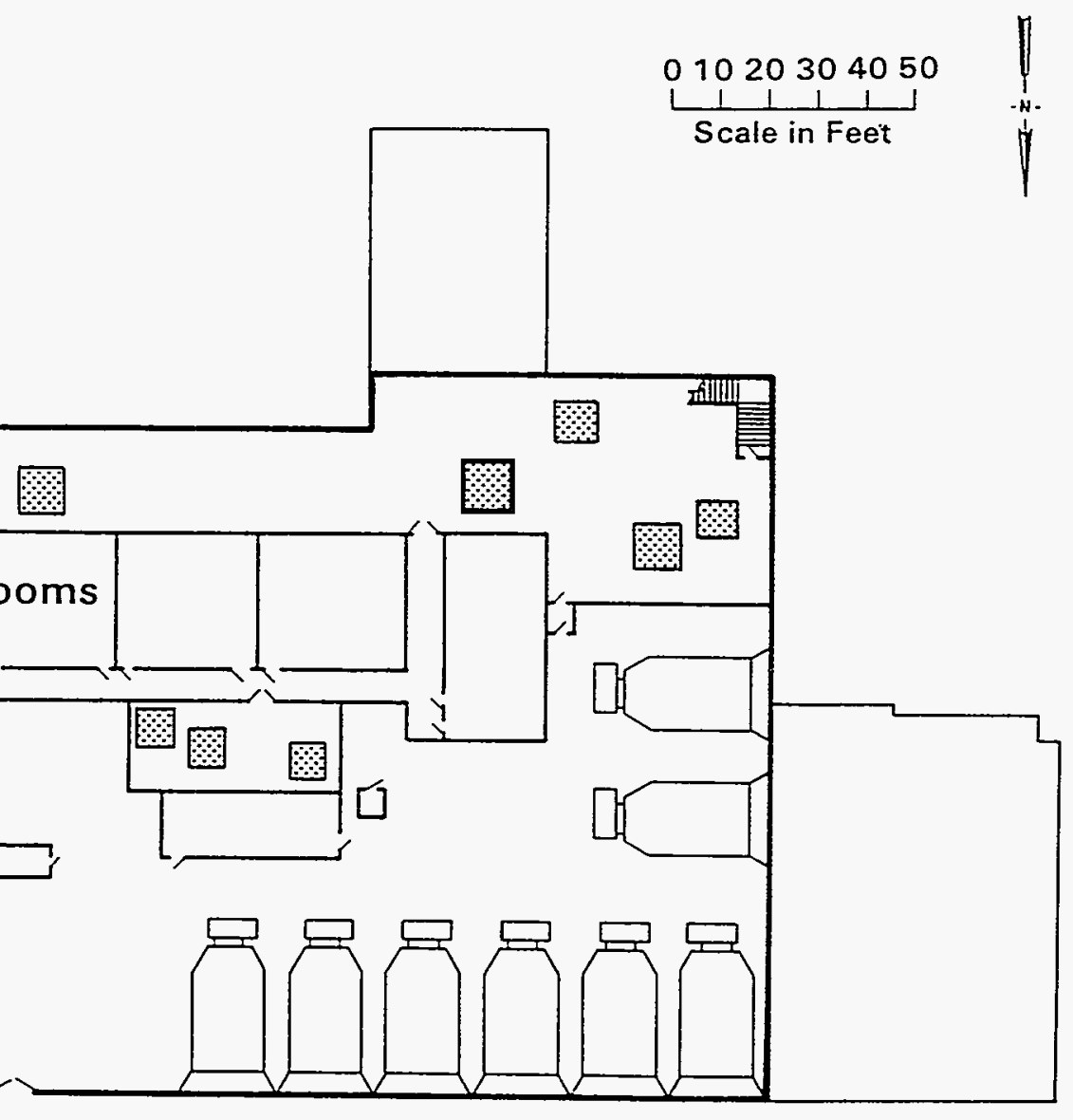

78811171.18 


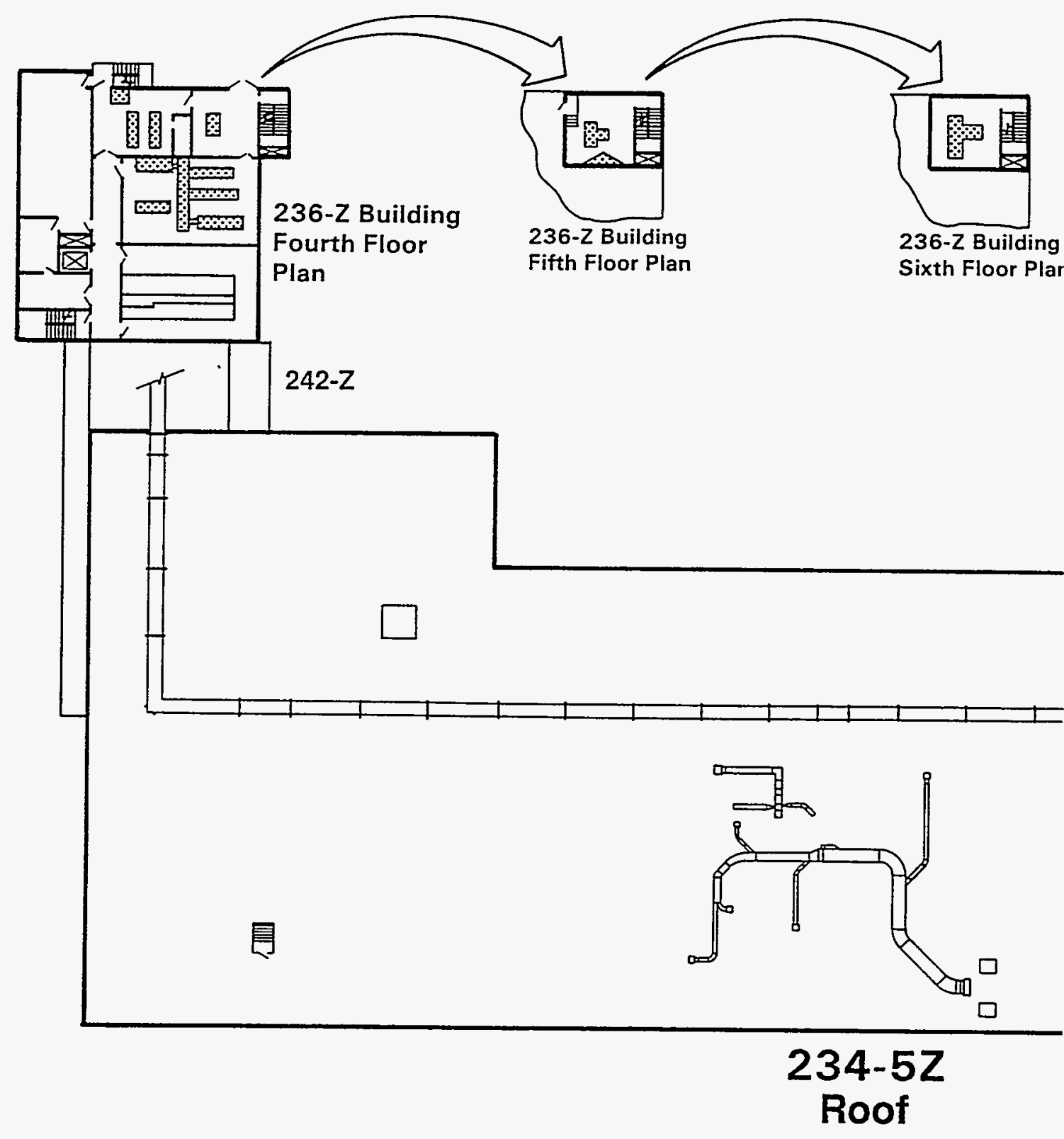


Figure 4-3. Location of Radiation Sources in the Plutonium Finishing Plant. (sheet 5 of 5)

\section{Location of Radiation} Sources

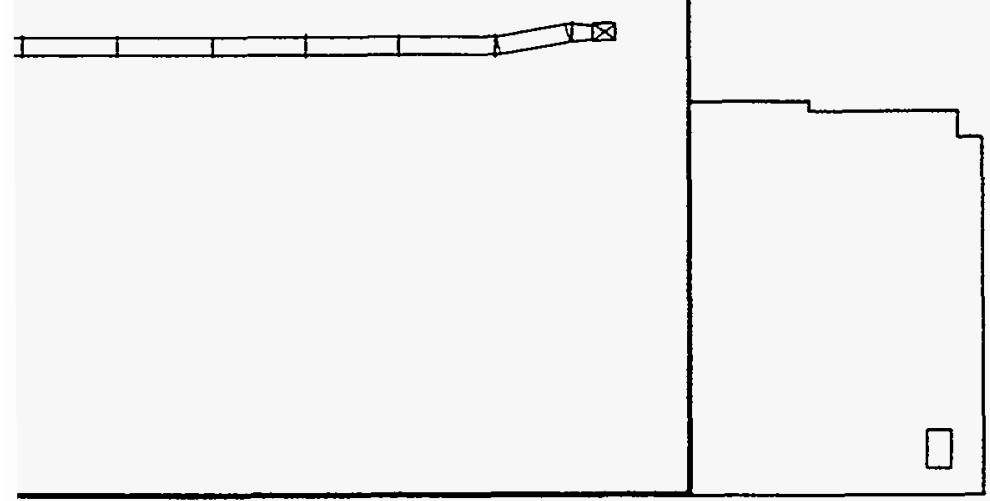

78812114.58 
- The room has a glovebox located within it according to Figure 9.0.2-3 or 9.0.2-4 in WHC-SD-CP-SAR-021 (WHC 1991) (see Figure 4-4).

- The room is classified as a contamination control area according to Figure 8.3-11 in WHC-SD-CP-SAR-021 (WHC 1991) (see Figure 4-5).

- The room is posted as an airborne radioactivity area or a surface contamination area as of January 1994 (see Figure 4-6).

"Cold" process support areas are defined as rooms that are not "hot" process areas, yet any of the following conditions are met.

- The room is in a radiation area according to Figure 8.3-8 in WHC-SD-CP-SAR-021 (HHC 1991) (see Figure 4-1).

- The room has a normal dose level reading of greater than $0.5 \mathrm{mrem} / \mathrm{h}$ but less than $2.0 \mathrm{mrem} / \mathrm{h}$ according to Table 8.4-1 and Figure 8.3-17 in WHC-SD-CP-SAR-021 (WHC 1991) (see Figure 4-2).

- The room is classified as a controlled access area according to Figure 8.3-11 in WHC-SD-CP-SAR-021 (WHC 1991) (see Figure 4-5).

- The room is posted as a radiological control area as of January 1994 (see Figure 4-6).

NOTE: Uncontaminated areas are all other rooms and will not be considered during this study.

A spreadsheet was created to correlate relevant sources of information and classify the facility by contamination level: building by building, room by room. Sources included Table 8.4-1 and Figures 8.3-7, 8.3-8, 8.3-11, 8.3-17, and 9.0.2-3 from WHC-SD-CP-SAR-021 (WHC 1991) (included in this report as Table 4-1 and Figures 4-1 through 4-6), and the radiological posting for PFP as of January 1994. The classification used for this study is detailed in Table 4-2 and illustrated in Figure 4-7. Complete information used to compile this classification can be found in Appendix $A$.

Next, a document review was performed. By reviewing all of the Criticality Prevention Specifications for the PFP (WHC 1994a), relevant drawings were found for both "hot" process areas and "cold" process support areas. The reference criticality prevention specifications and relevant drawings were linked to the rooms in the spreadsheet (see Appendix A). From the documentation and drawing information available, a room by room list of contaminated solid waste items and their dimensions was prepared and entered into the spreadsheet, sorted into the following natural physical categories:

- Gloveboxes and hoods (i.e., tanks, process piping, manifolds, and other items contained by the gloveboxes).

- Tanks not in a glovebox (i.e., PRF canyon pencil tanks and columns, tanks in the chemical preparation area, 241-Z waste tanks, and the caustic storage tank). 
WHC-EP-0829

This page intentionally left blank. 


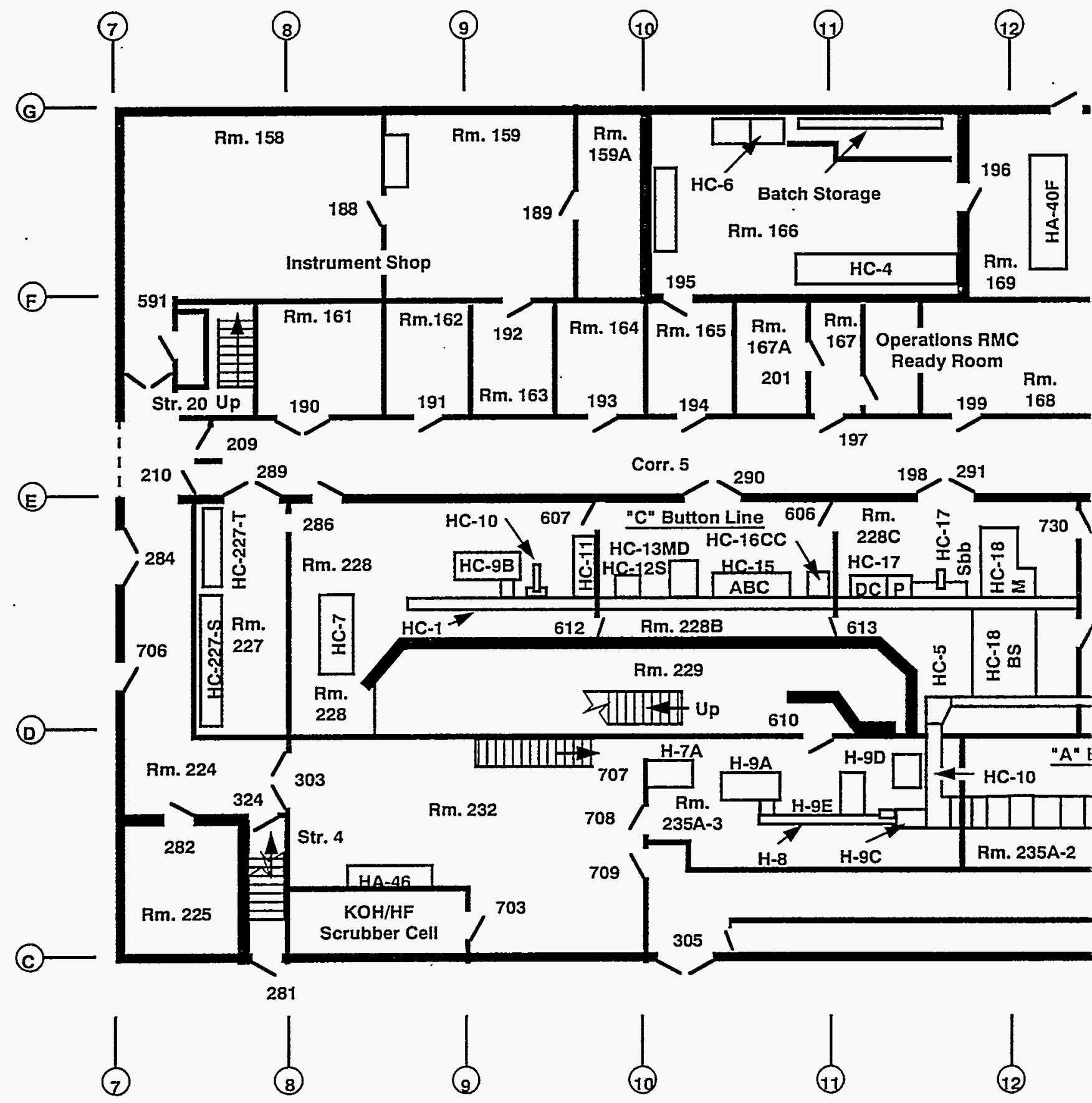


Figure 4-4. Location of Gloveboxes Within Buildings 234-5Z and 236-Z.

(sheet 1 of 2)

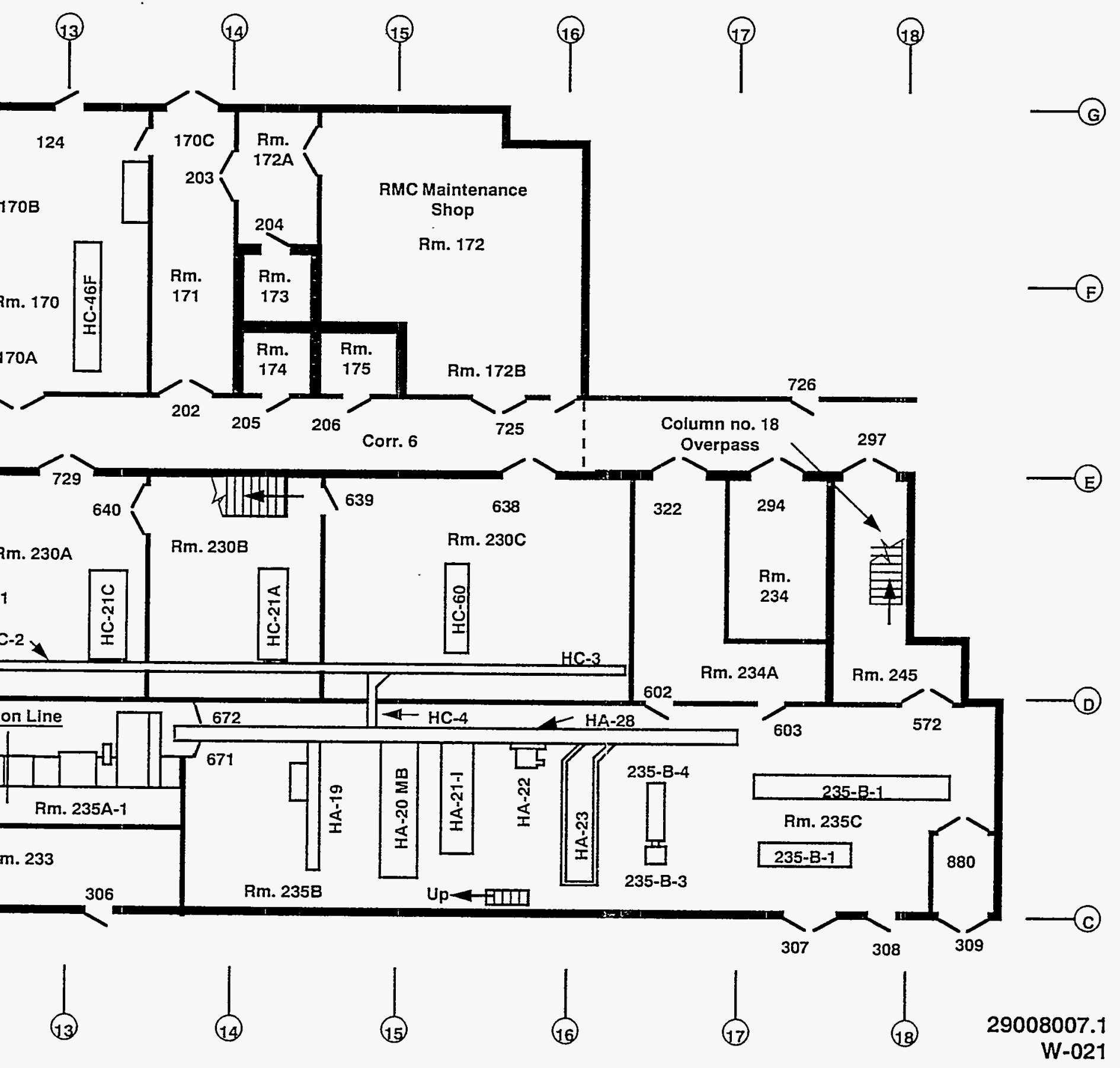




\section{Building 236-Z Floor Plans}
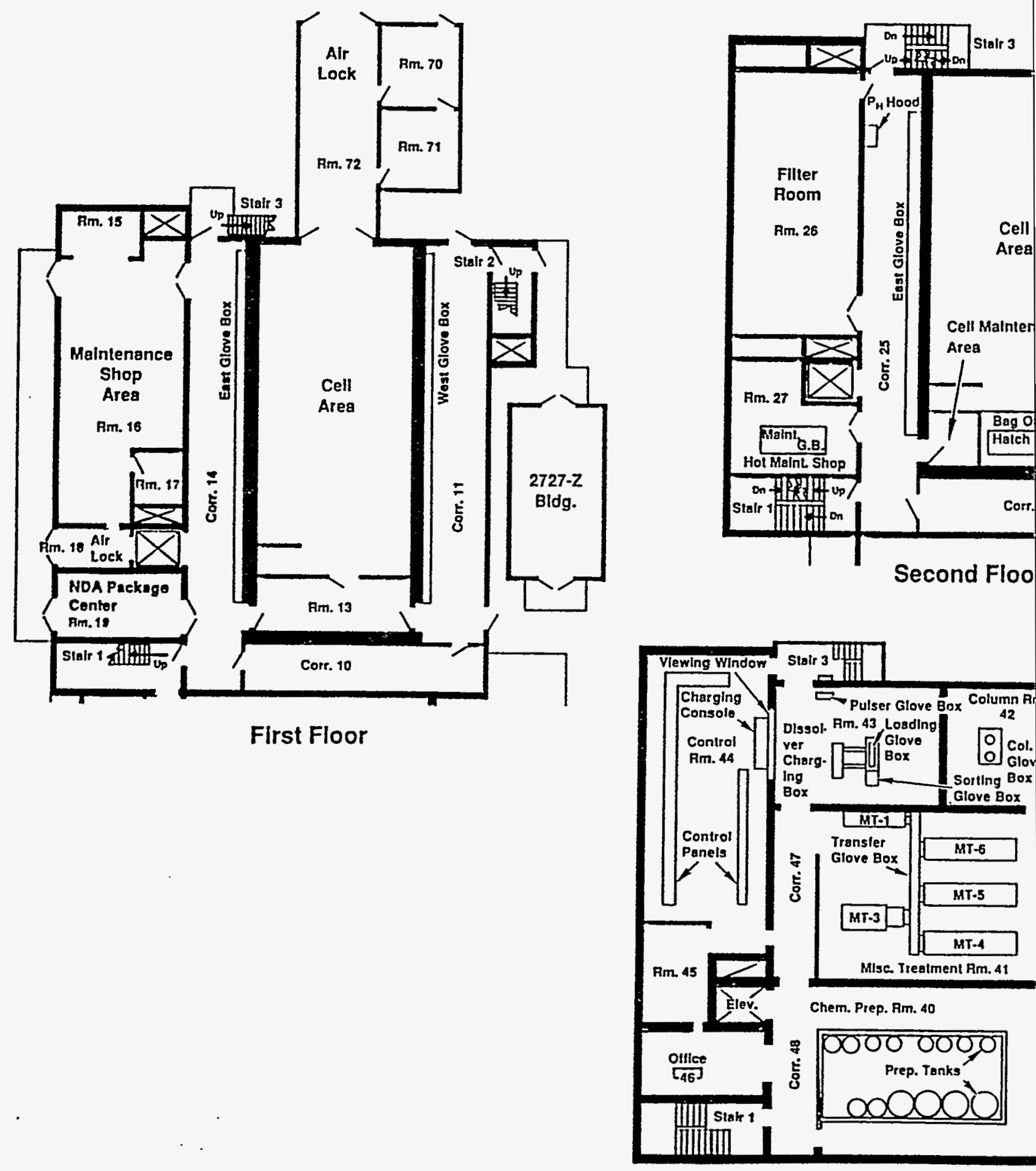

First Floor

Fourth Floor 


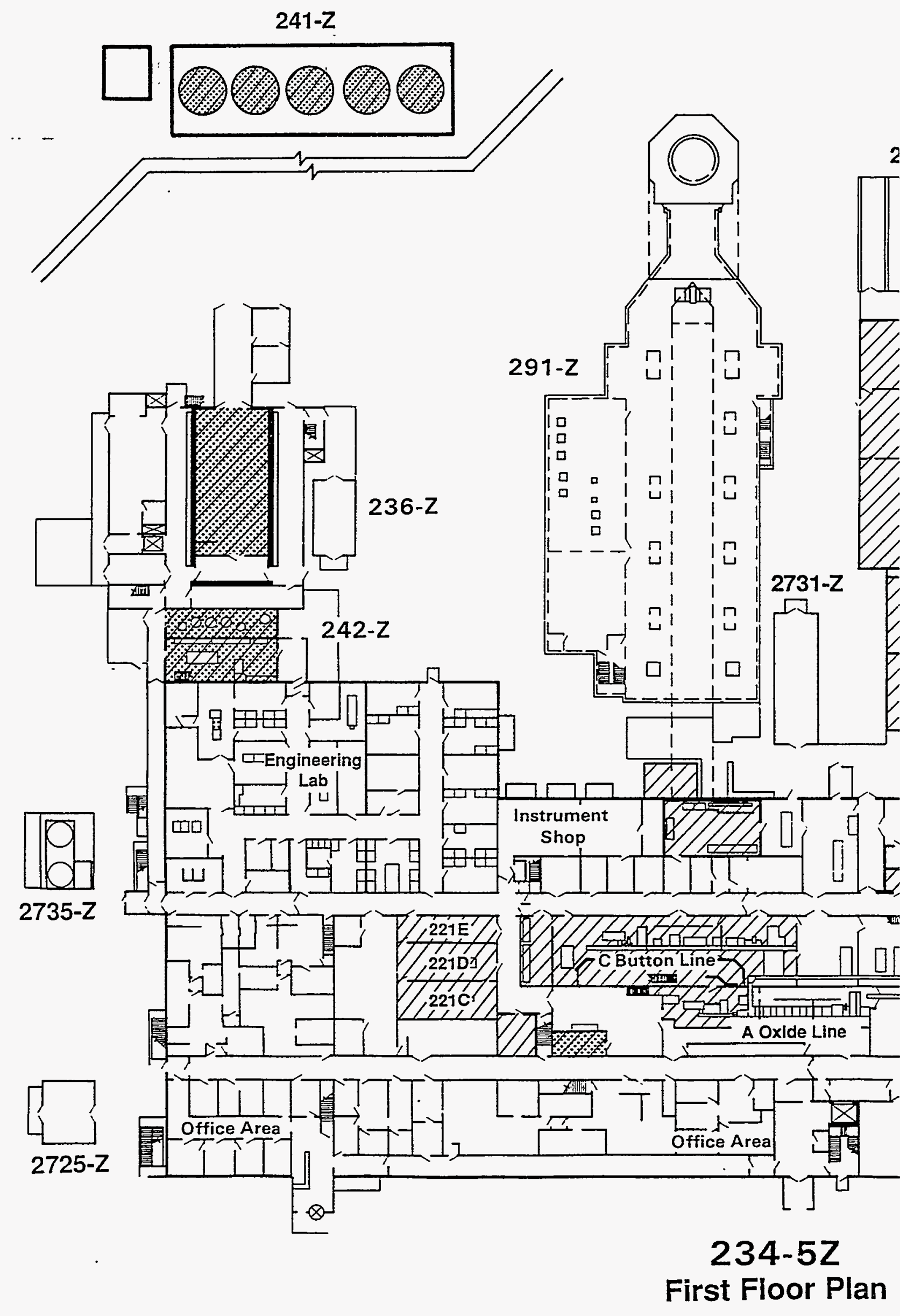




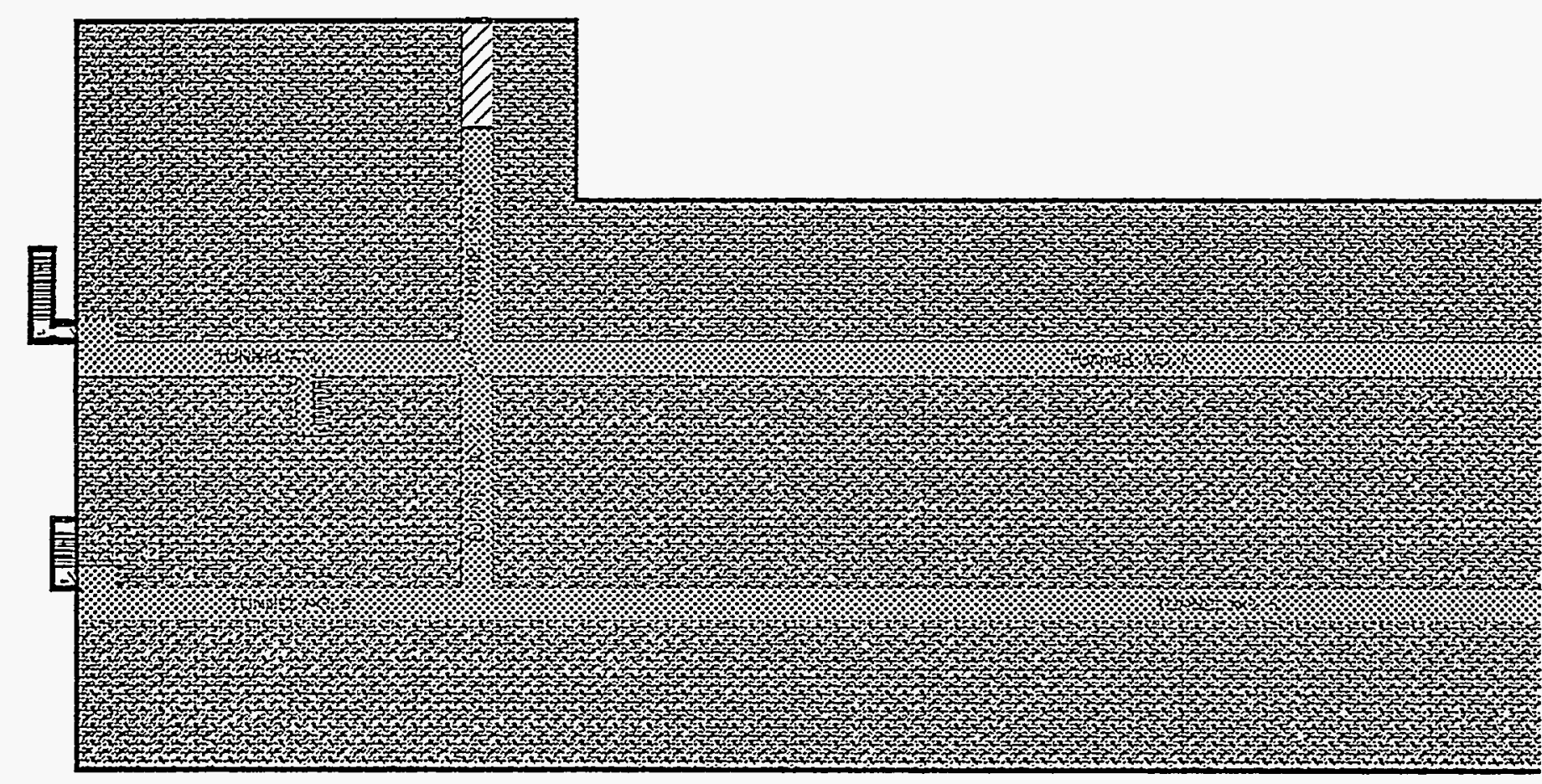




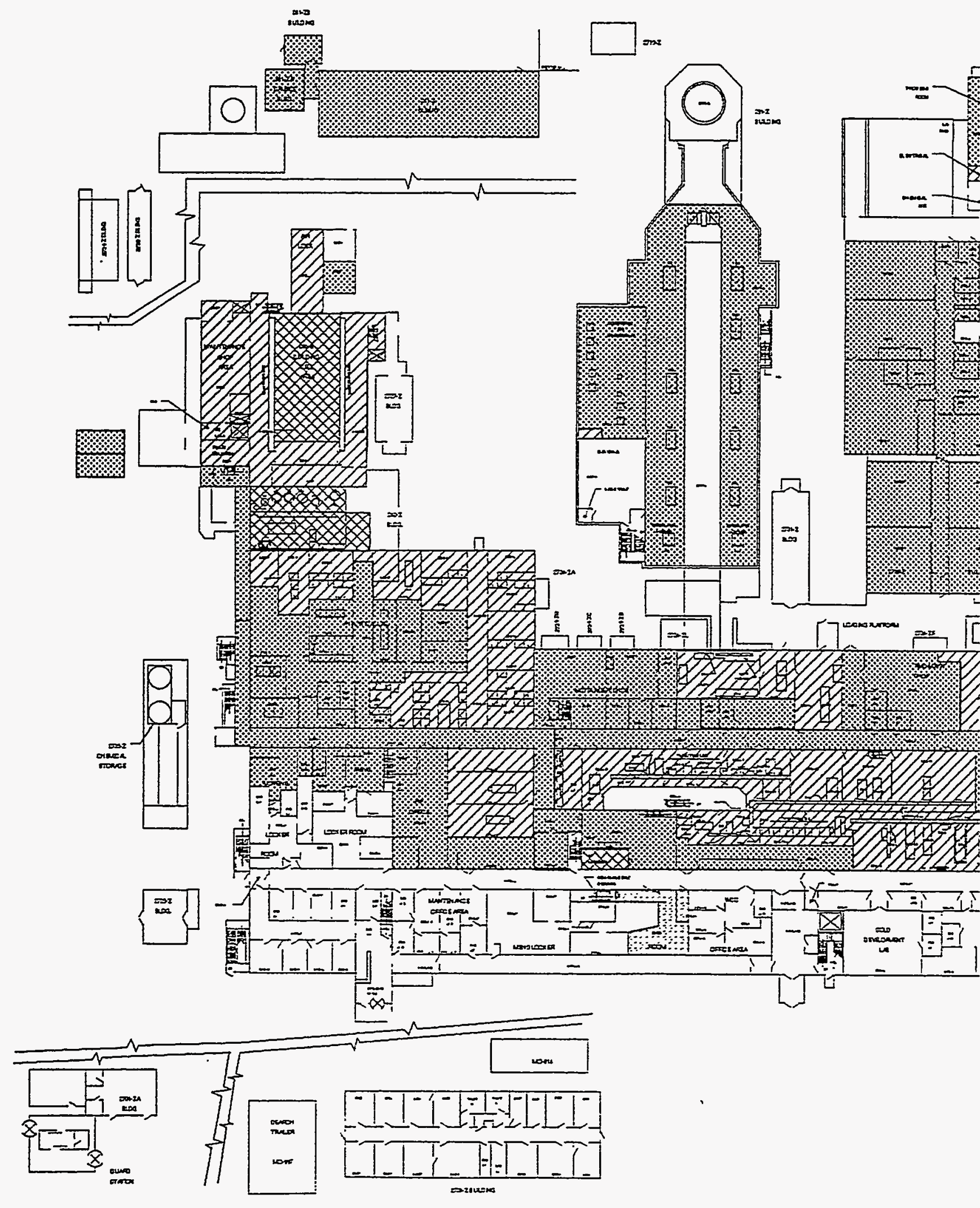




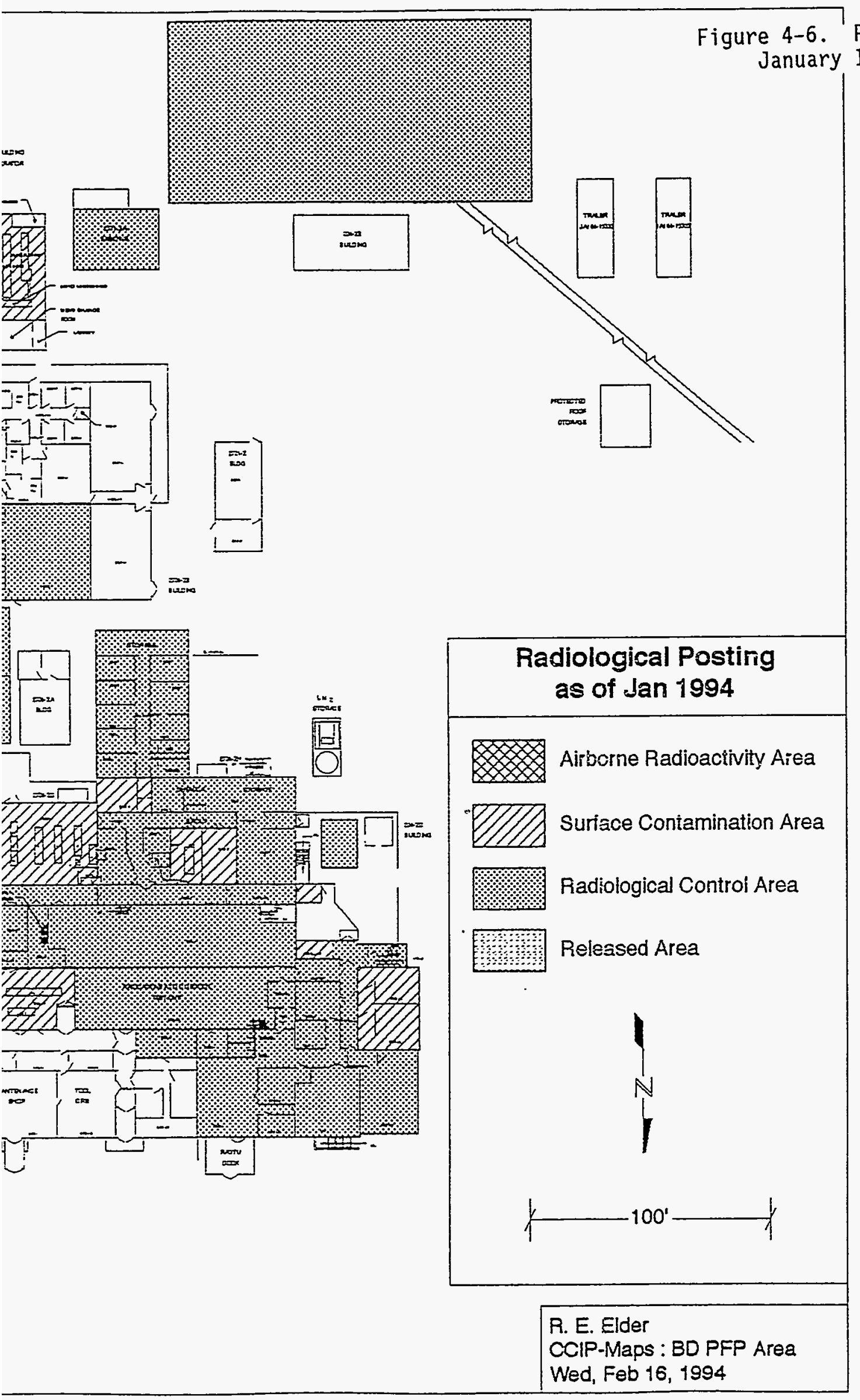




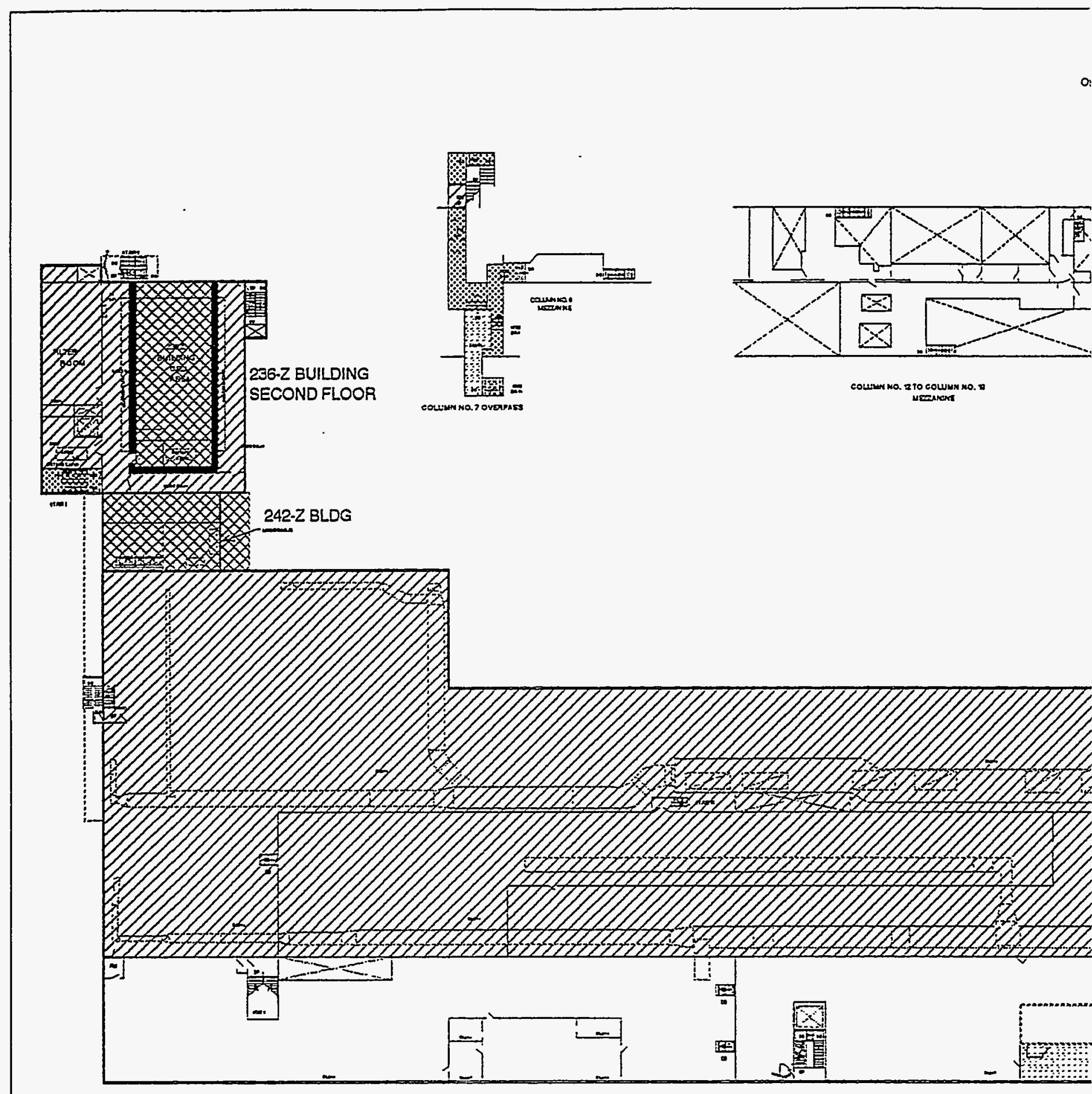




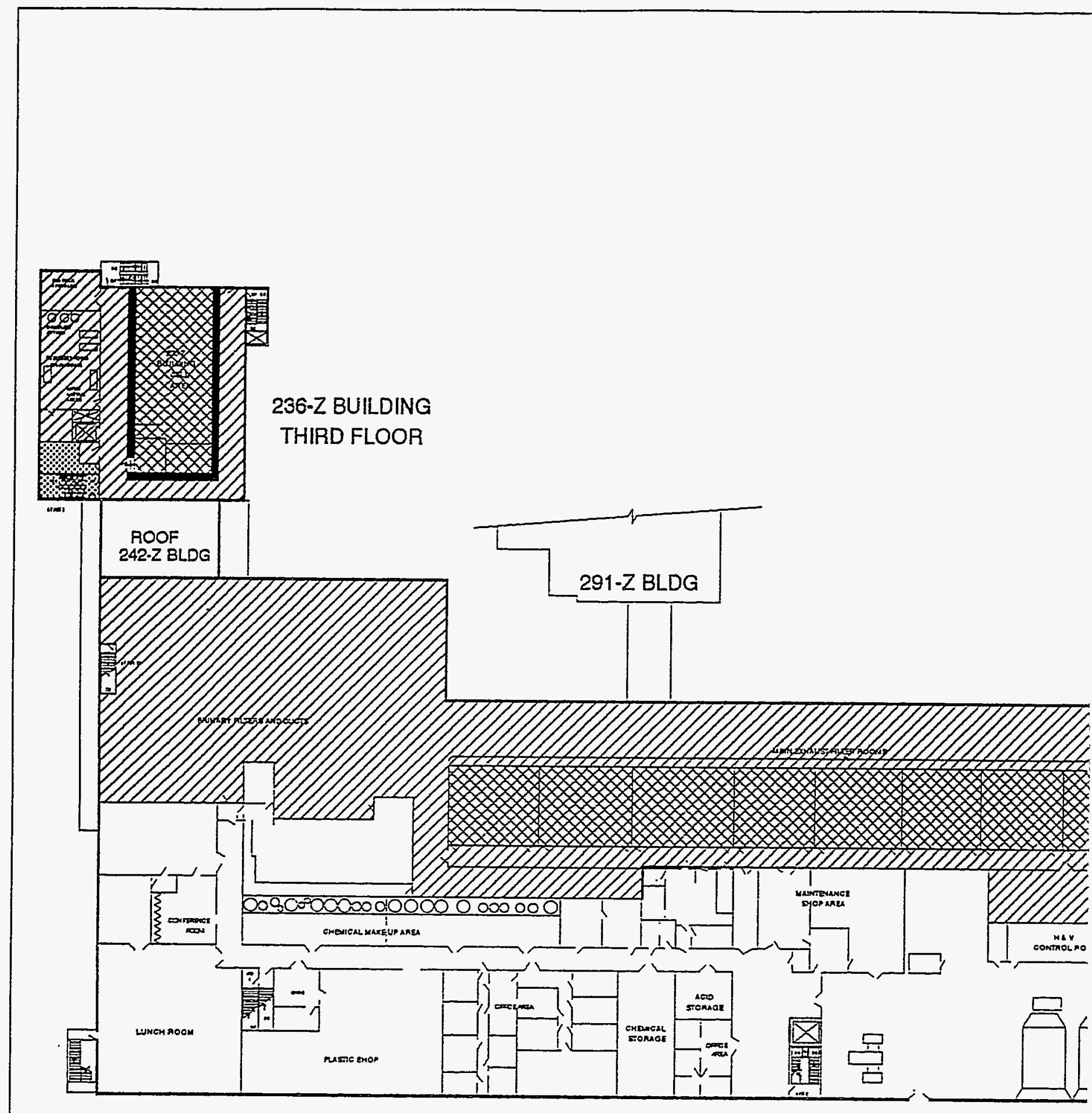


Figure 4-6. Radiological Posting as of January 1994. (sheet 4 of 5)
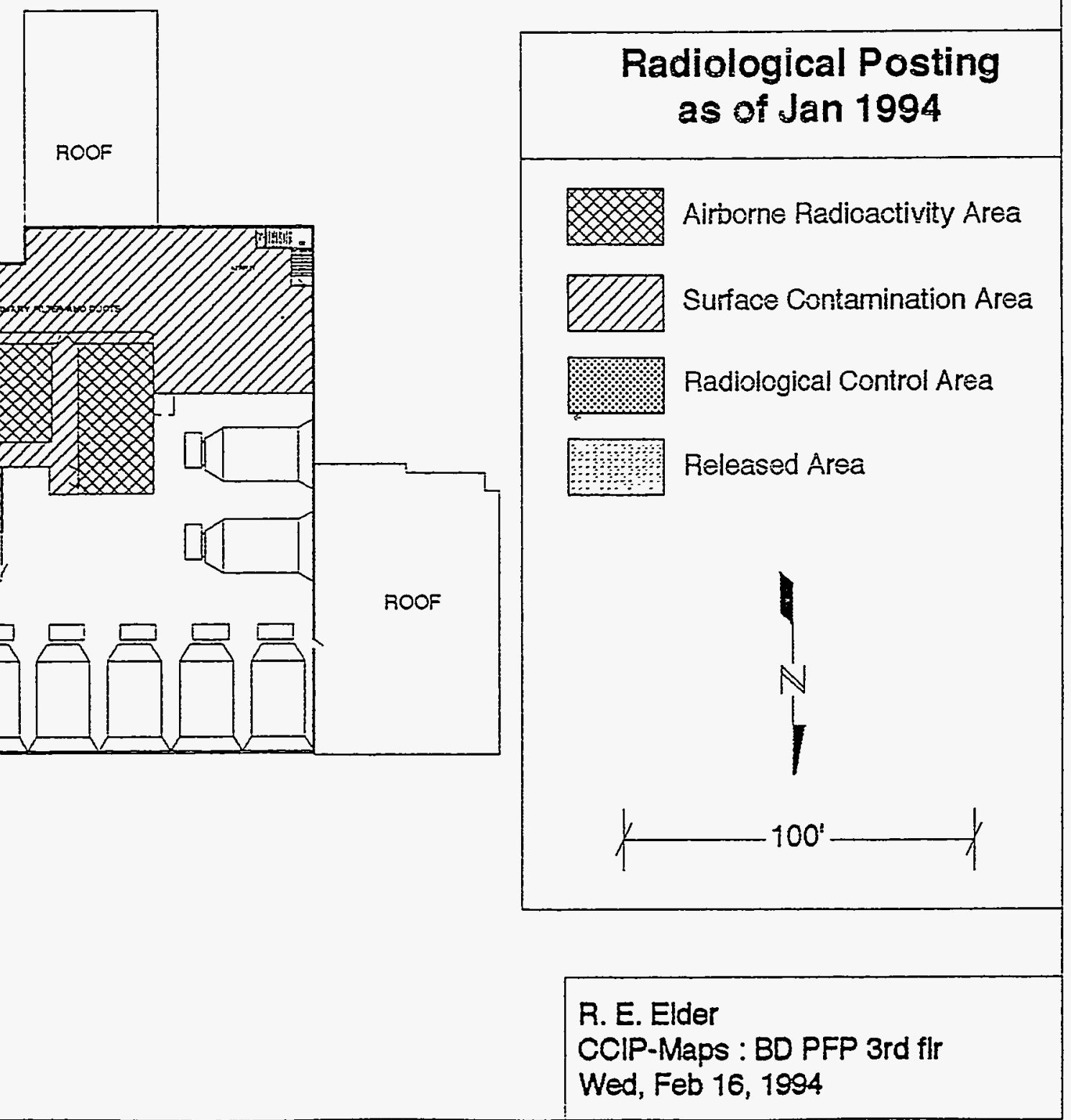


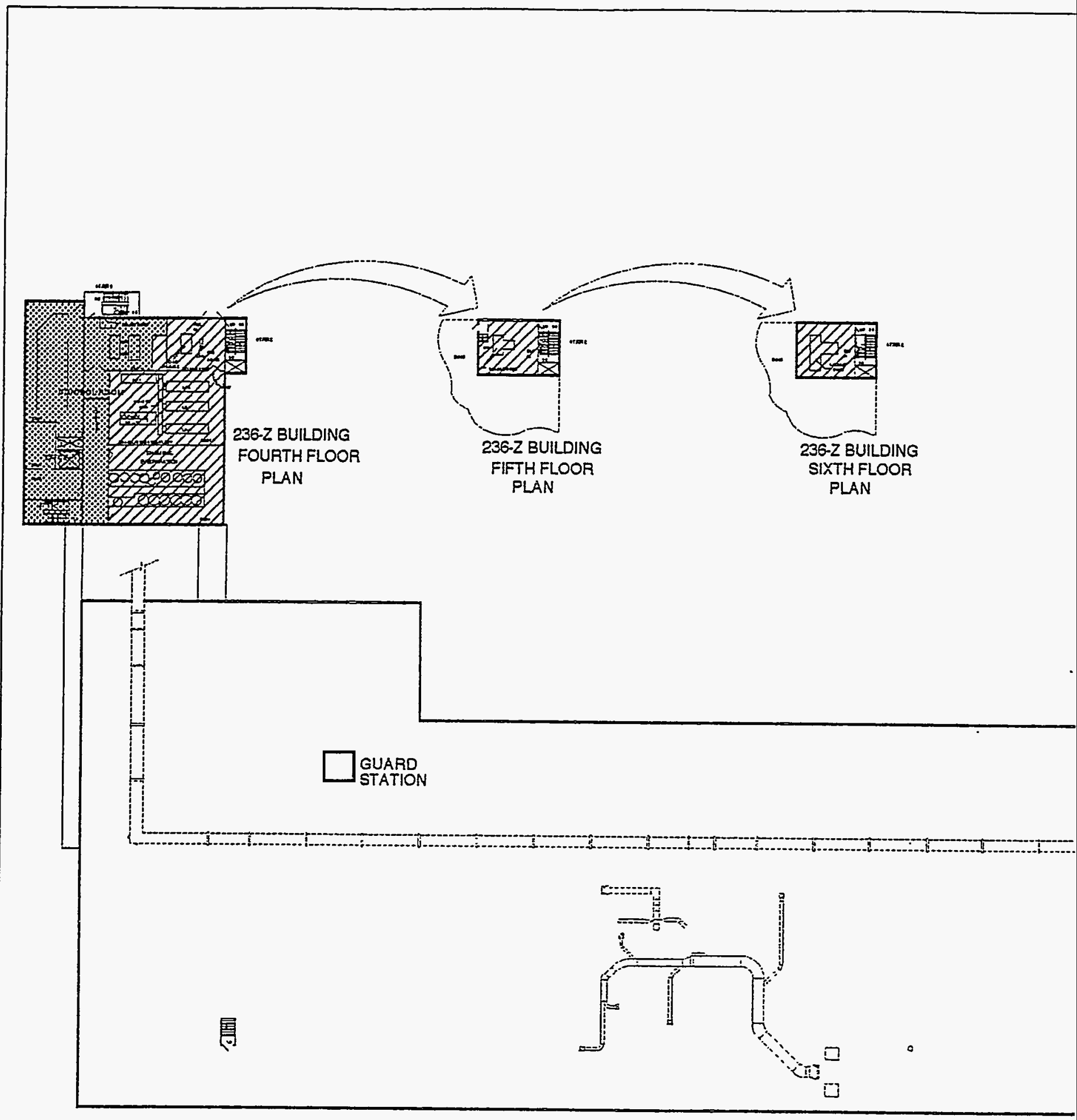


Figure 4-6. Radiological Posting as of January 1994. (sheet 5 of 5)

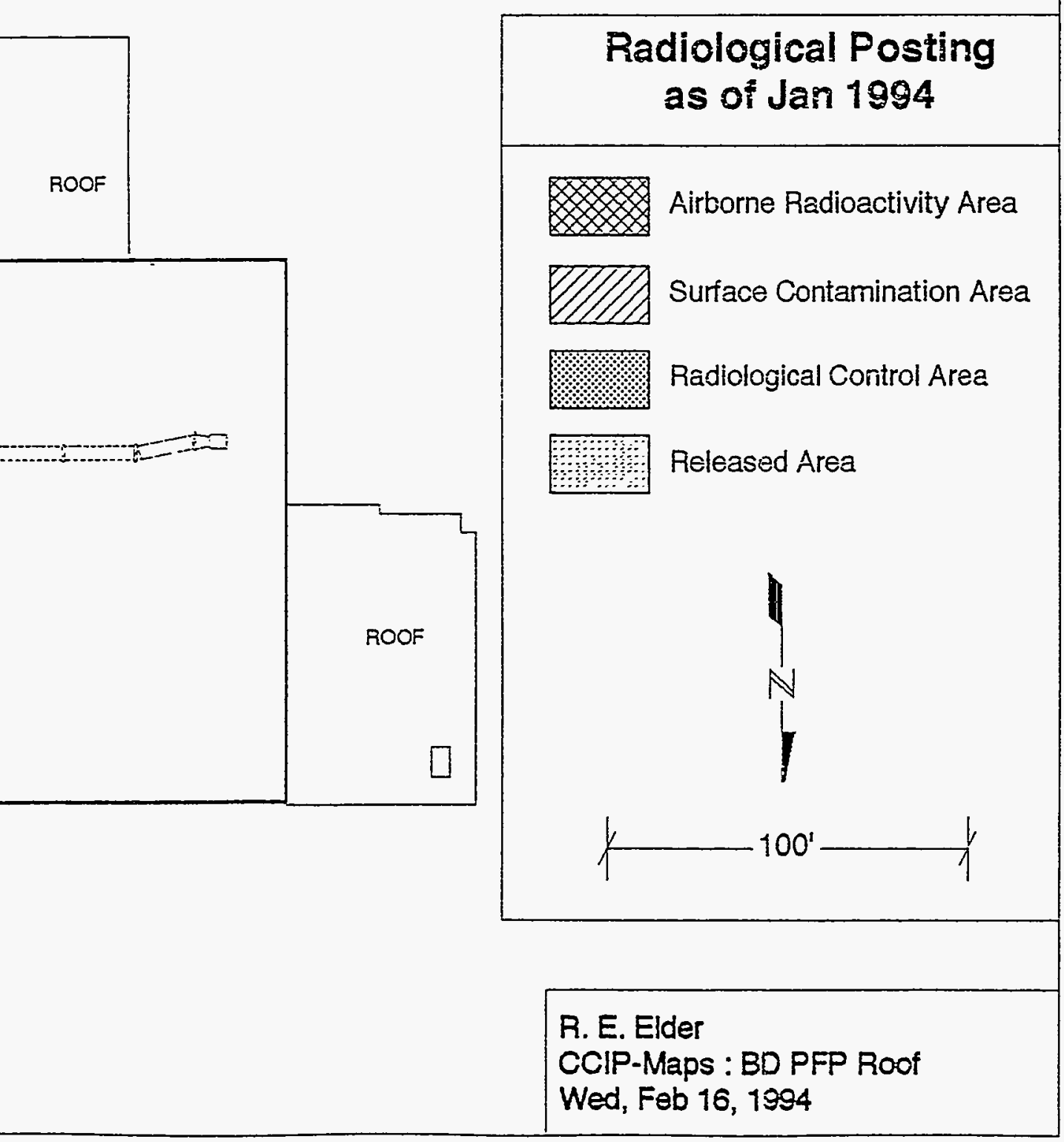


Table 4-2. Room Descriptions and Classification. (14 sheets)

\begin{tabular}{|c|c|c|c|}
\hline $\begin{array}{l}\text { Building } \\
\text { number }\end{array}$ & $\begin{array}{c}\text { Room } \\
\text { number }\end{array}$ & Description & $\begin{array}{l}\text { Contamination } \\
\text { classification }\end{array}$ \\
\hline \multirow[t]{2}{*}{$232-2$} & Bldg. & Incinerator (lay-away status) & H \\
\hline & -- & (storage, laundry, used filters) & $\cdots$ \\
\hline \multirow[t]{35}{*}{$234-52$} & Bldg. & Plutonium conversion facility & H \\
\hline & $\cdots$ & Air supple plenum chamber & H \\
\hline & 80 & Storage & C \\
\hline & 81 & Office & C \\
\hline & 82 & Office & c \\
\hline & 83 & Office & C \\
\hline & 84 & Hen's rest room & c \\
\hline & 85 & Homen's rest room & C \\
\hline & 86 & Office & C \\
\hline & 87 & office & C \\
\hline & 88 & Office & c \\
\hline & 89 & Office & c \\
\hline & 90 & Office & c \\
\hline & 92 & Pipe chase & c \\
\hline & $100-108$ & Office area & H \\
\hline & 109 & Airlock & N \\
\hline & 110 & Homen's locker room & N \\
\hline & $110-A$ & Homen's laundry hampers & N \\
\hline & $110-B$ & Homen's locker room & N \\
\hline & 111 & Men's locker room & N \\
\hline & 112 & Men's rest room & H \\
\hline & 113 & Men's rest room & N \\
\hline & 114 & -- & $\bar{N}$ \\
\hline & 115 & -- & H \\
\hline & 116 & Men's change room & H \\
\hline & 117 & Men's Laundry hampers & N \\
\hline & $117 \mathrm{~A}$ & -- & $\cdots$ \\
\hline & 120 & Homen's locker room & $N$ \\
\hline & 121 & Women's rest room & N \\
\hline & 122 & $\cdots$ & N \\
\hline & 123 & Homen's shower room & N \\
\hline & 124 & Women's change room & N \\
\hline & 126 & Personnel decontamination facility & $\mathbf{C}$ \\
\hline & 131 & Plutonium assay & $\mathrm{H}$ \\
\hline & 132 & Mass spectrometry & C \\
\hline
\end{tabular}


Table 4-2. Room Descriptions and Classification. (14 sheets)

\begin{tabular}{|c|c|c|c|}
\hline $\begin{array}{l}\text { Building } \\
\text { number }\end{array}$ & $\begin{array}{c}\text { Room } \\
\text { number }\end{array}$ & Description & $\begin{array}{l}\text { Contamination } \\
\text { classification }\end{array}$ \\
\hline \multirow{37}{*}{$\begin{array}{l}234-5 z \\
\text { (cont.) }\end{array}$} & 133 & Dark room & H \\
\hline & 134 & Mass spectrometry & H \\
\hline & 135 & Mass spectrometry & H \\
\hline & 136 & Emission spectrometry & C \\
\hline & 137 & Emission spectrometry & H \\
\hline & 139 & $\mathrm{PuO}_{2}$ sample storage & H \\
\hline & $140-A$ & Analytical support services & C \\
\hline & $140-B$ & Laboratory manager & c \\
\hline & $140-C$ & Laboratory staff chemist & C \\
\hline & 141 & Nonradioactive solution preparation & H \\
\hline & 142 & Miscellaneous storage & H \\
\hline & 143 & Haste process & H \\
\hline & 144 & Process support & H \\
\hline & 145 & Physical test & c \\
\hline & $145-A$ & Stock room & c \\
\hline & 146 & WIPP repackaging and sample analysis & H \\
\hline & 147 & Haste packaging and analysis manager & H \\
\hline & 148 & Sample analys is & H \\
\hline & 149 & Sample dissolution & H \\
\hline & 150 & Laboratory shift manager & C \\
\hline & 151 & Miscellaneous counting room & C \\
\hline & 152 & Waste concentrator & H \\
\hline & 153 & Product specification analysis & H \\
\hline & 154 & Product specification analysis & H \\
\hline & 155 & Infrared spectrophotometer & H \\
\hline & 156 & slag and crucible analysis & H \\
\hline & 157 & Product specification analys is & H \\
\hline & 158 & Instrument shop & c \\
\hline & $158-A$ & Instrument shop & C \\
\hline & 159 & Instrument shop & C \\
\hline & $159-A$ & Instrument shop & C \\
\hline & 161 & RMC shift manager & c \\
\hline & 162 & RMC shift manager & c \\
\hline & 163 & Instruments manager & C \\
\hline & 164 & RMC shift office & c \\
\hline & 165 & Calcium storage & C \\
\hline & 166 & RMC batch tanks & H \\
\hline
\end{tabular}


Table 4-2. Room Descriptions and Classification. (14 sheets)

\begin{tabular}{|c|c|c|c|}
\hline $\begin{array}{l}\text { Building } \\
\text { number }\end{array}$ & $\begin{array}{c}\text { Room } \\
\text { number }\end{array}$ & Description & $\begin{array}{l}\text { Contamination } \\
\text { classification } \\
\end{array}$ \\
\hline \multirow{37}{*}{$\begin{array}{l}234-52 \\
\text { (cont.) }\end{array}$} & 167 & RMC operations storage & c \\
\hline & 168 & RMC ready room/storage & C \\
\hline & 169 & Ash stabilization & H \\
\hline & 170 & Ash dissolution & H \\
\hline & 171 & Corridor & C \\
\hline & 172 & Maintenance shop (RMC) & C \\
\hline & 173 & Maintenance shop & c \\
\hline & 174 & SNM storage vault & H \\
\hline & 175 & SHM storage vault & C \\
\hline & 176 & Loading platform & N \\
\hline & 179 & Process support laboratory & H \\
\hline & 180 & Isotopic research & H \\
\hline & 182 & Process support (microscope) & C \\
\hline & 183 & Specialized processing & C \\
\hline & 185 & Miscellaneous storage & C \\
\hline & 186 & Storage & c \\
\hline & 187 & Open-faced hoods & H \\
\hline & 188 & Glovebox/chemical storage & H \\
\hline & 189 & Equipment storage & c \\
\hline & 190 & Radioactive material storage & H \\
\hline & 191 & Product support cold laboratory & c \\
\hline & 192 & Drum staging area & C \\
\hline & $192-A$ & SNM storage vault & $H$ \\
\hline & $192-B$ & SNM storage vault & $H$ \\
\hline & $192-\mathrm{C}$ & SHM storage vault & $H$ \\
\hline & $192-D$ & PR can storage & C \\
\hline & $192-\mathrm{S}$ & Storage & C \\
\hline & $192-N$ & NDA storage & C \\
\hline & 193 & Airlock & C \\
\hline & 194 & Storage vault & N \\
\hline & $194-A$ & Material coordinators & N \\
\hline & $194-B$ & -- & N \\
\hline & $195-M$ & -- & N \\
\hline & $195-W$ & $-\cdot$ & N \\
\hline & 196 & Waste drum storage & C \\
\hline & $196-A$ & Tool crib & H \\
\hline & 197 & Waste drum storage & C \\
\hline
\end{tabular}


Table 4-2. Room Descriptions and CTassification. (14 sheets)

\begin{tabular}{|c|c|c|c|}
\hline $\begin{array}{l}\text { Building } \\
\text { number }\end{array}$ & $\begin{array}{c}\text { Room } \\
\text { number }\end{array}$ & Description & $\begin{array}{l}\text { Contamination } \\
\text { classification }\end{array}$ \\
\hline \multirow{37}{*}{$\begin{array}{l}234-52 \\
\text { (cont.) }\end{array}$} & $197-A$ & Mixed waste drum storage & C \\
\hline & $198 \mathrm{~A}$ & Tool crib & $\mathbf{N}$ \\
\hline & 198AL & Airlock & N \\
\hline & 199 & Weld shop & H \\
\hline & 198 & Maintenance shop & H \\
\hline & 200 & Maintenance lockers & N \\
\hline & $200-A$ & Maintenance manager & N \\
\hline & 201 & Plutonium process vault & N \\
\hline & 202 & Plutonium process support lab & N \\
\hline & 205 & -- & $N$ \\
\hline & 208 & Hork authorization system & N \\
\hline & 208-A & Planner/scheduler & N \\
\hline & 208-B & Planner/scheduler & H \\
\hline & 209 & Planner/scheduler & N \\
\hline & $209-A$ & Planner/scheduler & N \\
\hline & $209-B$ & Planner/scheduler & N \\
\hline & 210 & Men's change room hampers & N \\
\hline & 211 & Men's change room & N \\
\hline & 212 & Men's rest room & N \\
\hline & $212 A$ & $\cdots$ & N \\
\hline & 213 & Airlocks & $\mathrm{N}$ \\
\hline & 214 & Men's showers & H \\
\hline & 215 & Men's rest room & H \\
\hline & 216 & Men's change room & N \\
\hline & 217 & Analytical lab manager & N \\
\hline & $217-A$ & Maintenance office & N \\
\hline & $218-A$ & Maintenance office & N \\
\hline & $218-B$ & Maintenance office & H \\
\hline & $218-C$ & Maintenance office & N \\
\hline & 218-D & Maintenance office & N \\
\hline & 218-E & Maintenance office & H \\
\hline & $218-F$ & Maintenance office & N \\
\hline & $221-A$ & Heal th Physics office & C \\
\hline & $221-8$ & Sealer repair shop & C \\
\hline & $221-C$ & Radiochemical standards laboratory & $H$ \\
\hline & $221-D$ & Rediochemical standards laboratory & H \\
\hline & $221-E$ & Radiochemical standards laboratory & H \\
\hline
\end{tabular}


Tab7e 4-2. Room Descriptions and Classification. (14 sheets)

\begin{tabular}{|c|c|c|c|}
\hline $\begin{array}{l}\text { Building } \\
\text { number }\end{array}$ & $\begin{array}{c}\text { Room } \\
\text { number }\end{array}$ & Description & $\begin{array}{l}\text { Contamination } \\
\text { classification }\end{array}$ \\
\hline \multirow{37}{*}{$\begin{array}{l}234-52 \\
\text { (cont.) }\end{array}$} & $221-F$ & Health Physics office & K \\
\hline & $221-G$ & Health Physics office & N \\
\hline & 224 & Corridor & H \\
\hline & 225 & Product handling vault & $H$ \\
\hline & 227 & Product load-in/load-out & H \\
\hline & 228-A & RKC production line & H \\
\hline & 228-A & RHC production line & -- \\
\hline & $228-8$ & RMC production line & H \\
\hline & $228-\mathrm{C}$ & RMC production line & H \\
\hline & 229 & RMC control room & N \\
\hline & $230-A$ & RMC seal-out glovebox & H \\
\hline & $230-B$ & RMC recoverable powder glovebox & H \\
\hline & $230-c$ & Glovebox HC-60 hydrolys is & H \\
\hline & 231 & -- & C \\
\hline & 232 & HF scrubber cell & H \\
\hline & $232 A$ & HF scrubber & H \\
\hline & 233 & RMA control room & c \\
\hline & $233 A$ & Electrical Room & C \\
\hline & 234 & Trash compactor & c \\
\hline & $234-A$ & NDA measurement of drums & c \\
\hline & 235 & Airlock & C \\
\hline & 235-A1 & RMA oxide line (inactive) & H \\
\hline & $235-A 2$ & RMA oxide line (inactive) & h \\
\hline & $235-A 3$ & RMA oxide line (inactive) & H \\
\hline & $235-8$ & Recoverable material stabilization & H \\
\hline & $235-c$ & Waste repackaging & H \\
\hline & $235-0$ & Waste drum storage/RADTU & H \\
\hline & $235-E$ & RADTU filter room & H \\
\hline & 236 & PR can storage vault & H \\
\hline & $236 \mathrm{~A}$ & $\cdots$ & C \\
\hline & $236 B$ & -- & C \\
\hline & $236 \mathrm{C}$ & $\because$ & C \\
\hline & 237 & $\because$ & C \\
\hline & 238 & $\cdots$ & C \\
\hline & 239 & -- & C \\
\hline & 240 & $\cdots$ & $c$ \\
\hline & 241 & $\cdots$ & C \\
\hline
\end{tabular}


Table 4-2. Room Descriptions and Classification. (14 sheets)

\begin{tabular}{|c|c|c|c|}
\hline $\begin{array}{l}\text { Building } \\
\text { number }\end{array}$ & $\begin{array}{c}\text { Room } \\
\text { number }\end{array}$ & Description & $\begin{array}{l}\text { Contamination } \\
\text { classification }\end{array}$ \\
\hline \multirow{37}{*}{$\begin{array}{l}234-52 \\
\text { (cont.) }\end{array}$} & 242 & Ready room shipping/receiving & C \\
\hline & $242 A$ & Shift manager prod. handling & C \\
\hline & 2428 & Shift supervisor prod. handling & C \\
\hline & 245 & Corridor & C \\
\hline & 247 & $\cdots$ & C \\
\hline & 248 & -- & C \\
\hline & 249 & -- & C \\
\hline & Mezz & Mezzaninie columns $12-22$ & C \\
\hline & 250 & Office/storage & N \\
\hline & $251 A$ & office/storage & N \\
\hline & $251 \mathrm{~B}$ & office/storage & N \\
\hline & 252 & office/storage & N \\
\hline & 253 & office/storage & N \\
\hline & 254 & Office/storage & N \\
\hline & 255 & Janitors room & $\mathbf{N}$ \\
\hline & 260 & Duct level & N \\
\hline & $260 \mathrm{~A}$ & Airlock & N \\
\hline & 261 & Storage & N \\
\hline & 262 & Duct level & H \\
\hline & 263 & Duct level & H \\
\hline & 264 & Duct level & H \\
\hline & 265 & Duct level & N \\
\hline & 266 & Electrical room & H \\
\hline & 267 & Telephone switchgear room & N \\
\hline & 268 & Storage room & N \\
\hline & 269 & Battery storage & $\mathbf{N}$ \\
\hline & 270 & Duct level & C \\
\hline & $270 A$ & Airlock & N \\
\hline & 271 & -- & C \\
\hline & 272 & Duct level & C \\
\hline & 300 & Foyer & H \\
\hline & 301 & Maintenance engineers & H \\
\hline & $301-A$ & Process engineers & N \\
\hline & $301-B$ & Staff assistant & N \\
\hline & $301-C$ & Process engineer & H \\
\hline & $301-D$ & $\cdots$ & N \\
\hline & 302 & Chemical storage & H \\
\hline
\end{tabular}


Table 4-2. Room Descriptions and Classification. (14 sheets)

\begin{tabular}{|c|c|c|c|}
\hline $\begin{array}{l}\text { Building } \\
\text { number }\end{array}$ & $\begin{array}{c}\text { Room } \\
\text { number }\end{array}$ & Description & $\begin{array}{l}\text { Contamination } \\
\text { classification }\end{array}$ \\
\hline \multirow{37}{*}{$\begin{array}{l}234-5 Z \\
\text { (cont.) }\end{array}$} & 303 & Process engineer & H \\
\hline & 303-A & Process engineer & N \\
\hline & 303-B & Process engineer & N \\
\hline & $303-c$ & Process engineer & H \\
\hline & 303-D & Repository & N \\
\hline & $303-E$ & Process engineer & $\mathbf{N}$ \\
\hline & $303-F$ & Envir. and effluents engineering manager & N \\
\hline & $303-6$ & Process engineers & H \\
\hline & $303-H$ & Process engineers & H \\
\hline & $303-J$ & Process engineers & N \\
\hline & $303-K$ & Secretary (engineering) & N \\
\hline & $303-L$ & Process support manager & H \\
\hline & $303-M$ & Process control manager & N \\
\hline & 304 & Plastic shop & N \\
\hline & $304-A$ & Store room office & N \\
\hline & $304-8$ & Property specialist & N \\
\hline & 305 & Lunch room & H \\
\hline & $305 A$ & -- & $N$ \\
\hline & 306 & Conference room & N \\
\hline & 306-A & Computer room & N \\
\hline & 307 & Airlock & $N$ \\
\hline & 308 & Duct level & H \\
\hline & 309 & E-4 filter room & $H$ \\
\hline & 310 & E-4 filter room & H \\
\hline & 311 & E-3 filter room & H \\
\hline & 312 & E-3 filter room & H \\
\hline & 313 & E-3 filter room & H \\
\hline & 314 & E-3 filter room & H \\
\hline & 315 & E-3 filter room & H \\
\hline & 316 & E-3 filter room & H \\
\hline & 318 & E-3 filter room & H \\
\hline & 319 & Supply fans & C \\
\hline & 320 & Filterboxes & H \\
\hline & 321 & Steam supply & N \\
\hline & $321-A$ & Power control room & C \\
\hline & $321-B$ & Power mansger & N \\
\hline & $321-c$ & Change room & N \\
\hline
\end{tabular}


Table 4-2. Room Descriptions and Classification. (14 sheets)

\begin{tabular}{|c|c|c|c|}
\hline $\begin{array}{l}\text { Building } \\
\text { number }\end{array}$ & $\begin{array}{l}\text { Room } \\
\text { number }\end{array}$ & Description & $\begin{array}{l}\text { Contamination } \\
\text { classification }\end{array}$ \\
\hline \multirow{36}{*}{$\begin{array}{l}234-52 \\
\text { (cont.) }\end{array}$} & 322 & Electrical shop & N \\
\hline & 323 & Electrical manager & N \\
\hline & 324 & Janitor closet & H \\
\hline & 325 & Process engineer & N \\
\hline & 326 & Airlock passageway & N \\
\hline & 327 & Hamper room & N \\
\hline & 328 & Men's change room & N \\
\hline & 329 & Men's toilet & N \\
\hline & 330 & Men's shower & N \\
\hline & 331 & Men's locker room & N \\
\hline & 332 & Men's locker room entry & H \\
\hline & 333 & Men's toilet & N \\
\hline & 333-A & Homen's toilet & H \\
\hline & 334 & Calcium storage & N \\
\hline & 335 & Calcium storage & N \\
\hline & 336 & Chemical makeup & H \\
\hline & 337 & Chemical makeup & N \\
\hline & 338 & Het chemical storage & N \\
\hline & 339 & Men's Shower & N \\
\hline & 340 & Instrument shop & N \\
\hline & 341 & IRH/SSDH manager & N \\
\hline & 342 & Maintenance engineer & N \\
\hline & 343 & Haintenance engineer & N \\
\hline & 344 & Hal lway & N \\
\hline & $344-A$ & Radiation engineer & N \\
\hline & 345 & Activity engineer & N \\
\hline & 346 & Administrative specialist & N \\
\hline & 347 & IRM/SSDM engineer & N \\
\hline & 348 & IRM/SSDM engineer & N \\
\hline & 349 & -- & N \\
\hline & 350 & -- & N \\
\hline & 351 & Entrance to computer room & H \\
\hline & 390 & Airlock & N \\
\hline & 682 & Airlock & C \\
\hline & 878AL & Airlock & N \\
\hline & 879AL & Ajrlock & N \\
\hline
\end{tabular}


Table 4-2. Room Descriptions and Classification. (14 sheets)

\begin{tabular}{|c|c|c|c|}
\hline $\begin{array}{l}\text { Building } \\
\text { number }\end{array}$ & $\begin{array}{c}\text { Room } \\
\text { number }\end{array}$ & Description & $\begin{array}{l}\text { Contamination } \\
\text { classification }\end{array}$ \\
\hline \multirow[t]{29}{*}{$234-5 Z A$} & 701 & Men's change room & H \\
\hline & 702 & -- & N \\
\hline & 703 & $\cdots$ & H \\
\hline & 704 & -- & N \\
\hline & 705 & -- & N \\
\hline & 706 & -- & N \\
\hline & 707 & -- & $\mathbf{N}$ \\
\hline & 708 & Storage & H \\
\hline & 709 & Corridor & K \\
\hline & 710 & Storage & N \\
\hline & 711 & Storage & N \\
\hline & 712 & -- & H \\
\hline & 713 & Fire sprinkler riser room & $H$ \\
\hline & 714 & Computer room & N \\
\hline & 715 & Health Physics technician WRAM station & N \\
\hline & 716 & RUP posting area & N \\
\hline & 717 & Self survey station & N \\
\hline & 718 & Locker room/lobby & N \\
\hline & 719 & Lobby & H \\
\hline & 720 & RCA entrance/exit thoroughfare & N \\
\hline & 722 & RCA exit security portal & H \\
\hline & 724 & RCA exit security & H \\
\hline & 726 & RCA entry portal & N \\
\hline & 728 & Security check station & N \\
\hline & 730 & Corridor & $\mathbf{N}$ \\
\hline & 731 & Corridor & N \\
\hline & 732 & - & N \\
\hline & 733 & Corridor & N \\
\hline & 734 & -- & N \\
\hline \multirow[t]{8}{*}{$236-2$} & 10 & Corridor & $H$ \\
\hline & 11 & Corridor & $H$ \\
\hline & 12 & Process cell & H \\
\hline & $12 A$ & Mezzanine & H \\
\hline & 13 & Corridor & H \\
\hline & 14 & Corridor & H \\
\hline & 15 & Helding shop & H \\
\hline & 16 & Maintenance shop (PRF) & H \\
\hline
\end{tabular}


Table 4-2. Room Descriptions and Classification. (14 sheets)

\begin{tabular}{|c|c|c|c|}
\hline $\begin{array}{l}\text { Building } \\
\text { number }\end{array}$ & $\begin{array}{c}\text { Room } \\
\text { number }\end{array}$ & Description & $\begin{array}{l}\text { Contamination } \\
\text { classification }\end{array}$ \\
\hline \multirow{29}{*}{$\begin{array}{l}236-2 \\
\text { (cont.) }\end{array}$} & 17 & Maintenance manager's office & H \\
\hline & 18 & Elevator access/storage & H \\
\hline & 19 & NDA counting room & H \\
\hline & 20 & Corridor/filterboxes & H \\
\hline & 21 & Hest corridor & H \\
\hline & 25 & East corridor & H \\
\hline & 26 & Filter room & H \\
\hline & 27 & Maintenance glovebox & H \\
\hline & 28 & Glovebox room & $H$ \\
\hline & 30 & Corridor & H \\
\hline & 31 & West corridor & H \\
\hline & 33 & East corridor & H \\
\hline & 34 & Instrument shop & H \\
\hline & 35 & Electrical room & H \\
\hline & 36 & Compressor room & H \\
\hline & 37 & Storage room & H \\
\hline & 38 & Rest room & C \\
\hline & 40 & Chemical preparation & H \\
\hline & 41 & Miscellaneous treatment & $\mathrm{H}$ \\
\hline & 42 & Column room & $\mathrm{H}$ \\
\hline & 43 & slag and crucible gloveboxes & H \\
\hline & $43 A$ & $-\cdot$ & c \\
\hline & 44 & Column room & C \\
\hline & 45 & Shift manager's office & C \\
\hline & 46 & Shift engineer's office & C \\
\hline & 47 & Corridor & C \\
\hline & 50 & Column glovebox & h \\
\hline & 60 & Colum glovebox & H \\
\hline & 73 & Mechanical Roon & C \\
\hline $234-2 B$ & Bldg. & Miscellaneous storage for construction forces & N \\
\hline $234-2 C$ & Bldg. & Used for loading dock for waste drums and SNM containers & c \\
\hline $241-z$ & Bldg. & Liquid waste collection tanks in an underground concrete vault & H \\
\hline $241-Z-R B$ & Bldg. & Waste Hater retention bas in (no longer used) & N \\
\hline $241-2 A$ & Bldg. & 241-2 Tank sampl ing & H \\
\hline $241-2 B$ & Bldg. & Curbed concrete pad housing the $D-9$ caustic tank & $N$ \\
\hline $241-26$ & $\cdots$ & Change Room & C \\
\hline $241-2-361$ & Bldg. & Underground liquid waste settling tank (no longer used) & N \\
\hline
\end{tabular}


Table 4-2. Room Descriptions and Classification. (14 sheets)

\begin{tabular}{|c|c|c|c|}
\hline $\begin{array}{l}\text { Building } \\
\text { number }\end{array}$ & $\begin{array}{l}\text { Room } \\
\text { number }\end{array}$ & Description & $\begin{array}{l}\text { Contamination } \\
\text { classification }\end{array}$ \\
\hline \multirow[t]{2}{*}{$242-2$} & Bldg. & WT Facility (lay-away status) & H \\
\hline & 108 & Airlock & H \\
\hline $243-2 A$ & -- & Tank and sump pit & C \\
\hline \multirow[t]{5}{*}{$243-2$} & 401 & $\cdots$ & C \\
\hline & 402 & -- & C \\
\hline & 403 & -- & C \\
\hline & 404 & Drum washer & c \\
\hline & 405 & Drum storage & C \\
\hline $243-28$ & $\cdots$ & Cooling equipment pad & C \\
\hline $252-z-1$ & Bldg. & Electrical transformers & N \\
\hline $252-z-2$ & Bldg. & Electrical transformers & C \\
\hline $267-2$ & Bldg. & Valve house for water supply to a $234-52$ sprinkler system & N \\
\hline $270-z$ & Bldg. & PRF support facility (office building) & N \\
\hline \multirow[t]{13}{*}{$291-z$} & Bldg. & Ventilation exhaust fan house & C \\
\hline & -- & Cable vault & C \\
\hline & 500 & Electrical room & c \\
\hline & 501 & Compressor room & C \\
\hline & 502 & Exhaust plenum & C \\
\hline & 503 & $\cdots$ & $N$ \\
\hline & 504 & $\because$ & C \\
\hline & 505 & $\cdots$ & C \\
\hline & 506 & -- & C \\
\hline & 507 & -- & C \\
\hline & 508 & - & C \\
\hline & 509 & Fan room & C \\
\hline & 510 & $\cdots$ & N \\
\hline $291-2-1$ & Bldg. & Ventilation exhaust stack for $232-2,234-52$, and $242-2$ Buildings & c \\
\hline $296-z-3$ & Bldg. & Ventilation exhaust stack for $241-2$ vault and tanks & H \\
\hline $296-2-5$ & Bldg. & Ventilation exhaust stack for $2736-2$ Building & N \\
\hline $296-2-6$ & Bldg. & Ventilation exhaust stack for $2736-2$ Building & H \\
\hline $2503-2$ & Bldg. & Electrical distribution system for PFP (switch yard) & H \\
\hline $2701-z$ & Bldg. & Hooden building (no longer used) & $N$ \\
\hline $2701-Z A$ & Bldg. & Central station alarm facility & H \\
\hline $2701-2 B$ & Bldg. & Security badge house & H \\
\hline $2701-20$ & Bldg. & Security check point station & $\cdots$ \\
\hline $2702-2$ & Bldg. & Microwave tower & H \\
\hline $2704-2$ & Bldg. & PFP Support facility (office building) & $\mathrm{H}$ \\
\hline $2705-2$ & Bldg. & Badge house & $\cdots$ \\
\hline $2712-2$ & Bldg. & 291-2-1 stack sampler power generators (diesel driven) & c \\
\hline $2715-2$ & Bldg. & Paint and solvent storage & N \\
\hline $2715-Z L$ & Bldg. & Drum storage & N \\
\hline
\end{tabular}


Table 4-2. Room Descriptions and Classification. (14 sheets)

\begin{tabular}{|c|c|c|c|}
\hline $\begin{array}{l}\text { Building } \\
\text { number }\end{array}$ & $\begin{array}{c}\text { Room } \\
\text { number }\end{array}$ & Description & $\begin{array}{l}\text { Contamination } \\
\text { classification }\end{array}$ \\
\hline $2721-2$ & Bldg. & Emergency electric power generators (diesel driven) & H \\
\hline $2722-2$ & Bldg. & Concrete pad for truck load-out station & H \\
\hline $2725-2$ & Bldg. & Laundry storage & H \\
\hline $2727-2$ & Bldg. & Storage of miscellaneous operations and laboratory equipment & H \\
\hline $2729-2$ & Bldg. & Storage of miscellaneous maintenance materials & N \\
\hline $2731-2$ & Bldg. & Storage of empty flushed plutonium drums & H \\
\hline $2731-2 A$ & Bldg. & Laundry storage & C \\
\hline $2734-2$ & Bldg. & Gas bottle storage & H \\
\hline $2734-2 A$ & Bldg. & Liquid argon storage & H \\
\hline $2734-28$ & Bldg. & Flammable gas storage (out of service) & N \\
\hline $2734-2 C$ & Bldg. & Gas bottle storage (out of service) & H \\
\hline $2734-20$ & Bldg. & Argon and oxygen bottle supply (out of service) & H \\
\hline $2734-2 F$ & Bldg. & Standby gas bottle supply (out of service) & H \\
\hline $2734-2 G$ & Bldg. & Standby gas bottle supply (out of service) & $H$ \\
\hline $2734-2 H$ & Bldg. & Argon bottle supply (out of service) & $\mathbf{H}$ \\
\hline $2734-\mathrm{JJ}$ & Bldg. & Liquid nitrogen storage tank & N \\
\hline $2734-2 K$ & Bldg. & Gas bottle storage, acetylene, argon, oxygen & $H$ \\
\hline $2734-2 L$ & Bldg. & HF ges bottles and supply piping & C \\
\hline $2735-2$ & Bldg. & Chemical storage of $\mathrm{HNO}^{3}$, and ANN & H \\
\hline \multirow[t]{5}{*}{$2736-2$} & 8ldg. & SNM storage & H \\
\hline & 1 & Storage of product/recoverable SNM & H \\
\hline & 2 & Lard can/cubicle SNM storage & H \\
\hline & 3 & Storage of product/recoverable SNM & H \\
\hline & 4 & Storage of product/recoverable SNM & H \\
\hline $2736-2 A$ & Bldg. & $2736-2$ exhaust fans and $65 \mathrm{~kW}$ diesel generator & H \\
\hline \multirow[t]{14}{*}{$2736-2 B$} & Bldg. & Shipping and receiving SNM & H \\
\hline & 600 & Mech room & H \\
\hline & 602 & Mech room & H \\
\hline & 603 & -- & N \\
\hline & 604 & Security computer room & N \\
\hline & 605 & Office & N \\
\hline & 606 & Office & N \\
\hline & 607 & Office & H \\
\hline & 608 & Vestibule & N \\
\hline & 610 & Receptionist office & N \\
\hline & 691 & Office & N \\
\hline & 612 & Office & N \\
\hline & 613 & -- & H \\
\hline & 614 & Homen's change room & N \\
\hline
\end{tabular}


Table 4-2. Room Descriptions and Classification. (14 sheets)

\begin{tabular}{|c|c|c|c|}
\hline $\begin{array}{l}\text { Building } \\
\text { number }\end{array}$ & $\begin{array}{l}\text { Room } \\
\text { number }\end{array}$ & Description & $\begin{array}{c}\text { Contamination } \\
\text { classification } \\
\end{array}$ \\
\hline \multirow{33}{*}{$\begin{array}{l}\text { 2736-2B } \\
\text { (cont.) }\end{array}$} & 615 & Homen's change room & N \\
\hline & 616 & Homen's change room & N \\
\hline & 618 & Men's change room & $N$ \\
\hline & 619 & Men's change room & H \\
\hline & 620 & Lunchroom & H \\
\hline & 621 & Men's change room & N \\
\hline & 622 & Men's change room & H \\
\hline & 623 & UPS room & N \\
\hline & 624 & Corridor & H \\
\hline & 625 & Corridor & C \\
\hline & 626 & Janitor closet & C \\
\hline & 627 & Homen's rest room & C \\
\hline & 628 & Men's rest room & C \\
\hline & 629 & Mardix & C \\
\hline & 630 & Decontamination room & C \\
\hline & 631 & Health Physics office & C \\
\hline & 632 & Applied technology chemist's office & C \\
\hline & 633 & Corridor & C \\
\hline & 634 & Shift manager & C \\
\hline & 635 & Operator ready room & c \\
\hline & 636 & Repackaging room & H \\
\hline & 637 & NDA Laboratory & $\mathrm{H}$ \\
\hline & 638 & Packaging room & H \\
\hline & 638A & -- & H \\
\hline & 639 & Safety shower room & H \\
\hline & 640 & Supply storage & H \\
\hline & 641 & Receiving room & H \\
\hline & $641 \mathrm{~A}$ & $\cdots$ & H \\
\hline & 642 & Shipping room & H \\
\hline & 643 & Personnel entry & C \\
\hline & 644 & Material passageway & C \\
\hline & 645 & -- & H \\
\hline & 646 & $\cdots$ & N \\
\hline
\end{tabular}


Table 4-2. Room Descriptions and Classification. (14 sheets)

\begin{tabular}{|c|c|l|c|}
\hline $\begin{array}{c}\text { Building } \\
\text { number }\end{array}$ & $\begin{array}{c}\text { Room } \\
\text { number }\end{array}$ & \multicolumn{1}{|c|}{ Description } & $\begin{array}{c}\text { Contamination } \\
\text { classification }\end{array}$ \\
\hline $2736-2 C$ & Bldg. & Loading dock & $\mathrm{N}$ \\
\hline $2902-\mathrm{Z}$ & Bldg. & Elevated sanitary water tank and valve pit & $\mathrm{N}$ \\
\hline $2904-\mathrm{ZA}$ & Bldg. & $\begin{array}{l}\text { Sheet metal structure housing 216-Z-20 crib stream effluent } \\
\text { sampl ing, flow measurement, pH and alpha monitoring equipment }\end{array}$ & $\mathrm{N}$ \\
\hline $2904-2 \mathrm{~B}$ & Bldg. & $\begin{array}{l}\text { Sheet metel structure housing 216-Z-20 crib stream effluent } \\
\text { sampl ing, flow measurement, pH and alpha monitoring equipment }\end{array}$ & $\mathrm{N}$ \\
\hline
\end{tabular}

ANN = Aluminum nitrate nonhydrate.

$C=$ "Cold" process support area.

$H=$ "Hot" process area.

$H F=$ Hydrofluoric acid.

IRM = Information Resource Management (Department).

$N=$ Not contaminated.

NDA = Nondestructive assay.

$P R=$ Product removal.

PFP $=$ Plutonium Finishing Plant.

PRF = Plutonium Reclamation Facility.

RADTU = Radioactive Acid Digestion Test Unit.

RCA = Radiological controlled area.

RMA = Renote mechanical "A" line.

RMC = Remote mechanical "C" line.

RHP $=$ Rediological work permit.

SNM = Special nuclear material.

SSDM = structured system development methodology (data management).

SWP $=$ Special work permit (protective clothing).

UPS = Uninterruptible power supply.

HIPP $=$ Waste Isolation Pilot Plant.

WRAM = West inghouse Hanford Company radiation area management. 


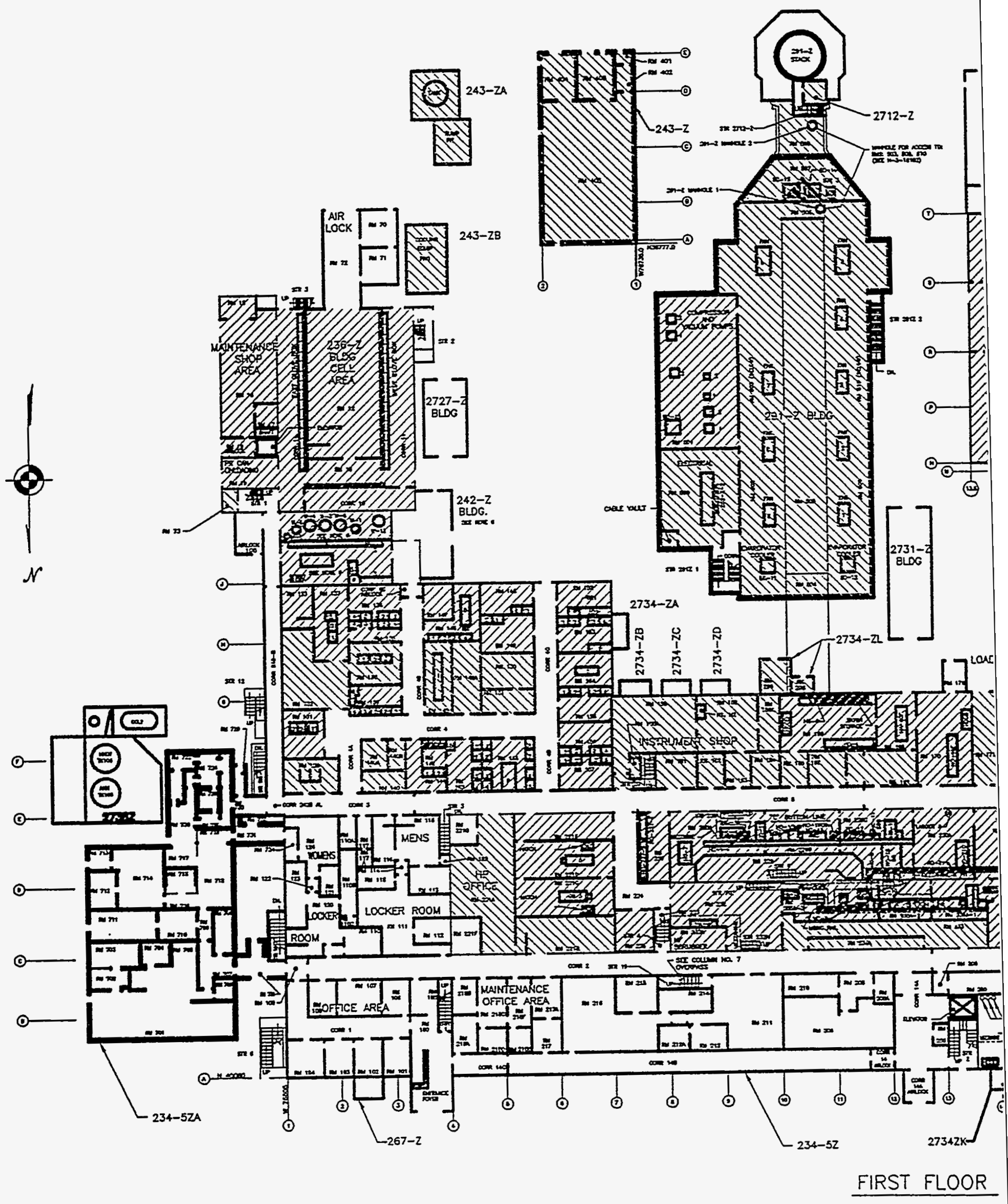




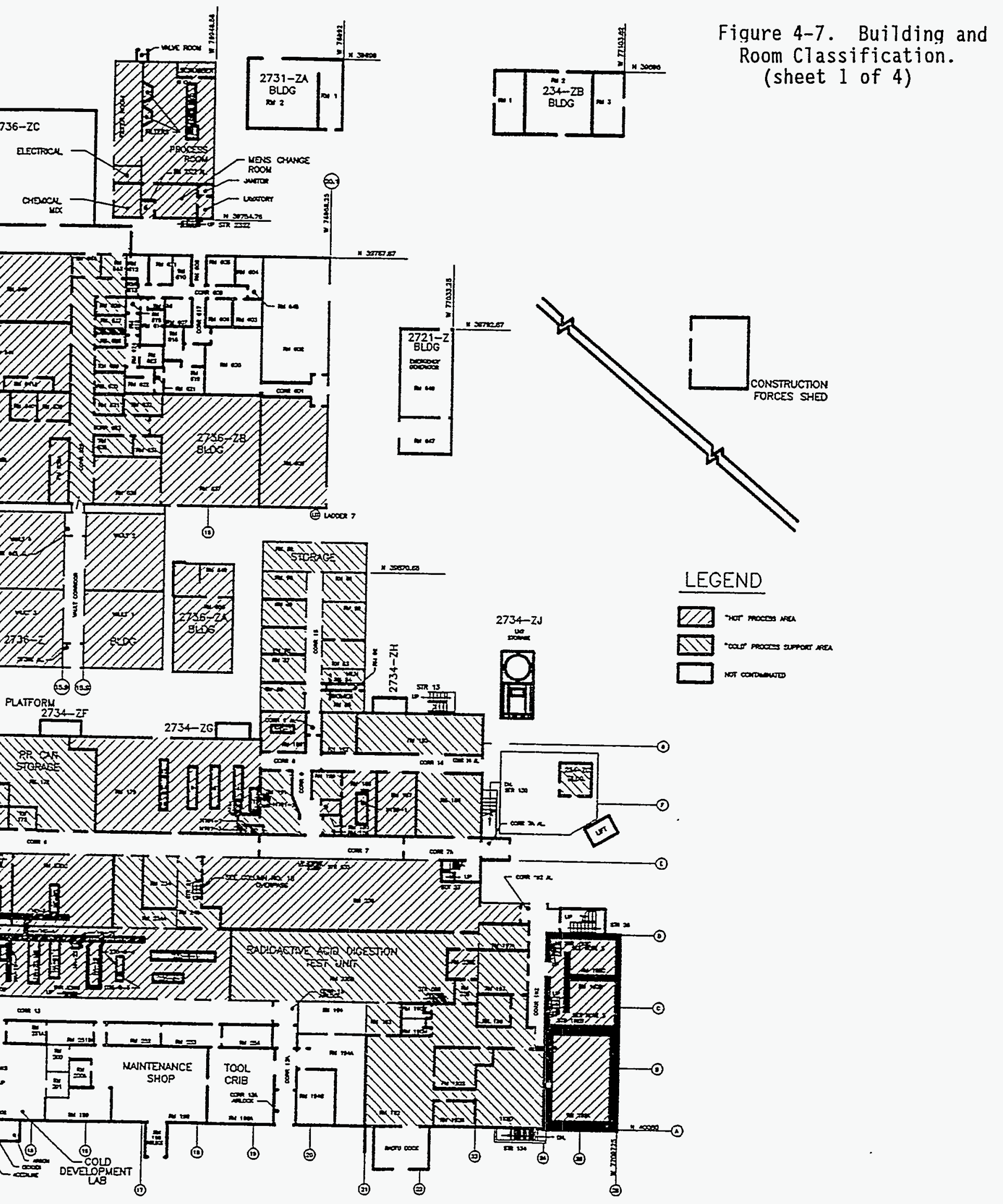



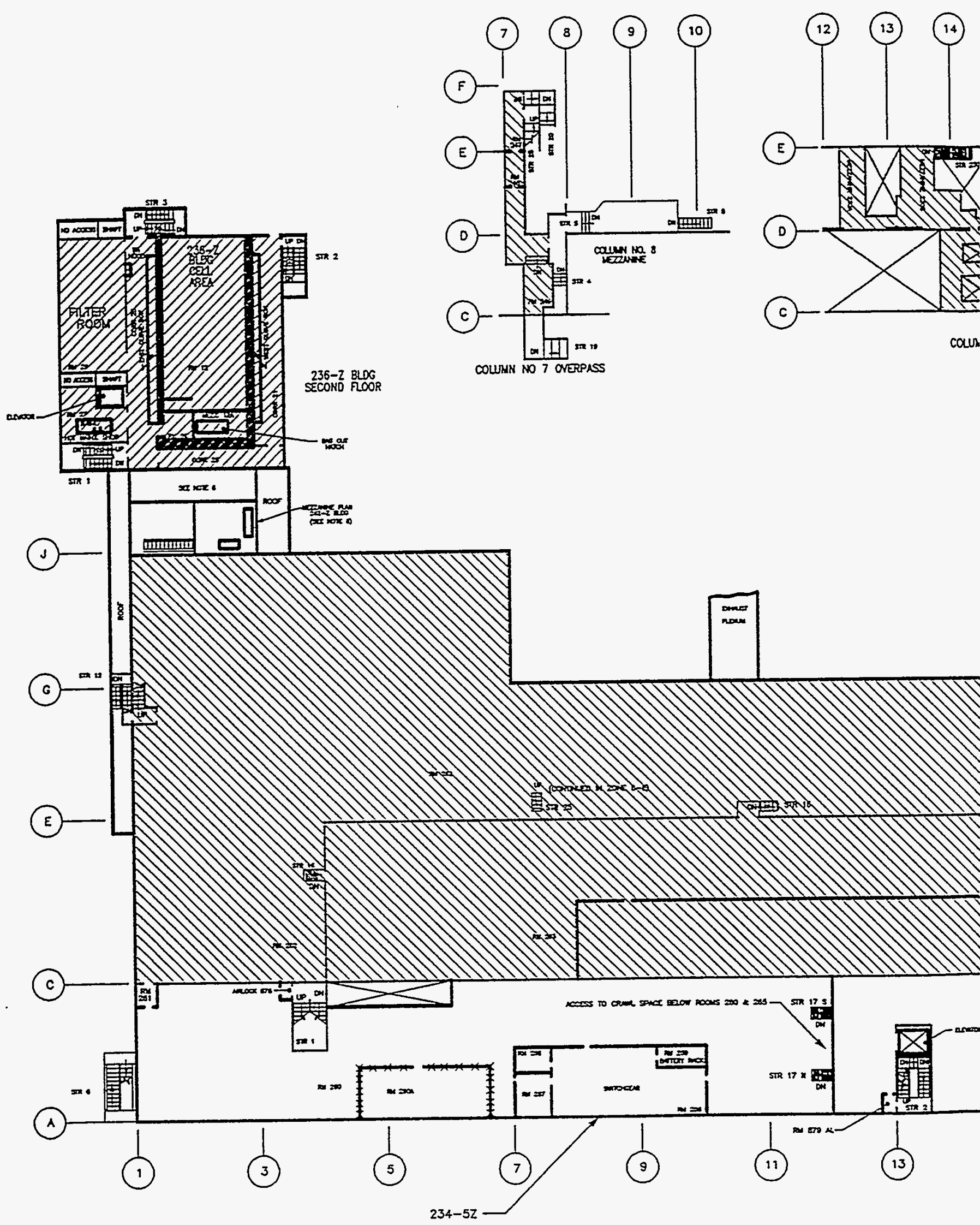

DUCT LEVEL PLAN 


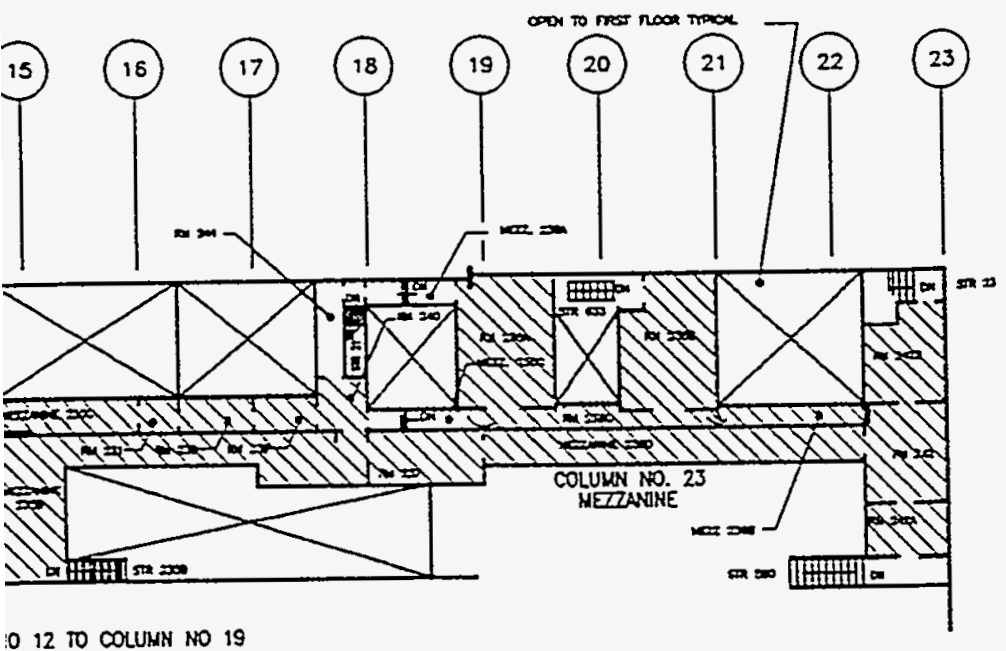

012 TO COLUMN NO 19
WHC-EP-0829

Figure 4-7. Building and Room Classification. (sheet 2 of 4 )

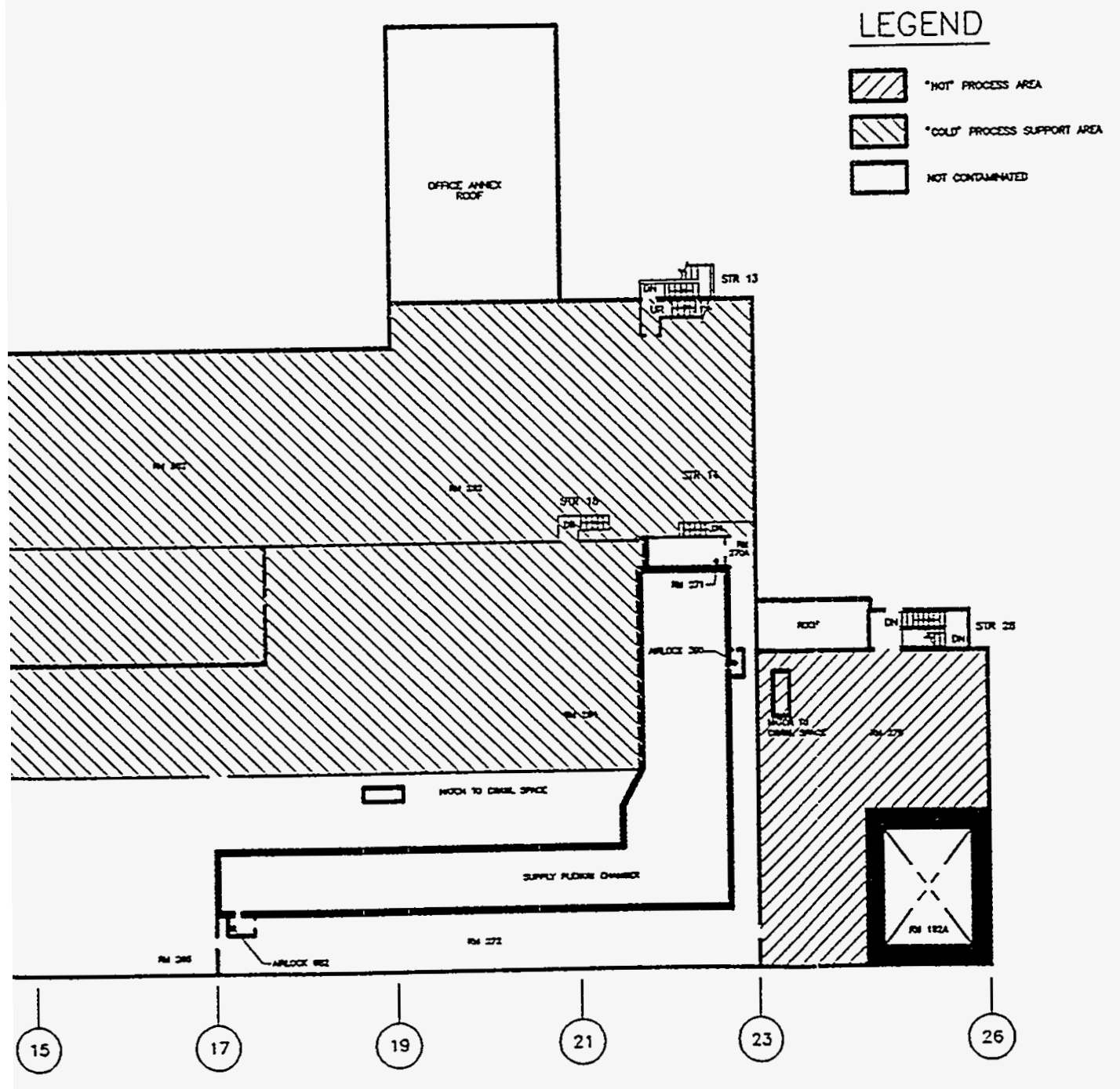




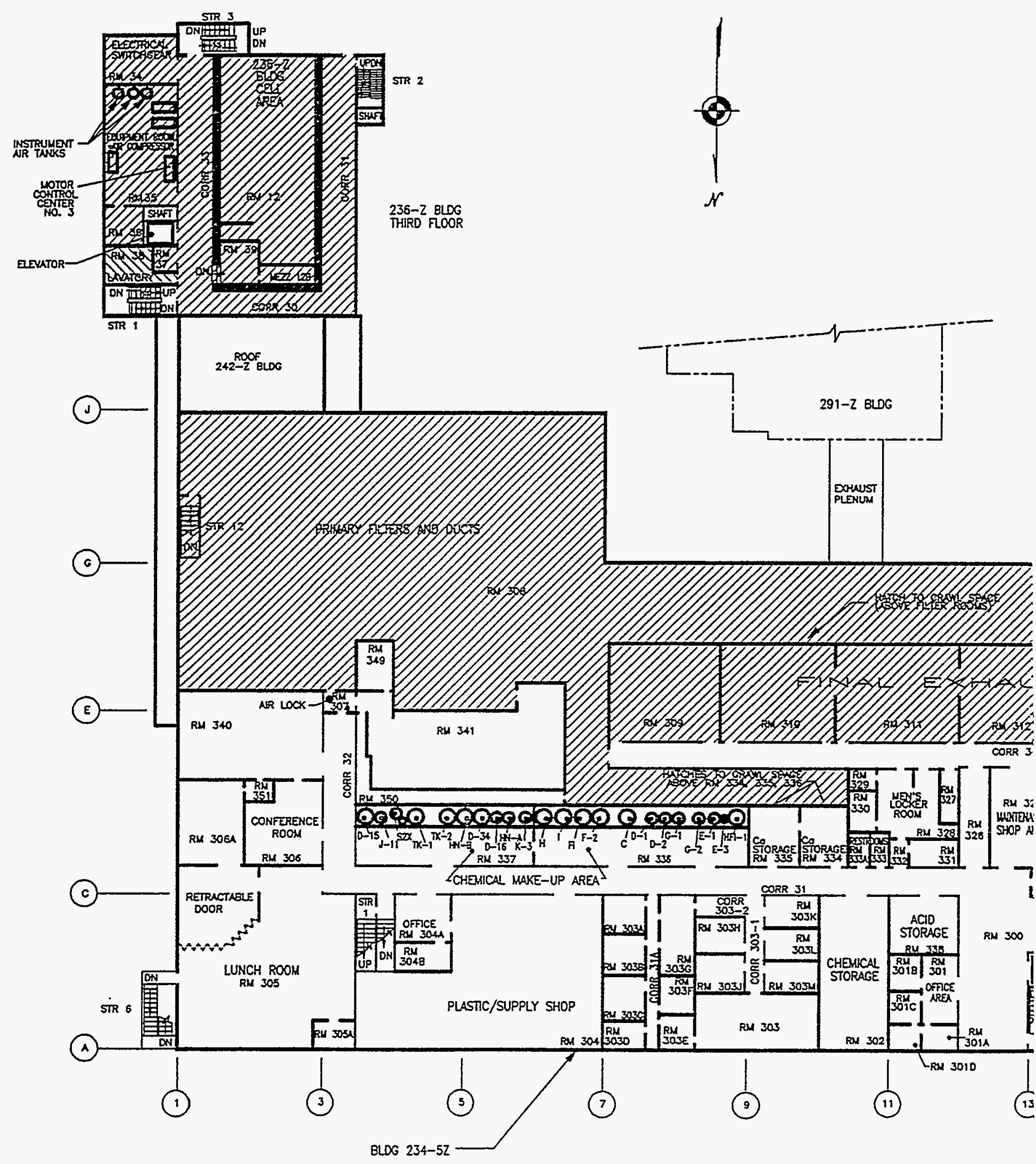


232-Z BLDG

SECOND FLOOR

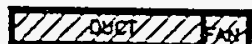

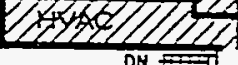

$\operatorname{STR}^{\mathrm{DN}} 32 \mathrm{C}$
WHC-EP-0829

Figure 4-7. Building and Room Classification. (sheet 3 of 4 )

LEGEND

EIA

III

$\square$
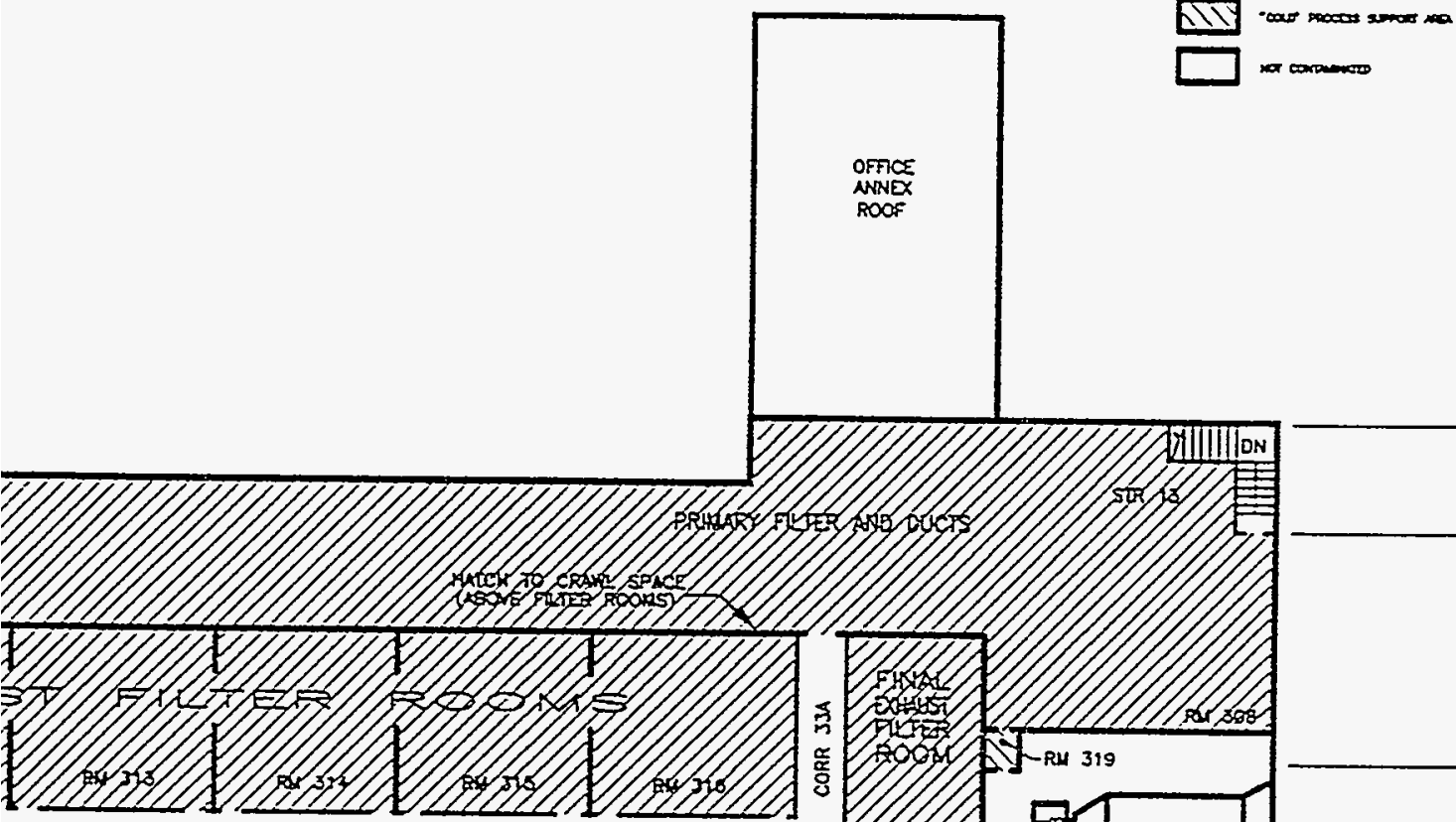

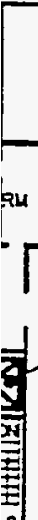

MANTENANCE

SHOP AREA

Q4 323

322

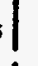

$\sqrt{221-8}$

RM 321
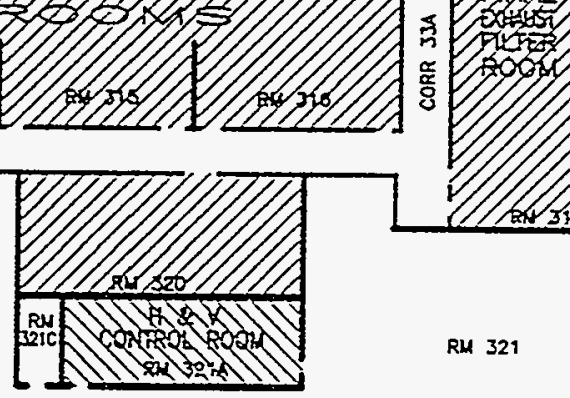

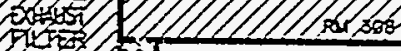

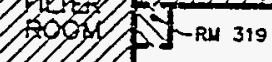
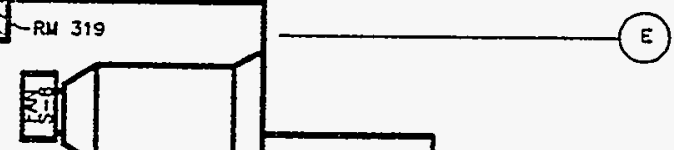

E

\section{1}
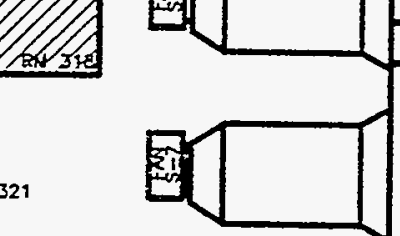
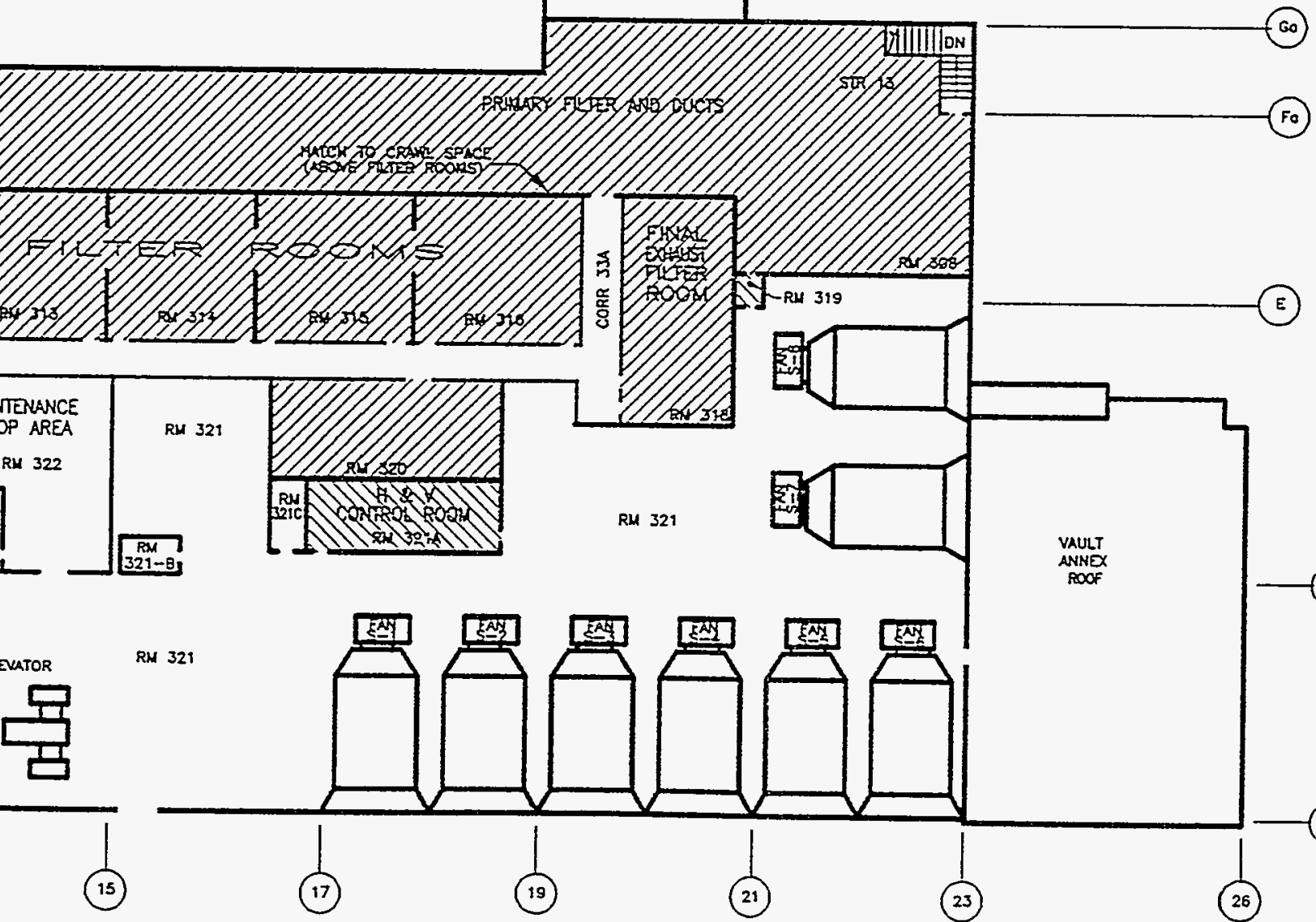

Fo.

R PLAN 


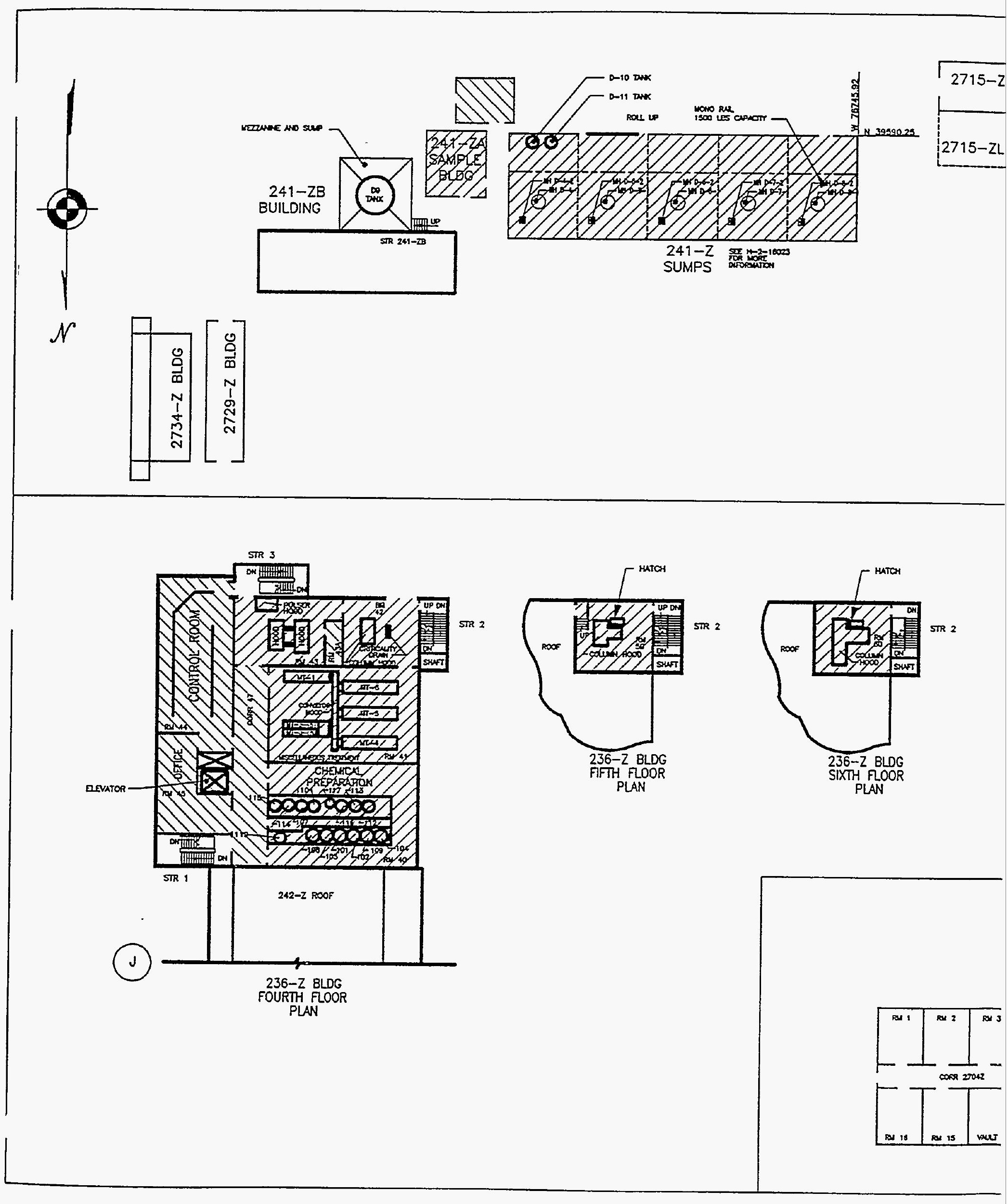




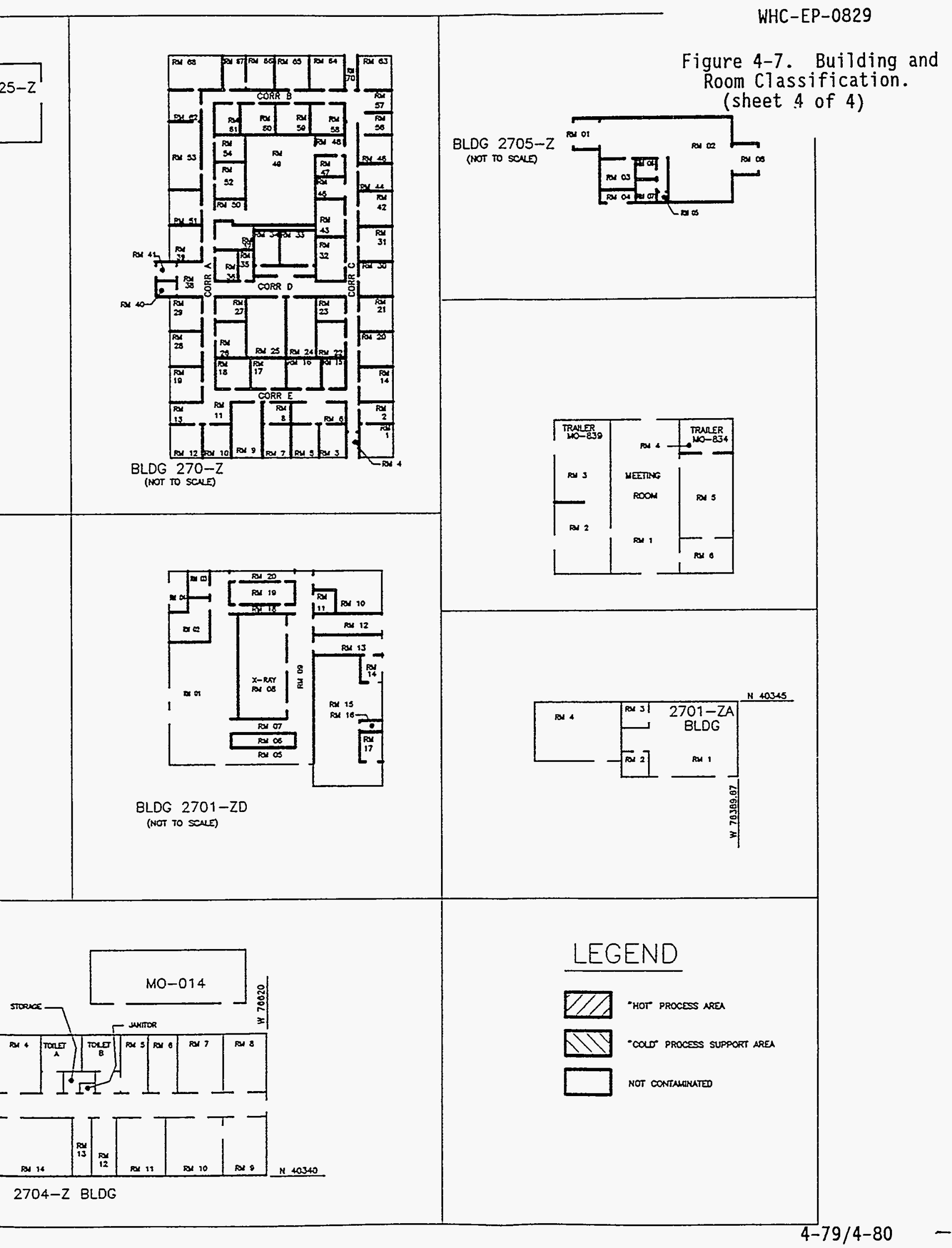


- Piping not in gloveboxes (i.e., waste drainage piping, service piping, and vacuum system piping).

- Ventilation system (i.e., filterboxes and filters, supply and exhaust duct work).

- Miscellaneous equipment that is not expected to be removed prior to D\&D (i.e., the mass spectrometer in the analytical lab).

Contaminated equipment encountered on drawings and during the walkdown which does not fit into any of the other categories is noted.

The piping and ventilation systems were considered on a building by building basis. Separate spreadsheets were developed to total volumes from drawings. All information not available from the documentation was gathered during the walkdown. The spreadsheets were then updated to reflect the walkdown data (see Appendices B and C).

Interviews with PFP personnel revealed other relevant drawings. These were used to update, verify and correct our data. The existence of gloveboxes and hoods was verified from drawings $\mathrm{H}-2-23400$ and $\mathrm{H}-2-29674$, which represent the status of 234-5Z and 236-Z, respectively, as of November 1993.

Specific assumptions, methods, and results are given in Section 5.0 by physical category. Totals for the PFP by waste type are provided in the Summary, Section 6.0. 
WHC-EP-0829

This page intentionally left blank. 
HHC-EP-0829

\subsection{RESULTS}

This section presents, by natural physical category, the solid waste volume estimates that are expected from the D\&D of the PFP. The uncertainties, global assumptions, and general approach for this project are stated in Section 4.0. The specific assumptions and methodology used to determine these volumes are detailed for each category of waste. Total D\&D waste estimates are given by waste type (DSW, LLW, LLMH, TRU, TRU MW) in Section 6.0.

\subsection{GLOVEBOXES AND HOODS}

Gloveboxes are used when handling of bare material is required. An operator can access material inside the glovebox through gloves installed in gloveports. The gloveports are strategically located to permit servicing of glovebox equipment. Operation and control of certain process equipment, valving, and material transfers are remote from the glovebox's interior, thereby minimizing extremity and whole-body exposures. Utilities provided to gloveboxes include electrical power, ventilation, fire protection, chemical lines, protected process water, nitrogen, and vacuum lines.

The metal shell of a typical glovebox is made of 4.8-mm (0.19-in.) 304-L stainless steel; the windows can be made of 1 aminated glass, leaded glass, or polycarbonate. Lead shielding is used for gloveboxes in many of the process areas to provide shielding from gamma radiation. Water wall barriers for neutron attenuation are used between RMC Line process areas and the control room and also around gloveboxes where neutron exposures may be high.

The normal ventilation system is designed to ensure control of airborne contaminants even if normal airflow patterns are disrupted, such as by an open glovebox access port. To ensure minimal escape of radioactive materials, the system also maintains airflows from lowest to highest contamination potential and filters all radioactive material work areas and confinement device exhausts. Supply airflow in radiation zones within process areas moves from ceiling diffusers to floor exhaust grills to maintain clean air in the breathing zone.

Gloveboxes may be equipped with internal fire protection equipment depending on need, which is determined by the nature of the glovebox operations and the associated fire loading. The internal automatic sprinkler system consists of sprinkler heads connected to pressurized water canisters. An electric switch monitors the system pressure and alarms at a facility fire panel and at the fire station when pressures drop below a set point. There are several alternatives to the internal sprinkler system. A fixed heat detector alarms at the plant as well as at the fire station. Magnesium oxide sand, available inside the glovebox, is used to extinguish small spot fires should they occur. A fire extinguisher and bayonet are kept outside the glovebox. In the event of a fire, the bayonet is punched through the glove and the contents of the extinguisher discharged. Metal gloveport covers are available to seal the damaged gloveport. 
The functions of a glovebox can require a supply of chemicals. Some are pumped in from chemical preparation tanks. Others are sealed into the glovebox's small plastic containers, or the containers are pushed through the glovebox sphincter.

Nitrogen gas is pumped into the gloveboxes from the building's supply system. Pressure inside the glovebox and the supply line pressure outside the glovebox are monitored. The suppiy valve automatically closes at a set differential pressure.

The vacuum source is either supplied by a self-contained vacuum pump or an external system. The vacuum is used to remove residual powders.

A standard laboratory hood is a stainless steel, open-faced fume hood that exhausts out the top. A pull-down front (sash) is provided. The front is constructed of glass, reinforced glass, or Plexiglas ${ }^{2}$ to protect the worker. The front of a fixed-faced hood is fixed in place, with open ports to provide access.

Many of the tanks used in glovebox processes are contained within the gloveboxes themselves. These interior tanks will be considered separately from those that are exterior to the gloveboxes (such as the chemical preparation tanks). Waste types are based on regulated chemicals expected to be left in the tanks as heels after flushing. The chemical contaminants possibly residing in tanks within gloveboxes are estimated based on the type of process occurring in the glovebox and the data available in the literature. Some tanks are partially enclosed by gloveboxes (such as the PRF pencil tanks). It is assumed that these must be removed from the glovebox during $D \& D$; therefore, these tanks will be considered external.

Most of the process piping and manifolding that services gloveboxes is contained within the gloveboxes themselves. For example, the PRF gloveboxes known as 1st East, 1st West, 2nd East, and 2nd West contain piping and manifolds for the pencil tanks and columns located in the canyon. This interior piping comprises a large portion of the volume of the glovebox and thus will be considered part of the glovebox rather than receiving separate consideration as piping. Piping that is exterior to the glovebox (such as that for flushed chemicals, process water, and vacuum systems) will be considered separately as piping in Section 5.3 .

Table 5-1 provides a list of PFP gloveboxes and hoods, their locations by building and room number, dimensions (including volumes), and, in cases in which it was known, the type of shielding used. The best known plutonium holdup estimate is also included by glovebox. For cases without data, a dash ("-") has been used. The total volume of solid waste expected from gloveboxes and hoods is $856 \mathrm{~m}^{3}\left(30,230 \mathrm{ft}^{3}\right)$, a 71 of which is expected to be TRU waste. A total of $34.9 \mathrm{~kg}$ ( $77 \mathrm{lb}$ ) of plutonium is estimated to be enclosed in the

2 Plexiglas is a trademark of Rohm \& Haas. 
Table 5-1. Gloveboxes and Hoods. (7 sheets)

\begin{tabular}{|c|c|c|c|c|c|c|c|c|c|}
\hline \multirow[t]{2}{*}{$\begin{array}{l}\text { Building } \\
\text { number }\end{array}$} & \multirow[t]{2}{*}{$\begin{array}{c}\text { Room } \\
\text { number }\end{array}$} & \multirow[t]{2}{*}{$\begin{array}{l}\text { Gloveboxes } \\
\text { and hoods }\end{array}$} & \multirow{2}{*}{$\begin{array}{l}\text { Type of } \\
\text { shielding }\end{array}$} & \multirow{2}{*}{$\begin{array}{l}\text { Plutonium } \\
\text { holdup } \\
\text { (g) }\end{array}$} & \multicolumn{3}{|c|}{$\begin{array}{c}\text { Dimensions } \\
(\mathrm{m})\end{array}$} & \multirow{2}{*}{$\begin{array}{c}\text { Volyme } \\
\left(\mathrm{m}^{3}\right)\end{array}$} & \multirow{2}{*}{$\begin{array}{c}\text { Information } \\
\text { origin }\end{array}$} \\
\hline & & & & & Length & Depth & Height & & \\
\hline \multirow{3}{*}{$234-52$} & \multirow{3}{*}{131} & GB-1-131 & -- & -- & 3.05 & 0.91 & 1.22 & 3.40 & Halkdown \\
\hline & & GB-2-131 & -- & -- & 3.05 & 0.91 & 1.22 & 3.40 & Malkdown \\
\hline & & GB-3-131 & -- & -- & 3.05 & 0.91 & 1.22 & 3.40 & Halkdown \\
\hline \multirow{4}{*}{$234-52$} & \multirow{4}{*}{134} & H-1-134 & -- & -- & 1.22 & 0.91 & 2.13 & 2.38 & Nalkdown \\
\hline & & H-2-134 & $\cdots$ & -- & 1.22 & 0.91 & 2.13 & 2.38 & Halkdown \\
\hline & & H-3-134 & $\cdots$ & $\cdots$ & 0.91 & 0.91 & 2.44 & 2.04 & Halkdown \\
\hline & & $H-4-134$ & -- & $\cdots$ & 0.91 & 0.91 & 2.44 & 2.04 & Halkdown \\
\hline \multirow{4}{*}{$234-52$} & \multirow{4}{*}{135} & $H-1-135$ & -- & -- & 1.22 & 0.91 & 2.13 & 2.38 & Halkdown \\
\hline & & H-2-135 & -- & -- & 1.22 & 0.91 & 2.13 & 2.38 & Halkdown \\
\hline & & H-3-135 & 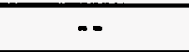 & 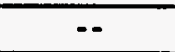 & 1.22 & 0.91 & 2.13 & 2.38 & Halkdown \\
\hline & & $\mathrm{H}-4-135$ & $\because$ & -- & 1.22 & 0.91 & 2.13 & 2.38 & Halkdown \\
\hline $234-52$ & 136 & $\begin{array}{c}\text { GB-1-136 (3 gloveboxes } \\
\text { together) }\end{array}$ & Water walls & $\cdots$ & 3.05 & 0.91 & 0.91 & 2.55 & Walkdown \\
\hline $234-52$ & 137 & Enissions spectroscopy & -- & -- & 2.44 & 1.22 & 0.91 & 2.72 & Halkdoun \\
\hline \multirow{6}{*}{$234-52$} & \multirow{6}{*}{139} & GB-1-139 & $\cdots$ & $\cdots$ & 0.91 & 0.91 & 3.05 & 2.55 & Halkdown \\
\hline & & GB-2-139 & $-\cdot$ & -- & 0.91 & 0.91 & 3.05 & 2.55 & Halkdown \\
\hline & & GB-3-139 & $\cdots$ & -- & 0.91 & 0.91 & 3.05 & 2.55 & Halkdown \\
\hline & & GB-4-139 & $\cdots$ & -- & 0.91 & 0.91 & 3.05 & 2.55 & Halkdown \\
\hline & & GB-5-139 & $\cdots$ & -- & 0.91 & 0.91 & 3.05 & 2.55 & Malkdown \\
\hline & & $\begin{array}{c}\text { 6B-6-139 (3 gloveboxes } \\
\text { together) }\end{array}$ & -. & -- & 1.83 & 0.91 & 0.91 & 1.53 & Halkdown \\
\hline \multirow{5}{*}{$234-52$} & \multirow{5}{*}{143} & $H-1-143$ & -- & $-\cdot$ & 1.22 & 0.91 & 2.13 & 2.38 & Halkdown \\
\hline & & $\mathrm{H}-2-143$ & $\cdots$ & -- & 1.22 & 0.91 & 2.13 & 2.38 & Walkdown \\
\hline & & $H-3-143$ & $\cdots$ & $\cdots$ & 1.22 & 0.91 & 2.13 & 2.38 & Malkdown \\
\hline & & $H-4-143$ & $-\cdot$ & $-\cdot$ & 1.22 & 0.91 & 2.13 & 2.38 & Halkdown \\
\hline & & $\mathrm{H}-5-143$ & -- & -- & 1.22 & 0.91 & 2.13 & 2.38 & Halkdown \\
\hline \multirow{2}{*}{$234-5 z$} & \multirow{2}{*}{144} & Hoods (8) & $\cdots$ & -- & 1.22 & 0.91 & 2.13 & 19.03 & Halkdown \\
\hline & & Ported hood & $\cdots$ & -- & 2.44 & 1.22 & 0.91 & 2.72 & Halkdown \\
\hline $234-52$ & 145 & GB-1-145 & -- & -. & 5.49 & 1.22 & 2.44 & 16.31 & Halkdown \\
\hline
\end{tabular}


Table 5-1. Gloveboxes and Hoods. (7 sheets)

\begin{tabular}{|c|c|c|c|c|c|c|c|c|c|}
\hline \multirow[t]{2}{*}{$\begin{array}{l}\text { Building } \\
\text { number }\end{array}$} & \multirow[t]{2}{*}{$\begin{array}{l}\text { Room } \\
\text { number }\end{array}$} & \multirow[t]{2}{*}{$\begin{array}{l}\text { Gloveboxes } \\
\text { and hoods }\end{array}$} & \multirow[t]{2}{*}{$\begin{array}{l}\text { Type of } \\
\text { shielding }\end{array}$} & \multirow{2}{*}{$\begin{array}{c}\text { Plutonium } \\
\text { holdup } \\
\text { (g) }\end{array}$} & \multicolumn{3}{|c|}{$\begin{array}{l}\text { Dimensions } \\
\text { (m) }\end{array}$} & \multirow[t]{2}{*}{$\begin{array}{l}\text { volume } \\
\left(m^{3}\right)\end{array}$} & \multirow[t]{2}{*}{$\begin{array}{c}\text { Information } \\
\text { origin }\end{array}$} \\
\hline & & & & & Length & Depth & Height & & \\
\hline \multirow{2}{*}{$234-52$} & \multirow{2}{*}{146} & Hoods (2) & $\cdots$ & $\cdots$ & 1.22 & 0.91 & 2.13 & 4.76 & Halkdown \\
\hline & & GB-5-146 & $\cdots$ & $\cdots$ & 2.44 & 1.22 & 1.22 & 3.62 & Halkdown \\
\hline \multirow{2}{*}{$234-52$} & \multirow{2}{*}{148} & $\mathrm{H}-1-148$ & $\cdots$ & - & 1.22 & 0.91 & 2.13 & 2.38 & Halkdown \\
\hline & & $H-1-148$ & $\cdots$ & - & 1.22 & 0.91 & 2.13 & 2.38 & Walkdown \\
\hline \multirow{2}{*}{$234-52$} & \multirow{2}{*}{149} & GB-2-149 & $\cdots$ & $\cdots$ & 1.52 & 1.22 & 0.91 & 1.70 & Halkdown \\
\hline & & $\mathrm{H}-1-149$ & $\cdots$ & $\cdots$ & 1.22 & 0.91 & 2.13 & 2.38 & Walkdown \\
\hline \multirow{2}{*}{$234-52$} & \multirow{2}{*}{152} & $G B-1-152$ & $\cdots$ & -- & 3.05 & 0.91 & 2.13 & 5.95 & Walkdown \\
\hline & & H-1-152 & $\cdots$ & $\cdots$ & 1.22 & 0.91 & 2.13 & 2.38 & Walkdown \\
\hline \multirow{4}{*}{$234-52$} & \multirow{4}{*}{153} & $H-1-153$ & $\cdots$ & -- & 1.22 & 0.91 & 2.13 & 2.38 & Halkdown \\
\hline & & $\mathrm{H}-2-153$ & $\cdots$ & $\cdots$ & 1.22 & 0.91 & 2.13 & 2.38 & Walkdown \\
\hline & & $\mathrm{H}-3-153$ & $\cdots$ & -- & 1.22 & 0.91 & 2.13 & 2.38 & Halkdown \\
\hline & & $H-4-153$ & $\cdots$ & $-\cdot$ & 1.22 & 0.91 & 2.13 & 2.38 & Walkdown \\
\hline \multirow{2}{*}{$234-52$} & \multirow{2}{*}{154} & $G B-1-154$ & -- & -- & 3.66 & 0.91 & 0.91 & 3.06 & Nalkdown \\
\hline & & ALK-1-154 & $-\cdot$ & $\cdots$ & 0.61 & 0.46 & 0.46 & 0.13 & Walkdown \\
\hline \multirow{4}{*}{$234-52$} & \multirow{4}{*}{155} & $H-1-155$ & $\cdots$ & $\cdots$ & 1.22 & 0.91 & 2.13 & 2.38 & Halkdown \\
\hline & & H-2-155 & $\cdots$ & -- & 1.22 & 0.91 & 2.13 & 2.38 & Walkdown \\
\hline & & $H-3-155$ & $-\cdot$ &.- & 1.22 & 0.91 & 2.13 & 2.38 & Walkdown \\
\hline & & $H-4-155$ & $\cdots$ & -- & 1.22 & 0.91 & 2.13 & 2.38 & Halkdown \\
\hline \multirow{4}{*}{$234-52$} & \multirow{4}{*}{156} & $H-1-156$ & $\cdots$ & $\cdots$ & 1.22 & 0.91 & 2.13 & 2.38 & Halkdown \\
\hline & & $H-2-156$ & $\cdots$ & $\cdots$ & 1.22 & 0.91 & 2.13 & 2.38 & Walkdown \\
\hline & & $\mathrm{H}-3-156$ & -- & $\cdots$ & 1.22 & 0.91 & 2.13 & 2.38 & Halkdown \\
\hline & & $H-4-156$ & $\cdots$ & $\cdots$ & 1.22 & 0.91 & 2.13 & 2.38 & Halkdown \\
\hline \multirow{4}{*}{$234-52$} & \multirow{4}{*}{157} & $G 8-3-157$ & -- & $\cdots$ & 1.22 & 0.91 & 2.13 & 2.38 & Halkdown \\
\hline & & GB-4-157 & $\cdots$ & $\cdots$ & 1.22 & 0.91 & 2.13 & 2.38 & Halkdown \\
\hline & & $H-1-157$ & $\because-$ & -- & 1.22 & 0.91 & 2.13 & 2.38 & Walkdown \\
\hline & & $\mathrm{H}-2-157$ & $\cdots$ & -- & 1.22 & 0.91 & 2.13 & 2.38 & Halkdown \\
\hline \multirow{2}{*}{$234-52$} & \multirow{2}{*}{159} & H-1-159 & $\cdots$ & $\cdots$ & 1.22 & 0.61 & 1.83 & 1.36 & Halkdown \\
\hline & & $H-2-159$ & $-\cdot$ & - & 1.22 & 0.61 & 1.83 & 1.36 & Nalkdown \\
\hline
\end{tabular}


Table 5-1. Gloveboxes and Hoods. (7 sheets)

\begin{tabular}{|c|c|c|c|c|c|c|c|c|c|}
\hline \multirow[t]{2}{*}{$\begin{array}{l}\text { Building } \\
\text { number }\end{array}$} & \multirow[t]{2}{*}{$\begin{array}{c}\text { Room } \\
\text { number }\end{array}$} & \multirow[t]{2}{*}{$\begin{array}{l}\text { Gloveboxes } \\
\text { and hoods }\end{array}$} & \multirow{2}{*}{$\begin{array}{c}\text { Type of } \\
\text { shielding }\end{array}$} & \multirow{2}{*}{$\begin{array}{l}\text { Plutonium } \\
\text { holdup } \\
\text { (g) }\end{array}$} & \multicolumn{3}{|c|}{$\begin{array}{c}\text { Dimensions } \\
(\mathrm{m})\end{array}$} & \multirow{2}{*}{$\begin{array}{l}\text { Volyme } \\
\left(m^{\prime}\right)\end{array}$} & \multirow{2}{*}{$\begin{array}{c}\text { Information } \\
\text { origin }\end{array}$} \\
\hline & & & & & Length & Depth & Height & & \\
\hline \multirow{4}{*}{$234-52$} & \multirow{4}{*}{166} & $\mathrm{HC}-4$ & $0.635-\mathrm{cm}\left(0.25-\mathrm{in}_{.}\right)$lead glass & 539 & 4.57 & 0.58 & 1.99 & 5.33 & Document \\
\hline & & HC- 6 & $\begin{array}{l}2.54-\mathrm{cm}(1-\mathrm{in} .) \text { lead glass; } \\
15.24-\mathrm{cm}(6-\mathrm{in.}) \text { lead/masonite } \\
\text { wall in front of tanks }\end{array}$ & 189 & 1.79 & 0.76 & 1.59 & 2.17 & Document \\
\hline & & $H-1-166$ & $\cdots$ &.- & 1.22 & 0.61 & 1.52 & 1.13 & Halkdown \\
\hline & & $H-2-166$ & -. & -- & 1.22 & 0.61 & 1.52 & 1.13 & Halkdown \\
\hline $234-52$ & 169 & $\mathrm{HA}-40 \mathrm{f}$ & $-\cdot$ & 190 & 3.96 & 1.52 & 1.22 & 7.36 & Walkdown \\
\hline \multirow[t]{2}{*}{$234-52$} & \multirow[t]{2}{*}{170} & $H C-46 f$ & $\begin{array}{c}1.27-\mathrm{cm}\left(0.5-\mathrm{in}_{.}\right) \text {lead, } 0.635-\mathrm{cm} \\
(0.25-\mathrm{in.}) \text { lead glass }\end{array}$ & 6 & 4.01 & 1.22 & 1.22 & 5.97 & $\begin{array}{l}\text { Halkdown } \\
\text { Drawings }\end{array}$ \\
\hline & & $\mathrm{H}-1-170$ & Lead glass & $-\cdot$ & 1.83 & 1.07 & 2.13 & 4.16 & Halkdown \\
\hline \multirow{9}{*}{$234-52$} & \multirow{9}{*}{179} & GB-179-1 & $\cdots$ & $\ldots$ & 1.52 & 1.22 & 2.44 & 4.53 & Halkdown \\
\hline & & GB-179-4 & $\cdots$ & 109 & 2.44 & 1.22 & 2.44 & 7.25 & Walkdown \\
\hline & & $G B-179-6$ & -- & 13 & 3.05 & 1.22 & 2.44 & 9.06 & Halkdown \\
\hline & & GB-179-9 & $\cdots$ & 34 & 3.05 & 1.22 & 2.44 & 9.06 & Halkdown \\
\hline & & $68-179-10$ & $\cdots$ & 19 & 1.22 & 1.22 & 2.44 & 3.62 & Halkdown \\
\hline & & $G B-179-12$ & -. & 33 & 1.22 & 1.22 & 2.44 & 3.62 & Halkdown \\
\hline & & H-179-2 & $\cdots$ & $\because$ & 0.61 & 1.22 & 2.44 & 1.81 & Walkdown \\
\hline & & $H-179-3$ & $\cdots$ &.- & 0.61 & 1.22 & 2.44 & 1.81 & Halkdown \\
\hline & & $H-179-11$ & $\cdots$ & $\cdots$ & 0.61 & 1.22 & 2.44 & 1.81 & Walkdown \\
\hline $234-52$ & 180 & $H-1-180$ & 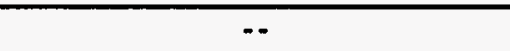 & -. & 1.22 & 0.91 & 0.91 & 1.02 & Walkdown \\
\hline $234-52$ & 183 & $G B-179-5$ & $\cdots$ & 4 & 2.44 & 0.61 & 2.13 & 3.17 & Halkdown \\
\hline \multirow{2}{*}{$234-52$} & \multirow{2}{*}{187} & Hoods, 3 together & $\cdots$ & 17 & 3.05 & 0.91 & 1.22 & 3.40 & Walkdown \\
\hline & & Hoods, 2 together & $\cdots$ & 16 & 3.05 & 0.91 & 1.22 & 3.40 & Halkdown \\
\hline $234-52$ & 188 & $G B-188-1$ & $\cdots$ & 26 & 4.57 & 1.22 & 1.83 & 10.19 & Halkdown \\
\hline \multirow{3}{*}{$234-52$} & \multirow{3}{*}{$221-\mathrm{C}$} & $G B-3-221 C$ & $\cdots$ & 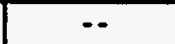 & 2.64 & 1.02 & 0.81 & 2.18 & $H-2-80208$ \\
\hline & & $\mathrm{H}-1-221 \mathrm{C}$ & $\cdots$ & $\cdots$ & 1.22 & 0.91 & 2.13 & 2.38 & Halkdown \\
\hline & & $\mathrm{H}-2-221 \mathrm{C}$ & $\cdots$ & $-\cdot$ & 1.22 & 0.91 & 2.13 & 2.38 & Halkdown \\
\hline \multirow{4}{*}{$234-52$} & \multirow{4}{*}{ 221-D } & $G B-5-2210$ & $\cdots$ & -- & 2.64 & 1.02 & 0.81 & 2.18 & $\mathrm{H}-2-80208$ \\
\hline & & $H-1-221 D$ & $\overline{-}$ & $\cdots$ & 1.22 & 0.91 & 2.13 & 2.38 & Walkdown \\
\hline & & $H-2-2210$ & -- & -- & 1.22 & 0.91 & 2.13 & 2.38 & Halkdown \\
\hline & & $H-3-2210$ & -. & - & 1.22 & 0.91 & 2.13 & 2.38 & Halkdown \\
\hline
\end{tabular}


Table 5-1. Gloveboxes and Hoods. (7 sheets)

\begin{tabular}{|c|c|c|c|c|c|c|c|c|c|}
\hline \multirow[t]{2}{*}{$\begin{array}{l}\text { Building } \\
\text { number }\end{array}$} & \multirow[t]{2}{*}{$\begin{array}{c}\text { Room } \\
\text { number }\end{array}$} & \multirow[t]{2}{*}{$\begin{array}{l}\text { Gloveboxes } \\
\text { and hoods }\end{array}$} & \multirow[t]{2}{*}{$\begin{array}{l}\text { Type of } \\
\text { shielding }\end{array}$} & \multirow{2}{*}{$\begin{array}{l}\text { Plutonium } \\
\text { holdup } \\
\text { (g) }\end{array}$} & \multicolumn{3}{|c|}{$\begin{array}{l}\text { Dimensions } \\
(\mathrm{m})\end{array}$} & \multirow[t]{2}{*}{$\begin{array}{l}\text { Volume } \\
\left(m^{m}\right)\end{array}$} & \multirow[t]{2}{*}{$\begin{array}{l}\text { Information } \\
\text { origin }\end{array}$} \\
\hline & & & & & Length & Depth & Height & & \\
\hline $234-52$ & $221-D$ & $H-4-2210$ & $-\cdots$ & -. & 1.22 & 0.91 & 2.13 & 2.38 & Halkdown \\
\hline \multirow{3}{*}{$234-52$} & \multirow{3}{*}{$221-E$} & $\mathrm{H}-1-221 \mathrm{E}$ & -- & $\cdots$ & 1.22 & 0.91 & 2.13 & 2.38 & Halkdown \\
\hline & & $\mathrm{H}-2-221 \mathrm{E}$ & -- & $\cdots$ & 1.22 & 0.91 & 2.13 & 2.38 & Walkdown \\
\hline & & $\mathrm{H}-3-221 \mathrm{E}$ & -- & $\cdots$ & 1.22 & 0.91 & 2.13 & 2.38 & Halkdown \\
\hline \multirow{2}{*}{$234-52$} & \multirow{2}{*}{227} & $H C-227-t$ & $2.54-\mathrm{cm}(1-\mathrm{in}$.$) lead$ & 2 & 3.66 & 0.61 & 4.57 & 10.19 & Document \\
\hline & & $H C-227-s$ & $\begin{array}{c}1.27-\mathrm{cm}(0.5 \text { in. }) \text { lead; } 0.635-\mathrm{cm} \\
(0.25-\mathrm{in.}) \text { lead }\end{array}$ & 529 & 3.66 & 0.61 & 3.51 & 7.82 & Document \\
\hline \multirow{5}{*}{$234-52$} & \multirow{5}{*}{$228-A$} & $H C-10 B$ & $2.54-\mathrm{cm}(1-$ in. $)$ lead glass & 37 & 0.70 & 0.48 & 0.69 & 0.23 & Document \\
\hline & & $\mathrm{HC}-7$ & $2.54-\mathrm{cm}(1-$ in. $)$ lead glass & 2,616 & 2.44 & 1.22 & 3.05 & 9.06 & Document \\
\hline & & $\mathrm{HC}-1$ & $2.54-\mathrm{cm}(1-$ in.) lead glass & 27 & 23.93 & 0.38 & 0.86 & 7.87 & Document \\
\hline & & $\mathrm{HC}-9 \mathrm{~b}$ & $\begin{array}{l}2.54-\mathrm{cm}(1-\mathrm{in} .) \text { lead gless and } \\
\text { water shield at mid-level }\end{array}$ & 5,595 & 2.29 & 0.86 & 4.34 & 8.57 & $\begin{array}{l}\text { Drawings } \\
\text { Document }\end{array}$ \\
\hline & & $\mathrm{HC}-11$ & $2.54-\mathrm{cm}\left(1-\mathrm{in}_{.}\right)$water walls & 202 & 2.15 & 0.70 & 0.69 & 1.03 & Document \\
\hline \multirow{5}{*}{$234-52$} & \multirow{5}{*}{$228 \cdot B$} & $\mathrm{HC}-1$ & $2.54-\mathrm{cm}(1-$ in. $)$ lead glass & $\cdots$ & 23.93 & 0.41 & 0.86 & 8.40 & Document \\
\hline & & $\mathrm{HC}-12 \mathrm{~s}$ & $2.54-\mathrm{cm}(1-\mathrm{in}$.$) lead glass$ & 73 & 0.81 & 0.71 & 1.09 & 0.63 & Document \\
\hline & & $H C-13 M D$ & $2.54-\mathrm{cm}(1-\mathrm{in}$.$) lead glass$ & 69 & 0.99 & 0.94 & 1.52 & 1.42 & Document \\
\hline & & HC- 16CC & $2.54-\mathrm{cm}(1-\mathrm{in}$.$) lead glass$ & 60 & 0.71 & 0.74 & 1.24 & 0.65 & Document \\
\hline & & $\mathrm{HC}-15 \mathrm{~A}, \mathrm{~B}, \mathrm{C}$ & $2.54-\mathrm{cm}\left(1-\mathrm{in}_{.}\right)$lead $\mathrm{glass}$ & 26 & 2.44 & 0.76 & 1.83 & 3.40 & Document \\
\hline \multirow{5}{*}{$234-52$} & \multirow{5}{*}{$228-\mathrm{C}$} & $\mathrm{HC}-1 \mathrm{TDC}$ & $2.54-\mathrm{cm}\left(1-\mathrm{in}_{.}\right)$lead glass & 48 & 1.19 & 0.76 & 1.40 & 1.27 & Document \\
\hline & & $H C-17 P$ & $2.54-\mathrm{cm}\left(1-\mathrm{in}_{.}\right)$lead glass & 50 & 0.91 & 0.76 & 1.22 & 0.85 & Document \\
\hline & & HC-18BS & $2.54-\mathrm{cm}(1-\mathrm{in}$.$) lead glass$ & 96 & 2.48 & 2.08 & 0.77 & 3.96 & Document \\
\hline & & $\mathrm{HC}-5$ & $\because$ & 324 & 1.04 & 0.61 & 0.96 & 0.61 & Halkdown \\
\hline & & $\mathrm{HC}-2$ & $\cdots$ & 155 & 15.54 & 0.40 & 0.96 & 5.93 & Halkdown \\
\hline $234-52$ & $230-A$ & $H C-21 C$ & $\begin{array}{c}1.27-\mathrm{cm}(0.5-\mathrm{in.}) \text { lead; } 0.635-\mathrm{cm} \\
(0.25-\mathrm{in.}) \text { lead glass } \\
\end{array}$ & 28 & 3.25 & 1.07 & 0.91 & 3.17 & Document \\
\hline $234-52$ & $230-8$ & $H C-21 A$ & $\begin{array}{c}1.27-\mathrm{cm}(0.5-\mathrm{in.}) \text { lead; } 0.635-\mathrm{cm} \\
(0.25-\mathrm{in.}) \text { lead glass }\end{array}$ & 147 & 3.25 & 1.07 & 0.91 & 3.17 & Document \\
\hline \multirow{3}{*}{$234-52$} & \multirow{3}{*}{$230-\mathrm{C}$} & HC- 60 & -- & 51 & 3.05 & 0.91 & 1.98 & 5.52 & Halkdown \\
\hline & & $\mathrm{HC}-3$ & $\cdots$ & 49 & 4.57 & 0.35 & 0.96 & 1.56 & Halkdown \\
\hline & & $\mathrm{HC}-4$ & $\cdots$ & -- & 2.74 & 0.76 & 2.13 & 4.46 & Halkdown \\
\hline $234-52$ & 232 & $H A-46$ & -- & -- & 5.33 & 0.53 & 4.19 & 11.92 & Walkdown \\
\hline $234-52$ & $235-A 1$ & $\mathrm{H}-14 \mathrm{CC}$ & $\cdots$ & 124 & 0.46 & 1.22 & 1.07 & 0.59 & Walkdown \\
\hline
\end{tabular}


Table 5-1. G7oveboxes and Hoods. (7 sheets)

\begin{tabular}{|c|c|c|c|c|c|c|c|c|c|}
\hline \multirow[t]{2}{*}{$\begin{array}{l}\text { Building } \\
\text { number }\end{array}$} & \multirow[t]{2}{*}{$\begin{array}{c}\text { Room } \\
\text { number }\end{array}$} & \multirow[t]{2}{*}{$\begin{array}{l}\text { Gloveboxes } \\
\text { and hoods }\end{array}$} & \multirow{2}{*}{$\begin{array}{l}\text { Type of } \\
\text { shielding }\end{array}$} & \multirow{2}{*}{$\begin{array}{l}\text { Plutonium } \\
\text { holdup } \\
\text { (g) }\end{array}$} & \multicolumn{3}{|c|}{$\begin{array}{l}\text { Dimensions } \\
\text { (m) }\end{array}$} & \multirow{2}{*}{$\begin{array}{l}\text { Volưme } \\
\left(m^{3}\right)\end{array}$} & \multirow{2}{*}{$\begin{array}{l}\text { Information } \\
\text { origin }\end{array}$} \\
\hline & & & & & Length & Depth & Height & & \\
\hline \multirow{7}{*}{$234-52$} & \multirow{7}{*}{ 235-A1 } & $H-14 D C$ & $\cdots$ & $\cdots$ & 1.22 & 0.91 & 1.22 & 1.36 & Walkdown \\
\hline & & $H-14 S$ & $\cdots$ & -- & 0.61 & 0.61 & 0.61 & 0.23 & Walkdown \\
\hline & & $\mathrm{H}-14 \mathrm{P}$ & $\cdots$ & -- & 1.22 & 1.22 & 1.83 & 2.72 & Malkdown \\
\hline & & $H-15$ & $\cdots$ & 175 & 0.91 & 0.30 & 3.66 & 1.02 & Walkdown \\
\hline & & $\mathrm{H}-16 \mathrm{CS}$ & $\cdots$ & -- & 1.52 & 1.52 & 0.76 & 1.77 & Halkdown \\
\hline & & $\mathrm{H}-16 \mathrm{BS}$ & $\cdots$ & 38 & 1.52 & 1.52 & 0.76 & 1.77 & Halkdown \\
\hline & & $\mathrm{H}-16 \mathrm{~L}$ & 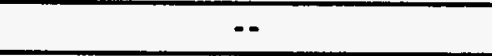 & $\cdots$ & 0.91 & 0.76 & 0.61 & 0.42 & Halkdown \\
\hline \multirow{7}{*}{$234-52$} & \multirow{7}{*}{ 235-A2 } & $\mathrm{H}-12 \mathrm{~S}$ & $\cdots$ & 19 & 0.61 & 1.22 & 0.61 & 0.45 & Halkdown \\
\hline & & H-13MD & $\cdots$ & 38 & 0.61 & 1.22 & 1.22 & 0.91 & Halkdown \\
\hline & & $H-13 G$ & $\cdots$ & 23 & 0.61 & 1.22 & 1.22 & 0.91 & Halkdown \\
\hline & & $H-13 A$ & $\cdots$ & 78 & 0.76 & 1.22 & 2.44 & 2.27 & Halkdown \\
\hline & & $H-13 B$ & -- & $\cdots$ & 0.76 & 1.22 & 2.44 & 2.27 & Halkdown \\
\hline & & $H-13 C$ & $\cdots$ & -- & 0.76 & 1.22 & 2.44 & 2.27 & Holkdown \\
\hline & & $\mathrm{HA}-13$ & $\cdots$ & $\cdots$ & 1.22 & 1.22 & 1.83 & 2.72 & Halkdown \\
\hline \multirow{9}{*}{$234-52$} & \multirow{9}{*}{$235-A 3$} & H-10 & $\cdots$ & 541 & 3.66 & 0.91 & 0.61 & 2.04 & Walkdown \\
\hline & & $H-8 A$ & $\cdots$ & -- & 4.27 & 1.07 & 0.91 & 4.16 & Walkdown \\
\hline & & $\mathrm{H}-11$ & $\cdots$ & 36 & 0.61 & 1.22 & 0.61 & 0.45 & Halkdown \\
\hline & & $H-7 A$ & $1.27-\mathrm{cm}(0.5-\mathrm{in}$.$) lead glass$ & 565 & 2.44 & 1.22 & 3.00 & 8.91 & Document \\
\hline & & $H-9 A$ & $1.27-\mathrm{cm}(0.5-$ in. $)$ lead glass & 2,821 & 2.29 & 0.86 & 3.76 & 7.42 & Document \\
\hline & & $H-98$ & $\cdots$ & $\cdots$ & 0.91 & 0.61 & 0.30 & 0.17 & Halkdown \\
\hline & & $H-9 C$ & $\overline{-}$ & $\cdots$ & 1.12 & 0.84 & 0.91 & 0.86 & Document \\
\hline & & $H-9 E$ & $\begin{array}{c}1.27-\mathrm{cm}(0.5-\mathrm{in} .) \text { lead; } 1.27-\mathrm{cm} \\
\left(0.5-\mathrm{in}_{.}\right) \text {lead glass }\end{array}$ & 11 & 1.63 & 0.91 & 0.71 & 1.06 & Document \\
\hline & & $H-90$ & $\cdots$ & 33 & 0.91 & 0.91 & 0.91 & 0.76 & Halkdown \\
\hline \multirow{6}{*}{$234-52$} & \multirow{6}{*}{$235-8$} & $H A-19 B 1$ & $\cdots$ & 45 & 0.46 & 0.40 & 0.99 & 0.18 & Drawings \\
\hline & & $\mathrm{HA}-19 \mathrm{~B} 2$ & $\cdots$ & $\cdots$ & 1.52 & 0.76 & 0.76 & 0.88 & Walkdown \\
\hline & & HA-19 & $\because$ & $\cdots$ & 4.57 & 0.46 & 1.22 & 2.55 & Walkdown \\
\hline & & HC-235-B-1 & $\cdots$ & -- & 5.49 & 0.91 & 2.74 & 13.76 & Halkdown \\
\hline & & HC-235-B-2 & $\cdots$ & 1 & 3.05 & 0.76 & 1.52 & 3.54 & Halkdown \\
\hline & & HA-22B & $\overline{-}$ & 29 & 1.07 & 0.76 & 2.44 & 1.98 & Malkdown \\
\hline
\end{tabular}


Table 5-1. Gloveboxes and Hoods. (7 sheets)

\begin{tabular}{|c|c|c|c|c|c|c|c|c|c|}
\hline \multirow[t]{2}{*}{$\begin{array}{l}\text { Building } \\
\text { number }\end{array}$} & \multirow[t]{2}{*}{$\begin{array}{l}\text { Room } \\
\text { number }\end{array}$} & \multirow{2}{*}{$\begin{array}{l}\text { Gloveboxes } \\
\text { and hoods }\end{array}$} & \multirow{2}{*}{$\begin{array}{l}\text { Type of } \\
\text { shielding }\end{array}$} & \multirow{2}{*}{$\begin{array}{c}\text { Plutonium } \\
\text { holdup } \\
\text { (g) }\end{array}$} & \multicolumn{3}{|c|}{$\begin{array}{l}\text { Dimensions } \\
\text { (m) }\end{array}$} & \multirow{2}{*}{$\begin{array}{l}\text { Volyme } \\
\left(m^{3}\right)\end{array}$} & \multirow[t]{2}{*}{$\begin{array}{l}\text { Information } \\
\text { origin }\end{array}$} \\
\hline & & & & & Length & Depth & Height & & \\
\hline \multirow{4}{*}{$\begin{array}{l}234-52 \\
\text { (cont.) }\end{array}$} & \multirow{4}{*}{$\begin{array}{l}235-B \\
(\text { cont.) }\end{array}$} & $H A-21 \quad I$ & $\begin{array}{c}1.27-\mathrm{cm}(0.5-\mathrm{in} .) \text { lead; } 0.635-\mathrm{cm} \\
(0.25-\text { in. }) \text { lead glass }\end{array}$ & 460 & 3.66 & 1.07 & 1.22 & 4.76 & Halkdown \\
\hline & & HA-2OMB & $\begin{array}{c}1.27-\mathrm{cm}(0.5-\mathrm{in} .) \text { lead; } 0.635-\mathrm{cm} \\
(0.25-\text { in. }) \text { lead glass }\end{array}$ & 275 & 4.57 & 1.07 & 1.22 & 5.95 & Walkdown \\
\hline & & $\mathrm{HA-28}$ & $\cdots$ & 89 & 18.29 & 0.91 & 0.46 & 7.65 & Halkdown \\
\hline & & $H A-23 S$ & $\begin{array}{c}1.27-\mathrm{cm}(0.5-\mathrm{in} .) \text { lead, } 0.635-\mathrm{cm} \\
(0.25-\mathrm{in.}) \text { lead glass, water walls }\end{array}$ & 59 & 9.75 & 3.05 & 3.66 & 108.74 & Halkdown \\
\hline \multirow{4}{*}{$234-52$} & \multirow{4}{*}{$235-0$} & Hood 1008 & -- & $\cdots$ & 3.66 & 5.49 & 0.91 & 18.35 & Halkdown \\
\hline & & Hood 200 & -- & $\cdots$ & 3.96 & 2.44 & 1.52 & 14.72 & Halkdown \\
\hline & & Hood 300B & $\cdots$ & $\cdots$ & 4.57 & 1.22 & 1.22 & 6.80 & Halkdown \\
\hline & & Hood 400 & $\cdots$ & $\cdots$ & 2.13 & 0.91 & 1.52 & 2.97 & Halkdown \\
\hline \multirow[t]{2}{*}{$236-2$} & \multirow[t]{2}{*}{11} & $\begin{array}{c}\text { Corridor glovebox (1st } \\
\text { Hest) }\end{array}$ & $\cdots$ & 3,787 & 17.68 & 0.61 & 2.44 & 26.28 & Halkdown \\
\hline & & Corridor glovebox & 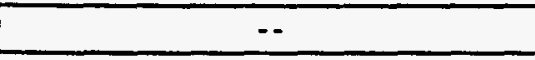 & $-\cdot$ & 1.83 & 0.61 & 0.91 & 1.02 & Halkdown \\
\hline \multirow[t]{2}{*}{$236-2$} & \multirow[t]{2}{*}{14} & $\begin{array}{c}\text { Corridor glovebox (1st } \\
\text { East) }\end{array}$ & - & 4,970 & 17.68 & 0.61 & 2.44 & 26.28 & Halkdown \\
\hline & & Corridor glovebox & $\cdots$ & $\cdots$ & 1.83 & 0.61 & 0.91 & 1.02 & Halkdown \\
\hline $236-2$ & 21 & $\begin{array}{c}\text { Corridor glovebox (2nd } \\
\text { West) }\end{array}$ & $\cdots$ & 2,912 & 19.51 & 0.61 & 2.44 & 29.00 & Halkdown \\
\hline $236-2$ & 25 & $\begin{array}{c}\text { pH glovebox, corridor (2nd } \\
\text { East) }\end{array}$ & $\cdots$ & 2,998 & 19.51 & 0.61 & 2.44 & 29.00 & Walkdown \\
\hline $236-2$ & 27 & Maintenance glovebox & No lead & 73 & 3.05 & 1.22 & 0.91 & 3.40 & Halkdown \\
\hline \multirow{6}{*}{$236-2$} & \multirow{6}{*}{41} & MT-6 & $\cdots$ & 653 & 4.88 & 0.76 & 2.39 & 8.87 & Drawings \\
\hline & & MT -5 & $\cdots$ & 394 & 5.33 & 0.99 & 1.68 & 8.86 & Drawings \\
\hline & & $M T-4$ & $\because$ & 357 & 5.49 & 0.91 & 2.44 & 12.23 & $\mathrm{H}-2-29674$ \\
\hline & & $M T-3$ & -- & 656 & 3.35 & 1.07 & 2.44 & 8.72 & H-2-29674 \\
\hline & & $\mathrm{HT}-1$ & $\cdots$ & 221 & 3.05 & 0.76 & 1.52 & 3.54 & $\mathrm{H}-2-29674$ \\
\hline & & Transfer glovebox & $\cdots$ & 369 & 6.40 & 0.46 & 0.91 & 2.68 & Halkdown \\
\hline $236-2$ & 42 & Colum glovebox & $-\cdot$ & 280 & 1.83 & 1.83 & 2.44 & 8.16 & Walkdown \\
\hline \multirow{4}{*}{$236-2$} & \multirow{4}{*}{43} & Dissolver canning facility & $\cdots$ & $\cdots$ & 1.65 & 0.56 & 0.91 & 0.84 & Drawings \\
\hline & & Dissolver loading glovebox & $\because$ & $\cdots$ & 1.65 & 0.56 & 0.79 & 0.73 & Drauings \\
\hline & & Pulser glovebox & $\cdots$ & $\because$ & 0.91 & 0.61 & 0.91 & 0.51 & Walkdown \\
\hline & & Charging glovebox & Lead Hindows & $\cdots$ & 1.52 & 0.61 & 0.91 & 0.85 & Halkdown \\
\hline
\end{tabular}




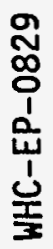

\begin{tabular}{|c|c|c|c|c|c|c|c|c|c|}
\hline & $c^{111} 21 \cdot 958$ & : sunjo & 18701 & & $206^{\prime} \pm E$ & :dnpjoy un!uoznjd jez01 & & & \\
\hline S6U!MEJO & $2 \varepsilon^{\circ} 0$ & $97^{\circ} 0$ & $9 L \cdot 0$ & $16^{\circ} 0$ & $b$ & $-\cdot$ & p0o4 7no-p807 & \multirow{2}{*}{$9 \varepsilon 9$} & \multirow{2}{*}{$8 z-9 \varepsilon<2$} \\
\hline S6u!me $d 0$ & $52 \cdot 0$ & $97 \cdot 0$ & $9 L^{\circ} 0$ & $1 L^{\circ} 0$ & $\cdots$ & $\cdots$ & poo4 u!-p807 & & \\
\hline s6u!neda & $15 \cdot 1$ & $25 * 1$ & $29 \cdot 1$ & $19^{\circ} 0$ & - & - & s-1n & \multirow{5}{*}{ •Бр18 } & \multirow{5}{*}{$z-272$} \\
\hline S6U!MBJo & $\varepsilon \Sigma^{\circ} 0$ & 76.0 & $\varepsilon 5^{\circ} 0$ & $99^{\circ} 0$ & $\cdots$ & $\because$ & $7-14$ & & \\
\hline SEU!ME.JO & 56.8 & $6 \varepsilon^{27}$ & $\varepsilon S^{\circ} 0$ & $18^{\circ} \varepsilon$ & -- & -- & $\varepsilon-1 n$ & & \\
\hline sGu!neJa & 76.2 & $\$ t^{\circ} 2$ & $19 \cdot 0$ & 86.1 & $\cdots$ & -- & $2-14$ & & \\
\hline S6U!MEدa & $8 \varepsilon^{\circ} \angle l$ & $62 * 2$ & $\$ 8 \% 0$ & $20^{\circ} 6$ & -- & $\cdots$ & $1-1 \mathrm{M}$ & & \\
\hline иморуіви & $89^{\circ} 0$ & $16 \cdot 0$ & $19^{\circ} 0$ & $22 \cdot 1$ & $\because$ & - & $\forall z-1+2-1-89$ & \multirow{2}{*}{-5p18 } & \multirow{2}{*}{$v z-\operatorname{tyz}$} \\
\hline uморх1ен & $80^{\circ} 0$ & $97^{\circ} 0$ & $0 \varepsilon^{\circ} 0$ & $19^{\circ} 0$ & $\cdots$ & -- & $v z-172-2-89$ & & \\
\hline uморх18n & $98^{\circ} \mathrm{sl}$ & $77^{2} 2$ & $\varepsilon l \cdot 2$ & $50^{\circ} \varepsilon$ & 612 & $\cdots$ & 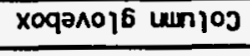 & 09 & $Z-9 \varepsilon 2$ \\
\hline инору|вн & $9 L^{-8} 8$ & $7+2$ & $\varepsilon 8.1$ & 28.1 & $2<1$ & -- & xоqวл0,6 umloj & os & $2-9 \varepsilon 2$ \\
\hline umopxign & 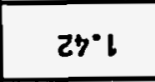 & $9 L^{\circ} 0$ & 19.0 & $50^{\bullet} \varepsilon$ & 9 & $\cdots$ & бu!prol Gu!uuBJ & $\begin{array}{c}(-24000) \\
\sum 7\end{array}$ & $\begin{array}{c}(-24005) \\
z-952\end{array}$ \\
\hline \multirow{2}{*}{$\begin{array}{c}4 ! 5 ! j o \\
\text { uo!?ewisojul }\end{array}$} & \multirow{2}{*}{$\begin{array}{l}\left({ }^{(11)}\right) \\
\operatorname{sun} \mid{ }_{1}\end{array}$} & $346 !$ 애 & प7dad & 476407 & \multirow{2}{*}{$\begin{array}{c}\text { (B) } \\
\text { dnplou } \\
\text { un!uozn\}d }\end{array}$} & \multirow{2}{*}{$\begin{array}{l}\text { 6u!plo!yus } \\
\text { to ədk1 }\end{array}$} & \multirow{2}{*}{ 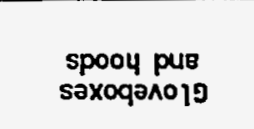 } & \multirow[b]{2}{*}{$\begin{array}{l}\text { Jaqunu } \\
\text { wooy }\end{array}$} & \multirow[b]{2}{*}{$\begin{array}{l}\text { Jequnu } \\
\text { Gu!pl!ng }\end{array}$} \\
\hline & & & $\begin{array}{c}\text { (U) } \\
\text { suo! surul }\end{array}$ & & & & & & \\
\hline
\end{tabular}


gloveboxes and hoods. Dimensions are from documents, drawings, and walkdown estimates (determined visually to the nearest three decimeters [foot]), as noted. This table was verified and corrected during the walkdown. The existence of gloveboxes and hoods was verified from drawings $\mathrm{H}-2-23400$ and $\mathrm{H}-2-29674$, which represent the status of 234-5Z as of November 1993 and 236-Z as of February 1994, respectively.

The Division of Military Application Line was used from 1945 to 1965 for the fabrication, inspection, and packaging of plutonium weapon components. Most of the Division of Military Application equipment was removed during the decommissioning activities that occurred between May 28, 1974, and May 25, 1976. The equipment removed included most of the gloveboxes, but some remained to perform additional functions. The estimates presented in this study do not include the waste removed by prior activities. It is interesting to note, however, that the Division of Military Application equipment that was removed is stored in 61 boxes located in burial ground 218-W-3A, trench 17 . These boxes have a volume of $2,231 \mathrm{~m}^{3}\left(78,781 \mathrm{ft}^{3}\right)$, a net weight of $149,209 \mathrm{~kg}$ $(328,950 \mathrm{lb})$, and contain $5,591 \mathrm{~g}(12.33 \mathrm{lb})$ of plutonium (Demiter 1991).

Knowledge of the type of shielding is useful because lead, a common shielding material, is a hazardous material regulated by WAC 173-303-040. In some cases, water-filled walls are used as shielding, which may pose unique challenges for D\&D and disposal.

Table 5-2 is a list of tanks that are internal to gloveboxes. The 1ist includes the building, location within the building, size, volume, contents, and classification for each tank identified in the available literature. It should be noted that this list includes previously documented data and is most likely not inclusive of all tanks within gloveboxes.

Tank dimensions and contents listed in Table 5-2 have been accumulated from available literature, operating processes, and walkdown data. Information that was not found is indicated as unknown in the table and unknown volumes were assumed to be zero. Both the classification and volume of the tanks assume that residuals of chemicals once contained in the tanks are still present. This assumption results in a conservative estimate; however, most process gloveboxes will be considered TRU waste, in which case the internal tanks would also most likely be equally contaminated. Therefore, virtually 100 percent of the solid waste expected from the D\&D of tanks internal to gloveboxes is classified as TRU or TRU MW.

Based on the information available, it is estimated that the total volume of solid waste expected from internal glovebox tanks is $1.73 \mathrm{~m}^{3}$. This total volume is composed of approximately $1.717 \mathrm{~m}^{3}$ of TRU MW and 0.017 of TRU waste. Thus, TRU MW represents approximately 99 percent of the solid waste expected to be generated by tanks internal to gloveboxes. 
Table 5-2. Tanks Internal to Gloveboxes. (4 sheets)

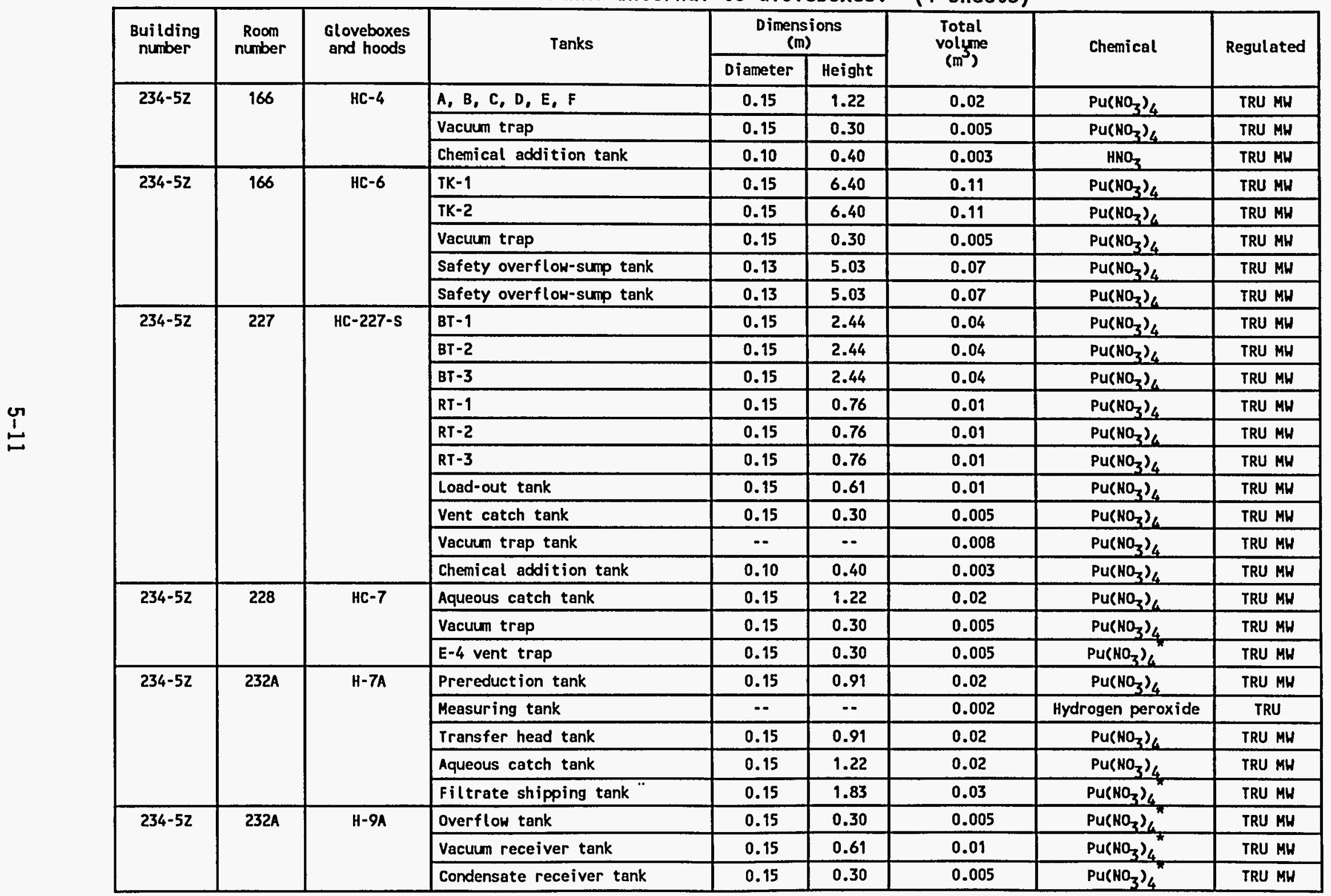


Table 5-2. Tanks Internal to Gloveboxes. (4 sheets)

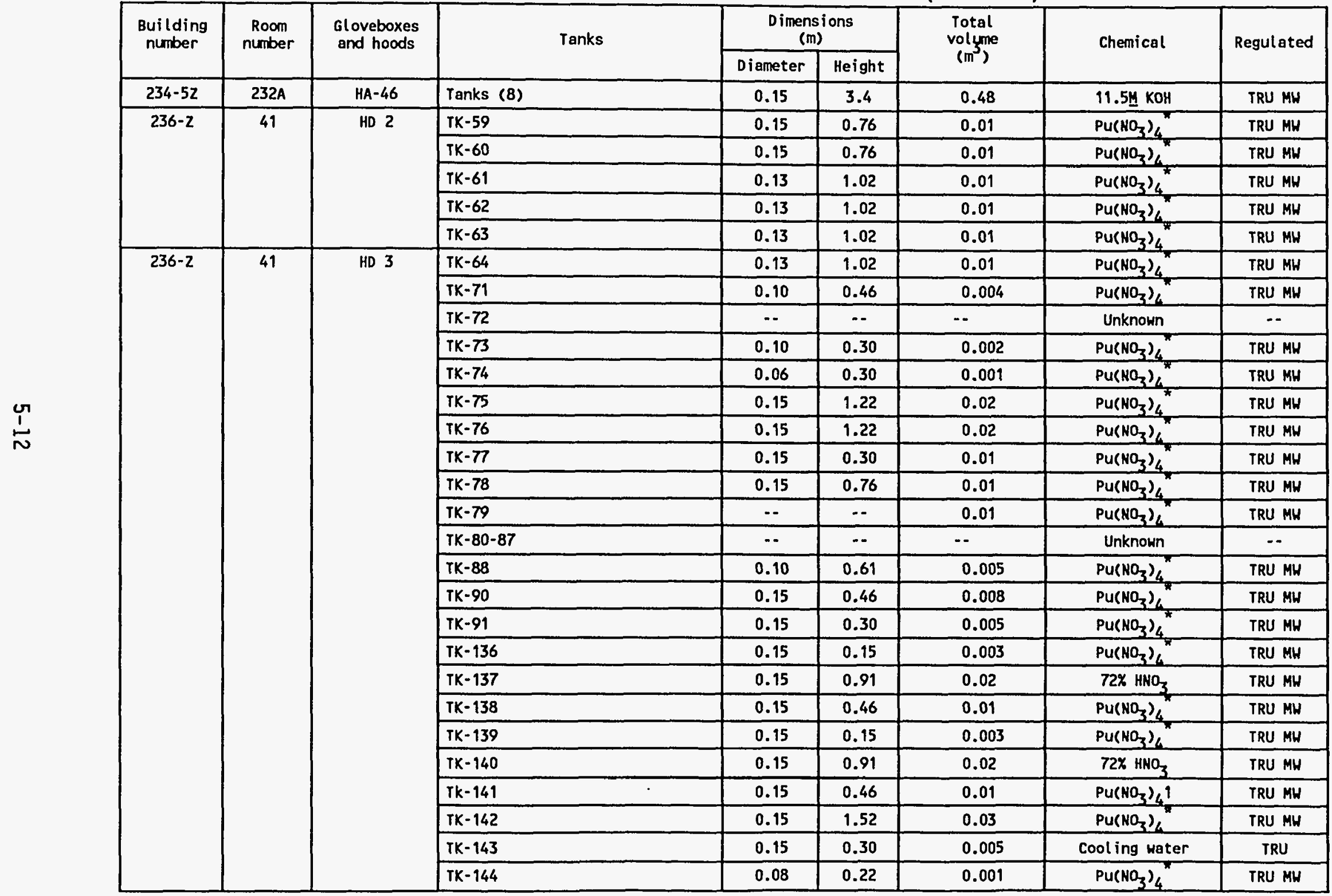


Table 5-2. Tanks Internal to Gloveboxes. (4 sheets)

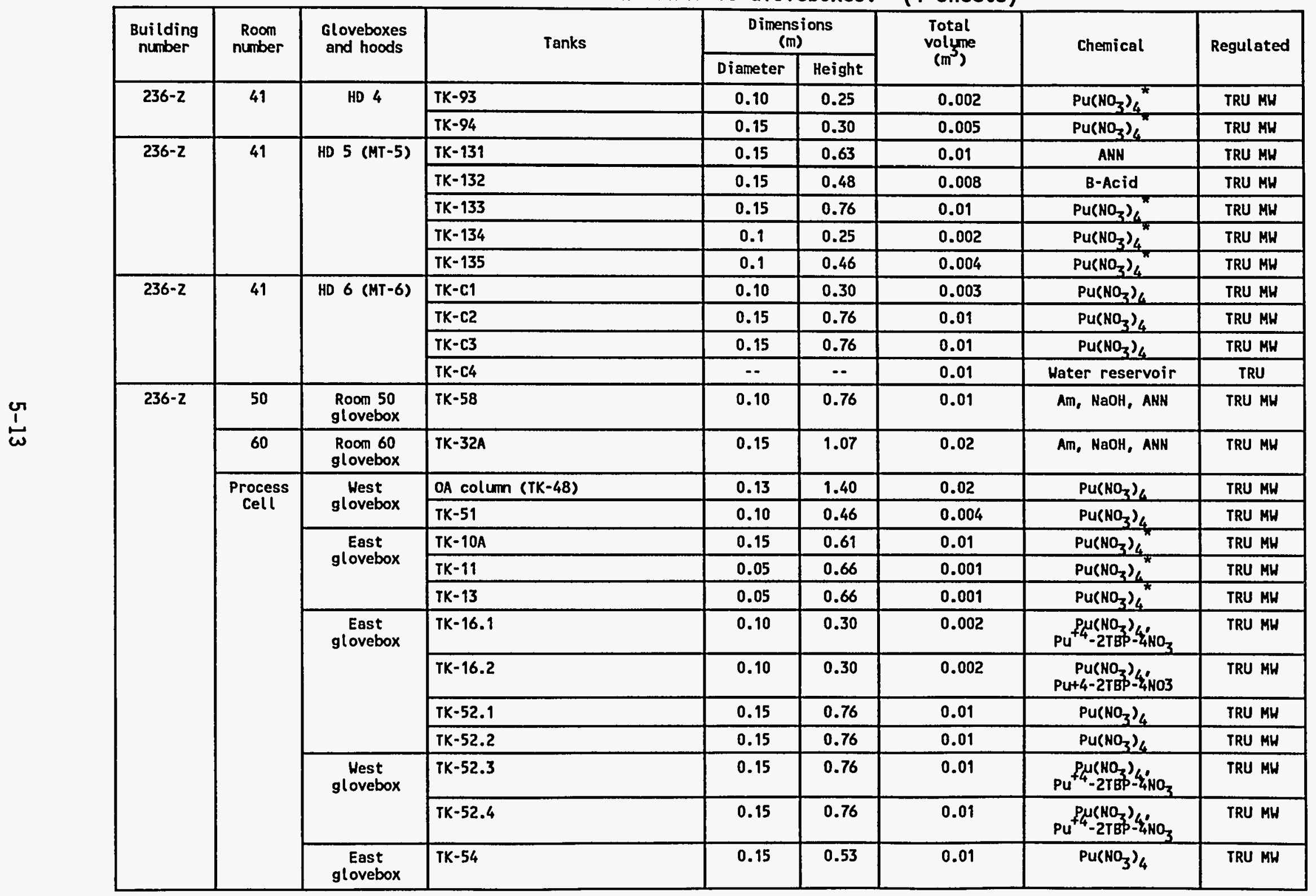


Table 5-2. Tanks Internal to Gloveboxes. (4 sheets)

\begin{tabular}{|c|c|c|c|c|c|c|c|c|}
\hline \multirow[t]{2}{*}{$\begin{array}{l}\text { Building } \\
\text { number }\end{array}$} & \multirow[t]{2}{*}{$\begin{array}{c}\text { Room } \\
\text { number }\end{array}$} & \multirow[t]{2}{*}{$\begin{array}{l}\text { Gloveboxes } \\
\text { and hoods }\end{array}$} & \multirow[t]{2}{*}{ Tanks } & \multicolumn{2}{|c|}{$\begin{array}{l}\text { Dimensions } \\
\text { (m) }\end{array}$} & \multirow{2}{*}{$\begin{array}{l}\text { Total } \\
\text { volugme } \\
\left(m^{3}\right)\end{array}$} & \multirow[t]{2}{*}{ Chemical } & \multirow[t]{2}{*}{ Regulated } \\
\hline & & & & Diameter & Height & & & \\
\hline \multirow[t]{2}{*}{$242-2$} & \multirow[t]{2}{*}{ Unknown } & \multirow[t]{2}{*}{$H T-1$} & TK-DH-5 & 0.10 & 0.66 & 0.01 & $\begin{array}{c}\mathrm{Pu}, \mathrm{Am}, \mathrm{HNO3}, \\
\text { DBBP-CCL4 }\end{array}$ & TRU MH \\
\hline & & & TK-DW-51 & 0.10 & 0.79 & 0.01 & $\begin{array}{c}\mathrm{Pu}_{1} \mathrm{Am}_{1} \mathrm{HNO}_{3} \\
\mathrm{DBBP}-\mathrm{CCl}_{4}\end{array}$ & TRU MH \\
\hline \multirow[t]{3}{*}{$242-2$} & \multirow[t]{3}{*}{ Unknown } & \multirow[t]{3}{*}{ UT -2 } & $T K-W-14$ & 0.17 & 0.67 & 0.02 & $\begin{array}{l}\text { Am, } \mathrm{HNO}_{3} \\
\mathrm{DBBP}-\mathrm{CCC}_{4}\end{array}$ & TRU MH \\
\hline & & & $T K-H-23$ & 0.15 & 0.61 & 0.01 & $\begin{array}{l}\mathrm{Am}, \mathrm{HNO}_{3}, \\
\mathrm{DBBP}-\mathrm{CCl}_{4}\end{array}$ & TRU MH \\
\hline & & & $T K-D W-13$ & 0.10 & 0.66 & 0.01 & 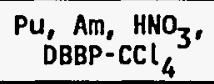 & TRU MH \\
\hline & & & & \multicolumn{2}{|c|}{$\begin{array}{l}\text { Total volume: } \\
\text { TRU volume: } \\
\text { TRU MW volume: }\end{array}$} & $\begin{array}{l}1.73 \mathrm{~m}_{3}^{3} \\
0.017 \mathrm{~m}^{3} \\
1.717 \mathrm{~m}^{2}\end{array}$ & & \\
\hline
\end{tabular}

"Estimate based on process information.

ANN = Aluminum ni trate nanohydrate.

B acid $=12 \mathrm{M} \mathrm{HNO}+0.35 \mathrm{M} \mathrm{HF}$.

CCl $=$ Carbon tetrachloride.

DBBP = Dibutylbiphenyl.

$H F=$ Hydrofluoric acid.

$H N=$ Hydroxylamine nitrate.

$\mathrm{HNO}_{3}=\mathrm{Nitric}$ acid.

$\mathrm{H}_{2} \mathrm{O}_{2}=$ Hydrogen peroxide.

HS = Hydroxylamine sulfate.

$\mathrm{MnO}_{\mathrm{K}}=$ Potassium permanganate

KOH = Potassium hydroxide.

$\mathrm{Na}_{2} \mathrm{CO}_{3}=$ Sodium carbonate.

NâNO = Sodium nitrate.

NaOH = Sodium hydroxide.

$\mathrm{Pu}=$ Plutoniun.

Pu(NO $)_{3}=$ Plutonium nitrate.

TBP = Tributyl phosphate.

TRU MH = Transuranic mixed Maste

$U=$ Uranium. 
WHC-EP-0829

\subsection{TANKS CONSIDERED EXTERNAL TO GLOVEBOXES OR HOODS}

During plutonium processing, several tanks were required to supply process chemicals, receive process chemicals, mix chemicals, and catch overflow. Typical storage tanks have inlet and outlet control valves, a pumpoperated recirculation line used to keep the tanks mixed and to prevent solids from settling out, a pump bypass 7 ine, a sampling valve, a drain valve, and a transfer control valve. The PFP process tanks are typically constructed of stainless steel; however, there are glasș, polyvinyl chloride, and titanium tanks. Some tanks are lined with Teflon ${ }^{3}$, and column tanks usually contain mixing media, chemical exchange media, or filter media.

The functions of the tanks include extracting plutonium from aqueous feed, stripping plutonium from organic feed, plutonium scrubbing, uranium scrubbing, dibutyl phosphate scrubbing, and organic reacidification.

The PRF canyon pencil tanks, TK-32, TK-33, TK-WS-1, TK-123, TK-69, and TK-48 are al so known as columns CA, CC, CO, CU, CX, and $O A$, respectively. They range in height from 1.2 to $15.5 \mathrm{~m}$ ( 4 to $51 \mathrm{ft}$ ) and extend through as many as six floors of the 236-Z Building. Each has a bottom disengaging section and a top disengaging section of either Pyrex ${ }^{4}$ glass or stainless steel. The remaining length of the column is made of 304-L stainless steel tubing, or Teflon-lined stainless steel pipe. Some also include a packed section containing Teflon Raschig rings, Kynar ${ }^{5}$ Raschig rings, or Kynar saddles. Three of the columns are pulsed by air pulsers in which air pressure is alternately applied and vented on a 25.4-mm (1-in.) pulse leg connected to the bottom of the column and extending up into a higher floor's glovebox. As stated in the previous section, these columns will be considered external because they are only partially enclosed by gloveboxes and will most likely be removed and separated from the gloveboxes.

Facilities in rooms 336 and 337, Building 234-5Z, are used to prepare and supply chemicals to the RMC Line. Tanks are arranged along the south wall of the rooms in a low-walled sump. Room 336, containing tanks C, D-1, D-2, G-1, $\mathrm{G}-2$, and $\mathrm{E}-1$, is hooded and ventilated through roof exhausts. The room is equipped with safety showers, floor drains, and fire sprinklers. The walls and ceiling are constructed of 19-mm (0.75-in.) plaster on metal lath. Motor controls for outside chemical pumps are located in room 337.

Chemical-makeup vessels are also located in room 40 of Building 236-Z. This room contains the tanks, scales, and associated equipment used in preparing the organic solvent and other chemicals that were used in the solvent extraction and other processing activities in 236-Z. A11 makeup vessels are located within a dike area for spill control and empty to a catch tank for collection. The dike area accommodates the contents of the largest vessel and is alarmed for spill detection. The area outside of the dike is manned during the makeup operation.

3 Teflon is a trademark of E. I. du Pont de Nemours \& Company.

${ }^{4}$ Pyrex is a trademark of Corning Glass Works.

${ }^{5}$ Kynar is a trademark of the Pennwalt Corporation. 
Building 241-Z covers a below grade liquid waste collection system containing five tanks in five, separate, covered cells. Each tank is made of stainless steel, and each has an approximate volume of $18 \mathrm{~m}^{3}\left(628 \mathrm{ft}^{3}\right)$. Nuclear criticality safety for these tanks is provided administratively by limiting plutonium concentrations and quantities. This radioactive liquid waste facility is used for intermediate storage and neutralization of PFP aqueous wastes and is active. After neutralization, the wastes are pumped to the 244-TX Tank Farm.

Table 5-3 provides a list of potentially contaminated tanks that are considered external to gloveboxes or hoods, their locations by building and room number, dimensions (including volume), and expected solid waste classifications. The total volume of solid waste expected from the D\&D of external tanks $i$ isted in the table is $173 \mathrm{~m}^{3}$. The table was generated based on data from available Titerature. Any additional dimensions resulting from the walkdown were estimated visually to the nearest three decimeters (foot). Walkdown information is indicated in the table with an asterisk. of the total expected solid waste volume, $133 \mathrm{~m}^{3}$ is estimated to be TRU MW and $40.3 \mathrm{~m}^{3}$ DSW.

The classification of external tanks results from process information contained in available documents. Information that was not found is indicated as unknown in the table and unknown volumes were assumed to be zero. If the chemical contents for a particular tank were not documented, process information was used to estimate the possible classification of the expected solid waste. It should be noted that the external tanks 1 isted in Table 5-3 are not inclusive of all tanks in the PFP; the table only included tanks 1 isted in the literature and tanks identified during the walkdown.

\subsection{PIPING NOT IN GLOVEBOXES OR HOODS}

Before January 1994, liquid waste streams, including condenser cooling water from gloveboxes, were collected from PFP facilities via an extensive drainage system and then routed to the vitreous clay pipe, Z-20 crib stream system. These waste streams were normally free of radioactive contamination, but they had the potential of becoming contaminated. Each of the waste streams from various PFP facilities was routed to a specific drain header and discharged into the $Z-20 \mathrm{crib}$ stream at a specific manhole. The waste collection lines were sloped to provide gravity-flow to the Z-20 crib stream. An exception is the waste collection 1 ine in the $291-Z$ waste sump; this 7 ine was pumped. Surface water runoff, resulting from rain and snow melt, was collected by four storm sewer drains located south of the 234-5Z Building. This water was also routed to the Z-20 crib stream.

The various manholes in the $\mathrm{Z}-20$ crib stream system provided access for flushing and/or cleanout of the $Z-20$ crib stream. The manholes also provided access for grab sampling, when required, to determine the origin of abnormal chemical or radioactive contamination.

The Z-20 crib sewer stream effluent was disposed to the ground via the Z-20 crib, located southeast of the PFP facilities. The Z-20 crib consists of three parallel, perforated, polyvinyl chloride pipes, $460 \mathrm{~m}(1,500 \mathrm{ft}) 1 \mathrm{ong}$. 
Table 5-3. Tanks Considered External to Gloveboxes or Hoods. (7 sheets)

\begin{tabular}{|c|c|c|c|c|c|c|c|c|c|}
\hline \multirow[t]{2}{*}{$\begin{array}{l}\text { Building } \\
\text { number }\end{array}$} & \multirow[t]{2}{*}{$\begin{array}{c}\text { Room } \\
\text { number }\end{array}$} & \multirow[t]{2}{*}{ Tanks } & \multirow[t]{2}{*}{ Tank type } & \multicolumn{2}{|c|}{$\begin{array}{c}\text { Dimensions } \\
(\mathrm{m})\end{array}$} & \multirow{2}{*}{$\begin{array}{c}\text { Number } \\
\text { of } \\
\text { tanks }\end{array}$} & \multirow{2}{*}{$\begin{array}{c}\text { Total } \\
\text { volyme } \\
\left(m^{3}\right)\end{array}$} & \multirow[t]{2}{*}{ Chemical } & \multirow[t]{2}{*}{ Classification } \\
\hline & & & & Diameter & Height & & & & \\
\hline $234-52$ & 166 & Storage $^{\mathrm{a}}$ & -- & 0.15 & 6.10 & 2 & 0.22 & HS & DSH \\
\hline \multirow[t]{5}{*}{$234-52$} & \multirow[t]{5}{*}{337} & $\mathrm{HN}-\mathrm{A}$ & 304-L stainless steel & $\cdots$ & -- & 1 & 0.30 & HS & DSH \\
\hline & & $\mathrm{HN}-\mathrm{B}$ & Fiberglass and reinforced plastic & $\cdots$ & $\cdots$ & 1 & 2.00 & $1.8-2.2 \mathrm{H}, \mathrm{HNO}_{3}$ & DSW \\
\hline & & $\mathrm{HN}-\mathrm{C}$ & Schedule 10 s $304-L$ stainless steel & 0.41 & 2.74 & 1 & 0.36 & $\mathrm{HS}, \mathrm{HN}, \mathrm{HNO}_{3}$ & DSH \\
\hline & & HN-D & Schedule 105 304-L stainless steel & 0.41 & 2.74 & 1 & 0.36 & $\mathrm{HS}, \mathrm{HN}_{1} \mathrm{HNO}_{3}$ & DSH \\
\hline & & $\mathrm{HN}-\mathrm{E}$ & 304-L stainless steel & $-\cdot$ & $\cdots$ & 1 & 0.90 & HN & DSH \\
\hline \multirow[t]{12}{*}{$234-52$} & \multirow[t]{12}{*}{336} & H & Unknown & $-\cdot$ & $\cdots$ & 1 & 0.60 & $\mathrm{HNO}_{3}$, Oxalic acid & DSH \\
\hline & & 1 & Unknown & $\cdots$ & $\cdots$ & 1 & 0.60 & $\mathrm{HNO}_{3}$, Oxalic acid & DSH \\
\hline & & $F-1$ & Unknown & $-\cdot$ & -- & 1 & 0.60 & Oxalic acid & DSH \\
\hline & & $F-2$ & Unknown & $\cdots$ & $-\cdot$ & 1 & 0.60 & Oxalic acid & DSH \\
\hline & & c & Unknown & $\cdots$ & $-\cdot$ & 1 & 0.60 & $\mathrm{HNO}_{3}$ & DSH \\
\hline & & $D-1$ & Unknown & $\because$ & $\cdots$ & 1 & 0.18 & Unknown & $\mathrm{DSH}^{\mathrm{b}}$ \\
\hline & & $0-2$ & Unknown & $\because$ & $\cdots$ & 1 & 0.18 & Unknown & $\mathrm{DSH}^{\mathrm{b}}$ \\
\hline & & G-1 & Unknown & $\cdots$ & $\because$ & 1 & 0.18 & Unknown & $\mathrm{DSH}^{\mathrm{b}}$ \\
\hline & & G-2 & Unknown & $-\cdot$ & $-\cdot$ & 1 & 0.18 & Demineralized water & SH \\
\hline & & E-1 & Unknown & $\cdots$ & $\cdots$ & 1 & 0.18 & Unknown & $\mathrm{DSH}^{\mathrm{b}}$ \\
\hline & & $E-3$ & Unknown & $\cdots$ & $-\cdot$ & 1 & 0.10 & $\mathrm{H}_{2} \mathrm{O}_{2}$ & DSH \\
\hline & & HFI-1 & Unknown & $\because$ & $-\cdot$ & 1 & 0.90 & Unknown & $\mathrm{DSW}^{\mathrm{b}}$ \\
\hline \multirow[t]{8}{*}{$234-52$} & \multirow[t]{8}{*}{337} & D-15 & Unknown & $\cdots$ & $\cdots$ & 1 & 0.90 & $\mathrm{KMnO}_{4}$ & DSH \\
\hline & & D-16 & Stainless steel & 0.7 & 0.86 & 1 & 0.33 & $\mathrm{DBBP}-\mathrm{CCl}_{4}$ & DSH \\
\hline & & $k-3$ & Stainless steel & 0.7 & 0.86 & 1 & 0.33 & $\mathrm{CCl}_{4}$ & DSH \\
\hline & & G-11 & Stainless steel & 1.06 & 1.24 & 1 & 1.09 & $\mathrm{HNO}_{3}, \mathrm{KOH}$ & DSH \\
\hline & & TK-2 & Polyvinyl chloride & 0.61 & 0.91 & 1 & 0.27 & $\mathrm{HNO}_{3}-\mathrm{HF}$ & DSH \\
\hline & & $0-34$ & Stainless steel & 1.06 & 1.24 & 1 & 1.09 & $\mathrm{HaOH}$ & DSH \\
\hline & & $F 2 X$ & Unknown & $\cdots$ & $\ddot{-\cdot}$ & $\because$ & $\because$ & Unknown & $\cdots$ \\
\hline & & $\mathrm{J}-11$ & Unknown & $\cdots$ & $\cdots$ & 1 & 0.10 & $\mathrm{KMnO}_{4}$ & DSH \\
\hline
\end{tabular}


Table 5-3. Tanks Considered External to Gloveboxes or Hoods. (7 sheets)

\begin{tabular}{|c|c|c|c|c|c|c|c|c|c|}
\hline $\begin{array}{l}\text { Building } \\
\text { number }\end{array}$ & $\begin{array}{c}\text { Room } \\
\text { number }\end{array}$ & Tanks & Tank type & \multicolumn{2}{|c|}{$\begin{array}{c}\text { Dimensions } \\
(\mathrm{m})\end{array}$} & $\begin{array}{c}\text { Number } \\
\text { of } \\
\text { tanks }\end{array}$ & $\begin{array}{c}\text { Total } \\
\text { volyme } \\
\left(\mathrm{m}^{\prime}\right)\end{array}$ & Chemical & Classification \\
\hline \multirow[t]{21}{*}{$236-2$} & \multirow[t]{21}{*}{12} & $T K-01$ & 304-L stainless steel & 0.05 & 1.22 & 1 & 0.002 & 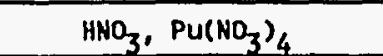 & TRU MH \\
\hline & & TK-03-07 & Unknown & $-\cdot$ & $-\cdot$ & $-\cdot$ & $-\cdot$ & Unknown & 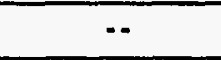 \\
\hline & & $\mathrm{TK}-08$ & Unknown & $\cdots$ & $\cdots$ & 1 & 0.09 & $\mathrm{HNO}_{3}, \mathrm{Pu}\left(\mathrm{NO}_{3}\right)_{4}$ & TRU MH \\
\hline & & TK-8.1 & 304-L stainless steel & 0.06 & 0.30 & 1 & 0.001 & $\mathrm{HNO}_{3}, \mathrm{Pu}\left(\mathrm{NO}_{3}\right)_{4}$ & TRU MH \\
\hline & & $T K-10$ & 304-L stainless steel & 0.15 & 5.18 & 1 & 0.09 & $\mathrm{HNO}_{3}, \mathrm{Pu}\left(\mathrm{NO}_{3}\right)_{4}$ & TRU MH \\
\hline & & $T K-12$ & 304-L stainless steel & 0.15 & 5.18 & 1 & 0.09 & $\mathrm{HNO}_{3}, \mathrm{Pu}\left(\mathrm{NO}_{3}\right)_{4}$ & TRU MH \\
\hline & & TK-14 & Unknown & $\cdots$ & $\cdots$ & -- & -- & Unknown & -- \\
\hline & & $T K-15$ & $304-L$ stainless steel & 0.13 & 1.14 & 1 & 0.02 & $\mathrm{Pu}\left(\mathrm{NO}_{3}\right)_{4}$, ANN & TRU MH \\
\hline & & TK-16 & 304-L stainless steel & 0.13 & 4.67 & 1 & 0.06 & $\mathrm{Pu}\left(\mathrm{NO}_{3}\right)_{4}, \mathrm{Pu}^{+4}-2 \mathrm{TBP}-4 \mathrm{NO}_{3}$ & TRU MH \\
\hline & & TK-17 & 304-L stainless steel & 0.13 & 4.67 & 4 & 0.25 & $\mathrm{Pu}\left(\mathrm{NO}_{3}\right)_{4}$ & TRU MH \\
\hline & & TK-21 & $304-L$ stainless steel & 0.15 & 1.83 & 1 & 0.03 & $\mathrm{Pu}\left(\mathrm{NO}_{3}\right)_{4}$ & TRU MH \\
\hline & & TK-22 & 304-L stainless steel & 0.15 & 1.12 & 1 & 0.02 & $\mathrm{Pu}\left(\mathrm{NO}_{3}\right)_{4}$ & TRU MH \\
\hline & & $\mathrm{TK}-23$ & 304-L stainless steel & 0.13 & 1.12 & 1 & 0.01 & $\mathrm{Pu}\left(\mathrm{NO}_{3}\right)_{4}$ & TRU MH \\
\hline & & $\mathrm{TK}-23.1$ & 304-L stainless steel & 0.08 & 0.33 & 1 & 0.002 & $\mathrm{Pu}\left(\mathrm{NO}_{3}\right)_{4}$ & TRU MH \\
\hline & & $T K-24$ & 304-L stainless steel & 0.13 & 1.12 & 1 & 0.01 & $\left.\mathrm{Pu} \mathrm{NO}_{3}\right)_{4}$ & TRU HH \\
\hline & & $\mathrm{TK}-25$ & $304-L$ stainless steel & 0.13 & 1.12 & 4 & 0.06 & $\mathrm{Pu}\left(\mathrm{NO}_{3}\right)_{4}$ & TRU MU \\
\hline & & $\mathrm{TK}-25.1$ & $304-L$ stainless steel & 0.08 & 0.33 & 1 & 0.002 & $\mathrm{Pu}\left(\mathrm{NO}_{3}\right)_{4}$ & TRU MH \\
\hline & & $T K-26$ & 304-L stainless steel & 0.13 & 1.12 & 4 & 0.06 & $\mathrm{Pu}\left(\mathrm{NO}_{3}\right)_{4}$ & TRU MH \\
\hline & & TK-26.1 & 304-L stainless steel & 0.08 & 0.33 & 1 & 0.002 & $\mathrm{Pu}\left(\mathrm{NO}_{3}\right)_{4}$ & TRU MH \\
\hline & & $T K-27$ & $304-L$ stainless steel & 0.13 & 1.12 & 4 & 0.06 & $\left.\mathrm{Pu} \mathrm{NO}_{3}\right)_{4}$ & TRU MH \\
\hline & & $T K-28$ & 304-L stainless steel & 0.13 & 1.14 & 4 & 0.06 & $\mathrm{Pu}\left(\mathrm{HO}_{3}\right)_{4}$ & TRU MH \\
\hline
\end{tabular}


Table 5-3. Tanks Considered External to Gloveboxes or Hoods. (7 sheets)

\begin{tabular}{|c|c|c|c|c|c|c|c|c|c|}
\hline \multirow[t]{2}{*}{$\begin{array}{l}\text { Building } \\
\text { number }\end{array}$} & \multirow[t]{2}{*}{$\begin{array}{c}\text { Room } \\
\text { number }\end{array}$} & \multirow[t]{2}{*}{ Tanks } & \multirow[t]{2}{*}{ Tank type } & \multicolumn{2}{|c|}{$\begin{array}{c}\text { Dimensions } \\
(\mathrm{m})\end{array}$} & \multirow{2}{*}{$\begin{array}{l}\text { Number } \\
\text { of } \\
\text { tanks }\end{array}$} & \multirow{2}{*}{$\begin{array}{l}\text { Total } \\
\text { volume } \\
\left(\mathrm{m}^{3}\right)\end{array}$} & \multirow[t]{2}{*}{ Chemical } & \multirow{2}{*}{ Classification } \\
\hline & & & & Diameter & Height & & & & \\
\hline \multirow{27}{*}{$\begin{array}{l}236-2 \\
\text { (cont.) }\end{array}$} & \multirow{27}{*}{$\begin{array}{l}12 \\
\text { (cont.) }\end{array}$} & $\mathrm{TK}-30$ & 304-L stainless steel & 0.15 & 1.07 & 4 & 0.08 & $\mathrm{HNO}_{3}, \mathrm{Pu}\left(\mathrm{NO}_{3}\right)_{4}$ & TRU MH \\
\hline & & $T K-31$ & 304-L stainless steel & 0.15 & 3.84 & 1 & 0.07 & $\left.\mathrm{Pu} \mathrm{NO}_{3}\right)_{4}$ & TRU MW \\
\hline & & $T K-32$ & 304-L stainless steel with Pyrex & 0.10 & 15.24 & 1 & 0.12 & $\mathrm{Pu}\left(\mathrm{NO}_{3}\right)_{4}, \mathrm{Pu}^{+4}-2 \mathrm{TBP}-4 \mathrm{NO}_{3}$ & TRU MH \\
\hline & & $T K-32 A$ & Glass & 0.15 & 1.07 & 1 & 0.02 & Am, NaOH, ANM & TRU MH \\
\hline & & $\mathrm{TK}-33$ & 304-L stainless steel with Pyrex & 0.10 & 15.24 & 1 & 0.12 & $\mathrm{Pu}\left(\mathrm{NO}_{3}\right)_{4}, \mathrm{Pu}^{+4}-2 \mathrm{TBP}-4 \mathrm{NO}_{3}$ & TRU MH \\
\hline & & $T K-34$ & Teflon ${ }^{2}-$ lined stainless steel & 0.15 & 6.05 & 1 & 0.11 & $\mathrm{Pu}\left(\mathrm{NO}_{3}\right)_{4}, \mathrm{Pu}^{+4}-2 \mathrm{TBP}-4 \mathrm{NO}_{3}$ & TRU MH \\
\hline & & $\mathrm{TK}-34 \mathrm{~A}$ & Glass & 0.15 & 0.30 & 1 & 0.005 & $\mathrm{Pu}\left(\mathrm{NO}_{3}\right)_{4}, \mathrm{Pu}^{+4}-2 \mathrm{TBP}-4 \mathrm{NO}_{3}$ & TRU MH \\
\hline & & TK-34.1 & 304-L stainless steel & 0.06 & 0.30 & 1 & 0.001 & $\mathrm{Pu}\left(\mathrm{NO}_{3}\right)_{4}, \mathrm{Pu}^{+4}-2 \mathrm{TBP}-4 \mathrm{NO}_{3}$ & TRU MW \\
\hline & & TK-35 & Unknown & $-\cdot$ & $\cdots$ & $-\cdot$ & $\cdots$ & Unknown & -- \\
\hline & & $T K-36$ & 304-L stainless steel & 0.13 & 1.14 & 4 & 0.06 & $\mathrm{Pu}\left(\mathrm{NO}_{3}\right)_{4}$ & TRU MH \\
\hline & & TK-37 & 304-L stainless steel & 0.13 & 4.17 & 4 & 0.22 & $\mathrm{TBP}, \mathrm{CCl}_{4}$ & DSH \\
\hline & & TK-37.1 & 304-L stainless steel & 0.08 & 0.33 & 1 & 0.002 & $\mathrm{TBP}, \mathrm{CCl}_{4}$ & DSH \\
\hline & & TK-38 & 304-L stainless steel & 0.13 & 4.17 & 4 & 0.22 & $\mathrm{TBP}, \mathrm{CCl}_{4}$ & DSH \\
\hline & & TK-38.1 & 304-L stainless steel & 0.08 & 0.33 & 1 & 0.002 & $\mathrm{TBP}_{1} \mathrm{CCl}_{4}$ & DSH \\
\hline & & TK-39 & 304-L stainless steel & 0.13 & 4.17 & 4 & 0.22 & $\mathrm{Pu}\left(\mathrm{NO}_{3}\right)_{4}$ & TRU MH \\
\hline & & TK-39.1 & 304-L stainless steel & 0.10 & 0.30 & 1 & 0.002 & $\mathrm{Pu}\left(\mathrm{NO}_{3}\right)_{4}$ & TRU MH \\
\hline & & TK-39.2 & 304-L stainless steel & 0.10 & 0.30 & 1 & 0.002 & $\mathrm{Pu}\left(\mathrm{NO}_{3}\right)_{4}$ & TRU MH \\
\hline & & $T K-40$ & 304-L stainless steel & 0.13 & 4.17 & 4 & 0.22 & $\mathrm{Pu}\left(\mathrm{NO}_{3}\right)_{4}$ & TRU MH \\
\hline & & $T K-41$ & 304-L stainless steel & 0.13 & 1.14 & 4 & 0.06 & $\mathrm{Pu}\left(\mathrm{NO}_{3}\right)_{4}$ & TRU MH \\
\hline & & $T K-41.1$ & 304-L stainless steel & 0.10 & 0.30 & 1 & 0.002 & $\mathrm{Pu}\left(\mathrm{NO}_{3}\right)_{4}$ & TRU MH \\
\hline & & $T K-41.2$ & 304-L stainless steel & 0.10 & 0.30 & 1 & 0.002 & $\mathrm{Pu}\left(\mathrm{NO}_{3}\right)_{4}$ & TRU MH \\
\hline & & $T K-42$ & 304-L stainless steel & 0.13 & 1.14 & 4 & 0.06 & $\mathrm{Pu}\left(\mathrm{NO}_{3}\right)_{4}$ & TRU MH \\
\hline & & $T K-43$ & Titanium & 0.10 & 3.28 & 1 & 0.03 & $\mathrm{Pu}\left(\mathrm{NO}_{3}\right)_{4}$ & TRU MH \\
\hline & & TK-44 & 304-L stainless steel & 0.15 & 1.12 & 1 & 0.02 & $\mathrm{Pu}\left(\mathrm{HO}_{3}\right)_{4}, \mathrm{Pu}^{+4}-2 \mathrm{TBP}-4 \mathrm{NO}_{3}$ & TRU MH \\
\hline & & $T K-45$ & 304-L stainless steel & 0.13 & 1.14 & 4 & 0.06 & $\mathrm{Pu}\left(\mathrm{NO}_{3}\right)_{4}$ & TRU MH \\
\hline & & $T K-46$ & 304-L stainless steel & 0.13 & 1.14 & 4 & 0.06 & $\mathrm{Pu}\left(\mathrm{NO}_{3}\right)_{4}$ & TRU MH \\
\hline & & TK-49 & 304-L stainless steel & 0.13 & 1.14 & 4 & 0.06 & $\mathrm{Pu}\left(\mathrm{HO}_{3}\right)_{4}$ & TRU MH \\
\hline
\end{tabular}




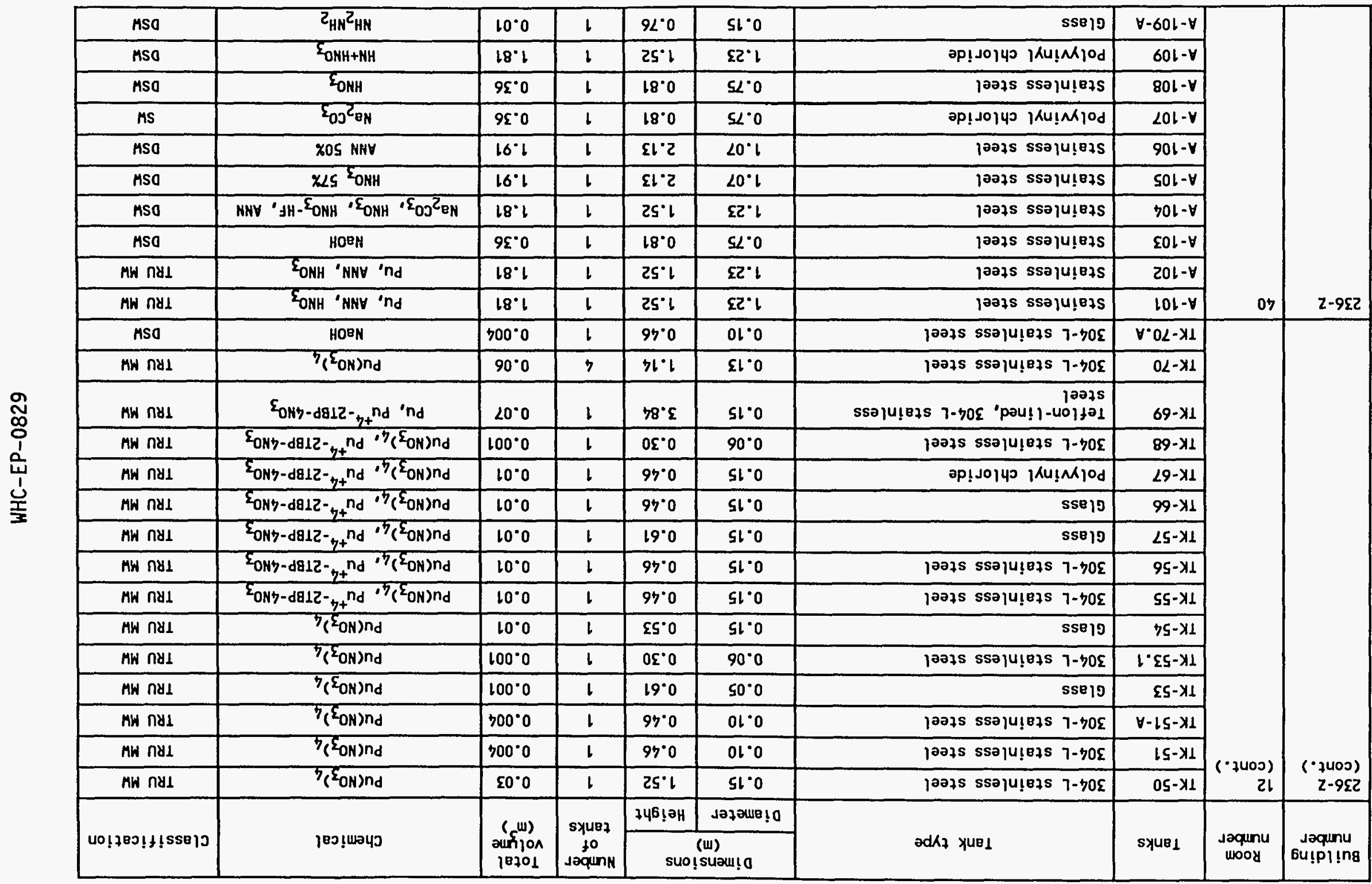


Table 5-3. Tanks Considered External to Gloveboxes or Hoods. (7 sheets)

\begin{tabular}{|c|c|c|c|c|c|c|c|c|c|}
\hline \multirow[t]{2}{*}{$\begin{array}{l}\text { Building } \\
\text { number }\end{array}$} & \multirow[t]{2}{*}{$\begin{array}{c}\text { Room } \\
\text { number }\end{array}$} & \multirow[t]{2}{*}{ Tanks } & \multirow[t]{2}{*}{ Tank type } & \multicolumn{2}{|c|}{$\begin{array}{l}\text { Dimensions } \\
\text { (m) }\end{array}$} & \multirow{2}{*}{$\begin{array}{c}\text { Number } \\
\text { of } \\
\text { tanks }\end{array}$} & \multirow{2}{*}{$\begin{array}{c}\text { Total } \\
\text { volyme } \\
\left(m^{\prime}\right)\end{array}$} & \multirow[t]{2}{*}{ Chemical } & \multirow[t]{2}{*}{ Classification } \\
\hline & & & & Diameter & Height & & & & \\
\hline \multirow{23}{*}{$\begin{array}{l}236-2 \\
\text { (cont.) }\end{array}$} & \multirow{23}{*}{$\begin{array}{l}40 \\
\text { (cont.) }\end{array}$} & $A-110$ & Polyvinyl chloride & 0.75 & 0.75 & 1 & 0.33 & $\mathrm{HNO}_{3}, \mathrm{HF}, \mathrm{ANH}$ & DSH \\
\hline & & $A-111$ & Stainless steel & 0.75 & 0.81 & 1 & 0.36 & Mistron, $\mathrm{NaNO}_{2}$ & DSH \\
\hline & & $A-112$ & Stainless steel & 0.75 & 0.81 & 1 & 0.36 & Mistron, $\mathrm{HNO}_{3}, \mathrm{ANH}$ & DSH \\
\hline & & $A-113$ & Stainless steel & 0.75 & 0.81 & 1 & 0.36 & $\mathrm{HNO}_{3}-\mathrm{NaNO}_{3}$ & DSH \\
\hline & & A-114 & Stainless steel & 0.75 & 0.81 & 1 & 0.36 & TBP & DSH \\
\hline & & A-115 & Stainless steel & 0.75 & 0.81 & 1 & 0.36 & $\mathrm{CCl}_{4}$ & DSW \\
\hline & & A-116 & Stainless steel & 0.75 & 0.81 & 1 & 0.36 & Antifoam, $\mathrm{HNO}_{3}$ & DSW \\
\hline & & $A-117$ & Stainless steel & 0.75 & 0.91 & 1 & 0.40 & B-acid & DSH \\
\hline & & $A-118$ & Stainless steel & 0.20 & 0.63 & 1 & 0.02 & $\mathrm{NaNO}_{2}$ & DSH \\
\hline & & $A=119$ & staintess steel & 0.46 & 0.61 & 1 & 0.10 & $\mathrm{HNO}_{3} \mathrm{T2}$ & DSH \\
\hline & & TK-120 & 304-L stainless steel & 1.35 & 2.26 & 1 & 3.23 & $\mathrm{Pu}^{+4}-2 \mathrm{TBP}^{-4 \mathrm{NO}_{3}}$ & TRU MH \\
\hline & & TK-121 & 304-L stainless steel & 1.35 & 2.26 & 1 & 3.23 & $\mathrm{Pu}, \mathrm{U}, \mathrm{HNO}_{3}, \mathrm{HN}$ & TRU MH \\
\hline & & $T K-122$ & $\begin{array}{l}304-L \text { stainless steel Intalox } \\
\text { saddles }\end{array}$ & 0.10 & 7.01 & 1 & 0.06 & $\mathrm{Pu}, \mathrm{U}, \mathrm{HNO}_{3}, \mathrm{HN}$ & TRU MH \\
\hline & & $T K-122-A$ & Carbon steel & 0.15 & 3.66 & 1 & 0.06 & Air surge tank & TRU $M H^{b}$ \\
\hline & & TK-123 & $\begin{array}{l}304-L \text { stainless steel Intalox } \\
\text { saddles }\end{array}$ & 0.10 & 7.01 & 1 & 0.06 & $\mathrm{Pu}\left(\mathrm{NO}_{3}\right)_{4}$ & TRU MH \\
\hline & & $T K-123-A$ & Carbon steel & 0.15 & 3.66 & 1 & 0.06 & Air surge tank & TRU $M H^{b}$ \\
\hline & & TK-124 & Kynar $^{4}-l$ ined stainless steel & 0.10 & 5.18 & 2 & 0.08 & $\mathrm{HNO}_{3}$ & DSW \\
\hline & & TK-124.1 & 304-L stainless steel & 0.06 & 0.30 & 1 & 0.001 & $\mathrm{HNO}_{3}$ & DSH \\
\hline & & TK-125 & 304-L stainless steel & 0.15 & 0.81 & 1 & 0.01 & Condenser surge tank & TRU $M H^{b}$ \\
\hline & & TK-126 & 304-L stainless steel & 0.13 & 4.67 & 4 & 0.25 & $\mathrm{Pu}\left(\mathrm{NO}_{3}\right)_{4}, \mathrm{Pu}^{+4}-2 \mathrm{TBP}-4 \mathrm{NO}_{3}$ & TRU MH \\
\hline & & TK-127 & 304-L stainless steel & 0.13 & 4.67 & 4 & 0.25 & $\mathrm{Pu}\left(\mathrm{NO}_{3}\right)_{4}$ & TRU MH \\
\hline & & TK-128 & 304-L stainless steel & 0.13 & 1.14 & 4 & 0.06 & $\mathrm{Pu}\left(\mathrm{HO}_{3}\right)_{4}$ & TRU MH \\
\hline & & TK-WM-1 & 304-L stainless steel & 0.16 & 4.37 & 3 & 0.26 & $\mathrm{HNO}_{3}, \mathrm{Pu}\left(\mathrm{NO}_{3}\right)_{4}, \mathrm{HF}$ & TRU MH \\
\hline \multirow[t]{3}{*}{$236-2$} & \multirow[t]{3}{*}{ Canyon } & TK-WE-1 & 304-L stainless steel & 0.16 & 7.92 & 1 & 0.16 & $\mathrm{Pu}, \mathrm{Am}, \mathrm{DBBP}-\mathrm{CCl}_{4}$ & TRU MH \\
\hline & & TK-WS-1 & Kynar-lined stainless steel & 0.10 & 6.56 & 1 & 0.05 & $\mathrm{Pu}, \mathrm{Am}, \mathrm{DBBP}-\mathrm{CCl} 4, \mathrm{HHO}_{3}$ & TRU MH \\
\hline & & TK-WS-2 & Kynar-lined staintess steel & 0.10 & 4.88 & 1 & 0.04 & $\mathrm{Pu}, \mathrm{HHO}_{3}, \mathrm{HF}$ & TRU NH \\
\hline
\end{tabular}


Table 5-3. Tanks Considered External to Gloveboxes or Hoods. (7 sheets)

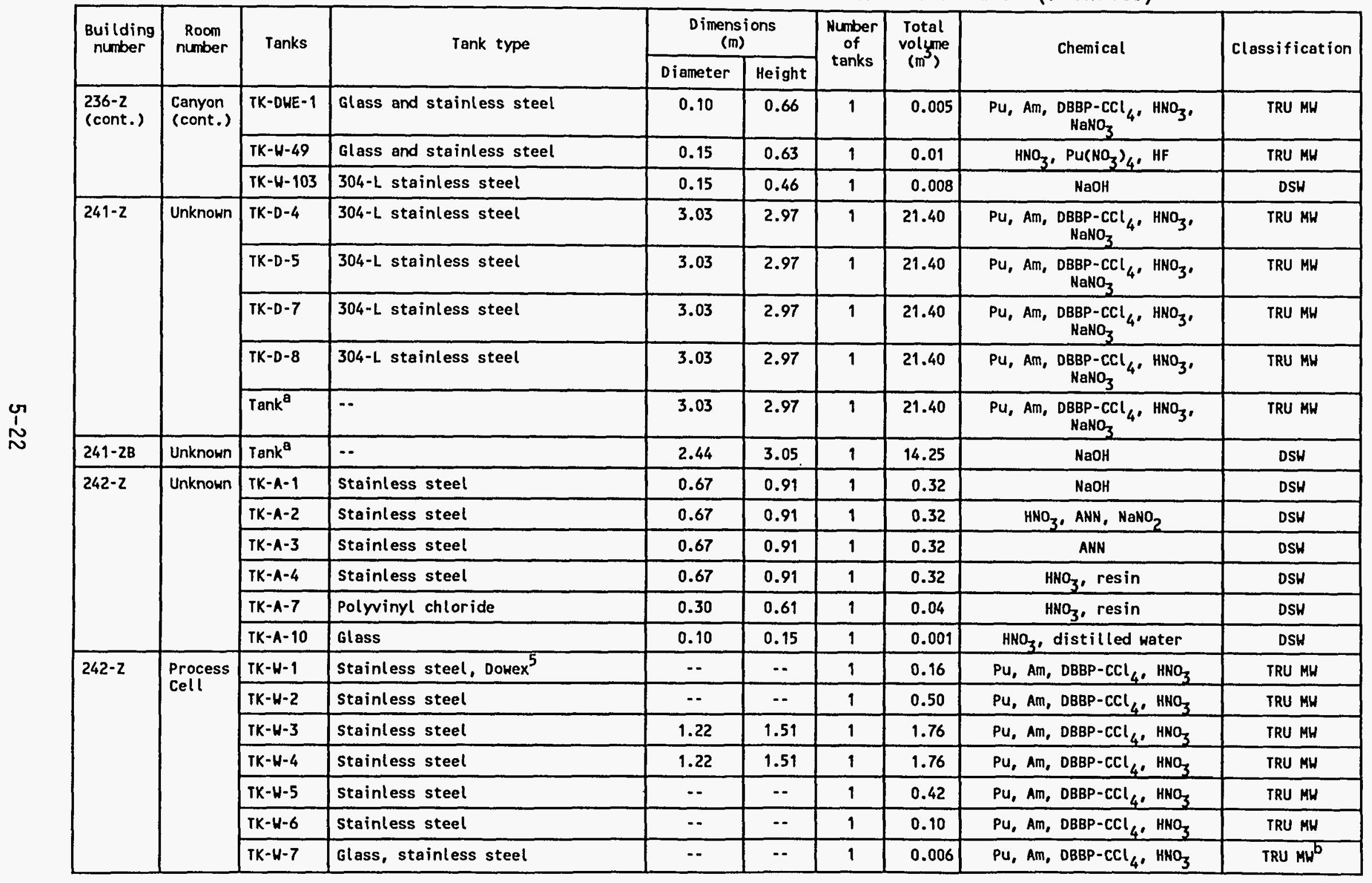


Table 5-3. Tanks Considered External to Gloveboxes or Hoods. (7 sheets)

\begin{tabular}{|c|c|c|c|c|c|c|c|c|c|}
\hline \multirow[t]{2}{*}{$\begin{array}{l}\text { Building } \\
\text { number }\end{array}$} & \multirow[t]{2}{*}{$\begin{array}{c}\text { Room } \\
\text { number }\end{array}$} & \multirow[t]{2}{*}{ Tanks } & \multirow[t]{2}{*}{ Tank type } & \multicolumn{2}{|c|}{$\begin{array}{c}\text { Dimensions } \\
(\mathrm{m})\end{array}$} & \multirow[t]{2}{*}{$\begin{array}{l}\text { Number } \\
\text { of } \\
\text { tanks }\end{array}$} & \multirow[t]{2}{*}{$\begin{array}{l}\text { Total } \\
\text { volưme } \\
\left(m^{3}\right)\end{array}$} & \multirow[t]{2}{*}{ Chemical } & \multirow[t]{2}{*}{ Classification } \\
\hline & & & & Diameter & Height & & & & \\
\hline \multirow{6}{*}{$\begin{array}{l}242-2 \\
\text { (cont.) }\end{array}$} & \multirow{6}{*}{$\begin{array}{l}\text { Process } \\
\text { Cell } \\
\text { (cont.) }\end{array}$} & TK-W-8-3 & Stainless steel & 0.09 & 0.60 & 1 & 0.004 & $\mathrm{Pu}, \mathrm{Am}, \mathrm{DBBP}-\mathrm{CCl}_{4}, \mathrm{HHO}_{3}$ & TRU MH \\
\hline & & IK-U-8-4 & Stainless steel & 0.09 & 0.60 & 1 & 0.004 & $\mathrm{Pu}, \mathrm{Am}, \mathrm{DBBP}-\mathrm{CCl}_{4}, \mathrm{HNO}_{3}$ & TRU MH \\
\hline & & $T K-H-10$ & Glass, stainless steel & $\cdots$ & $\cdots$ & 1 & 0.009 & $\mathrm{Pu}, \mathrm{Am}, \mathrm{DBBP}-\mathrm{CCl}_{4}, \mathrm{HNO}_{3}$ & TRU MH \\
\hline & & $T K-4-11$ & Stainless steel & 0.09 & 0.60 & 1 & 0.004 & $\mathrm{Pu}, \mathrm{Am}, \mathrm{DBBP}-\mathrm{CCl}_{4}, \mathrm{HNO}_{3}$ & TRU MW \\
\hline & & $T K-H-12$ & Stainless steel & 1.82 & 1.51 & 1 & 3.93 & $\mathrm{Pu}, \mathrm{Am}, \mathrm{HF}, \mathrm{HNO}_{3}$ & TRU MH \\
\hline & & $T K-W-13$ & Stainless steel & 1.51 & 1.51 & 1 & 2.70 & $\mathrm{Pu}, \mathrm{Am}, \mathrm{HF}, \mathrm{HNO}_{3}$ & TRU MH \\
\hline & & & & \multicolumn{3}{|c|}{$\begin{array}{l}\text { Total volume: } \\
\text { DSH volume: } \\
\text { TRU MH volume: }\end{array}$} & $\begin{array}{c}173.20^{c} \\
40.26 \\
132.94\end{array}$ & & \\
\hline
\end{tabular}

b Halkdown est imate.

Estimate based on process information.

1 Pyrex is a trademark of Corning Glass Horks.

Teflon is a trademark of E. I. Du Pont de Nemours \& Company.

3 Intalox is a trademark of Norton Chemical Process Products.

5 Kynar is a trademark of Pennwalt Corporation.

Dowex is a trademark of Dow Chemical Company.

Am = Americium.

ANN $=$ Aluminum nitrate nanohydrate.

B acid $=12 \mathrm{M} \mathrm{HNO}_{3}+0.35 \mathrm{MHF}$.

$\mathrm{CCl}_{4}=$ Carbon tetrach toride.

DBBP = Dibutylbiphenyl.

DSH $=$ Dangerous solid waste

$\mathrm{HF}=$ Hydrofluoric acid.

$H N=$ Hydroxylamine nitrate.

$\mathrm{HNO}_{3}$ a Hitric acid.

$\mathrm{H}_{2} \mathrm{O}_{3}=$ Hydrogen peroxide

${ }_{H S}=$ Hydroxylamine sulfate.

$\mathrm{KMnO}=$ Potassium permanganate

KOH = Potassium hydroxide.

$\mathrm{Na}_{2} \mathrm{CO}_{3}=$ Sodium carbonate.

NanO $=$ Sodium nitrate.

$\mathrm{NaOH}=$ Sodium hydroxide.

$\mathrm{Pu}=$ Plutonium.

$\mathrm{Pu}\left(\mathrm{NO}_{3}\right)_{1}=$ Plutonium nitrate

$S H=$ Sanitary waste.

TBP = Tributyl phosphate.

TRU MH = Transuranic mixed Haste.

$U=$ Uranium. 
The central pipe is $25.4 \mathrm{~cm}$ (10 in.) in diameter with a $15.2-\mathrm{cm}-(6-$ in.-) diameter pipe located to each side. After five to six years of operation, percolation rates in the $Z-20$ crib had decreased to the point that $Z-20 \mathrm{crib}$ stream flow exceeded $Z-20$ crib disposal capacity on occasion. Increased disposal capacity was provided by the installation of 11 drain wells.

To reduce the volume of waste water being discharged into the $Z-20 \mathrm{crib}$, the B-680 and C-040 Projects were approved. These projects segregated the process-equipment cooling water by installing a closed loop cooling system (CLCS), consisting of a primary closed cooling (PCW) loop and a secondary closed cooling (SCH) loop. The flow of process cooling water to the Z-20 crib was halted in January 1994 when the CLCS began operating. The PCW and SCW include eight intermediate flat-plate heat exchangers, redundant recirculation pumps, and two evaporative-type fluid coolers. The SCH acts as a heat sink to the PCW through the intermediate heat exchangers. The PCW, which cools the process equipment, operates at a lower pressure than the SCW to ensure that a breach between the systems would cause a flow towards the PCW (and, hence, the process equipment) (Navarro 1994).

The B-680 Project provided a CLCS for equipment and processes in Buildings $236-Z$ and $234-5 Z$ that represented a potential for contaminating once-through cooling water. The B-680 CLCS installed two PCW 1oops and an SCW loop. One PCW loop provides c00ling to the east and west gallery gloveboxes and gloveboxes 5 and 6 in room 4l. The second PCW loop provides sealing/cooling water to two air sample vacuum pumps located in room 35 . Provisions for a third 10op have been included to service equipment (yet to be identified) in Building 234-5Z (Navarro 1994).

The C-040 Project consisted of four PCW loops for cooling and an extension of the SCW 1oop for transferring heat from these primary loops located in Building 291-Z. The PCW provides sealing/cooling water to two 43-cm Hg (17-in. Hg) air sample vacuum pumps and two process air compressors in room 501, Building 291-Z. The process air compressors were installed under Project $\mathrm{C}-171$. The $\mathrm{C}-040 \mathrm{SCW}$ 100p is an extension of and ties to the B-680 SCW loop at valves V-CLC-9 and V-CLC-10 (Navarro 1994).

The Z-20 crib is the only crib currently used (active) by the PFP. However, many cribs, ditches, and trenches have been utilized by the PFP in the past. Table 5-4 is a list of these and their service (from $\mathrm{H}-2-32528$ ).

The amount of solid waste expected to result from the D\&D of the crib system is difficult to predict because it is not known at this point whether the cribs will be remediated in place or processed elsewhere. For this reason, the cribs were considered outside the scope of this project. Drain lines to the cribs, ditches, and trenches were included.

Building 241-Z covers a below grade, 1iquid-waste collection system containing five tanks in five separate covered cells. This Radioactive Liquid Waste Facility is used for intermediate storage and neutralization of PFP aqueous wastes and is still active. After neutralization, wastes are pumped to the 244-TX Tank Farm. A steam jet also is provided for transfer of the pump heel. Ventilation is provided for the cells and the tanks but not for 
Table 5-4. Cribs, Ditches, and Trenches Used by PFP in the Past.

\begin{tabular}{|c|c|c|}
\hline Number & Description & Service \\
\hline $\begin{array}{c}2-1 \\
\text { (ditch) }\end{array}$ & Ditch (backfilled) & $\begin{array}{l}\text { Cooling water, steam } \\
\text { condensate }\end{array}$ \\
\hline $\begin{array}{c}\mathrm{z}-1 \\
(\mathrm{crib})\end{array}$ & $\begin{array}{l}3.7 \times 3.7 \times 4.3 \mathrm{~m}(12 \times 12 \times 14 \mathrm{ft}) \\
\text { timbered cavern }\end{array}$ & D-6 wastes \\
\hline$Z-1 A$ & 20.3-cm (8-in.) clay pipe tile field & $\begin{array}{l}\text { Reclamation salt waste (pipe } \\
\text { trench) specific retention }\end{array}$ \\
\hline $\mathrm{Z}-2$ & $\begin{array}{l}3.7 \times 3.7 \times 4.3 \mathrm{~m}(12 \times 12 \times 14 \mathrm{ft}) \\
\text { timbered cavern }\end{array}$ & D-6 wastes \\
\hline$z-3$ & $\begin{array}{l}\text { 1.2-m- (4-ft-) diameter perforated } \\
\text { culvert pipe, } 20.1 \mathrm{~m}(66 \mathrm{ft}) \text { long }\end{array}$ & D-6 wastes \\
\hline Z-4 & Unknown & 231-Z lab wastes \\
\hline Z-5 & Timbered cribs (two) & $231-Z$ process wastes \\
\hline$Z-6$ & Temporary trench & $231-Z$ process wastes \\
\hline Z-7 & Pipe trench & $231-\mathrm{Z} 1 \mathrm{ab}$ wastes \\
\hline$Z-8$ & $\begin{array}{l}56,781-\mathrm{L}(15,000-\mathrm{ga} 7) \text { tank and } \\
\text { French drain }\end{array}$ & $\begin{array}{l}\text { Silica waste form recuplex } \\
\text { dissolvers }\end{array}$ \\
\hline Z-9 & $\begin{array}{l}9.1 \times 18.3 \text { (at the bottom) } \times 6.1 \mathrm{~m} \\
\text { deep (30 ft } \times 60 \mathrm{ft} \text { (at the bottom) } \\
\times 20 \mathrm{ft} \text { deep trench with a } 0.3-\mathrm{m} \\
(12-i n .) \text { concrete top }\end{array}$ & Recuplex salt waste \\
\hline Z-10 & Reverse we11 & $231-Z$ wastes \\
\hline Z-11 & Open ditch to swamp (backfilled) & $\begin{array}{l}\text { Cooling water, steam } \\
\text { condensate, vacuum pump } \\
\text { sealing water }\end{array}$ \\
\hline Z-12 & $\begin{array}{l}0.3-\mathrm{m}(12-i n .) \text { perforated clay pipe } \\
\text { in gravel } 9.1 \mathrm{~m} \text { deep }(30 \mathrm{ft}) \times 183 \mathrm{~m} \\
\text { long }(600 \mathrm{ft})\end{array}$ & D-6 wastes \\
\hline Z-13 & French drain & 234-5 tunnel drain \\
\hline$Z-14$ & French drain & Evaporator condensate water \\
\hline Z-15 & French drain & Evaporator condensate water \\
\hline Z-16 & Pipe trench & 231-Z lab wastes \\
\hline Z-17 & Temporary trench & Unknown \\
\hline$Z-18$ & $\begin{array}{l}\text { Five } 61-\mathrm{m}(200-\mathrm{ft}) \text { long pipe } \\
\text { trenches with two } 10.2-\mathrm{cm}(4-\mathrm{in}) \text { ) } \\
\text { perforated clay pipes in each trench }\end{array}$ & $\begin{array}{l}\text { Reclamation salt waste (pipe } \\
\text { trench) specific retention }\end{array}$ \\
\hline Z-19 & Open ditch to swamp & $\begin{array}{l}\text { Cooling water, steam } \\
\text { condensate, vacuum pump } \\
\text { sealing water }\end{array}$ \\
\hline
\end{tabular}


the building. The tanks receive aqueous wastes from processes and 7 aboratories in the PFP complex. Such wastes include steam condensates from the 236-Z plutonium product and filtrate concentrators, filtrate evaporator overheads, and 234-5Z vacuum pump seal water. Wastes are batch transferred to tanks for sampling and analysis to determine their composition. Various pipe sizes and compositions are used throughout this system.

The 241-Z-361 settling tank was placed in service in 1949 and remained in continuous service until May 15, 1973. The tank is an underground, steellined, reinforced-concrete structure with a sloping bottom. Its approximate inside dimensions are $4.0 \mathrm{~m}$ by $8.0 \mathrm{~m}$ by $5.5 \mathrm{~m}$ (depth) $(13 \mathrm{ft} \times 26 \mathrm{ft} \times 18 \mathrm{ft}$ ). The tank was filled by jetting from the sump tanks (known as D-4, D-5, D-7, and $D-8)$. Baffles over the inlet and overflow pipes reduced turbulent flow in the tank, permitting particulates in the neutralized waste stream to settle out before the liquid was discharged to the receiving crib. Overflow from the tank flowed to the cribs in Table 5-5, during the given time periods, by gravity feed (Kasper 1981).

Table 5-5. Cribs Fed by Overflow from the 241-Z-361 Settling Tank.

\begin{tabular}{|c|l|}
\hline Crib & \multicolumn{1}{|c|}{ Period of use } \\
\hline Z-1 and Z-2 & June 1949 to June 1952 \\
\hline Z-3 & JuTy 1952 to March 11, 1959 \\
\hline Z-12 & March 12, 1959, to May 15, 1973 \\
\hline
\end{tabular}

The 241-Z-361 settling tank received neutralized, low-salt, aqueous waste streams from the PFP. The sources of these waste streams were

- The hydro-fluorinator offgas water jet (button line)

- Certain cooling waters and condensates

- Lab wastes and hood floor drains.

In addition, from 1961 to 1973, the sump tanks received expended scrubber and wash solutions from the offgas scrubber of the PFP incinerator, which contained dry fly ash (Harlow 1975). On May 15, 1973, use of the 241-Z-361 settling tank was discontinued and all 7 ines to it were blanked (Harlow 1975). The tank was left full of supernate and sludge; the sludge is reportedly $2.4 \mathrm{~m}$ deep (Burns 1978). The plutonium accounted for out of sump tanks $D-4, D-5, D-7$, and $D-8$ to the 241-Z-361 settling tank and the cribs is shown in Table 5-6 (Harlow 1975).

Chemical makeup areas are located in Building 234-52, rooms 336 and 337 , and Building 236-Z, room 40 . Chemical makeup involves mixing chemicals with other chemicals and/or water to provide solutions or organic mixtures necessary to run various processes supporting plutonium operation at the PFP. After the chemicals are prepared, they are either stored or transferred via chemical addition pipe lines to the facilities in which they will be used. 
Table 5-6. Plutonium from Sump Tanks to 241-Z-361 Settling Tank and Cribs.

\begin{tabular}{|c|c|}
\hline Crib & Plutonium (g) \\
\hline$Z-1$ and Z-2 & 199 \\
\hline$Z-3$ & 5,698 \\
\hline$Z-12$ & 25,300 \\
\hline Total to 241-Z-361 & 31,200 \\
\hline
\end{tabular}

Blowers supply dry air to various process gloveboxes to maintain low moisture content in plutonium products, which corrode in moist air or are hygroscopic. Two dry-air generating systems, one electric, one steam, are designed to operate separately, so one unit is operating while the other is on standby. Room air is passed through a roughing filter and refrigerated to remove most of the moisture. It is then passed through a silica gel drying tower and refrigerated again, then through a second silica gel drying tower, and finally passed out to the dry-air header that services the gloveboxes in Building 234-5Z. Flow is regulated by control valves at each glovebox serviced by the header.

Dry air to the repackaging glovebox in room $636,2736-Z B$, is supplied by two air compressors, driven by electric motors, and a drying tower.

Instrument air is distributed throughout the 234-5Z Building for general use in controlling instruments for the ventilation systems, the process systems, and the dry-air systems.

The $66-\mathrm{cm} \mathrm{Hg}$ (26-in. $\mathrm{Hg}$ ) process vacuum system provides high-capacity vacuum service to the PFP facilities for vacuum transfers of 1 iquids and other high-vacuum requirements. It is a contaminated system with a Safety Class 2 designation. A 10.2-cm- (4-in.-) diameter stainless steel pipe header is routed throughout the duct level of the 234-5Z Building with branches serving process areas and the 242-Z and 236-Z Buildings.

The air pulled into this system, in many cases, comes from contaminated enclosures and is usually moist from use as the source of a vacuum transfer of contaminated 1iquid. Thus, each major branch is equipped with demisters. Liquid effluents from the seal-water loop, which may contain trace amounts of contamination, are routed to the 241-Z Building waste tanks for disposal.

Vacuum is provided by two vacuum pumps. The inlet stream to the pumps contains two sets of two-stage HEPA filters. The vacuum pumps discharge water to a trap-silencer tank to separate the seal water from the discharged gas. The gas is routed out of the water trap-silencer tank up through a demister and into the E-4 ventilation system ahead of the final E-4 filter bank (see Section 4.0). A seal-water pump recirculates the seal water from the bottom of the water trap-silencer tank through a water-cooled heat exchanger, supplied by the seal-water cooling loop, then back to the vacuum pumps. 
The seal-water cooling system consists of two water-to-water heat exchangers and a closed-10op, glycol-cooling subsystem. The closed-10op, water-cooling subsystem is located outside and south of the 234-5Z Building. It consists of two evaporative coolers and two pumps and is powered by a 5-hp electric motor. Filtered water from the process water supply system is evaporated in the evaporative coolers to remove heat from the glycol cooling Toop.

The $43-\mathrm{cm} \mathrm{Hg}$ (17-in. $\mathrm{Hg}$ ) air-sampling vacuum system provides the motive force for continuous air monitor units and fixed-filter air samplers. Pipe headers are run throughout the 234-5Z Building, with branches serving the $291-Z, 242-Z, 2736-Z$, and 232-Z Buildings, to provide the vacuum source. The equipment consists of two vacuum pumps, a moisture separator, and inlet and outlet HEPA filters. The air is collected and filtered by one stage of HEPA filters before entering the vacuum pumps. It is then discharged via the moisture separator and two outlet HEPA filters to the 291-Z Building's exhaust fan inlet plenum.

The PRF has its own complete air monitoring system. Two vacuum pumps, powered by a 25-hp electric motor, are installed in room 35, 236-Z Building. The inlet air to each of the vacuum pumps is passed through a HEPA filter; the outlet air is discharged to the E-3 exhaust system.

\subsubsection{Waste Drainage Piping}

There are three important sections of waste drainage pipe: (1) piping in the tunnels of 234-5Z, (2) piping in the trenches outside of 234-5Z, and (3) piping in Building 241-Z. The drawings used to determine the total volume of waste drainage pipe are Tisted in Table 5-7. The horizontal drainage system was traced on the appropriate drawing. Each drawing has 1 ines representing the drainage piping labeled D-1 through D-8. The given drawing scale was used to determine the length of pipe; the diameters are given on the drawings. The diameter of the main line was used when a pipe branched into a pipe of unknown diameter. When the pipe diameter changed but the exact location of the change was not known, the largest diameter was used to the point at which either the new diameter was given or a tee in the pipe was located.

From the drawings it was determined that the acid drain lines (D-1) exit the 234-5Z Building in two Tocations and join the clear-water drain (D-2) outside of the 234-5Z Building. The combined waste emptied into a drainage ditch southeast of the main building. The process-cooling drain line (D-3) drained into a retention pond approximately $122 \mathrm{~m}$ (400 ft) from the 234-5Z Building. Drains D-4 through D-8 drained into storage tanks located in Building 241-Z. The distance to the storage tanks was assumed to be $91 \mathrm{~m}$ $(300 \mathrm{ft})$. Approximately $23 \mathrm{~m}(75 \mathrm{ft})$ of $15-\mathrm{cm}(6-i n$.$) drainage line connects$ the tanks contained in Building 241-Z with settling tank 241-Z-361 located southeast of the building. A pipe, $46 \mathrm{~m}$ (150 ft) Tong by $20 \mathrm{~cm}$ ( 8 in.) in diameter, exits the settiing tank and drains into three cribs, 216-Z-1, 216-Z-2, and 216-Z-3. A plan view of the pipe system detailing drainage from the five tanks in $241-Z$ to the cribs is shown in Figure 5-1. 
Table 5-7. Drawing List for Pipe Locations.

\begin{tabular}{|c|c|c|}
\hline Location & Drawing number & Drawing type \\
\hline \multirow{5}{*}{$234-5 Z$} & $\begin{array}{l}\mathrm{H}-2-16473 \text { through } \\
\mathrm{H}-2-16480, \mathrm{H}-2-29646\end{array}$ & Duct level plan view \\
\hline & $\mathrm{H}-2-93504$ & Engineering flow diagram, RMC Line \\
\hline & $\mathrm{H}-2-16414, \mathrm{H}-2-16415$ & Below first floor plan view \\
\hline & $\mathrm{H}-2-19325, \mathrm{H}-2-16425$ & First floor plan view \\
\hline & $\mathrm{H}-2-23400$ & Flowcharts \\
\hline $\begin{array}{l}\text { Underground } \\
\text { services }\end{array}$ & $\begin{array}{ll}\mathrm{H}-2-44511, & \mathrm{H}-2-24923 \\
\mathrm{H}-2-16421, & \mathrm{H}-2-23872, \\
\mathrm{H}-2-43517, & \mathrm{H}-2-16420\end{array}$ & Outside services plan view \\
\hline $241-Z$ & H-2-16419 & Waste drains in $241-Z$ \\
\hline \multirow{7}{*}{$236-Z$} & $\mathrm{H}-2-28014$ & Process flowcharts \\
\hline & $\mathrm{H}-2-29657$ & First and second floor plan view \\
\hline & $\mathrm{H}-2-29658$ & Third floor plan view \\
\hline & $\mathrm{H}-2-29658$ & Fourth floor plan view \\
\hline & $\mathrm{H}-2-29659$ & Sectional view \\
\hline & $H-2-29661$ & Chemical preparation room, $\mathrm{plan}$ view \\
\hline & $H-2-29663$ & Equipment room, plan view \\
\hline
\end{tabular}


Figure 5-1. Pipe Plan View Detailing Drainage from 241-Z to the Cribs.

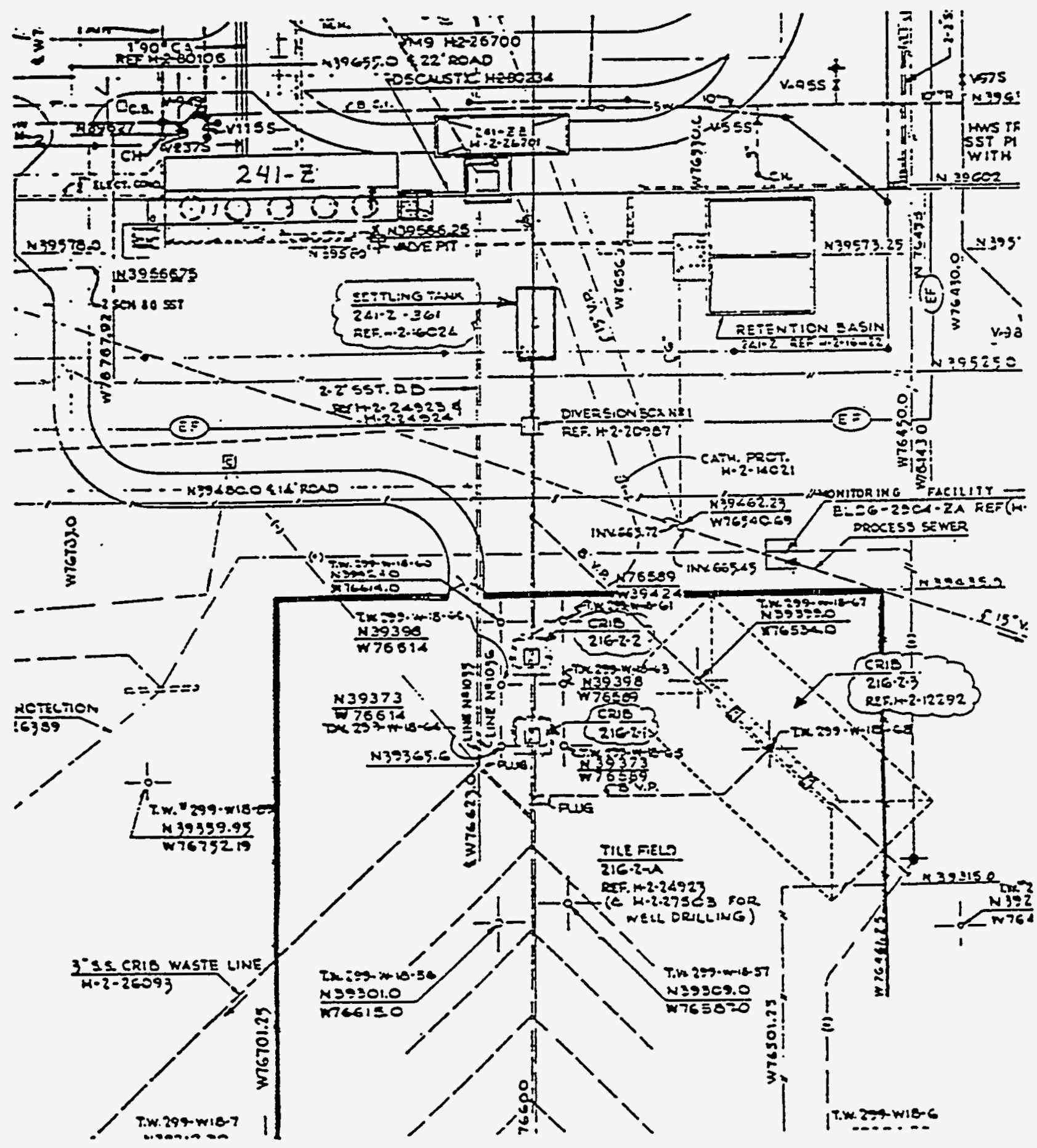


A small trench is located in the front of the 241-Z Building. The trench piping enters the small trench near the mid-section of the building. The pipe then tees off to the appropriate tank. It is assumed that when the lines enter $241-Z$, the D-4 line feeds the D-4 tank, the D-5 line feeds the D-5 tank, and so on.

A11 drainage 1 ines (D-1 through $D-8$ ) are considered TRU MW and have a total estimated volume of $88.5 \mathrm{~m}^{3}$. The volume of drain piping, detailed by line, is listed in Table 5-8. Appendix B, Table B-1, contains dimensional details used to obtain the drain pipe volumes.

\subsubsection{4-5Z Service Piping}

The service piping examined in Building 234-5Z consisted of those 7 ines listed in Table 5-9. The drawings used to determine the total volume of service piping in 234-5Z are listed in Table 5-7. Each system was traced on the appropriate drawing. The length of each system was measured using the given scale. For short branch lines where the diameter was not legible, the diameter was assumed to be the same as the main line. Only the sections of water lines and pressurized air lines, including instrument air, that dropped down into a room were considered TRU. All other sections of water and pressurized air lines were considered uncontaminated. All liquid chemical lines were considered hazardous unless they dropped down into a room. The pipe in the room was considered TRU MW. A11 of the $66-\mathrm{cm} \mathrm{Hg}(26-\mathrm{in}$. $\mathrm{Hg}$ ) vacuum lines and air sampling lines were considered TRU. A 71 gaseous chemical Tines were considered uncontaminated unless they dropped down into a room. The pipe in the room was considered TRU.

The total estimated volume of service piping for $234-5 Z$ is $7.85 \mathrm{~m}^{3}$ TRU, $0.34 \mathrm{~m}^{3} \mathrm{DSW}$, and $0.02 \mathrm{~m}^{3}$ TRU MW. Details by service are given in Table 5-9. Appendix B, Table B-2, contains dimensional details used to obtain service pipe volumes.

\subsubsection{Plutonium Reclamation Facility Waste Drain and Service Piping}

The waste drain service piping examined in Building $236-Z$ consisted of those lines listed in Table 5-10. The drawings used to determine the total volume of service piping in 236-Z are listed in Table 5-7. A11 assumptions used for measuring and classifying the TRU, DSW, and TRU MW piping are the same as those used for measuring and classifying the piping in 234-5Z. In addition, a11 column extractants and column scrubs are considered TRU MH.

The total estimated volume of service piping for $236-Z$ is $0.50 \mathrm{~m}^{3}$ TRU, $1.28 \mathrm{~m}^{3}$ TRU MH, and $0.06 \mathrm{~m}^{3}$ DSH. Details by service are given in Table 5-10. Appendix B, Table B-3, contains dimensional details used to obtain pipe volumes. 
Table 5-8. Drain Pipe Volumes.

\begin{tabular}{|c|c|c|c|}
\hline Drain number & $\begin{array}{c}\text { Tunnel piping } \\
\text { volume } \\
\left(\mathrm{m}^{3}\right)\end{array}$ & $\begin{array}{c}\text { Trench piping } \\
\text { volume } \\
\left(\mathrm{m}^{3}\right)\end{array}$ & $\begin{array}{c}241-\mathrm{z} \text { piping } \\
\text { volume } \\
\left(\mathrm{m}^{3}\right)\end{array}$ \\
\hline D-1 & 0.15 & 29.22 & N/A \\
\hline D-2 & 2.03 & 0.07 & N/A \\
\hline D-1/D-2 & N/A & 28.33 & N/A \\
\hline D-3 & 1.41 & 3.93 & N/A \\
\hline D-4 & 1.67 & 0.79 & 0.02 \\
\hline D-5 & 0.60 & 0.84 & 0.02 \\
\hline D-6 & 4.72 & 5.99 & 0.10 \\
\hline D-7/D-8 & 0.22 & 0.91 & 0.03 \\
\hline Miscellaneous & 0.65 & N/A & 6.77 \\
\hline Total & 11.45 & 70.08 & 6.94 \\
\hline
\end{tabular}


Table 5-9. 234-5Z Service Pipe Volumes.

\begin{tabular}{|c|c|c|c|}
\hline Service piping & $\begin{array}{c}\text { Transuranic } \\
\text { waste } \\
\left(\mathrm{m}^{3}\right)\end{array}$ & $\begin{array}{c}\text { Dangerous } \\
\text { solid waste } \\
\left(\mathrm{m}^{3}\right)\end{array}$ & $\begin{array}{c}\text { Transuranic } \\
\text { mixed waste } \\
\left(\mathrm{m}^{3}\right)\end{array}$ \\
\hline Hot water & 0.025 & N/A & $N / A$ \\
\hline Cold water & 0.063 & $N / A$ & $N / A$ \\
\hline Distilled water & 0.027 & $N / A$ & $N / A$ \\
\hline Chilled water & 0.008 & $N / A$ & $N / A$ \\
\hline G7ycol & $N / A$ & 0.04 & 0.001 \\
\hline Nitric acid (60\%) & N/A & 0.16 & 0.008 \\
\hline Nitric acid $(M)$ & N/A & 0.03 & 0.003 \\
\hline 0xalic acid $(0.67 M)$ & $N / A$ & 0.04 & 0.003 \\
\hline 0xalic acid $(0.1 M)$ & $N / A$ & 0.04 & 0.003 \\
\hline $\mathrm{NaOH}(30 \%)$ & $N / A$ & 0.03 & 0.002 \\
\hline $103-\mathrm{kPa}\left(15-1 \mathrm{~b} / \mathrm{in}^{2}\right)$ air & 0.026 & $N / A$ & $N / A$ \\
\hline $276-\mathrm{kPa}\left(40-1 \mathrm{~b} / \mathrm{in}^{2}\right)$ air & 0.053 & $N / A$ & $N / A$ \\
\hline $621-\mathrm{kPa}\left(90-1 \mathrm{~b} / \mathrm{in}^{2}\right)$ air & 0.020 & $N / A$ & $N / A$ \\
\hline $66-\mathrm{cm} \mathrm{Hg}(26-i n$. $\mathrm{Hg})$ vacuum & 7.44 & $N / A$ & $N / A$ \\
\hline $\begin{array}{l}\text { Instrument air }(207 \mathrm{kPa} \\
\left.\left[301 \mathrm{~b} / \mathrm{in}^{2}\right]\right)\end{array}$ & 0.016 & $N / A$ & $N / A$ \\
\hline Air sampling & 0.097 & $N / A$ & $N / A$ \\
\hline Argon & 0.011 & $N / A$ & N/A \\
\hline Helium & 0.016 & $N / A$ & $N / A$ \\
\hline Propane & 0.024 & $N / A$ & $N / A$ \\
\hline Hydrogen & 0.003 & $N / A$ & $N / A$ \\
\hline Methane & 0.001 & $N / A$ & $N / A$ \\
\hline Methane exhaust & 0.001 & $N / A$ & $N / A$ \\
\hline Nitrogen & 0.009 & $N / A$ & $N / A$ \\
\hline Oxygen & 0.008 & $N / A$ & $N / A$ \\
\hline Hydrofluoric acid & 0.004 & $N / A$ & $N / A$ \\
\hline $\mathrm{SO}_{2}$ & 0.003 & $N / A$ & $N / A$ \\
\hline Total & 7.85 & 0.34 & 0.02 \\
\hline
\end{tabular}


Table 5-10. 236-Z Service Pipe Volumes.

\begin{tabular}{|c|c|c|c|}
\hline Service pipe & $\begin{array}{c}\text { Transuranic } \\
\text { waste } \\
\left(\mathrm{m}^{3}\right)\end{array}$ & $\begin{array}{c}\text { Dangerous } \\
\text { solid } \\
\text { waste } \\
\left(\mathrm{m}^{3}\right)\end{array}$ & $\begin{array}{c}\text { Transuranic } \\
\text { mixed waste } \\
\left(\mathrm{m}^{3}\right)\end{array}$ \\
\hline $\begin{array}{l}\text { Compressed air }(621 \mathrm{kPa} \\
\left.\left[90 \mathrm{lb} / \mathrm{in}^{2}\right]\right)\end{array}$ & 0.013 & $N / A$ & $N / A$ \\
\hline Nitrogen & 0.005 & N/A & $N / A$ \\
\hline Chemical preparation water & $N / A$ & $N / A$ & $N / A$ \\
\hline Instrument air & 0.014 & $N / A$ & $N / A$ \\
\hline Process water & 0.010 & $N / A$ & $N / A$ \\
\hline Protected process water & 0.014 & $N / A$ & $N / A$ \\
\hline Vacuum air sampling & 0.366 & $N / A$ & N/A \\
\hline Dry air & 0.075 & $N / A$ & N/A \\
\hline Aluminum nitrate & N/A & 0.005 & 0.015 \\
\hline Tributyl phosphate & $N / A$ & 0.001 & 0.003 \\
\hline Carbon tetrachloride & $N / A$ & 0.002 & 0.003 \\
\hline Sodium hydroxide & $N / A$ & 0.002 & 0.006 \\
\hline $\begin{array}{l}\text { Chemical addition } \\
\text { (miscellaneous) }\end{array}$ & $N / A$ & 0.017 & 0.003 \\
\hline Dissolver feed & $N / A$ & 0.003 & 0.002 \\
\hline Hydrofluoric acid & $N / A$ & 0.001 & 0.003 \\
\hline Nitric acid & $N / A$ & 0.023 & 0.014 \\
\hline Sodium nitrite & $N / A$ & 0.002 & 0.002 \\
\hline CA column scrub & $N / A$ & $N / A$ & 0.008 \\
\hline CC column extractant & N/A & N/A & 0.004 \\
\hline Co column extractant & N/A & $N / A$ & 0.004 \\
\hline CA column extractant & $N / A$ & $N / A$ & 0.003 \\
\hline $\begin{array}{l}\text { Chemical and clear process } \\
\text { waste drain }\end{array}$ & N/A & $N / A$ & 0.616 \\
\hline Potentially contaminated waste & $N / A$ & $N / A$ & 0.124 \\
\hline Contaminated waste & $N / A$ & $N / A$ & 0.398 \\
\hline Miscell aneous & N/A & $N / A$ & 0.075 \\
\hline Total & 0.50 & 0.06 & 1.28 \\
\hline
\end{tabular}




\subsection{VENTILATION SYSTEM}

All buildings served by the PFP ventilation systems are zoned to ensure confinement of radioactive materials. Zone 1 is designated as those areas where plutonium contamination would not normally be present. Zone 3 consists of areas in which radioactive material is stored or handled in a contained form. Zone 4 consists of the inside of the hoods, gloveboxes, and process cells that may be grossly contaminated because of direct exposure to plutonium. Airflow is from lowest to medium to highest potentially contaminated areas. The ventilation exhaust involved in the $E-3$ and $E-4$ ventilation systems comes from Zone 3 and Zone 4 areas, respectively.

In all buildings, exhaust gases from contaminated and potentially contaminated ventilation zones are filtered via HEPA filters before discharge to the atmosphere. The HEPA filters, as received onsite, are tested by Hanford Environmental Health Foundation personnel to ensure that 99.97 percent of al1 0.3- $\mu \mathrm{m}$-diameter particulates are retained. The HEPA filters are retested before installation to ensure particulate retention and integrity and periodically thereafter in their service locations. Installed differentialpressure instrumentation measures the pressure drop across the filters to ensure that the filter is not plugged or breached. Filters are replaced that do not retain 99.95 percent of the less than $3.0-\mu \mathrm{m}$-diameter test particles during field testing.

The HEPA filter inlet air is treated as necessary to prevent moisture accumulation within the filters. Vent exhaust is heated as necessary to preclude HEPA filter wetting.

Air from known-contamination ventilation exhaust zones (Zone 4 ) is filtered by at least two stages of testable HEPA filtration before discharge to the atmosphere via a stack ( $E-4$ ventilation exhaust system). The $E-4$ exhaust systems normally contain HEPA filters on glovebox or cell exhausts (see Section 5.1, Table 5-1), with the first testable HEPA filter located in a filterbox (see Tables 5-11 and 5-12). Exhaust flows from these filters are manifolded to a room containing a bank of testable HEPA filters (second stage) before discharge to the atmosphere via a stack.

Table 5-11 provides a 1ist of PFP HEPA filters, both glovebox HEPA filters and testable HEPA filters, and includes their locations by building and room number, dimensions, volumes, and the best estimates of plutonium holdup. The number and volume of HEPA filters (assumed to be removed from filterboxes before D\&D activities) are important because, at this time, the Waste Isolation Pilot Plant will not accept them. There are 1,760 HEPA filters (353 glovebox filters and 1407 testable filters) with a total volume of $154 \mathrm{~m}^{3}$. All glovebox filters are assumed to be standard size $(20 \times 20 \times 15 \mathrm{~cm}[8 \times 8 \times 6$ in.]). The filters in filter rooms 309 and 310 are contaminated by nitric crystals (various nitrogen salts) that originated from the use in the process of nitric acid, which vaporized and passed through the ventilation system. Of the total waste expected from HEPA filters, 90 percent is expected to be TRU MW because of the presence of dioctyl phthalate, a state-regulated compound used during filter efficiency testing; the remaining 10 percent is expected to be TRU. 
Table 5-11. Volume of High-Efficiency Particulate Air Filters. (3 sheets)

\begin{tabular}{|c|c|c|c|c|c|c|c|c|c|}
\hline \multirow[t]{2}{*}{$\begin{array}{l}\text { Building } \\
\text { number }\end{array}$} & \multirow[t]{2}{*}{ Room number } & \multirow{2}{*}{$\begin{array}{l}\text { Number of } \\
\text { glovebox } \\
\text { HEPA } \\
\text { filters }\end{array}$} & \multirow{2}{*}{$\begin{array}{c}\text { Number } \\
\text { of } \\
\text { testable } \\
\text { filters }\end{array}$} & \multirow{2}{*}{$\begin{array}{l}\text { Plutonium } \\
\text { holdup } \\
\text { (g) }\end{array}$} & \multicolumn{3}{|c|}{$\begin{array}{l}\text { Dimensions } \\
\text { (m) }\end{array}$} & \multirow{2}{*}{$\begin{array}{c}\text { Volume } \\
\left(m^{3}\right)\end{array}$} & \multirow{2}{*}{$\begin{array}{c}\text { Information } \\
\text { origin }\end{array}$} \\
\hline & & & & & Length & Depth & Diameter & & \\
\hline $216-2-9 B$ & Bldg. & $\cdots$ & 2 & -- & 0.61 & 0.29 & -- & 0.22 & Drawings \\
\hline \multirow{2}{*}{$232-2$} & \multirow{2}{*}{ Bldg. } & $\cdots$ & 6 & - & 0.61 & 0.29 & $\cdots$ & 0.65 & Drawings \\
\hline & & -- & 6 & $\cdots$ & 0.61 & 0.51 & -- & 1.13 & Drawings \\
\hline $234-5 z$ & 131 & 9 & $\cdots$ & 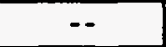 & 0.20 & 0.20 & -- & 0.06 & 23400 \\
\hline \multirow{2}{*}{$234-52$} & \multirow{2}{*}{134} & -- & 2 & $\cdots$ & 0.30 & 0.29 & -- & 0.05 & Drawings \\
\hline & & 4 & $\cdots$ & -- & 0.20 & 0.20 & $\cdots$ & 0.03 & 23400 \\
\hline $234-52$ & 136 & 8 & $\cdots$ & $\cdots$ & 0.20 & 0.20 & -- & 0.05 & 23400 \\
\hline $234-52$ & 137 & 12 & -- & $\cdots$ & 0.20 & 0.20 & -- & 0.08 & 23400 \\
\hline $234-5 z$ & 139 & 3 & $\overline{--}$ & -- & 0.20 & 0.20 & -- & 0.02 & 23400 \\
\hline $234-52$ & 145 & 2 & -- & -- & 0.20 & 0.20 & $\cdots$ & 0.01 & 23400 \\
\hline \multirow{2}{*}{$234-52$} & \multirow{2}{*}{146} & -- & 4 & -- & 0.30 & 0.29 & $\cdots$ & 0.11 & Drawings \\
\hline & & 9 & -- & $\cdots$ & 0.20 & 0.20 & -- & 0.06 & 23400 \\
\hline \multirow{3}{*}{$234-52$} & \multirow{3}{*}{149} & -- & 1 & -- & 0.61 & 0.29 & -- & 0.11 & Drawings \\
\hline & & -- & 1 & $\cdots$ & 0.30 & 0.29 & -- & 0.05 & Drawings \\
\hline & & 4 & $\cdots$ & $\cdots$ & 0.20 & 0.20 & -- & 0.03 & 23400 \\
\hline $234-52$ & 152 & 6 & -- & - & 0.20 & 0.20 & -- & 0.04 & $\cdots$ \\
\hline $234-5 z$ & 153 & 4 & -- & -- & 0.20 & 0.20 & -- & 0.03 & 23400 \\
\hline $234-52$ & 154 & 11 & $\cdots$ & - & 0.20 & 0.20 & - & 0.07 & 23400 \\
\hline \multirow{2}{*}{$234-52$} & \multirow{2}{*}{156} & $\cdots$ & 1 & $\cdots$ & 0.30 & 0.15 & $\cdots$ & 0.01 & Drawings \\
\hline & & 3 & -- & -- & 0.20 & 0.20 & -- & 0.02 & 23400 \\
\hline \multirow{2}{*}{$234-5 z$} & \multirow{2}{*}{157} & -- & 2 & -- & 0.30 & 0.15 & $\cdots$ & 0.03 & Drawings \\
\hline & & 6 & -- & $\cdots$ & 0.20 & 0.20 & - & 0.04 & 23400 \\
\hline $234-52$ & 166 & 7 & $\cdots$ & $\cdots$ & 0.20 & 0.20 & -- & 0.04 & 23400 \\
\hline $234-52$ & 169 & 2 & -- & -- & 0.20 & 0.20 & $\cdots$ & 0.01 & 23400 \\
\hline $234-52$ & 170 & 3 & $\cdots$ & $-\cdot$ & 0.20 & 0.20 & -- & 0.02 & 23400 \\
\hline $234-52$ & 179 & 48 & -- & - & 0.20 & 0.20 & -- & 0.30 & 23400 \\
\hline \multirow{2}{*}{$234-52$} & \multirow{2}{*}{180} & $\cdots$ & 2 & $\cdots$ & 0.30 & 0.29 & -- & 0.05 & Drowings \\
\hline & & 2 & $\cdots$ & $\cdots$ & 0.20 & 0.20 & $\cdots$ & 0.01 & 23400 \\
\hline $234-52$ & 185 & 4 & $\ldots$ & -- & 0.20 & 0.20 & -- & 0.03 & 23400 \\
\hline $234-52$ & 188 & 7 & -- & $\cdots$ & 0.20 & 0.20 & $\cdots$ & 0.04 & 23400 \\
\hline \multirow{4}{*}{$234-52$} & & $\cdots$ & 4 & -- & 0.30 & 0.29 & $\cdots$ & 0.11 & Drawings \\
\hline & $221-C$ & -- & 2 & $\cdots$ & 0.61 & 0.29 & $\cdots$ & 0.22 & Drowings \\
\hline & & -- & 1 & -- & 0.20 & 0.15 & $\cdots$ & 0.01 & Drawings \\
\hline & & 11 & $\cdots$ & -- & 0.20 & 0.20 & -- & 0.07 & 23400 \\
\hline & & $\cdots$ & 4 & $\cdots$ & 0.61 & 0.29 & - & 0.43 & Drawings \\
\hline $234-52$ & $22 i-D$ & $\cdots$ & 3 & $\cdots$ & 0.30 & 0.29 & $\cdots$ & 0.16 & Drawings \\
\hline & & -- & 1 & $\cdots$ & 0.20 & 0.15 & $\cdots$ & 0.01 & Drawings \\
\hline & & 13 & $\cdots$ & $\cdots$ & 0.20 & 0.20 & $\cdots$ & 0.08 & 23400 \\
\hline $234-5 z$ & $221-E$ & -- & 3 & $\cdots$ & 0.61 & 0.29 & $\cdots$ & 0.33 & Drawings \\
\hline & & 7 & $\cdots$ & -- & 0.20 & 0.20 & -- & 0.04 & 23400 \\
\hline $234-52$ & 227 & 4 & $\cdots$ & $\cdots$ & 0.20 & 0.20 & $\cdots$ & 0.03 & 23400 \\
\hline
\end{tabular}


Table 5-11. Volume of High-Efficiency Particulate Air Filters. (3 sheets)

\begin{tabular}{|c|c|c|c|c|c|c|c|c|c|}
\hline \multirow[t]{2}{*}{$\begin{array}{l}\text { Building } \\
\text { number }\end{array}$} & \multirow[t]{2}{*}{ Room number } & \multirow{2}{*}{$\begin{array}{l}\text { Number of } \\
\text { glovebox } \\
\text { HEPA } \\
\text { filters }\end{array}$} & \multirow{2}{*}{$\begin{array}{c}\text { Number } \\
\text { of } \\
\text { testable } \\
\text { filters }\end{array}$} & \multirow{2}{*}{$\begin{array}{c}\text { Plutonium } \\
\text { holdup } \\
\text { (g) }\end{array}$} & \multicolumn{3}{|c|}{$\begin{array}{l}\text { Dimensions } \\
\text { (m) }\end{array}$} & \multirow{2}{*}{$\begin{array}{c}\text { Volyme } \\
\left(m^{3}\right)\end{array}$} & \multirow{2}{*}{$\begin{array}{l}\text { Information } \\
\text { origin }\end{array}$} \\
\hline & & & & & Length & Depth & Diameter & & \\
\hline \multirow{2}{*}{$234-52$} & \multirow{2}{*}{$228-A$} & $\cdots$ & 2 & $\cdots$ & $\cdots$ & $\cdots$ & 0.19 & 0.01 & Drawings \\
\hline & & 15 & $\cdots$ & -- & 0.20 & 0.20 & $\cdots$ & 0.09 & 23400 \\
\hline $234-52$ & $228-B$ & 4 & -- & $\cdots$ & 0.20 & 0.20 & -- & 0.03 & 23400 \\
\hline $234-52$ & $228-C$ & 10 & $\cdots$ & -- & 0.20 & 0.20 & -- & 0.06 & 23400 \\
\hline $234-52$ & $230-A$ & 1 & $\cdots$ & -- & 0.20 & 0.20 & -- & 0.01 & 23400 \\
\hline $234-52$ & $230-8$ & 3 & $\cdots$ & $\cdots$ & 0.20 & 0.20 & -- & 0.02 & 23400 \\
\hline $234-5 z$ & $230-c$ & 3 & $\cdots$ & 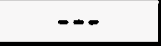 & 0.20 & 0.20 & -- & 0.02 & 23400 \\
\hline $234-52$ & 232 & 7 & -- & -- & 0.20 & 0.20 & -- & 0.04 & 23400 \\
\hline \multirow{2}{*}{$234-52$} & \multirow{2}{*}{234} & $\cdots$ & 1 & $\cdots$ & 0.61 & 0.29 & $\cdots$ & 0.11 & Drawings \\
\hline & & 1 & -- & -- & 0.20 & 0.20 & $\cdots$ & 0.01 & 23400 \\
\hline $234-52$ & 235-A1 & 2 & -- & -- & 0.20 & 0.20 & -- & 0.01 & 23400 \\
\hline $234-5 z$ & 235-A2 & 7 & $\cdots$ & -- & 0.20 & 0.20 & -- & 0.04 & 23400 \\
\hline $234-52$ & $235-A 3$ & 10 & -- & -- & 0.20 & 0.20 & $\cdots$ & 0.06 & 23400 \\
\hline $234-52$ & $235-B$ & 16 & $\cdots$ & $\cdots$ & 0.20 & 0.20 & -- & 0.10 & 23400 \\
\hline \multirow{5}{*}{$234-52$} & \multirow{5}{*}{ 235-D } & - & 3 & -- & 0.61 & 0.51 & $\cdots$ & 0.57 & Drawings \\
\hline & & $\cdots$ & 1 & -- & 0.61 & 0.29 & -- & 0.11 & Drawings \\
\hline & & -- & 3 & -- & 0.30 & 0.95 & -- & 0.04 & Drawings \\
\hline & & - & 2 & -- & 0.61 & 0.36 & $\cdots$ & 0.26 & Drawings \\
\hline & & 15 & -- & -- & 0.20 & 0.20 & $\cdots$ & 0.09 & 23400 \\
\hline $234-52$ & 235-E & 2 & -- & -- & 0.20 & 0.20 & $\cdots$ & 0.01 & 23400 \\
\hline \multirow{3}{*}{$234-52$} & \multirow{3}{*}{262} & -- & 16 & $\cdots$ & 0.61 & 0.29 & -- & 1.74 & Drawings \\
\hline & & -- & 2 & -- & 0.61 & 0.29 & $\cdots$ & 0.22 & Drawings \\
\hline & & 2 & -- & $\cdots$ & 0.20 & 0.20 & -- & 0.01 & 23400 \\
\hline \multirow{2}{*}{$234-5 z$} & \multirow{2}{*}{263} & 3 & $\cdots$ & -- & 0.20 & 0.20 & -- & 0.02 & 23400 \\
\hline & & $\cdots$ & 14 & 36 & 0.61 & 0.29 & $\cdots$ & 1.52 & Drawings \\
\hline \multirow{3}{*}{$234-5 z$} & \multirow{3}{*}{264} & -- & 4 & 46 & 0.61 & 0.29 & $\cdots$ & 0.43 & Drawings \\
\hline & & $\cdots$ & 3 & 20 & 0.61 & 0.15 & -- & 0.17 & Drawings \\
\hline & & 2 & $\cdots$ & $\cdots$ & 0.20 & 0.20 & $\cdots$ & 0.01 & 23400 \\
\hline $234-52$ & 270 & $\cdots$ & 2 & -- & 0.61 & 0.29 & -- & 0.22 & Drawings \\
\hline $234-52$ & 308 & -- & 107 & $\cdots$ & 0.61 & 0.29 & $\cdots$ & 11.61 & Drawings \\
\hline $234-52$ & 309 (filter rm) & -- & 128 & $\cdots$ & 0.61 & 0.29 & $-\cdot$ & 13.89 & Drawings \\
\hline $234-52$ & 310 (filter $\mathrm{rm}$ ) & -- & 128 & $\cdots$ & 0.61 & 0.29 & $\cdots$ & 13.89 & Drawings \\
\hline $234-52$ & 311 (filter $\mathrm{rm}$ ) & $\cdots$ & 112 & -- & 0.61 & 0.29 & -- & 12.16 & Drauings \\
\hline $234-52$ & 312 (filter rm) & -- & 112 & -- & 0.61 & 0.29 & -- & 12.16 & Drawings \\
\hline $234-52$ & 313 (filter rm) & -- & 112 & $\cdots$ & 0.61 & 0.29 & $\cdots$ & 12.16 & Drawings \\
\hline $234-52$ & 314 (filter rm) & $\cdots$ & 112 & -- & 0.61 & 0.29 & $\cdots$ & 12.16 & Drawings \\
\hline $234-52$ & 315 (filter $\mathrm{rm}$ ) & $\cdots$ & 112 & $\cdots$ & 0.61 & 0.29 & $\cdots$ & 12.16 & Drawings \\
\hline $234-5 z$ & 316 (filter $\mathrm{rm}$ ) & -- & 112 & $\because$ & 0.61 & 0.29 & - & 12.16 & Drauings \\
\hline $234-52$ & 318 (filter $\mathrm{rm}$ ) & -- & 102 & $-\cdot$ & 0.61 & 0.29 & -- & 11.07 & Drawings \\
\hline $234-5 z$ & 320 & -- & 12 & -- & 0.61 & 0.29 & -- & 1.30 & Drawings \\
\hline
\end{tabular}


Table 5-11. Volume of High-Efficiency Particulate Air Filters. ( 3 sheets)

\begin{tabular}{|c|c|c|c|c|c|c|c|c|c|}
\hline \multirow[t]{2}{*}{$\begin{array}{l}\text { Building } \\
\text { number }\end{array}$} & \multirow[t]{2}{*}{ Room number } & \multirow{2}{*}{$\begin{array}{c}\text { Number of } \\
\text { glovebox } \\
\text { HEPA } \\
\text { filters }\end{array}$} & \multirow{2}{*}{$\begin{array}{c}\text { Number } \\
\text { of } \\
\text { testable } \\
\text { filters }\end{array}$} & \multirow{2}{*}{$\begin{array}{c}\text { Plutonium } \\
\text { holdup } \\
(g)\end{array}$} & \multicolumn{3}{|c|}{$\begin{array}{l}\text { Dimensions } \\
\text { (m) }\end{array}$} & \multirow{2}{*}{$\begin{array}{c}\text { Volume } \\
\left(m^{3}\right)\end{array}$} & \multirow{2}{*}{$\begin{array}{l}\text { Information } \\
\text { origin }\end{array}$} \\
\hline & & & & & Length & Depth & Oiameter & & \\
\hline \multirow{2}{*}{$236-Z$} & \multirow{2}{*}{19} & $\cdots$ & 1 & $\cdots$ & 0.61 & 0.29 & -- & 0.11 & Drawings \\
\hline & & 1 & $-\cdots$ & -- & 0.20 & 0.20 & $\cdots$ & 0.01 & 29674 \\
\hline \multirow{2}{*}{$236-2$} & \multirow{2}{*}{20} & -- & 7 & -- & 0.61 & 0.29 & -- & 0.76 & Drawings \\
\hline & & 3 & -- & -- & 0.20 & 0.20 & -- & 0.02 & 29674 \\
\hline $236-z$ & 25 & 1 & -- & $\cdots$ & 0.20 & 0.20 & -- & 0.01 & 29674 \\
\hline $236-2$ & 26 & -- & 48 & 9 & 0.61 & 0.29 & -- & 5.21 & Drawings \\
\hline $236-z$ & 27 & 2 & -- & -- & 0.20 & 0.20 & -- & 0.01 & 29674 \\
\hline \multirow{2}{*}{$236-2$} & \multirow{2}{*}{30} & -- & 4 & -- & 0.61 & 0.29 & $\cdots$ & 0.43 & Drawings \\
\hline & & 4 & $\cdots$ & -- & 0.20 & 0.20 & - & 0.03 & 29674 \\
\hline $236-2$ & 35 & -- & 2 & -- & 0.61 & 0.29 & -- & 0.22 & Drawings \\
\hline $236-2$ & 36 & -- & 1 & $\cdots$ & 0.61 & 0.29 & $\cdots$ & 0.11 & Drawings \\
\hline $236-2$ & 41 & 39 & -- & 85 & 0.20 & 0.20 & $\cdots$ & 0.25 & 28674 \\
\hline $236-2$ & 43 & 7 & -- & -- & 0.20 & 0.20 & -- & 0.04 & 29674 \\
\hline $236-2$ & 50 & -- & 2 & -- & 0.61 & 0.29 & -- & 0.22 & Drawings \\
\hline $236-2$ & 60 & 4 & $\cdots$ & -- & 0.20 & 0.20 & $\cdots$ & 0.03 & 29674 \\
\hline $241-2$ & Bldg. & -- & 4 & 3 & 0.61 & 0.29 & -- & 0.43 & Drawings \\
\hline $291-2$ & 502 & -- & 16 & -- & 0.61 & 0.51 & $\cdots$ & 3.02 & Drawings \\
\hline $2736-2 A$ & Bldg. & - & 26 & -- & 0.61 & 0.29 & -- & 2.82 & Drawings \\
\hline $2736-2 B$ & 600 & $\cdots$ & 33 & -- & 0.61 & 0.29 & $\cdots$ & 3.58 & Drawings \\
\hline $2736-2 B$ & 630 & $\cdots$ & 1 & -- & 0.61 & 0.29 & -- & 0.11 & Drawings \\
\hline $2736-2 B$ & 636 & $\cdots$ & 2 & -- & 0.30 & 0.15 & -- & 0.03 & Drawings \\
\hline $2736-2 B$ & 637 & $\cdots$ & 8 & $\cdots$ & 0.61 & 0.29 & -- & 0.87 & Drawings \\
\hline $2736-28$ & 638 & - & 2 & -- & 0.61 & 0.29 & $\cdots$ & 0.22 & Drawings \\
\hline $2736-2 B$ & 639 & -- & 1 & $\cdots$ & 0.61 & 0.29 & -- & 0.11 & Drawings \\
\hline $2736-2 B$ & 641 & -- & 2 & -- & 0.61 & 0.29 & -- & 0.22 & Drawings \\
\hline $2736-2 B$ & 642 & $\cdots$ & 2 & $\cdots$ & 0.61 & 0.29 & -- & 0.22 & Drawings \\
\hline & tals & 353 & 1,407 & 199 & & Total & volume & 154.47 & -- \\
\hline
\end{tabular}


The filterboxes that house the testable HEPA filters, with the exception of those in filter rooms, are listed by location in Table 5-12. Their volumes were calculated from their overall dimensions. These filterboxes account for $100 \mathrm{~m}^{3}$ of TRU waste. Dimensions are from various facility drawings

(H-2-131559 in particular). Both Tables 5-11 and 5-12 were verified during the walkdown.

For Building 234-5Z, ventilation supply air is provided to meet the criteria of limiting release of radioactive contaminants to the environment and of minimizing the spread of contamination. Five supply fans are normaliy used to provide proper balancing of the individual air zones. Three fans provide backup for normal fan maintenance activities. The supply fans draw air from outside the building through roughing filters to remove airborne dust and dirt. The air then passes through preheaters, air washers, and reheaters before it goes to the supply plenum. The year-round temperature of the air is approximately $20^{\circ} \mathrm{C}\left(70^{\circ} \mathrm{F}\right)$. Ducts lead from the supply plenum chamber to the various building zones ventilated by the system.

In Building 234-5Z, exhaust air from Zone 3 areas is filtered through a single stage of testable HEPA filters located in seven filter rooms. Exhaust air from Zone 4 areas, which are potentially contaminated or known to be contaminated, is routed to a single stage of testable HEPA filtration with individual filters or to several filters operated in parallel. Exhaust from these filters is manifolded and routed to the E-4 filter rooms, which provide a second stage of testable HEPA filtration via filter banks. The E-4 ventilation exhaust is then combined with the E-3 exhaust downstream of the filters and discharged to the atmosphere via the 291-Z-1 stack. The E-4 system exhaust flow is maintained at -12.7 to $-50.8 \mathrm{~mm} \mathrm{w} . \mathrm{g} .(-0.5$ to -2.0 in. w.g.) to minimize the potential for backflow into Zone 3 areas; similarly, the E-3 system exhaust flow is maintained at -3.8 to $-12.7 \mathrm{~mm} \mathrm{w.g}$. $(-0.15$ to -0.5 in. w.g. $)$ to prevent backflow into Zone 1 areas.

For Building $241-Z$, one exhaust ventilation system with two branches serves both the 241-Z tanks and cells. Exhaust ventilation from the tanks is routed via the vessel vent system, which consists of a 10.2-cm- (4-in.-) diameter header with tie-ins to each tank, and a demister. A valve in the header, upstream of the demister, controls the vessel vent exhaust flow to $2.83 \mathrm{~m}^{3} / \mathrm{min}\left(100 \mathrm{~m}^{3} / \mathrm{min}\right)$. The vessel vent system exhaust joins the cell ventilation exhaust downstream of the demister. The 241- $Z$ tank sampler glovebox and D-9 caustic supply tank are also exhausted via the vessel vent system. Exhaust ventilation from the $241-Z$ cells is routed via a $38.1-\mathrm{cm}-$ (15-in.-) diameter vitreous clay duct. Exhaust air from the two systems is heated to protect the HEPA filters from moisture buildup and routed through two stages of HEPA filtration, in series, before discharge to the atmosphere via the stainless steel stack.

The 2736-ZA Building consists of two rooms. Room 1 houses a diesel generator that provides emergency power for operation of the EF-I-1 and EF-1-2 exhaust fans and associated instrumentation and controls, which are housed in room 2. Room 2 is exhausted to the atmosphere via two stages of HEPA filtration and exhaust fans. Exhaust ventilation for room 1 is provided by wall louvers and a through-the-wall motorized exhaust fan. 


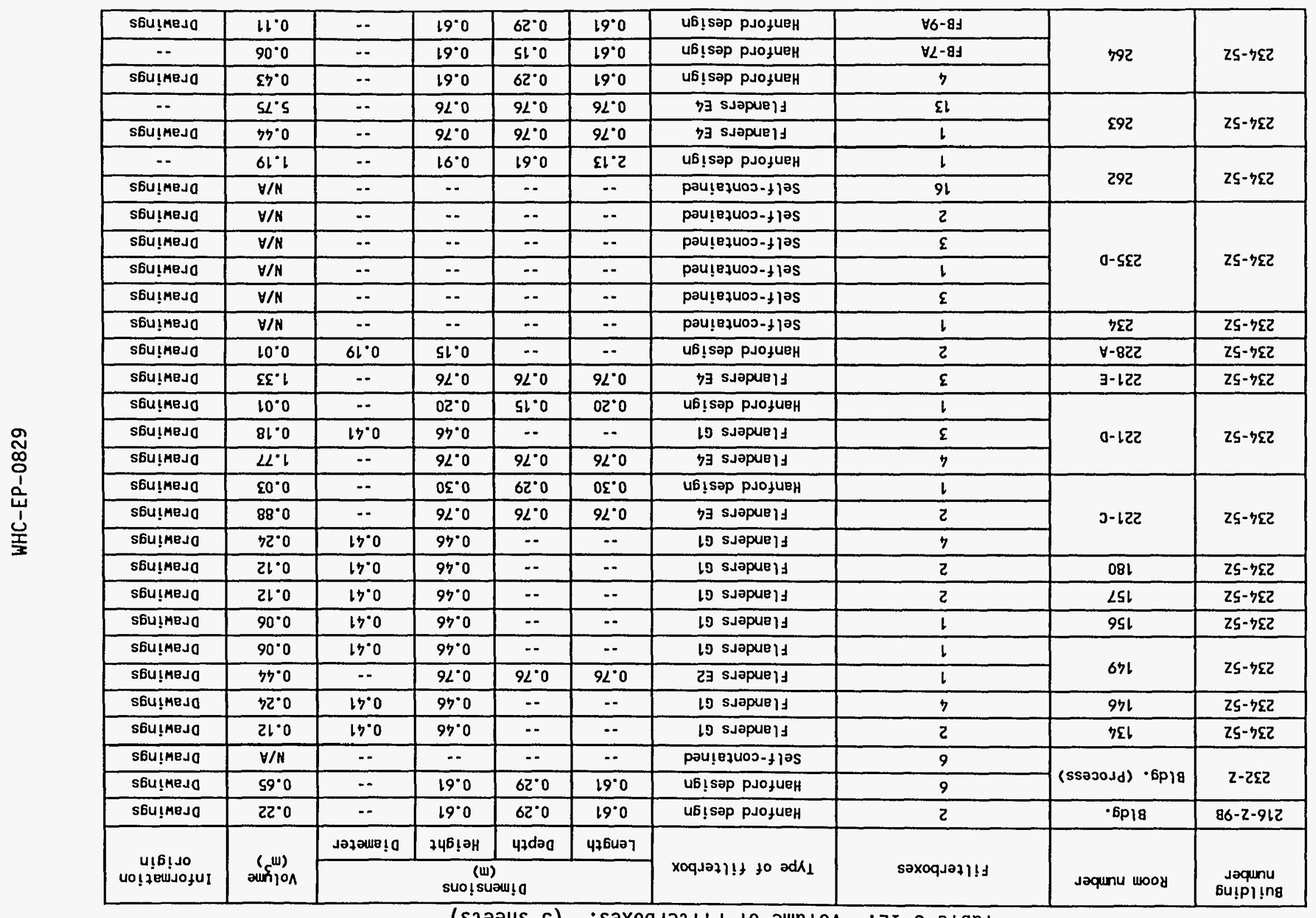


Table 5-12. Volume of Filterboxes. (3 sheets)

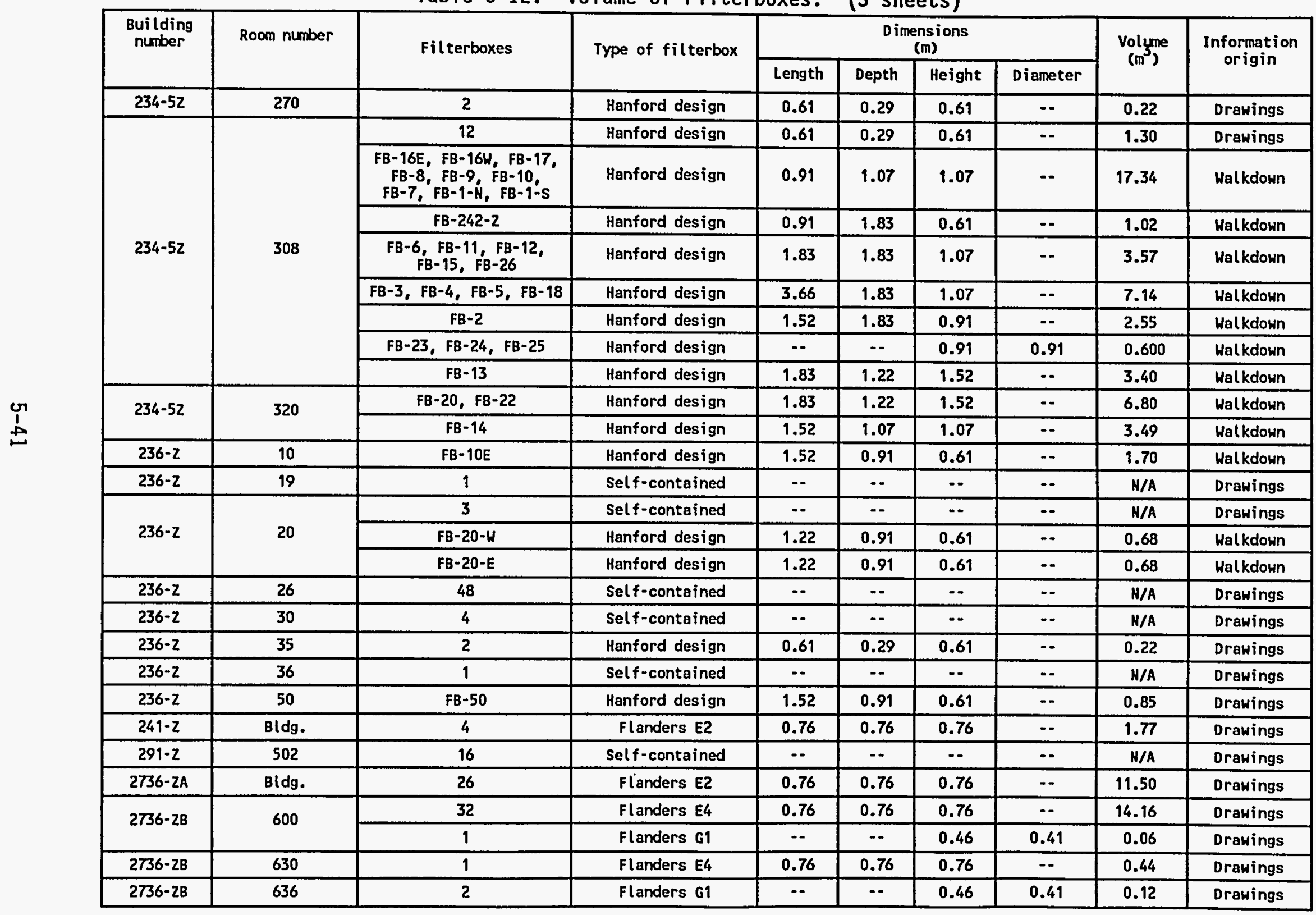


Table 5-12. Volume of Filterboxes. (3 sheets)

\begin{tabular}{|c|c|c|c|c|c|c|c|c|c|}
\hline \multirow[t]{2}{*}{$\begin{array}{l}\text { Building } \\
\text { number }\end{array}$} & \multirow[t]{2}{*}{ Room number } & \multirow[t]{2}{*}{ Fil terboxes } & \multirow[t]{2}{*}{ Type of filterbox } & \multicolumn{4}{|c|}{$\begin{array}{c}\text { Dimensions } \\
\text { (m) }\end{array}$} & \multirow[t]{2}{*}{$\begin{array}{l}\text { Volume } \\
\left(m^{3}\right)\end{array}$} & \multirow[t]{2}{*}{$\begin{array}{c}\text { Information } \\
\text { origin }\end{array}$} \\
\hline & & & & Length & Depth & Height & Diameter & & \\
\hline $2736-2 B$ & 637 & 8 & Flanders E4 & 0.76 & 0.76 & 0.76 & -- & 3.54 & Drawings \\
\hline $2736-2 B$ & 638 & 2 & Flanders E4 & 0.76 & 0.76 & 0.76 & -- & 0.88 & Drawings \\
\hline $2736-2 B$ & 639 & 1 & Flanders E4 & 0.76 & 0.76 & 0.76 & $\cdots$ & 0.44 & Drawings \\
\hline $2736-28$ & 641 & 2 & Flanders E4 & 0.76 & 0.76 & 0.76 & $\therefore$ & 0.88 & Drawings \\
\hline $2736-2 B$ & 642 & 2 & Flanders E4 & 0.76 & 0.76 & 0.76 & $\therefore$ & 0.88 & Drawings \\
\hline & & & & & & \multicolumn{2}{|c|}{ Total volume } & 100.46 & \\
\hline
\end{tabular}


Ventilation air for the 2736-ZB Building is supplied by two systems. One system provides conditioned air via two supply fans to all areas of the building except that portion housing the NDA laboratory. Exhaust air from Zone 4 is filtered via a single-stage HEPA filter, combined with Zone 3 exhaust air and filtered through two additional stages of HEPA filtration, then discharged to the atmosphere via the 296-Z-5 stack. The second ventilation supply system provides filtered, conditioned air to the NDA laboratory portion of the building. The supply air is maintained at the required temperature by steam heating during the winter and refrigeration cooling during the warmer seasons. Exhaust air is collected by three exhaust ducts, each provided with a single stage of HEPA filtration. These exhaust flows are combined, filtered via two additional stages of HEPA filtration, and recycled to the NDA supply system by fans. Recycled air is diverted to provide supply air for the mechanical equipment and is continuously monitored and sampled for radioactive content.

\subsubsection{4-5Z Supply Systems}

The drawings used to determine the total volume of the air supply system in 234-5Z are 1isted in Table 5-13. The air supply system was broken down into five sections, $S-1, S-2, S-3, S-3 A$, and $S-5$. The $S-1$ air supply system was considered uncontaminated; therefore the volume was not determined. The volumes of the $S-2, S-3$, and $S-3 A$ air supply systems were determined using the cross-sectional areas and the lengths of the duct work. The cross-sectional areas were determined using the isometric drawings, and the lengths were measured from the plan drawings. From the isometric drawings of the $\mathrm{S}-5$ air supply systems, it was determined that the system occupies only a small area near room 192. It was estimated that the total volume of the S-5 air supply system is approximately 1 percent of the total volume for all air supply systems in 234-5Z.

A precise measurement, consisting of all elevation changes and bends in duct work, was made for a portion of the S-2 air supply system. The precise measurement was compared to the estimated value. The precise measurement was approximately 10 percent greater then the estimated value. From this information, it could be stated that the volume of the combined air supply systems could be underestimated by 10 percent. The total volume of air supply system duct work in 234-5Z is $1632 \mathrm{~m}^{3}$ (see Table 5-14). The S-2, S-3, S-3A, and S-5 air supply systems are considered LLW. It is feasible to assume that contamination of the air supply systems could have occurred because of backflow of air during system shutdown.

\subsubsection{4-5Z E-3 and E-4 Exhaust}

The drawings used to determine the total volume of $E-3$ and $E-4$ exhaust ducting in Building 234-5Z are listed in Table 5-13. The E-3 exhaust ducts occupying the first floor and duct level of the PFP Building were highlighted on the plan drawings then compared to the isometric drawings of the ventilation layout to determine the cross-sectional area of the ducts. The length of the ducts was measured from the plan view of the ventilation system. The volume was then calculated for the horizontal duct work. The volume of vertical duct work was determined using the cross-sectional area and an 
Tab7e 5-13. Reference Drawing List.

\begin{tabular}{|l|l|l|}
\hline Building & \multicolumn{1}{|c|}{ Drawing number } & \multicolumn{1}{|c|}{ Drawing type } \\
\hline $234-5 Z$ & $\begin{array}{l}\mathrm{H}-2-16429 \text { through } \mathrm{H}-2-16432, \\
\mathrm{H}-2-80203\end{array}$ & P1an views \\
\cline { 2 - 3 } & $\mathrm{H}-2-16435$ through $\mathrm{H}-2-16447$ & Sectional views \\
\cline { 2 - 3 } & $\mathrm{H}-2-24606$ & Isometric (S-2) \\
\cline { 2 - 3 } & $\mathrm{H}-2-24607$ & Isometric (S-3) \\
\cline { 2 - 3 } & $\mathrm{H}-2-24608$ & Isometric (S-3A) \\
\hline & $\mathrm{H}-2-24609$ & Isometric (S-5) \\
\cline { 2 - 3 } & $\mathrm{H}-2-26060$ through H-2-26062 & Isometric (E-3) \\
\cline { 2 - 3 } & $\mathrm{H}-2-26063$ through H-2-26066 & Isometric (E-4) \\
\hline $236-\mathrm{Z}$ & $\mathrm{H}-2-29675, \mathrm{H}-2-29676$, & \\
\hline $241-\mathrm{Z}$ & $\mathrm{H}-2-29677$ & \\
\hline $2736-\mathrm{ZA}$ & $\mathrm{H}-2-28851$ & \\
\hline $2736-\mathrm{ZB}$ & $\mathrm{H}-2-80168, \mathrm{H}-2-80169$ & \\
\hline
\end{tabular}

Table 5-14. Ventilation System Volume.

\begin{tabular}{|c|c|c|c|c|}
\hline \multirow[b]{2}{*}{ Building } & \multirow{2}{*}{$\underset{\left(\mathrm{m}^{3}\right)}{\text { Building volume }}$} & \multirow{2}{*}{$\begin{array}{c}\begin{array}{c}\text { Supply system } \\
\text { volume }\left(\mathrm{m}^{3}\right)\end{array} \\
\begin{array}{c}\text { Low-level } \\
\text { waste }\end{array}\end{array}$} & \multicolumn{2}{|c|}{$\begin{array}{c}\text { Exhaust volume } \\
\left(\mathrm{m}^{3}\right)\end{array}$} \\
\hline & & & $\begin{array}{c}\text { Low-level } \\
\text { waste }\end{array}$ & $\begin{array}{c}\text { Transuranic } \\
\text { waste }\end{array}$ \\
\hline $234-5 Z$ & 92,511 & 1,632 & 2,081 & 23 \\
\hline $236-Z$ & 7,624 & 99 & 28 & 3 \\
\hline $241-Z$ & 625 & $\begin{array}{c}\text { Not } \\
\text { significant }\end{array}$ & $\begin{array}{c}\text { Not } \\
\text { significant }\end{array}$ & $\begin{array}{c}\text { Not } \\
\text { significant }\end{array}$ \\
\hline $2736-Z A$ & 600 & $\begin{array}{c}\text { Not } \\
\text { significant }\end{array}$ & $\begin{array}{c}\text { Not } \\
\text { significant }\end{array}$ & $\begin{array}{c}\text { Not } \\
\text { significant }\end{array}$ \\
\hline $2736-Z B$ & 4,037 & 43 & 30 & 0 \\
\hline Total & 105,397 & 1,774 & 2,139 & 26 \\
\hline
\end{tabular}

*ess then 1 percent of the total volume.

assumed length of $3 \mathrm{~m}(10 \mathrm{ft})$. The assumed length was determined by averaging the lengths of the vertical ducts for room ventilation on the sectional drawings. The cross-sectional areas of all vertical duct work were given on the isometric drawings. A total volume of horizontal duct work and vertical duct work was calculated. 
To determine the volume of the E-4 exhaust system, the length of the duct work had to be estimated using room locations. The isometric drawings listed the diameters of the duct work and the room numbers where the ducts originated. A plan view of the E-4 exhaust system could not be found; therefore, the Tength of the duct work was estimated from the location of the rooms in the PFP. To estimate the overall volume, a nominal duct size was selected after examining the given diameters. The diameter was assumed to be $30.5 \mathrm{~cm}$ (12 in.).

Table 5-14 1ists the estimated volume of E-3 and E-4 exhaust. Using the same theory described for the supply air system, it is assumed that the final values of E-3 and E-4 exhaust are underestimated by 10 percent. After calculating the volumes of duct work for the E3 and E4 systems, it was discovered that the E3 system contributes around 48 percent of the total volume of duct work in 234-5Z, and the E4 system contributes around 8 percent of the total volume. The differences in percentages may be attributed to the relatively small diameter of a large portion of the E4 duct work. The E3 system contains several sections of enormous ducting (e.g., $40 \mathrm{~m}$ [135 ft] of $3.7 \times 3.0 \mathrm{~m}[144 \times 120 \mathrm{in}$.$] duct work).$

The E-3 exhaust system is considered LLW because it directly exhausted rooms that had a potential for contamination. It is assumed that only the E-4 duct work from the gloveboxes and hoods to the first filterbox will be TRU waste. By examining the isometric drawings of the E-4 exhaust system it was determined that the majority of duct work from the gloveboxes to the first filterbox was vertical duct work. Therefore the vertical E-4 duct work is TRU waste. The other duct work in the E-4 exhaust system will be considered LLW. The total amount of LLW from the exhaust ventilation system in 234-5Z is $2081 \mathrm{~m}^{3}$. The total amount of TRU waste is $23 \mathrm{~m}^{3}$ (see Table 5-14).

\subsubsection{Plutonium Reclamation Facility Supply and Exhaust}

The drawings used to determine the total volume of supply and exhaust duct work in 236-Z are listed in Table 5-13. Drawing H-2-29675 shows the general layout of duct work in the PRF, and Drawing $\mathrm{H}-2-29602$ gives the dimensions of the overall PRF Building and the internal rooms. Using both drawings, an estimate was made of all the duct work. The air supply system duct work was separated from the exhaust duct work. A distinction between E-3 and E-4 exhaust was not necessary in determining TRU and LLW. Examining the total supply and exhaust systems, it was estimated that 28 percent of a 11 duct work in 236-Z was exhaust duct work. This appears to be a low percentage when compared to the percentage of exhaust duct work in 234-5Z. However, the majority of the gloveboxes and hoods in PRF are exhausted into the inner chamber. This process uses far less duct work than exhausting the gloveboxes and hoods to 291-Z, as is done in other buildings. In addition, the amount of TRU waste is very low. The duct work connecting the gloveboxes to the first filterbox was considered to be TRU. The volume of supply and exhaust duct work in PRF is listed in Table 5-14. The table also contains the amount of TRU waste in PRF. 


\subsubsection{1-Z Exhaust}

An estimate of the total volume of Building $241-Z$ was made using the dimensions provided in the plant description. It was determined that the volume of $241-Z$ was only 0.7 percent of the volume of 234-5Z (see Table 5-14). Therefore, it was assumed that the duct work in the building would not contribute significantly to the overall total volume of duct work in the PFP complex. This theory is also supported by examining the plan drawing of duct work in 241-Z (see Table 5-13).

\subsubsection{6-ZA Exhaust}

Building 2736-ZA is approximately the same size as $241-Z$. Therefore it was determined that the duct work in this building would not contribute significantly to the overall total volume of duct work in the PFP complex. The drawings that support the hypothesis are listed in Table 5-13.

\subsubsection{6-ZB Supply and Exhaust}

The drawings used to determine the total volume of supply and exhaust duct work in 2736-ZB are listed in Table 5-13. The drawings contained all dimensions needed to determine the volume of the duct work. A distinction between E3 and E4 exhaust was not determined. The air supply and exhaust systems were considered LLW. This was assumed because the system is fairly new and there has been very little chance of backflow through the system. The total volume of LLW is listed in Table 5-14.

\subsubsection{Volume of Ventilation System}

Table 5-14 details the volumetric results for the PFP ventilation systems. The supply system has a volume of $1,774 \mathrm{~m}^{3}, a 17$ of which is LLW. The exhaust system has a volume of $2,165 \mathrm{~m}^{3}$, of which 99 percent is $L L W$ and 1 precent is TRU.

\subsection{MISCELLANEOUS EQUIPMENT}

Table 5-15 provides a 1 ist of miscellaneous D\&D solid waste that includes location by building and room number, dimensions, and volume. The volume of waste does not include equipment that is expected to be removed prior to D\&D. Contaminated items encountered on drawings or during the walkdown that are not included in the other physical categories are included in Table 5-15. The total volume of solid waste expected from miscellaneous equipment is $165 \mathrm{~m}^{3}$. This entire volume has been classified as TRU waste because the contamination levels of the items are unknown. In many cases, these items are listed in Table 5-15 based on statements made by PFP personnel. This table was verified and corrected during the walkdown. Dimensions are from various facility drawings unless specified as a walkdown estimate. In the latter case, dimensions were estimated visually to the nearest three decimeters (foot). 
Table 5-15. Miscellaneous Equipment. (2 sheets)

\begin{tabular}{|c|c|c|c|c|c|c|c|c|}
\hline \multirow[t]{2}{*}{$\begin{array}{l}\text { Building } \\
\text { number }\end{array}$} & \multirow[t]{2}{*}{$\begin{array}{l}\text { Room } \\
\text { number }\end{array}$} & \multirow[t]{2}{*}{ Miscellaneous equipment } & \multicolumn{4}{|c|}{$\begin{array}{l}\text { Dimensions } \\
(\mathrm{m})\end{array}$} & \multirow[t]{2}{*}{$\begin{array}{l}\text { Volyme } \\
\left(m^{3}\right)\end{array}$} & \multirow[t]{2}{*}{$\begin{array}{l}\text { Information } \\
\text { origin }\end{array}$} \\
\hline & & & Length & Depth & Height & Diameter & & \\
\hline \multirow[t]{20}{*}{$234-52$} & 132 & $\begin{array}{l}\text { Mass spectrometer with } 10.2-\mathrm{cm}-(4-\text { in.- }) \\
\text { diameter exhaust }\end{array}$ & 1.83 & 0.91 & 2.44 & $\cdots$ & 4.08 & Halkdown \\
\hline & 227 & Pressure gauges & -- & -- & 2.44 & 0.28 & 0.15 & Drauings \\
\hline & \multirow[t]{6}{*}{ 228-A } & Agitators on top of glovebox (2) & $-\cdot$ & -- & 0.61 & 0.30 & 0.04 & Drawings \\
\hline & & Ratiomotor & 0.15 & 0.23 & 0.36 & -- & 0.01 & Drawings \\
\hline & & Sand hopper & -- & -- & 0.51 & 0.20 & 0.02 & Drawings \\
\hline & & Vacuum assembly & -- & $\cdots$ & 0.38 & 0.05 & 0.001 & Drawings \\
\hline & & Sphincter seal assembly & -- & -- & 0.25 & 0.23 & 0.01 & Drawings \\
\hline & & Turntable drive assembly & -- & $\cdots$ & 0.13 & 0.13 & 0.002 & Drawings \\
\hline & \multirow[t]{2}{*}{$228-B$} & Cutter assembly & $\cdots$ & $-\cdot$ & 0.30 & 0.08 & 0.001 & Drawings \\
\hline & & sweep assembly & -- & -- & 0.30 & 0.28 & 0.02 & Drawings \\
\hline & \multirow[t]{3}{*}{$228-C$} & Drive shaft & $-\cdot$ & -- & 0.20 & 0.10 & 0.002 & Drawings \\
\hline & & Motor & $\therefore$ & $\cdots$ & 0.53 & 0.15 & 0.01 & Dranings \\
\hline & & SHeep actuator assemblies (2) & -- & -- & 1.17 & 0.08 & 0.01 & Drawings \\
\hline & \multirow[t]{2}{*}{$235-8$} & Drive assembly & -- & $\cdots$ & 0.69 & 0.30 & 0.05 & Drauings \\
\hline & & Seal out platform & 0.46 & 0.41 & 0.03 & -- & 0.005 & Drawings \\
\hline & \multirow[t]{2}{*}{$235-E$} & Hydraul ic ram, pump & 1.22 & 1.22 & 1.22 & -- & 1.81 & Nalkdown \\
\hline & & Digester vessels (2) & $\cdots$ & $-\cdot$ & 0.91 & 1.52 & 3.33 & Halkdown \\
\hline & \multirow[t]{3}{*}{320} & Recirculating air-conditioning units (3) & 1.22 & 1.52 & 0.91 & $\cdots$ & 5.10 & Halkdown \\
\hline & & Acme chiller & 1.52 & 0.61 & 0.91 & -- & 0.85 & Wolkdown \\
\hline & & Vacuum filters, $66 \mathrm{~cm}(26$ in.) (4) & $-\cdot$ & $-\cdot$ & 0.61 & 0.91 & 1.60 & Walkdown \\
\hline \multirow[t]{3}{*}{$236-2$} & \multirow[t]{2}{*}{35} & Vacurm pumps numbers 1 and 2 & 0.61 & 0.61 & 0.61 & -- & 0.23 & walkdown \\
\hline & & Compressor tanks VF-1 and VF-2 & $-\cdot$ & $\cdots$ & 0.91 & 0.91 & 0.60 & Halkdown \\
\hline & 43 & Lead chases (2) & 1.07 & 0.15 & 0.15 & -- & 0.02 & Halkdown \\
\hline
\end{tabular}


Table 5-15. Miscellaneous Equipment. (2 sheets)

\begin{tabular}{|c|c|c|c|c|c|c|c|c|}
\hline \multirow[t]{2}{*}{$\begin{array}{l}\text { Building } \\
\text { number }\end{array}$} & \multirow[t]{2}{*}{$\begin{array}{l}\text { Room } \\
\text { number }\end{array}$} & \multirow[t]{2}{*}{ Miscellaneous equipment } & \multicolumn{4}{|c|}{$\begin{array}{c}\text { Dimensions } \\
\text { (m) }\end{array}$} & \multirow{2}{*}{$\begin{array}{l}\text { Volume } \\
\left(m^{2}\right)\end{array}$} & \multirow{2}{*}{$\begin{array}{l}\text { Information } \\
\text { origin }\end{array}$} \\
\hline & & & Length & Depth & Height & Diameter & & \\
\hline \multirow[t]{2}{*}{$241-2$} & \multirow[t]{2}{*}{ Building } & Exhaust fan EF-22-25D & $\cdots$ & $\cdots$ & 0.30 & 0.91 & 0.20 & Halkdown \\
\hline & & Exhaust fan EF-21-25D & $\cdots$ & $\cdots$ & 0.20 & 0.61 & 0.06 & Halkdown \\
\hline \multirow[t]{6}{*}{$291-2$} & \multirow[t]{4}{*}{501} & Air compressors (2 new) & 1.22 & 1.52 & 1.83 & - & 3.40 & Halkdown \\
\hline & & Air compressors $(2$ old $)$ & 1.83 & 0.61 & 0.91 & $\cdots$ & 1.02 & Halkdown \\
\hline & & Air compressors $\left(621 \mathrm{kPa}\left[90\left(\mathrm{~b} / \mathrm{in}^{2}\right]\right)\right.$ & 1.22 & 2.44 & 1.52 & -- & 4.53 & Halkdown \\
\hline & & Vacuum pumps, $43 \mathrm{~cm}$ (17 in.) (2) & $\cdots$ & $\cdots$ & 0.91 & 0.6096 & 0.27 & Halkdown \\
\hline & 502 & Fans EF5-EF7, and EF8 & 3.05 & 2.74 & 1.83 & $\cdots$ & 61.16 & Halkdown \\
\hline & 509 & Fans EF1-EF4 and EF9 & 3.05 & 2.74 & 1.83 & - & 76.46 & Walkdown \\
\hline & & & & & \multicolumn{2}{|c|}{ Total volume } & 165.04 & \\
\hline
\end{tabular}




\subsection{SUHMARY}

In this section, the results presented in Section 5.0 by natural physical category are summarized by solid waste category. These results were derived from a document search, including PFP engineering drawings, and a walkdown. Research included interviews with key PFP personnel (see Acknowledgements).

Because of the uncertainties presented in Section 4.1 , several global assumptions were made (see Section 4.2). The reader must understand these assumptions when applying or interpreting the results. Additional assumptions made during the project are noted where they apply.

The accuracy of this report is limited to that of the available documentation. While the walkdown provided verification of the existence of the items included in this report, there are certainly items which were not included.

Table 6-1 and Figures 6-1 and 6-2 present the results of this project. Volumes are given in cubic meters. A total volume of $5,489 \mathrm{~m}^{3}$ is expected in solid waste. This total is expected to be 1 percent DSW, 71 percent LLW, 21 percent TRU, and 7 percent TRU MW. Assuming that tanks containing regulated chemicals, or heels, will be removed from the gloveboxes, all of the gloveboxes and hoods have been classified as TRU waste. Mixed wastes are mainly from tanks and piping. The large amount of LLW is mainly due to the ventilation systems $\left(3,913 \mathrm{~m}^{3}\right)$, which are assumed to be uncompacted. A11 miscellaneous equipment that was known to be contaminated was conservatively assumed to be TRU, since the extent of the contamination was unknown. 
Table 6-1. Decontamination and Decommissioning Solid Waste Volume Summary.

\begin{tabular}{|l|c|c|c|c|c|}
\hline \multirow{2}{*}{ Physical category } & \multicolumn{5}{|c|}{ Waste type $\left(\mathrm{m}^{3}\right)$} \\
\cline { 2 - 6 } & DSW & LLW & TRU & TRU MH & Total volume \\
\hline Gloveboxes and hoods & 0 & 0 & 856.17 & 0 & 856.17 \\
\hline Internal tanks & 0.54 & 0 & 0 & 1.18 & 1.73 \\
\hline External tanks & 40.26 & 0 & 0 & 132.94 & 173.20 \\
\hline Piping & 0.40 & 0 & 8.35 & 89.80 & 98.55 \\
\hline $\begin{array}{l}\text { Ventilation } \\
\text { Filterboxes } \\
\text { HEPA filters }\end{array}$ & 0 & $3,913.00$ & 26.00 & 0 & $3,939.00$ \\
\hline Miscellaneous equipment & 0 & 0 & 100.46 & 0 & 100.46 \\
\hline Totals & 0 & 0 & 15.45 & 139.02 & 154.47 \\
\hline
\end{tabular}

DSW = Dangerous solid waste.

HEPA = High-efficiency particulate air (filter).

$L L W=$ Low-level waste.

TRU = Transuranic.

TRU MW = Transuranic mixed waste. 
Figure 6-1. Decontamination and Decommissioning Solid Waste CTassification by Percentage.

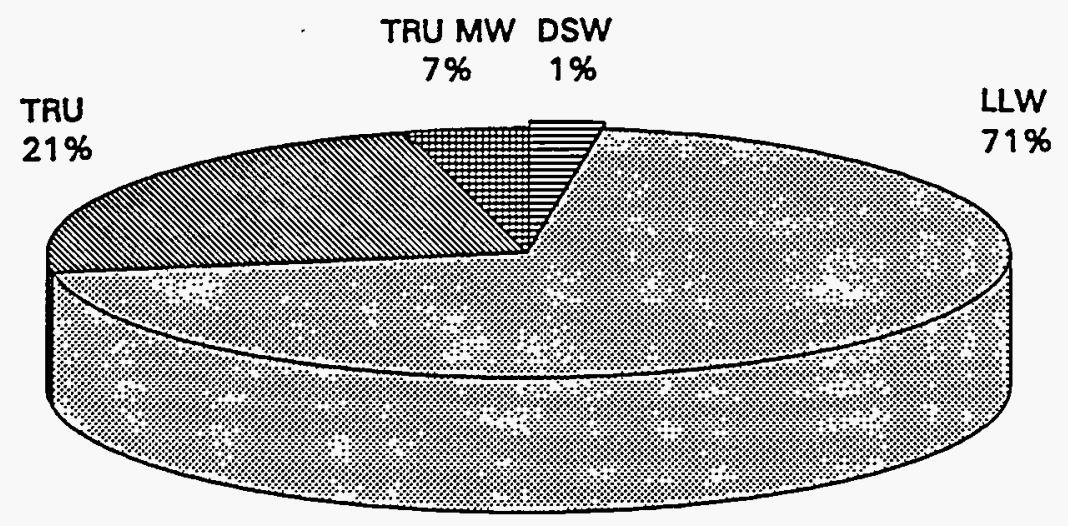

目DSW 圆 LLW $\mathbb{N R U}$ TRU MW


WHC-EP-0829

Figure 6-2. Decontamination and Decommissioning Solid Waste Classification by Volume.

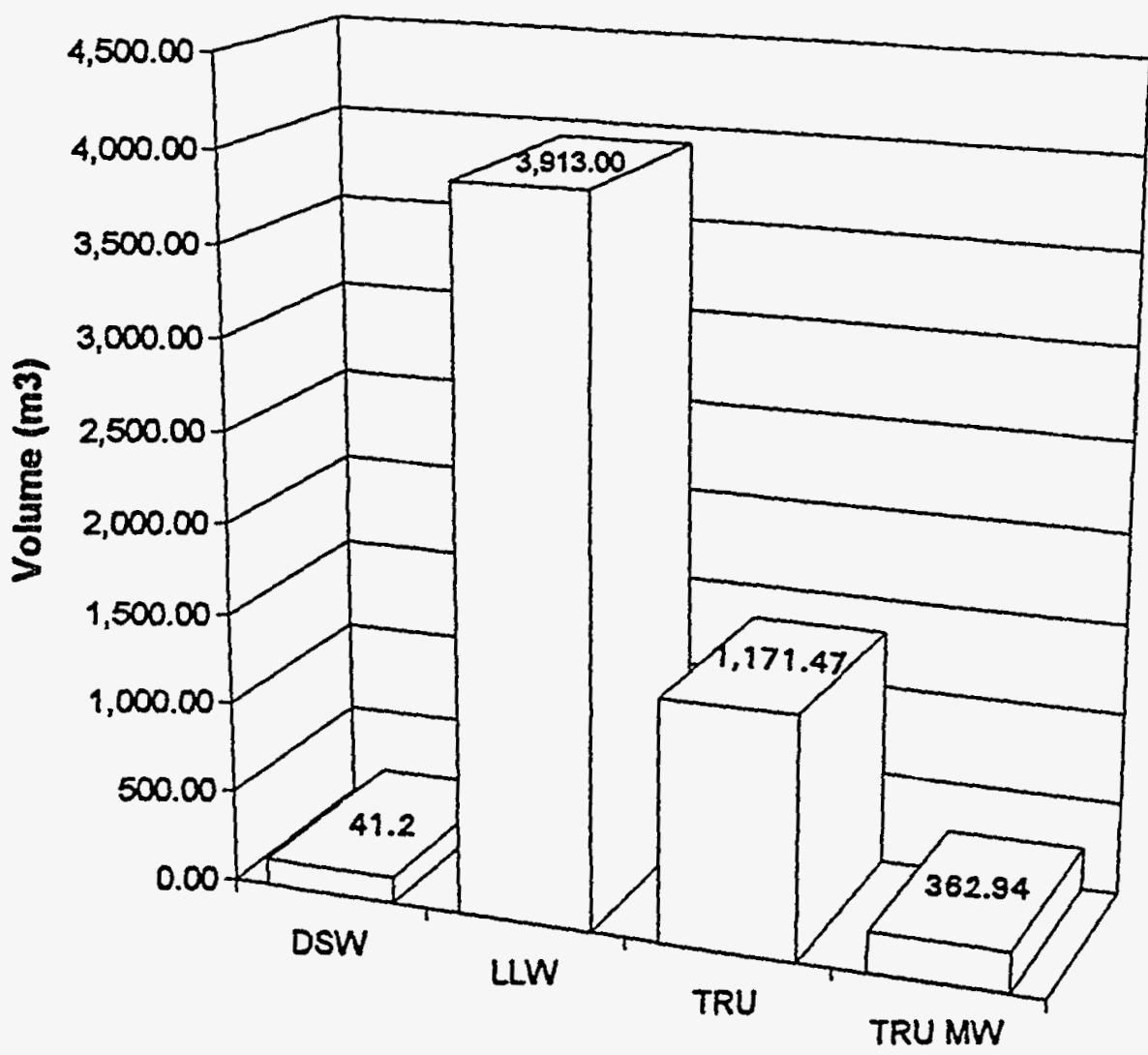


HHC-EP-0829

\subsection{REFERENCES}

\subsection{CODES AND REGULATIONS}

10 CFR 61, 1992, "Licensing Requirements for Land Disposal of Radioactive Waste," Code of Federal Regulations, as amended.

WAC 173-303, 1990, "Dangerous Waste Regulations," Section 040, "Definitions," Washington Administrative Code, as amended.

\subsection{DOCUMENTS}

Ballinger, M. Y., and R. B. Hall, 1989, A History of Hajor Facilities and Processes Involving Radioactive Haterial, PNL 6964, Pacific Northwest Laboratory, Richland, Washington.

Burns, L. E., 1978, "Neutron Monitoring of Waste Tanks for Plutonium and Moisture - Interim Report," Internal letter No. 60130-78-96, August 1, 1978, Rockwell Hanford Operations, Richland, Washington.

Demiter, J. A., D. R. Duncan, W. E. Meeuwen, R. W. Reddinger, and M. S. Zuonar, 1991, Historical Report on the Retrievably Stored Division of Military Application Equipment at the Hanford Site, WHC-IP-0791, Westinghouse Hanford Company, Richland, Washington.

Duncan, R. A., 1985, 242-Z Decontamination and Decommissioning Extension Building: Engineering Study, SD-DD-ES-006, Rockwe11 Hanford Operations, Richland, Washington.

Harlow, D. G., 1975, "361-Z History," Internal letter, October 30, 1975, Atlantic Richfield Hanford Company, Richland, Hashington.

Kasper, R. B., 1982, 216-Z-12 Crib Status Report, RHO-LD-166, Rockwel1 Hanford Operations, Richland, Washington.

Navarro, G. E., 1994, System Design Description: PFP Closed Loop Cooling System, WHC-SD-CP-SDD-015, Rev. 1, Westinghouse Hanford Company, Richland, Washington.

Pottmeyer, J. A., L. C. Amato, D. R. Duncan, J. D. Franklin, R. A. Hyre, R. M. Lowy, and J. S. Millar, 1994, Characterization of Decontamination and Decommissioning Wastes Expected from the Major Processing Facilities in the 200 Areas, WHC-EP-0787, Westinghouse Hanford Company, Richland, Washington.

WHC, 1991, Plutonium Finishing Plant Final Analysis Report, WHC-SD-CP-SAR-021, Westinghouse Hanford Company, Richland, Washington.

WHC, 1993, Plutonium Finishing Plant Standard Practices: Handle and Package Solid Waste in 55-Gallon Drums, 20-170-015, Westinghouse Hanford Company, Richland, Washington. 
WHC, 1994a, Criticality Prevention Specifications [for PFP], CPS-Z-165, Westinghouse Hanford Company, Richland, Washington.

WHC, 1994b, "Plutonium Finishing Plant," Hanford Fact Sheet, RL W94-028, Westinghouse Hanford Company, Richland, Washington.

Will is, N. P., and G. C. Triner, 1993, Hanford Site Radioactive Solid Waste Acceptance Criteria, WHC-EP-0063-4, Hestinghouse Hanford Company, Richland, Washington.

\subsection{DRAWINGS}

H-2-131559, 1994, Testab7e HEPA Fi7ter Schedule, Westinghouse Hanford Company, Richland, Washington.

H-2-16414, 1974, Process Drainage Below First Floor, Atlantic Richfield Hanford Company, Richland, Washington.

H-2-16415, 1987, Process Drainage, Rockwel1 Hanford Operations, Richland, Washington.

H-2-16419, 1993, Waste Disposal Facilities - Waste Sumps and Storage Tank Pit Arrangement, Westinghouse Hanford Company, Richland, Washington.

H-2-16420, 1952, Roof Drainage, General Electric, Richland, Washington.

H-2-16421, 1973, Underground Services, Sewer and Water, Atlantic Richfield Hanford Company, Richland, Washington.

H-2-16425, 1974, Part First Floor P7an Process Drainage, Atlantic Richfield Hanford Company, Richland, Washington.

H-2-16429, 1974, Duct Leve7 Plan Ventilation, Atlantic Richfield Hanford Company, Richland, Washington.

H-2-16432, 1966, Second Floor Plan Ventilation, Douglas United Nuclear, Richland, Washington.

H-2-16435, 1966, Ventilation Sections, Douglas United Nuclear, Richland, Washington.

H-2-16436, 1965, Ventilation Section B-H2-16436, Douglas United Nuclear, Richiand, Washington.

H-2-16437, 1965, Ventilation Sections, Douglas United Nuclear, Richland, Washington.

H-2-16438, 1965, Ventilation Section D-H-2-16438, Douglas United Nuclear, Richi and, Hashington.

H-2-16439, 1976, Ventilation Sections, Atlantic Richfield Hanford Company, Richl and, Washington. 
H-2-16440, 1974, Ventilation Section F-CH-2-16440, Atlantic Richfield Hanford Company, Richland, Washington.

H-2-16441, 1966, Ventilation Sections, Douglas United Nuclear, Richland, Washington.

H-2-16442, 1974, Ventilating Sections, Atlantic Richfield Hanford Company, Richland, Washington.

H-2-16443, 1965, Ventilation Sections, Douglas United Nuclear, Richland, Washington.

H-2-16444, 1965, Ventilation Section and Hanger Details, Douglas United Nuclear, Richland, Washington.

H-2-16445, 1965, Ventilation Sections, Douglas United Nuclear, Richland, Washington.

H-2-16446, 1965, Ventilation Sections T-H2-16430 and S-H2-16430, Douglas United Nuclear, Richland, Washington.

H-2-16447, 1966, Ventilation Sections V-V, Douglas United Nuclear, Richland, Washington.

H-2-16473, 1974, Duct Leve7 Plan Service Piping, Atlantic Richfield Hanford Company, Richland, Washington.

H-2-16474, 1975, Duct Level P7an Service Piping, Atlantic Richfield Hanford Company, Richland, Washington.

H-2-16475, 1967, Duct Leve7 Plan Service Piping, Douglas United Nuclear, Richiand, Washington.

H-2-16476, 1967, Duct Level Plan Service Piping, Douglas United Nuclear, Richland, Washington.

H-2-16477, 1966, Duct Level Plan Service Piping, Douglas United Nuclear, Richland, Washington.

H-2-16478, 1982, Duct Level Plan Service Piping, Rockwell Hanford Operations, Richland, Washington.

H-2-16479, 1968, Duct Level P7an Service Piping, Douglas United Nuclear, Richiand, Washington.

H-2-16480, 1974, Duct Level Plan Service Piping, Atlantic Richfield Hanford Company, Richland, Washington.

H-2-19325, 1993, Process and Service Drains, First Floor Plan, Westinghouse Hanford Company, Richland, Washington.

H-2-23400, 1993, E-4 Exhaust Ventilation IEFD System 25A, Westinghouse Hanford Company, Richland, Hashington. 
H-2-23872, 1970, Plot Plan, Outside Services and Facilities, Atlantic Richfield Hanford Company, Richland, Washington.

H-2-24606, 1993, Air Zone System S-2, Westinghouse Hanford Company, Richland, Washington.

H-2-24607, 1993, Air Zone System S-3, Westinghouse Hanford Company, Richland, Washington.

H-2-24608, 1967, Air Zone System S-3A, Atlantic Richfield Hanford Company, Richland, Washington.

H-2-24609, 1993, Air Zone System S-5, Westinghouse Hanford Company, Richland, Washington.

H-2-24923, 1973, 216-Z-1A Modified Process Waste Disposal Plot Plan, Atlantic Richfield Hanford Company, Richland, Washington.

H-2-26060, 1993, HVAC Air Flow Exhaust System, Building Zone 2 and 3, Westinghouse Hanford Company, Richland, Washington.

H-2-26062, 1993, HVAC Air Flow Exhaust System B, Loading Zone 2 and 3, Westinghouse Hanford Company, Richland, Washington.

H-2-26063, 1993, HVAC Air Flow Exhaust System B, Zone 4, Westinghouse Hanford Company, Richland, Washington.

H-2-26066, 1993, HVAC Air Flow Exhaust System B, Loading Zone 4, Westinghouse Hanford Company, Richland, Washington.

H-2-27969, 1993, HVAC Plans, Sections, and Flow Diagram, Westinghouse Hanford Company, Richland, Washington.

H-2-28014, 1992, Process Engineering Flow Schematic Chemical Preparations System, Westinghouse Hanford Company, Richland, Washington.

H-2-28851, 1993, HVAC P7an, Section, Detai7, Westinghouse Hanford Company, Richland, Washington.

$\mathrm{H}-2-29602$, 1993, Architectural Floor Plan at Elevation $0^{\prime} 0$, "Westinghouse Hanford Company, Richland, Washington.

H-2-29646, 1966, Process and Chemical Piping, Duct Level, Douglas United Nuclear, Richland, Washington.

H-2-29657, 1969, Service and Drainage Piping Plans, First and Second Floors, Atlantic Richfield Hanford Company, Richland, Washington.

H-2-29658, 1991, Service and Drainage Piping Plans, Third and Fourth Floors, Westinghouse Hanford Company, Richland, Washington.

$\mathrm{H}-2-29659,1989$, Service and Drainage, Penthouse Piping Plans and Building Section, Westinghouse Hanford Company, Richland, Washington. 
H-2-29661, 1973, Service Piping, Chemical Preparation Plans, Atlantic Richfield Hanford Company, Richland, Washington.

H-2-29663, 1968, Service Piping Equipment Room Arrangement, Atlantic Richfield Hanford Company, Richland, Washington.

H-2-29674, 1994, PRF HVAC Control System Instrumentation Engineering Flow Diagram, Westinghouse Hanford Company, Richland, Washington.

H-2-29675, 1994, Ventilation Layout, Westinghouse Hanford Company, Richland, Washington.

H-2-29676, 1994, Ventilation, First and Second Floor P7ans, Westinghouse Hanford Company, Richland, Washington.

H-2-29677, 1965, Ventilation, Third and Fourth Floor Plans, Douglas United Nuclear, Richland, Washington.

H-2-32528, 1973, Z Plant Liquid Waste Disposal Sites 216-Z Series, Atlantic Richfield Hanford Company, Richland, Washington.

H-2-43517, 1972, Process Pipe Trench Arrangement, Atlantic Richfield Hanford Company, Richland, Washington.

H-2-44511, 1973, Area Map, 200 West Facilities, Atlantic Richfield Hanford Company, Richland, Washington.

H-2-80168, 1988, HVAC Plan, West Ha7t and Sections, Westinghouse Hanford Company, Richland, Washington.

H-2-80169, 1984, HVAC Plan, East Half and Sections, Rockwell Hanford Operations, Richland, Washington.

H-2-80203, 1984, HVAC Laboratory Ventilation P7ans, Detai7, Rockwell Hanford Operations, Richland, Washington.

H-2-80208, 1984, Laboratory Glove Box, Rockwel1 Hanford Operations, Richland, Hashington.

H-2-93504, 1994, Engineering Flow Diagram RHC Line HC-4 and HC-6, Westinghouse Hanford Company, Richland, Washington.

\subsection{U.S. DEPARTMENT OF ENERGY ORDERS}

DOE Order 5820.2A, Radioactive Waste Management, U.S. Department of Energy, Washington, D.C.

DOE Order 5400.3, Hazardous and Radioactive Mixed Waste Program, U.S. Department of Energy, Hashington, D.C. 
WHC-EP-0829

This page intentionally left blank.

$7-6$ 


\subsection{BIBLIOGRAPHY}

BTack, D. G., and R. G. Geier, 1979, Z Plant Terminal Cleanout Scoping Study, RHO-CD-806, Rockwe11 Hanford Operations, Richland, Washington.

Brand, J. L., 1993, "Plutonium Finishing Plant Crib Responsibility (PRF RR Punchlist Item 0018), "Internal letter No. 15540-93-JLB-113, September 8), Hestinghouse Hanford Company, Richland, Hashington.

Cimoch, R. S., 1994, Process Flowsheet Document Low Level Waste Treatment Facility Plutonium Finishing Plant, PFD-Z-106-00001, Rev. A-0, Westinghouse Hanford Company, Richland, Washington.

Duncan, D. R., 1993, Characterization of Past and Present Solid Waste Streams from the Plutonium Finishing Plant, HHC-EP-0621, Hestinghouse Hanford Company, Richland, Washington.

Durnil, J. F., 1980, Z Plant Terminal Cleanout Criteria, RHO-CD-805, Rockwell Hanford Operations, Richland, Washington.

Harkner, M. R., 1990, MT-5 and HC-60 Safety Assessment, HHC-SD-CP-SAR-012, Westinghouse Hanford Company, Richland, Washington.

Kronva11, C. M., 1988, Plutonium Reclamation Facility Engineering Technica7 Reference, WHC-SD-CP-TI-122, Westinghouse Hanford Company, Richland, Washington.

RHO, 1979, Contaminated Liquid Disposal Sites, RHO-CD-673, Rockwel1 Hanford Operations, Richland, Washington.

RHO, 1979, Transuranic Distribution Beneath a Retired Underground Disposa7 Facility, Hanford Site, RHO-SA-131, Rockwell Hanford Operations, Richland, Washington.

Roemer, J. J., 1985, Hazard Identification and Evaluation, Calcination Operations, Glovebox HA-4OF, Room 169, Building 234-5Z, SD-CP-SAR-011, Rockwell Hanford Operations, Richland, Washington.

Shoemaker, D. C., 1979, Preliminary Study for Decontamination and Decommissioning of Z-Plant Auxiliary Facilities, D0105ER0008, Rockwell Hanford Operations, Richland, Washington.

Stroup, J. L., 1982a, A-Button Line Terminal Cleanout Process Control P7an, SD-RE-PCP-003, Rockwell Hanford Operations, Richland, Hashington.

Stroup, J. L., 1982b, Plutonium Finishing Plant Terminal Cleanout Criteria, SD-RE-OCD-003, Rockwell Hanford Operations, Richland, Washington.

Stroup, J. L., 1983, Plan of Approach to Selected Decontamination and Decommissioning of Plutonium Finishing Plant Facilities, SD-DD-PAP-001, Rockwel1 Hanford Operations, Richland, Washington. 


\section{WHC-EP-0829}

Teal, J. A., 1991, Plutonium Finishing Plant Safety Analysis Report, WHC-SD-HS-SAR-007, Westinghouse Hanford Company, Richland, Washington.

Valero, 0. J., 1994, Estimation of PUREX Equipment and Materials that are Candidates for Removal and Waste Processing During PUREX Plant Closure, WHC-IP-0977, Westinghouse Hanford Company, Richland, Washington.

Vogt, E. C., 1983, Z Plant Plutonium Handling Operations Safety Analysis Report, RHO-CD-1244, Rockwe 11 Hanford Operations, Richland, Washington.

Washburn, J. F., 1983, Plutonium Finishing Plant Selected Decontamination and Decommissioning: Process Control Plan, SD-DD-PCP-001, Rockwell Hanford Operations, Richland, Washington. 
WHC-EP-0829

APPENDIX A

PLUTONIUM FINISHING PLANT - POTENTIAL CONTAMINATION LEVELS

A-1 
WHC-EP-0829

This page intentionally left blank.

A-2 


\section{PLUTONIUM FINISHING PLANT - POTENTIAL CONTAMINATION LEVELS}

This appendix contains the spreadsheet (generated in Excel 5.0) that was used to classify the Plutonium Finishing Plant's buildings and rooms by potential contamination levels. This classification was then used to focus the project on the contaminated areas of the facility. 


\section{LEGEND}

\section{Ventilation Area}

$$
\begin{aligned}
& 1=\text { Zone } 1 \text { = Cold clean area. } \\
& 2=\text { Zone } 2 \text { = Radiation area. } \\
& 4=\text { Zone } 4 \text { = Area of potential airborne radioactivity. }
\end{aligned}
$$

\section{Normal Dose Levels (mrem/h)}

Note: The mrem/h values are gamma ( $Q F=1)$ plus neutron exposure rates. Dose rate is associated with the principal work station in the area indicated. General area readings are usually $10 \%$ or less of this value.

\section{Radiation Sources}

Note: The 234-5Z foundation and tunnel plan shows radiation sources in a) 1 five tunnels and the $241-Z$ foundation.

\section{Glovebox Location}

$\mathrm{N} / \mathrm{A}=$ Figure does not apply to these buildings/rooms.

YES $=$ One or more gloveboxes are located in this building/room.

NONE $=$ No gloveboxes are located in this building/room.

\section{Radiation Control Access Areas}

$\mathrm{N} /$ Cont. = Not controlled.

$C C A=$ Contamination control areas.

$C A A=$ Controlled access areas.

"Glovebox is CCA, but room is NOT CAA.

\section{Radiological Posting as of January 1994}

ARA = Airborne radioactivity area .

SCA = Surface contamination area .

RCA = Radiological control area.

$C L E A R=$ Not posted, assumed to be free of contamination.

\section{Contamination Classification}

$$
\begin{aligned}
& H=\text { "Hot" process area. } \\
& C=\text { "Cold" process support area. } \\
& N=\text { Not contaminated. }
\end{aligned}
$$

In general, a "-" means that the data were not available using this figure or table. 
Table A-1. Data From WHC-SD-CP-SAR-021, Rev. 0. (21 sheets)

\begin{tabular}{|c|c|c|c|c|c|c|c|c|c|c|c|c|}
\hline $\begin{array}{l}\text { Building } \\
\text { number }\end{array}$ & $\begin{array}{c}\text { Room } \\
\text { number }\end{array}$ & Description & $\begin{array}{l}\text { Vent. } \\
\text { area }\end{array}$ & $\begin{array}{c}\text { Mormal } \\
\text { dose } \\
\text { levels } \\
\text { (mrem/h) } \\
\end{array}$ & $\begin{array}{l}\text { Radiation } \\
\text { sources }\end{array}$ & $\begin{array}{l}\text { Glovebox } \\
\text { location }\end{array}$ & $\begin{array}{c}\text { Radiation } \\
\text { control } \\
\text { access } \\
\text { ereas }\end{array}$ & $\begin{array}{l}\text { Radiological } \\
\text { posting } \\
\text { as of } \\
\text { January } 1994\end{array}$ & $\begin{array}{l}\text { Cont. } \\
\text { class. }\end{array}$ & $\begin{array}{c}\text { "Hot" } \\
\text { items } \\
\text { present }\end{array}$ & $\begin{array}{c}\text { Reference } \\
\text { CPS-2-165 } \\
-X X X X X\end{array}$ & $\begin{array}{c}\text { Reference } \\
\text { drawings } \\
H-2-x x x x x\end{array}$ \\
\hline \multirow[t]{2}{*}{$232-2$} & Bldg. & Incinerator (layaway status) & 4 & $<2.0$ & YES & $\mathrm{H} / \mathrm{A}$ & H/Cont. & SCA/RCA & H & $x$ & 80175 & $\mathrm{H}-9-1002$ \\
\hline & - & $\begin{array}{l}\text { Storage, laundry (SWPs), used } \\
\text { filters }\end{array}$ & - & - & - & - & - & - & - & - & - & - \\
\hline \multirow[t]{23}{*}{$234-52 x$} & Bldg. & Plutonium conversion facility & 4 & H/A & YES & YES & YES & $N / A$ & H & - & $\begin{array}{l}80250 \\
80012\end{array}$ & $\begin{array}{c}\text { No drugs } \\
\text { listed }\end{array}$ \\
\hline & - & Air supply plenum chamber & 1 & $<0.5$ & NO & H/A & N/Cont. & CLEAR & N & - & - & - \\
\hline & 80 & Storage & 1 & $<0.5$ & NO & N/A & N/Cont. & RCA & C & - & - & - \\
\hline & 81 & Office & 1 & $<0.5$ & NO & H/A & H/Cont. & RCA & C & - & - & - \\
\hline & 82 & Office & 1 & $<0.5$ & NO & $N / A$ & N/Cont. & RCA & C & - & - & - \\
\hline & 83 & Office & 1 & $<0.5$ & NO & N/A & N/Cont. & RCA & c & - & - & - \\
\hline & 84 & Men's rest room & 1 & $<0.5$ & NO & N/A & H/Cont. & RCA & C & - & - & - \\
\hline & 85 & Women's rest room & 1 & $<0.5$ & NO & H/A & H/Cont. & RCA & C & - & - & - \\
\hline & 86 & Office & 1 & $<0.5$ & NO & H/A & H/Cont. & RCA & C & $\cdot$ & - & - \\
\hline & 87 & Office & 1 & $<0.5$ & NO & $N / A$ & H/Cont. & RCA & C & $\cdot$ & $\cdot$ & - \\
\hline & 88 & Office & 1 & $<0.5$ & NO & N/A & N/Cont. & RCA & C & $\cdot$ & - & - \\
\hline & 89 & Office & 1 & $<0.5$ & NO & N/A & N/Cont. & RCA & C & - & - & - \\
\hline & 90 & Office & 1 & $<0.5$ & NO & N/A & H/Cont. & RCA & c & - & - & - \\
\hline & 92 & Pipe chase & 1 & $<0.5$ & NO & N/A & N/Cont. & RCA & c & - & - & - \\
\hline & $100-108$ & Office area & 1 & $<0.5$ & NO & N/A & H/Cont. & CLEAR & N & - & - & - \\
\hline & 109 & Air lock & 1 & $<0.5$ & NO & H/A & H/Cont. & CLEAR & N & - & - & - \\
\hline & 110 & Women's locker room & 1 & $<0.5$ & HO & N/A & N/Cont. & CLEAR & N & $\cdot$ & - & - \\
\hline & $110-A$ & Homen's laundry hampers & 1 & $<0.5$ & NO & N/A & N/Cont. & CLEAR & H & - & - & - \\
\hline & $110-8$ & Homen's locker room & 1 & $<0.5$ & HO & N/A & N/Cont. & CLEAR & N & - & $\cdot$ & - \\
\hline & 111 & Men's locker room & 1 & $<0.5$ & No & N/A & H/Cont. & CLEAR & H & - & - & - \\
\hline & 112 & Men's rest room & 1 & $<0.5$ & NO & N/A & H/Cont. & CLEAR & H & - & - & - \\
\hline & 113 & Men's rest room & 1 & $<0.5$ & No & N/A & H/Cont. & CLEAR & N & - & - & - \\
\hline & 114 & - & 1 & $<0.5$ & No & N/A & H/Cont. & CLEAR & N & - & - & - \\
\hline
\end{tabular}




\begin{tabular}{|c|c|c|c|c|c|c|c|c|c|c|c|c|}
\hline- & - & $x$ & $H$ & YDS & $\cdot 24003 / \mathrm{N}$ & $V / N$ & słe & $S \cdot 0>$ & 7 & zJooddns ssajosd & $\Rightarrow 1$ & \\
\hline - & - & $x$ & H & VIS & $\cdot 7405 / N$ & $\forall / M$ & s3i & $0 . \varepsilon>$ & 7 & Ssajojd әرsen & E†1 & \\
\hline - & - & - & H & YJS & $\cdot 7400 / \mathrm{N}$ & $\mathbf{V} / \mathrm{N}$ & s3h & $0.2>$ & 7 & 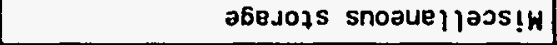 & 276 & \\
\hline - & - & - & H & vวy & $\cdot 740 J / M$ & $V / \mathbb{N}$ & ON & s.0 02 & 7 & 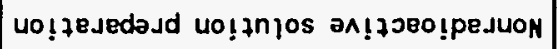 & $|\not|$ & \\
\hline - & - & - & כ & צכy & $\cdot 2405 / N$ & $\forall / \mathbb{N}$ & ON & s'0> & $\varepsilon$ & 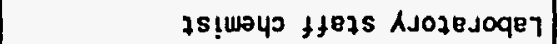 & $3-071$ & \\
\hline - & - & - & כ & עכ & $\cdot$ - ZU0J/N & $V / \mathbb{N}$ & ON & s.0> & $\varepsilon$ & sabeuew Rsozejoge & $8-071$ & \\
\hline - & - & - & 3 & עכy & $\cdot 7405 / N$ & $V / M$ & ON & s.0> & $\varepsilon$ & səo!ndəs jJoddns & $\forall-0 \gg l$ & \\
\hline- & - & $x$ & $H$ & ४⿻8 & - quoj/H & $\forall / N$ & s3i & $* 0^{\circ} 0 \Sigma>$ & 7 & 268s075 & $6 \varepsilon L$ & \\
\hline - & - & $x$ & H & YJS & * *ככว & $\forall / A$ & s3ג & s.0 & $\eta$ & 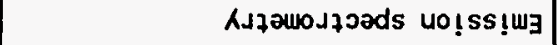 & $\angle \varepsilon l$ & \\
\hline - & - & $x$ & כ & $\forall$ צy & $\cdot 7405 / \mathrm{N}$ & $\forall / N$ & ON & 5.02 & $\varepsilon$ & 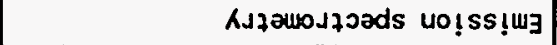 & $9 \varepsilon \downarrow$ & \\
\hline - & - & $x$ & H & YTS & $\cdot \mathcal{Z U O J / N}$ & $\forall / N$ & s3h & 500 & 7 & RJZOUNOJZJ्ads SSEW & SEl & \\
\hline- & - & $x$ & H & YIS & $\cdot 740 J / N$ & $\forall / \mathbb{N}$ & S3A & s.02 & 7 & 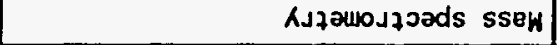 & $7 \varepsilon l$ & \\
\hline- & - & - & H & yכs & $\cdot 7405 / N$ & $\forall / \mathbb{N}$ & ON & s.0> & $\varepsilon$ & wood Xje0 & $\varepsilon \Sigma l$ & \\
\hline- & - & $x$ & 3 & *Jy & $\cdot 7403 / N$ & $\forall / N$ & ON & s.0> & $\varepsilon$ & AJzouroJzozds sseW & $2 \varepsilon 1$ & \\
\hline- & - & - & $H$ & voy & $\cdot 7005 / N$ & $V / N$ & s $3 \lambda$ & 0.22 & 7 & ABsse un!uoznid & $|\Sigma|$ & \\
\hline- & - & - & 3 & vכy & - 2uoj/N & $\forall / N$ & ON & s.0s & $\varepsilon$ & 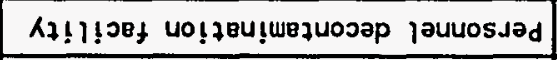 & 921 & \\
\hline- & - & - & N & Bכy & $\cdot 24003 / \mathrm{M}$ & $\forall / N$ & ON & s.0> & 1 & 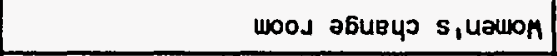 & 721 & \\
\hline- & - & - & N & צษ & $\cdot 7405 / 1$ & $\forall / N$ & ON & s.0> & $\downarrow$ & 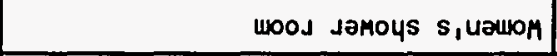 & ह2! & \\
\hline - & - & - & H & yכy & $\cdot 74003 / \mathrm{N}$ & $\forall / N$ & ON & s.0> & $\downarrow$ & -1 & 221 & \\
\hline - & - & - & N & צชะวาכ & $\cdot 74003 / \mathrm{N}$ & $\forall / \mathbb{N}$ & ON & $s \cdot 0>$ & $\downarrow$ & wood zsad S, wawlon & $\lfloor 2\rceil$ & \\
\hline - & - & - & N & צษ כษ & $\cdot 7400 / \mathrm{N}$ & $\forall / N$ & ON & s:0> & $\iota$ & 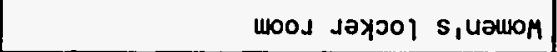 & $02 \downarrow$ & \\
\hline- & - & - & - & - & - & - & - & - & - & - & $\forall \angle L L$ & \\
\hline- & - & - & N & $8 \forall 375$ & $\cdot 74005 / \mathrm{N}$ & $\forall / N$ & ON & s.0> & $\iota$ & 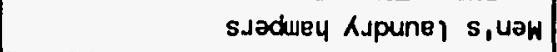 & $\angle I L$ & \\
\hline- & - & - & H & มบฐาว & $\cdot 7400 / \mathrm{N}$ & $V / N$ & ON & s.02 & $\downarrow$ & wood әбиечग $s$, บәW & 911 & \\
\hline- & - & - & N & צชษาכ & $-74003 / \mathrm{N}$ & $\forall / N$ & ON & s.0> & $\downarrow$ & & sll & $\begin{array}{l}(-7400) \\
z 5-7 \varepsilon z\end{array}$ \\
\hline 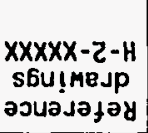 & 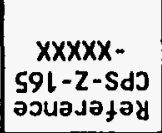 & $\begin{array}{l}\text { zuasajd } \\
\text { surat! } \\
1170 \mathrm{HI1}\end{array}$ & $\begin{array}{l}\cdot \text {-ssepo } \\
\cdot 740 j\end{array}$ & $\begin{array}{c}7661 \text { A.jenuer } \\
\text { to se } \\
\text { 6u! sod } \\
180 ! 6010 ! \mathrm{pey}\end{array}$ & 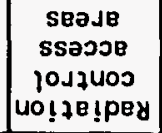 & $\begin{array}{l}\text { uo! } 78501 \\
\text { xоqวл0) }\end{array}$ & $\begin{array}{c}\text { soounos } \\
\text { Uo! } 138 \text { !pey }\end{array}$ & 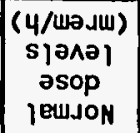 & $\left|\begin{array}{c}80.18 \\
-7401\end{array}\right|$ & 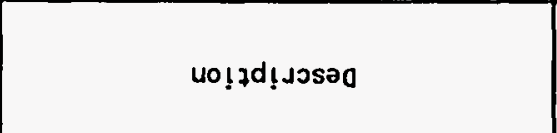 & $\begin{array}{l}\text { Jaqunu } \\
\text { wooy }\end{array}$ & $\begin{array}{l}\text { Jaqunu } \\
\text { Gu!p } 1 ! n g\end{array}$ \\
\hline
\end{tabular}

(sqәәу八 IZ) ${ }^{\circ} 0 \cdot \wedge$ ' 
Table A-1. Data From HHC-SD-CP-SAR-021, Rev. 0. (21 sheets)

\begin{tabular}{|c|c|c|c|c|c|c|c|c|c|c|c|c|}
\hline $\begin{array}{l}\text { Building } \\
\text { number }\end{array}$ & $\begin{array}{l}\text { Room } \\
\text { number }\end{array}$ & Description & $\begin{array}{l}\text { Vent. } \\
\text { area }\end{array}$ & $\begin{array}{c}\text { Normal } \\
\text { dose } \\
\text { levels } \\
\text { (mrem/h) }\end{array}$ & $\begin{array}{l}\text { Radiation } \\
\text { sources }\end{array}$ & $\begin{array}{l}\text { Glovebox } \\
\text { location }\end{array}$ & $\begin{array}{c}\text { Radiation } \\
\text { control } \\
\text { access } \\
\text { areas } \\
\end{array}$ & $\begin{array}{l}\text { Radiological } \\
\text { posting } \\
\text { as of } \\
\text { January } 1994\end{array}$ & $\begin{array}{l}\text { Cont. } \\
\text { class. }\end{array}$ & \begin{tabular}{c|} 
"Hot" \\
items \\
present
\end{tabular} & \begin{tabular}{c|} 
Reference \\
CPS-Z- 165 \\
- XXXXX
\end{tabular} & 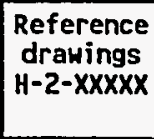 \\
\hline \multirow{24}{*}{$\begin{array}{l}234-52 \\
\text { (cont.) }\end{array}$} & 145 & Physical test & 3 & $<2.0$ & YES & N/A & H/Cont. & $\overline{R C A}$ & $\bar{C}$ & $\bar{x}$ & $\overline{-}$ & $\overline{-}$ \\
\hline & $145-A$ & stock room & 3 & $<0.5$ & NO & N/A & N/Cont. & RCA & C & - & $\overline{7}$ & $\overline{-}$ \\
\hline & 146 & $\begin{array}{l}\text { WIPP repackaging and sample } \\
\text { analysis }\end{array}$ & 4 & $<0.5$ & YES & N/A & N/Cont. & SCA & H & $x$ & - & - \\
\hline & 147 & $\begin{array}{l}\text { Haste packaging and analysis } \\
\text { manager }\end{array}$ & 3 & $<0.5$ & NO & $N / A$ & N/Cont. & SCA & H & - & - & - \\
\hline & 148 & Sample analysis & 4 & $<0.5$ & YES & $N / A$ & N/Cont. & SCA & H & $x$ & - & $\cdot$ \\
\hline & 149 & Sample dissolution & 4 & $<0.5$ & YES & $N / A$ & N/Cont. & SCA & H & $x$ & - & - \\
\hline & 150 & Laboratory shift manager & 3 & $<0.5$ & NO & N/A & N/Cont. & RCA & C & - & - & - \\
\hline & 151 & Miscellaneous counting room & 3 & $<0.5$ & NO & N/A & N/Cont. & RCA & C & - & - & - \\
\hline & 152 & Waste concentrator & 4 & $<2.0$ & YES & $N / A$ & N/Cont. & SCA & H & $x$ & - & - \\
\hline & 153 & Product specification analys is & 4 & $<1.5$ & YES & N/A & N/Cont. & SCA & H & - & - & - \\
\hline & 154 & Product specification analysis & 3 & $<0.5$ & No & N/A & N/Cont. & SCA & H & $x$ & - & - \\
\hline & 155 & Infrared spectrophotometer & 4 & $<0.5$ & YES & N/A & N/Cont. & SCA & H & $x$ & - & - \\
\hline & 156 & Stag and crucible analysis & 4 & $<0.5$ & YES & N/A & N/Cont. & SCA & $H$ & $x$ & - & - \\
\hline & 157 & Product specification analysis & 4 & $<0.5$ & YES & $N / A$ & N/Cont. & SCA & $H$ & $x$ & - & - \\
\hline & 158 & Instrument shop & 3 & $<0.5$ & No & NONE & N/Cont. & RCA & C & - & - & - \\
\hline & $158-A$ & Instrument shop & 3 & $<0.5$ & No & NONE & N/Cont. & RCA & C & - & - & - \\
\hline & 159 & Instrument shop & 3 & $<0.5$ & NO & NONE & N/Cont. & RCA & C & $x$ & - & - \\
\hline & $159-A$ & Instrument shop & 3 & $<0.5$ & NO & NONE & N/Cont. & RCA & C & - & - & - \\
\hline & 161 & RMC shift manager & 3 & $<0.5$ & NO & NONE & N/Cont. & RCA & C & - & - & - \\
\hline & 162 & RHC shift manager & 3 & $<0.5$ & No & NONE & N/Cont. & RCA & c & - & - & - \\
\hline & 163 & Instruments manager & 3 & $<0.5$ & No & NONE & N/Cont. & RCA & c & - & - & - \\
\hline & 164 & RMC shift office & 3 & $<0.5$ & HO & HONE & N/Cont. & RCA & c & - & - & - \\
\hline & 165 & Calcium storage & 3 & $<0.5$ & HO & HONE & N/Cont. & RCA & C & - & - & $\cdot$ \\
\hline & 166 & RMC batch tenks & 4 & $<30.0^{*}$ & YES & YES & CAA & RCA & H & $x$ & 80604 & 25810 \\
\hline
\end{tabular}


Table A-1. Data From WHC-SD-CP-SAR-021, Rev. 0. '(21 sheets)

\begin{tabular}{|c|c|c|c|c|c|c|c|c|c|c|c|c|}
\hline $\begin{array}{l}\text { Building } \\
\text { number }\end{array}$ & $\begin{array}{l}\text { Room } \\
\text { number }\end{array}$ & Description & $\begin{array}{c}\text { Vent. } \\
\text { area }\end{array}$ & $\begin{array}{c}\begin{array}{c}\text { Normal } \\
\text { dose } \\
\text { levels } \\
\text { (mrem/h) }\end{array} \\
\end{array}$ & $\begin{array}{c}\text { Radiation } \\
\text { sources }\end{array}$ & $\begin{array}{l}\text { Glovebox } \\
\text { location }\end{array}$ & \begin{tabular}{|c|} 
Radiation \\
control \\
access \\
areas \\
\end{tabular} & $\begin{array}{l}\text { Radiological } \\
\text { post ing } \\
\text { as of } \\
\text { January } 1994\end{array}$ & $\begin{array}{l}\text { Cont. } \\
\text { class. }\end{array}$ & $\begin{array}{c}\text { "Hot" } \\
\text { items } \\
\text { present }\end{array}$ & $\begin{array}{c}\text { Reference } \\
\text { CPS }-Z-165 \\
-X X X X X\end{array}$ & $\begin{array}{l}\text { Reference } \\
\text { drawings } \\
H-2-X X X X X X\end{array}$ \\
\hline \multirow[t]{25}{*}{$\begin{array}{l}234-52 \\
\text { (cont.) }\end{array}$} & 167 & RMC operations storage & 3 & $<0.5$ & NO & NONE & N/Cont. & $\overline{R C A}$ & C & - & - & - \\
\hline & 168 & RMC ready room/storage & 3 & $<0.5$ & NO & NONE & H/Cont. & RCA & C & - & - & - \\
\hline & 169 & Ash stabilization & 4 & $<2.0$ & YES & YES & N/Cont. & SCA & H & $x$ & 80310 & 93480 \\
\hline & 170 & Ash dissolution & 4 & $<0.5$ & YES & YES & N/Cont. & SCA & H & $x$ & 80320 & 93476 \\
\hline & 171 & Corridor & 3 & $<0.5$ & NO & NONE & N/Cont. & RCA & c & - & - & $\cdot$ \\
\hline & 172 & Maintenance shop (RMC) & 3 & $<2.0$ & NO & NONE & N/Cont. & RCA & C & $\overline{-}$ & - & - \\
\hline & 173 & Maintenance shop & 3 & $<2.0$ & No & NONE & H/Cont. & RCA & c & $\cdot$ & - & - \\
\hline & 174 & SNM storage vault & 3 & $<30.0$ & YES & NONE & CAA & RCA & $H$ & - & - & - \\
\hline & 175 & SNM storage vault & 3 & $<2.0$ & YES & NONE & CAA & RCA & c & - & $\cdot$ & - \\
\hline & 176 & Loading platform & 1 & $<0.5$ & No & H/A & N/Cont. & CLEAR & N & $\cdot$ & - & - \\
\hline & 179 & Process support laboratory & 4 & $<0.5$ & YES & $N / A$ & CAA & SCA & $H$ & $x$ & - & - \\
\hline & 180 & Isotopic research & 3 & $<0.5$ & NO & N/A & N/Cont. & SCA & H & $x$ & $\cdot$ & - \\
\hline & 182 & Process support (microscope) & 3 & $<0.5$ & NO & $N / A$ & N/Cont. & $\mathrm{RCA}$ & C & $\cdot$ & - & - \\
\hline & 183 & Specialized processing & 3 & $<0.5$ & NO & N/A & H/Cont. & RCA & $\mathbf{C}$ & $x$ & - & - \\
\hline & 185 & Miscellaneous storage & 3 & $<0.5$ & NO & N/A & N/Cont. & RCA & C & - & - & $\cdot$ \\
\hline & 186 & storage & 3 & $<0.5$ & No & N/A & N/Cont. & RCA & C & $=$ & $\cdot$ & - \\
\hline & 187 & Open-faced hoods & 3 & $<0.5$ & No & N/A & N/Cont. & SCA & $H$ & $x$ & - & $\cdot$ \\
\hline & 188 & Glovebox/chemical storage & 4 & $<0.5$ & YES & $N / A$ & N/Cont. & RCA & H & $x$ & $\cdot$ & - \\
\hline & 189 & Equipment storage & 3 & $<0.5$ & NO & $N / A$ & N/Cont. & $\mathrm{RCA}$ & C & - & $\cdot$ & - \\
\hline & 190 & Radioactive material storage & 3 & $<30.0^{*}$ & YES & N/A & N/Cont. & RCA & H & - & $\cdot$ & $\cdot$ \\
\hline & 191 & Product support cold laboratory & 3 & $<0.5$ & NO & $H / A$ & N/Cont. & RCA & C & - & - & - \\
\hline & 192 & Orum staging area & 3 & $<0.5$ & No & H/A & N/Cont. & RCA & c & - & - & - \\
\hline & $192-A$ & SNM storage vault & 3 & $<30.0^{*}$ & YES & $N / A$ & CAA & RCA & H & - & - & $=$ \\
\hline & $192-8$ & SNM storage veult & 3 & $>30.0 *$ & YES & $N / A$ & CAA & SCA & H & - & 80340 & $\begin{array}{c}\text { No drwgs } \\
\text { listed }\end{array}$ \\
\hline & $192-C$ & SNM storage vault & 3 & $>30.0^{*}$ & YES & $H / A$ & CAA & SCA & $H$ & - & - & - \\
\hline
\end{tabular}


Table A-1. Data From HHC-SD-CP-SAR-021, Rev. 0. (21 sheets)

\begin{tabular}{|c|c|c|c|c|c|c|c|c|c|c|c|c|}
\hline $\begin{array}{l}\text { Building } \\
\text { number }\end{array}$ & $\begin{array}{c}\text { Room } \\
\text { number }\end{array}$ & Description & $\begin{array}{l}\text { Vent. } \\
\text { area }\end{array}$ & $\begin{array}{c}\text { Hormal } \\
\text { dose } \\
\text { levels } \\
\text { (mrem/h) }\end{array}$ & $\begin{array}{l}\text { Radiation } \\
\text { sources }\end{array}$ & $\begin{array}{l}\text { Glovebox } \\
\text { location }\end{array}$ & \begin{tabular}{|c|} 
Radiation \\
control \\
access \\
areas
\end{tabular} & $\begin{array}{l}\text { Radiological } \\
\text { posting } \\
\text { as of } \\
\text { January } 1994\end{array}$ & $\begin{array}{l}\text { Cont. } \\
\text { class. }\end{array}$ & \begin{tabular}{c|} 
"Hot" \\
items \\
present
\end{tabular} & \begin{tabular}{|c|} 
Reference \\
CPS-Z-165 \\
$-X X X X X$
\end{tabular} & $\begin{array}{c}\text { Reference } \\
\text { drawings } \\
h-2-x x x x x\end{array}$ \\
\hline \multirow{25}{*}{$\begin{array}{l}234-52 \\
\text { (cont.) }\end{array}$} & 192-D & PR can storage & 3 & $<0.5$ & No & N/A & N/Cont. & RCA & C & - & - & $\cdot$ \\
\hline & $192-5$ & Storage & 3 & $<0.5$ & NO & N/A & N/Cont. & $\overline{R C A}$ & $\mathbf{C}$ & $=$ & - & - \\
\hline & $192-N$ & NDA storage & 3 & $<0.5$ & NO & N/A & N/Cont. & RCA & C & - & - & - \\
\hline & 193 & Air lock & 3 & $<0.5$ & NO & N/A & N/Cont. & RCA & C & - & - & - \\
\hline & 194 & Storage vault & 3 & $<0.5$ & NO & N/A & N/Cont. & CLEAR & N & - & - & - \\
\hline & $194-A$ & Material coordinators & 3 & $<0.5$ & NO & N/A & N/Cont. & CLEAR & $\bar{N}$ & - & - & - \\
\hline & 194-B & - & - & - & - & N/A & - & - & N & - & - & - \\
\hline & $195-M$ & - & - & $\cdot$ & - & N/A & - & - & N & - & - & - \\
\hline & $195-\mathrm{H}$ & - & $\cdot$ & - & - & $N / A$ & - & - & H & - & - & - \\
\hline & 196 & Waste drum storage & 3 & $<0.5$ & NO & $N / A$ & N/Cont. & $\mathrm{RCA}$ & c & - & - & - \\
\hline & $196-A$ & Tool crib & $\cdot$ & - & - & $N / A$ & - & - & N & $\cdot$ & - & - \\
\hline & 197 & Waste drum storage & 3 & $<0.5$ & NO & $N / A$ & N/Cont. & RCA & C & - & - & - \\
\hline & $197-A$ & Mixed-waste drum storage & 3 & $<0.5$ & NO & N/A & N/Cont. & RCA & C & - & - & - \\
\hline & $198 \mathrm{~A}$ & Tool crib & 1 & $<0.5$ & NO & $N / A$ & N/Cont. & CLEAR & N & - & - & $\overline{-}$ \\
\hline & 198AL & Air lock & 1 & $<0.5$ & NO & $N / A$ & N/Cont. & CLEAR & H & - & - & - \\
\hline & 199 & Welding shop & 1 & $<0.5$ & NO & $N / A$ & N/Cont. & CLEAR & N & - & - & - \\
\hline & 198 & Maintenance shop & 1 & $<0.5$ & NO & $N / A$ & N/Cont. & CLEAR & N & - & - & - \\
\hline & 200 & Maintenance lockers & 1 & $<0.5$ & NO & N/A & H/Cont. & CLEAR & H & - & - & - \\
\hline & $200-A$ & Maintenance manager & 1 & $<0.5$ & No & N/A & N/Cont. & CLEAR & H & - & $\cdot$ & - \\
\hline & 201 & Pu process vault & 1 & $<0.5$ & NO & N/A & H/Cont. & CLEAR & H & - & $\cdot$ & $\cdot$ \\
\hline & 202 & Pu process support lab & 1 & $<0.5$ & No & $N / A$ & N/Cont. & CLEAR & N & - & - & - \\
\hline & 205 & - & 1 & $<0.5$ & No & $\mathrm{N} / \mathrm{A}$ & H/Cont. & CLEAR & H & - & - & - \\
\hline & 208 & Work authorization system & 1 & $<0.5$ & NO & N/A & N/Cont. & CLEAR & H & - & - & - \\
\hline & $208-A$ & Planner/scheduler & 1 & $<0.5$ & NO & $N / A$ & N/Cont. & CLEAR & H & - & $\cdot$ & - \\
\hline & $208-B$ & Planner/scheduler & 1 & $<0.5$ & NO & N/A & N/Cont. & CLEAR & H & - & - & - \\
\hline
\end{tabular}


Table A-1. Data From WHC-SD-CP-SAR-021, Rev. 0. (21 sheets)

\begin{tabular}{|c|c|c|c|c|c|c|c|c|c|c|c|c|}
\hline $\begin{array}{c}\text { Building } \\
\text { number }\end{array}$ & $\begin{array}{c}\text { Room } \\
\text { number }\end{array}$ & Description & $\begin{array}{l}\text { Vent. } \\
\text { area }\end{array}$ & $\begin{array}{l}\text { Normal } \\
\text { dose } \\
\text { levels } \\
\text { (mrem/h) }\end{array}$ & $\begin{array}{c}\text { Radiation } \\
\text { sources }\end{array}$ & $\begin{array}{l}\text { Glovebox } \\
\text { location }\end{array}$ & $\begin{array}{l}\text { Radiation } \\
\text { control } \\
\text { access } \\
\text { areas }\end{array}$ & \begin{tabular}{|c|} 
Radiological \\
posting \\
as of \\
January 1994
\end{tabular} & $\begin{array}{l}\text { Cont. } \\
\text { class. }\end{array}$ & $\begin{array}{c}\text { "Hot" } \\
\text { items } \\
\text { present }\end{array}$ & \begin{tabular}{|c|} 
Reference \\
CPS-Z-165 \\
-XXXXX
\end{tabular} & $\begin{array}{l}\text { Reference } \\
\text { drawings } \\
H-2-x \times X X X X\end{array}$ \\
\hline \multirow{25}{*}{$\begin{array}{l}234-52 \\
\text { (cont.) }\end{array}$} & 209 & Planner/scheduler & 1 & $<0.5$ & No & N/A & N/Cont. & CLEAR & N & - & $\overline{-}$ & - \\
\hline & $209-A$ & Planner/scheduler & 1 & $<0.5$ & NO & N/A & H/Cont. & CLEAR & N & - & - & - \\
\hline & $209-8$ & Planner/scheduler & 1 & $<0.5$ & NO & N/A & N/Cont. & CLEAR & $\bar{N}$ & - & - & - \\
\hline & 210 & Men's change room hampers & 1 & $<0.5$ & No & $N / A$ & N/Cont. & $\overline{\text { CLEAR }}$ & $\bar{N}$ & - & - & - \\
\hline & 211 & Men's change room & 1 & $<0.5$ & NO & N/A & N/Cont. & CLEAR & $\mathrm{N}$ & - & - & - \\
\hline & 212 & Men's rest room & 1 & $<0.5$ & NO & N/A & N/Cont. & CLEAR & M & - & - & - \\
\hline & $212 \mathrm{~A}$ & - & 1 & $<0.5$ & No & N/A & N/Cont. & CLEAR & $\mathrm{N}$ & - & - & - \\
\hline & 213 & Air locks & 1 & $<0.5$ & NO & N/A & H/Cont. & CLEAR & N & - & - & - \\
\hline & 214 & Men's showers & 1 & $<0.5$ & No & N/A & N/Cont. & CLEAR & N & - & - & - \\
\hline & 215 & Men's rest room & 1 & $<0.5$ & NO & $N / A$ & H/Cont. & CLEAR & N & - & - & - \\
\hline & 216 & Men's change room & 1 & $<0.5$ & NO & N/A & N/Cont. & CLEAR & H & - & - & - \\
\hline & 217 & Analytical lab manager & 1 & $<0.5$ & No & N/A & N/Cont. & CLEAR & N & - & - & - \\
\hline & $217-A$ & Maintenance office & 1 & $<0.5$ & No & N/A & N/Cont. & Clear & H & - & - & - \\
\hline & $218-A$ & Maintenance office & 1 & $<0.5$ & No & N/A & N/Cont. & CLEAR & N & - & - & - \\
\hline & $218-8$ & Maintenance office & 1 & $<0.5$ & No & N/A & N/Cont. & CLEAR & H & - & - & - \\
\hline & $218-\mathrm{c}$ & Maintenance office & 1 & $<0.5$ & No & N/A & N/Cont. & CLEAR & N & - & - & - \\
\hline & 218-D & Maintenance office & 1 & $<0.5$ & No & N/A & N/Cont. & CLEAR & N & - & - & - \\
\hline & $218-E$ & Maintenance office & 1 & $<0.5$ & No & N/A & N/Cont. & CLEAR & N & - & - & - \\
\hline & $218-\mathrm{F}$ & Maintenance office & 1 & $<0.5$ & No & N/A & N/Cont. & CLEAR & N & - & - & - \\
\hline & $221-A$ & Heal th Physics office & 3 & $<0.5$ & No & N/A & N/Cont. & RCA & c & - & - & - \\
\hline & $221-8$ & Sealer repair shop & 3 & $<0.5$ & No & N/A & H/Cont. & RCA & c & - & - & - \\
\hline & $221-\mathrm{c}$ & Radiochemical standards laboratory & 4 & $<2.0$ & YES & N/A & CAA & SCA & H & - & - & - \\
\hline & $221-0$ & Radiochemical standards laboratory & 4 & $<2.0$ & YES & N/A & CAA & SCA & H & $x$ & - & - \\
\hline & $221-E$ & Radiochemical standards laboratory & 3 & $<2.0$ & No & N/A & CAA & SCA & H & $x$ & - & - \\
\hline & $221-\mathrm{F}$ & Heal th Physics office & - & - & - & H/A & - & - & H & - & - & - \\
\hline
\end{tabular}




\begin{tabular}{|c|c|c|c|c|c|c|c|c|c|c|c|c|}
\hline $\begin{array}{c}\text { pazs!! } \\
\text { sEmap oN } \\
\end{array}$ & $0 \varepsilon 008$ & $x$ & H & VIS & $-740 J / N$ & s3i & $s \exists \lambda$ & $* 0^{\circ} 0 \varepsilon>$ & 7 & 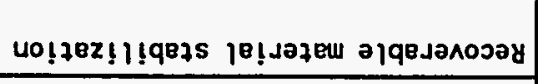 & 8-5£2 & \\
\hline- & 50108 & $x$ & H & yas & WJ & $s 3 \lambda$ & $s \exists \lambda$ & $* 0^{\circ} 0 \Omega>$ & 7 & 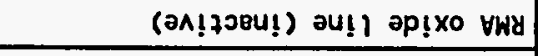 & EV-ธEZ & \\
\hline- & - & $x$ & H & vos & $-7400 / \mathrm{N}$ & saA & S3A & $* 0^{\circ} 0 \Sigma>$ & 7 & 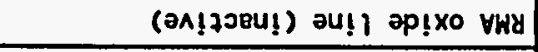 & $2 \forall-\varsigma \S 2$ & \\
\hline- & - & $x$ & H & VOS & $\cdot 740 J / N$ & $\sin$ & $\sin$ & $* 0^{\circ} 0 \Sigma<$ & 7 & 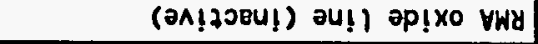 & เV-รฐZ & \\
\hline - & - & - & כ & צכע & $-7400 / \mathrm{N}$ & BNON & ON & $S^{\circ} 0>$ & $\boldsymbol{\varepsilon}$ & †ग0 J!V & \$§2 & \\
\hline- & - & - & 3 & Vכy & $-74005 / \mathrm{N}$ & BNOH & OH & 0.22 & $\varepsilon$ & Sunsp to thaulanseall VaM & $V-y \varepsilon 2$ & \\
\hline- & - & $x$ & 3 & Yכy & $\cdot 340 J / N$ & INOH & $\mathrm{OH}$ & $0.2>$ & $\varepsilon$ & J075eduros ySBJ1 & $7 \varepsilon 2$ & \\
\hline- & - & - & J & Yכy & $\cdot 740 J / N$ & $\forall / N$ & OH & s.0> & $\varepsilon$ & แOOd & 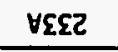 & \\
\hline- & - & - & 3 & Yכy & $-740 J / N$ & BNON & ON & $0.2>$ & $\varepsilon$ & 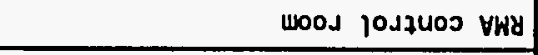 & $\varepsilon \varepsilon 2$ & \\
\hline- & - & $x$ & H & VyV & WVJ/YJJ & $\forall / H$ & ON & s.0> & 7 & soqqnjos $\quad H H$ & VZEZ & \\
\hline 83472 & $\varepsilon 0908$ & $x$ & H & טכy & WUJ/YכJ & $\mathrm{s} \exists \boldsymbol{A}$ & $s \exists \lambda$ & $0.2>$ & 7 & 1100 deqqnuss $\mathrm{HH}$ & टहट & \\
\hline- & - & - & $\mathrm{o}$ & $8 \forall 375$ & - & $\forall / N$ & $\mathrm{OH}$ & $5 \cdot 0>$ & $\varepsilon$ & - & เรZ & \\
\hline 89292 & $090 £ 8$ & $x$ & H & vos & $-740 J / N$ & S3A & $\mathrm{s} 3 \mathrm{~A}$ & $0.2>$ & 7 & 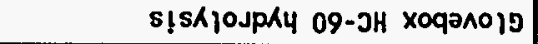 & $3-0 \Sigma 2$ & \\
\hline 69961 & 12908 & $x$ & H & YJS & $-740 J / \mathrm{N}$ & s3a & sal & $* 0^{\circ} 0 \Sigma>$ & 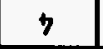 & 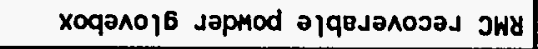 & $8-0 \Sigma 2$ & \\
\hline 20061 & 81908 & $x$ & H & VIS & $\cdot 2400 J / \mathrm{N}$ & S3A & SIA & $* 0^{\circ} 0 \varepsilon>$ & $\rightarrow$ & 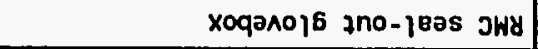 & $\forall$-oø2 & \\
\hline - & - & - & H & \&ะตาว & wo & GNON & ON & $5 \cdot 0>$ & 1 & wood josquos JWy & 622 & \\
\hline เ†26! & 80908 & $x$ & $\mathrm{H}$ & YoS & wo & S3A & s $3 \lambda$ & $* 0^{\circ} 0 \Sigma>$ & 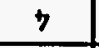 & 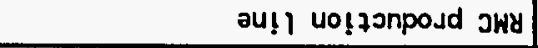 & $0-822$ & \\
\hline L†26ட & 80908 & $x$ & $H$ & YJS & vos & SJA & $\mathrm{SIX}$ & $* 0^{\circ} 0 \Sigma>$ & 7 & au!l Uo!zonposd JWy & 8-822 & \\
\hline$\angle 6602$ & - & - & - & - & - & - & - & - & - & 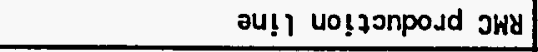 & $\forall-822$ & \\
\hline 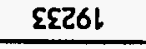 & 01908 & $x$ & $\mathrm{H}$ & YDS & WO & s3A & $S 3 \lambda$ & $* 0^{\circ} 0 \varepsilon>$ & 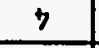 & 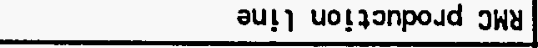 & $\forall-822$ & \\
\hline - & - & $x$ & H & VIS & wo & SaA & s引A & $0.2>$ & 7 & 7no-peo//u! -peol zonposdd & $\angle 22$ & \\
\hline- & - & - & H & עכע & wo & INON & SaA & $* 0^{\circ} 0 \Sigma<<$ & $\varepsilon$ & z!nen Gu! Ipuey zonposd & \$22 & \\
\hline- & - & - & N & \&ษכาכ & - $7400 \mathrm{~J} / \mathrm{N}$ & בNON & ON & $s=0$ & $\underline{\varepsilon}$ & JOP! JJOJ & 722 & \\
\hline- & - & - & N & $=$ & - & $\mathbf{V} / \mathbf{N}$ & - & - & - & $00 !+40$ SD!SAYd प7]ЕәH & $0-122$ & $25-722$ \\
\hline 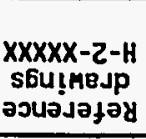 & 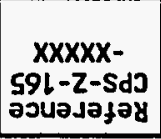 & $\begin{array}{l}\text { zuasajd } \\
\text { swa7! } \\
1170 \mathrm{Hn}\end{array}$ & $\begin{array}{l}\text {-ssep } \\
\cdot 7402\end{array}$ & 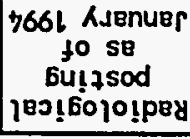 & 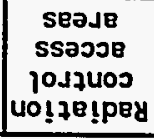 & $\begin{array}{l}\text { uo! } 78501 \\
\text { XoqอNo| }\end{array}$ & $\begin{array}{c}\text { sorJños } \\
\text { uo! } 18 \text { !pey }\end{array}$ & 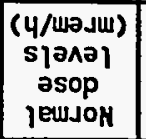 & 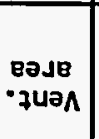 & Uo!?d! IJsรOO & $\begin{array}{l}\text { Jaquinu } \\
\text { wooy }\end{array}$ & $\begin{array}{l}\text { Jaqunu } \\
\text { Bu!pl!ng }\end{array}$ \\
\hline
\end{tabular}


Table A-1. Data From WHC-SD-CP-SAR-021, Rev. 0. (21 sheets)

\begin{tabular}{|c|c|c|c|c|c|c|c|c|c|c|c|c|}
\hline $\begin{array}{l}\text { Building } \\
\text { number }\end{array}$ & $\begin{array}{c}\text { Room } \\
\text { number }\end{array}$ & Description & $\begin{array}{l}\text { Vent. } \\
\text { area }\end{array}$ & $\begin{array}{c}\text { Normal } \\
\text { dose } \\
\text { levels } \\
\text { (mrem/h) }\end{array}$ & $\begin{array}{c}\text { Radiation } \\
\text { sources }\end{array}$ & $\begin{array}{l}\text { Glovebox } \\
\text { location }\end{array}$ & \begin{tabular}{|c|} 
Radiation \\
control \\
access \\
areas
\end{tabular} & \begin{tabular}{|l|} 
Radiological \\
posting \\
as of \\
January 1994
\end{tabular} & $\begin{array}{l}\text { Cont. } \\
\text { class. }\end{array}$ & \begin{tabular}{|c|} 
"Hot" \\
items \\
present
\end{tabular} & \begin{tabular}{|c|} 
Reference \\
CPS-Z-165 \\
$-X X X X X$
\end{tabular} & $\begin{array}{c}\text { Reference } \\
\text { drawings } \\
H-2-x x x x x\end{array}$ \\
\hline \multirow[t]{25}{*}{$\begin{array}{l}234-52 \\
\text { (cont.) }\end{array}$} & $235-c$ & Haste repackaging & 4 & $<1.0$ & YES & YES & H/Cont. & SCA & H & $\overline{-}$ & 80290 & \begin{tabular}{|c|} 
No drwgs \\
listed
\end{tabular} \\
\hline & $235-D$ & Waste drum storage/RADTU & 3 & $<2.0$ & No & H/A & N/Cont. & RCA & H & $\bar{x}$ & - & - \\
\hline & $235-E$ & RADTU filter room & 3 & $<2.0$ & $\cdot$ & N/A & - & - & H & $x$ & - & - \\
\hline & 236 & PR can storage vault & 3 & $<30.0^{\star}$ & YES & N/A & CAA & $\mathrm{RCA}$ & H & $\cdot$ & - & - \\
\hline & $236 \mathrm{~A}$ & - & 3 & $<0.5$ & No & N/A & - & CLEAR & C & - & - & - \\
\hline & 2368 & - & 3 & $<0.5$ & No & N/A & - & CLEAR & c & - & - & - \\
\hline & $236 \mathrm{C}$ & - & 3 & $<0.5$ & No & N/A & - & CLEAR & c & - & - & - \\
\hline & 237 & - & 3 & $<0.5$ & No & H/A & - & CLEAR & C & - & - & - \\
\hline & 238 & - & 3 & $<0.5$ & NO & N/A & - & CLEAR & C & - & - & - \\
\hline & 239 & - & 3 & $<0.5$ & No & H/A & - & CLEAR & c & - & - & - \\
\hline & 240 & - & 3 & $<0.5$ & No & N/A & - & CLEAR & C & - & - & $\bar{F}$ \\
\hline & 241 & - & 3 & $<0.5$ & No & N/A & - & CLEAR & c & - & - & - \\
\hline & 242 & Ready room shipping/receiving & 3 & $<0.5$ & NO & N/A & - & RCA & C & - & - & - \\
\hline & $242 \mathrm{~A}$ & Shift manager product handling & 3 & $<0.5$ & No & N/A & - & RCA & C & - & - & - \\
\hline & 2428 & Shift supervisor product handling & 3 & $<0.5$ & No & N/A & $\cdot$ & RCA & C & $\cdot$ & - & - \\
\hline & 245 & Corridor & 3 & $<0.5$ & No & NONE & N/Cont. & RCA & C & - & $\cdot$ & $\cdot$ \\
\hline & 247 & - & 1 & $<0.5$ & NO & H/A & - & SCA & c & - & - & - \\
\hline & 248 & - & 1 & $<0.5$ & No & N/A & - & RCA & C & - & - & - \\
\hline & 249 & - & 1 & $<0.5$ & No & N/A & - & RCA & c & - & - & - \\
\hline & Mezz & Mezzanine columns $12-22$ & 3 & $<0.5$ & MO & $H / A$ & - & CLEAR & c & - & - & - \\
\hline & 250 & office/storage & 1 & $<0.5$ & NO & H/A & N/Cont. & CLEAR & N & $\overline{-}$ & - & $\overline{-}$ \\
\hline & $251 \mathrm{~A}$ & Office/storage & 1 & $<0.5$ & No & H/A & N/Cont. & CLEAR & N & - & - & $\cdot$ \\
\hline & 2518 & Office/storage & 1 & $<0.5$ & No & H/A & N/Cont. & CLEAR & $N$ & - & - & - \\
\hline & 252 & Office/storage & 1 & $<0.5$ & NO & H/A & H/Cont. & CLEAR & H & - & - & - \\
\hline & 253 & Office/storage & 1 & $<0.5$ & No & H/A & $\mathrm{H} /$ Cont. & CLEAR & N & - & - & - \\
\hline
\end{tabular}


Table A-1. Data From WHC-SD-CP-SAR-021, Rev. 0. (21 sheets)

\begin{tabular}{|c|c|c|c|c|c|c|c|c|c|c|c|c|}
\hline $\begin{array}{l}\text { Building } \\
\text { number }\end{array}$ & $\begin{array}{c}\text { Room } \\
\text { number }\end{array}$ & Description & $\begin{array}{c}\text { Vent. } \\
\text { area }\end{array}$ & $\begin{array}{c}\begin{array}{c}\text { Mormal } \\
\text { dose } \\
\text { levels } \\
\text { (mrem/h) }\end{array} \\
\end{array}$ & $\begin{array}{c}\text { Radiation } \\
\text { sources }\end{array}$ & $\begin{array}{l}\text { Glovebox } \\
\text { location }\end{array}$ & $\begin{array}{c}\text { Radiation } \\
\text { control } \\
\text { access } \\
\text { areas }\end{array}$ & \begin{tabular}{|c|} 
Radiological \\
posting \\
as of \\
January 1994
\end{tabular} & $\begin{array}{c}\text { Cont. } \\
\text { class. }\end{array}$ & \begin{tabular}{|c|} 
"Hot" \\
items \\
present
\end{tabular} & \begin{tabular}{c|} 
Reference \\
CPS-Z-165 \\
$-X X X X X$ \\
\end{tabular} & $\begin{array}{c}\text { Reference } \\
\text { drawings } \\
H-2-X X X X X\end{array}$ \\
\hline \multirow[t]{25}{*}{$\begin{array}{c}234-52 \\
\text { (cont.) }\end{array}$} & 254 & Office/storage & 1 & $<0.5$ & NO & N/A & H/Cont. & $\begin{array}{l}\text { CLEAR } \\
\end{array}$ & N & - & - & - \\
\hline & 255 & Janitors' room & 1 & $<0.5$ & NO & N/A & N/Cont. & $\overline{\text { CLEAR }}$ & $\bar{N}$ & - & - & - \\
\hline & 260 & Duct level & 1 & $<0.5$ & NO & N/A & - & CLEAR & $\bar{N}$ & - & - & - \\
\hline & $260 \mathrm{~A}$ & Air lock & 1 & $<0.5$ & NO & N/A & - & $\overline{\text { CLEAR }}$ & $\bar{N}$ & - & - & - \\
\hline & 261 & Storage & 1 & $<0.5$ & NO & N/A & - & $\begin{array}{l}\text { CLEAR } \\
\end{array}$ & $N$ & - & - & - \\
\hline & 262 & Duct level & 3 & $<2.0$ & YES & N/A & N/Cont. & SCA & $\mathrm{H}$ & $\bar{x}$ & - & - \\
\hline & 263 & Duct level & 3 & $<2.0$ & YES & N/A & N/Cont. & SCA & $\mathrm{H}$ & $\bar{x}$ & 80080 & $\begin{array}{c}\text { No drwgs } \\
\text { listed }\end{array}$ \\
\hline & 264 & Duct level & 3 & $<2.0$ & YES & N/A & H/Cont. & SCA & H & $x$ & - & - \\
\hline & 265 & Duct level & 1 & $<0.5$ & NO & $N / A$ & - & CLEAR & H & - & - & - \\
\hline & 266 & Electrical room & 1 & $<0.5$ & NO & N/A & - & CLEAR & $\mathrm{N}$ & - & - & - \\
\hline & 267 & Telephone swtg room & 1 & $<0.5$ & NO & $N / A$ & $\cdot$ & CLEAR & N & - & - & - \\
\hline & 268 & Storage room & 1 & $<0.5$ & No & $N / A$ & - & CLEAR & N & - & - & - \\
\hline & 269 & Battery storage & 1 & $<0.5$ & NO & N/A & $\overline{-}$ & CLEAR & N & - & - & $\cdot$ \\
\hline & 270 & Duct level & 1 & $<0.5$ & NO & $N / A$ & - & CLEAR & $\bar{c}$ & $\bar{x}$ & - & - \\
\hline & $270 \mathrm{~A}$ & Air lock & 3 & $<0.5$ & NO & $N / A$ & - & CLEAR & H & - & - & - \\
\hline & 271 & - & 3 & $<0.5$ & NO & N/A & $\overline{-}$ & CLEAR & $\bar{c}$ & - & - & $\cdot$ \\
\hline & 272 & Duct level & 1 & $<0.5$ & NO & N/A & - & CLEAR & C & - & - & - \\
\hline & 300 & Foyer & 1 & $<0.5$ & NO & H/A & - & CLEAR & $\mathrm{H}$ & - & - & - \\
\hline & 301 & Maintenance engineers & 1 & $<0.5$ & No & $\mathrm{H} / \mathrm{A}$ & $\mathrm{H} / \mathrm{A}$ & CLEAR & $N$ & - & - & - \\
\hline & $301-A$ & Process engineers & 1 & $<0.5$ & NO & $\mathrm{N} / \mathrm{A}$ & $N / A$ & CLEAR & N & $\cdot$ & - & - \\
\hline & $301-B$ & Staff assistant & 1 & $<0.5$ & NO & $N / A$ & $N / A$ & CLEAR & $\bar{N}$ & - & - & $\cdot$ \\
\hline & $301-C$ & Process engineer & 1 & $<0.5$ & MO & H/A & H/A & CLEAR & $\mathrm{H}$ & - & - & - \\
\hline & $301-0$ & - & 1 & $<0.5$ & NO & N/A & N/Cont. & CLEAR & $\mathrm{H}$ & - & - & $\cdot$ \\
\hline & 302 & Chemical storage & 1 & $<0.5$ & NO & N/A & $\mathrm{N} / \mathrm{A}$ & CLEAR & H & - & - & - \\
\hline & 303 & Process engineer & 1 & $<0.5$ & No & N/A & N/A & CLEAR & H & - & $\overline{-}$ & - \\
\hline
\end{tabular}


Table A-1. Data From WHC-SD-CP-SAR-021, Rev. 0. (21 sheets)

\begin{tabular}{|c|c|c|c|c|c|c|c|c|c|c|c|c|}
\hline $\begin{array}{l}\text { Building } \\
\text { number }\end{array}$ & $\begin{array}{c}\text { Room } \\
\text { number }\end{array}$ & Description & $\begin{array}{l}\text { Vent. } \\
\text { area }\end{array}$ & $\begin{array}{c}\text { Normal } \\
\text { dose } \\
\text { levels } \\
\text { (mrem/h) }\end{array}$ & $\begin{array}{l}\text { Radiation } \\
\text { sources }\end{array}$ & $\begin{array}{l}\text { Glovebox } \\
\text { location }\end{array}$ & $\begin{array}{c}\text { Radiation } \\
\text { control } \\
\text { access } \\
\text { areas }\end{array}$ & $\begin{array}{l}\text { Radiological } \\
\text { post ing } \\
\text { as of } \\
\text { January } 1994\end{array}$ & $\begin{array}{l}\text { Cont. } \\
\text { class. }\end{array}$ & $\begin{array}{c}\text { "Hot" } \\
\text { items } \\
\text { present }\end{array}$ & $\begin{array}{c}\text { Reference } \\
\text { CPS }-Z-165 \\
-X X X X X\end{array}$ & $\begin{array}{l}\text { Reference } \\
\text { drawings } \\
H-2-X X X X X\end{array}$ \\
\hline \multirow{24}{*}{$\begin{array}{l}234-52 \\
\text { (cont.) }\end{array}$} & $303-A$ & Process engineer & 1 & $<0.5$ & NO & N/A & N/A & CLEAR & N & - & - & - \\
\hline & $303-8$ & Process engineer & 1 & $<0.5$ & NO & N/A & $N / A$ & CLEAR & N & - & - & - \\
\hline & $303-C$ & Process engineer & 1 & $<0.5$ & NO & N/A & $\mathrm{H} / \mathrm{A}$ & CLEAR & H & - & - & - \\
\hline & $303-D$ & Repository & 1 & $<0.5$ & No & H/A & $N / A$ & CLEAR & N & - & - & - \\
\hline & $303-E$ & Process engineer & 1 & $<0.5$ & NO & N/A & N/A & CLEAR & $\bar{N}$ & - & - & - \\
\hline & $303-F$ & Envir. and effluents engr. manager & 1 & $<0.5$ & NO & N/A & N/A & CLEAR & M & - & - & - \\
\hline & $303-6$ & Process engineers & 1 & $<0.5$ & NO & N/A & H/A & CLEAR & N & - & - & - \\
\hline & $303-\mathrm{H}$ & Process engineers & 1 & $<0.5$ & NO & N/A & $\mathrm{N} / \mathrm{A}$ & CLEAR & N & $\cdot$ & $\cdot$ & $\cdot$ \\
\hline & $303-J$ & Process engineers & 1 & $<0.5$ & NO & H/A & $H / A$ & CLEAR & H & - & - & $\cdot$ \\
\hline & $303-k$ & Secretary (engineering) & 1 & $<0.5$ & NO & N/A & $H / A$ & CLEAR & N & - & - & - \\
\hline & $303-L$ & Process support manager & 1 & $<0.5$ & NO & N/A & $N / A$ & CLEAR & H & - & $\cdot$ & - \\
\hline & $303-M$ & Process control manager & 1 & $<0.5$ & NO & $N / A$ & $H / A$ & CLEAR & N & - & - & - \\
\hline & 304 & Plastic shop & 1 & $<0.5$ & NO & $N / A$ & $N / A$ & CLEAR & N & - & - & - \\
\hline & $304-A$ & Store room office & 1 & $<0.5$ & NO & N/A & $H / A$ & CLEAR & N & - & - & - \\
\hline & $304-8$ & Property specialist & 1 & $<0.5$ & No & H/A & $\mathrm{H} / \mathrm{A}$ & CLEAR & H & - & - & $\cdot$ \\
\hline & 305 & Lunch room & 1 & $<0.5$ & NO & $H / A$ & $H / A$ & CLEAR & $N$ & - & - & - \\
\hline & $305 \mathrm{~A}$ & - & 1 & $<0.5$ & NO & $H / A$ & - & CLEAR & H & - & - & - \\
\hline & 306 & Conference room & 1 & $<0.5$ & NO & N/A & $N / A$ & CLEAR & N & - & - & - \\
\hline & $306-A$ & Computer room & 1 & $<0.5$ & No & N/A & H/A & CLEAR & N & - & $\cdot$ & - \\
\hline & 307 & Air lock & 1 & $<0.5$ & No & $N / A$ & $H / A$ & CLEAR & H & - & - & - \\
\hline & 308 & Duct level & 4 & $<0.5$ & YES & N/A & N/Cont. & SCA & H & $x$ & 80800 & $\begin{array}{l}\text { No drwgs } \\
\text { listed }\end{array}$ \\
\hline & 309 & E-4 filter room & 4 & $<0.5$ & No & $\mathrm{N} / \mathrm{A}$ & $N / A$ & SCA & H & $x$ & 80250 & $\begin{array}{c}\text { No drwgs } \\
\text { listed }\end{array}$ \\
\hline & 310 & E-4 filter room & 4 & $<0.5$ & NO & N/A & N/A & SCA & H & $x$ & 80250 & $\begin{array}{l}\text { No drwgs } \\
\text { listed }\end{array}$ \\
\hline & 311 & E-3 filter room & 4 & $<0.5$ & NO & N/A & $\mathrm{H} / \mathrm{A}$ & SCA & H & $x$ & 80250 & $\begin{array}{l}\text { No drwgs } \\
\text { listed }\end{array}$ \\
\hline
\end{tabular}


Table A-1. Data From HHC-SD-CP-SAR-021, Rev. 0. (21 sheets)

\begin{tabular}{|c|c|c|c|c|c|c|c|c|c|c|c|c|}
\hline $\begin{array}{l}\text { Building } \\
\text { number }\end{array}$ & $\begin{array}{c}\text { Room } \\
\text { number }\end{array}$ & Description & $\begin{array}{c}\text { Vent. } \\
\text { area }\end{array}$ & 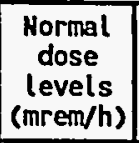 & $\begin{array}{c}\text { Radiation } \\
\text { sources }\end{array}$ & $\begin{array}{l}\text { Glovebox } \\
\text { location }\end{array}$ & \begin{tabular}{|c|} 
Radiation \\
control \\
access \\
areas \\
\end{tabular} & \begin{tabular}{|c|} 
Radiological \\
posting \\
os of \\
January 1994 \\
\end{tabular} & $\begin{array}{l}\text { Cont. } \\
\text { class. }\end{array}$ & \begin{tabular}{|c|} 
"Hot" \\
items \\
present
\end{tabular} & \begin{tabular}{|c|} 
Reference \\
CPS- $z-165$ \\
$-X X X X X$
\end{tabular} & $\begin{array}{l}\text { Reference } \\
\text { drawings } \\
H-2-X X X X X\end{array}$ \\
\hline \multirow[t]{23}{*}{$\begin{array}{l}234-52 \\
\text { (cont.) }\end{array}$} & 312 & E-3 filter room & 4 & $<0.5$ & NO & H/A & N/A & SCA & H & $x$ & 80250 & $\begin{array}{c}\text { No drwgs } \\
\text { listed }\end{array}$ \\
\hline & 313 & E-3 filter room & 4 & $<0.5$ & No & H/A & H/A & SCA & $H$ & $x$ & 80250 & $\begin{array}{c}\begin{array}{c}\text { No drugs } \\
\text { listed }\end{array} \\
\end{array}$ \\
\hline & 314 & E-3 filter room & 4 & $<0.5$ & HO & H/A & N/A & SCA & H & $x$ & 80250 & $\begin{array}{c}\text { Mo drwgs } \\
\text { listed }\end{array}$ \\
\hline & 315 & E-3 filter room & 4 & $<0.5$ & No & H/A & N/A & SCA & $\mathrm{H}$ & $x$ & 80250 & \begin{tabular}{|c|} 
No drugs \\
listed
\end{tabular} \\
\hline & 316 & E-3 filter room & 4 & $<0.5$ & NO & H/A & N/A & SCA & H & $x$ & 80250 & $\begin{array}{c}\begin{array}{c}\text { No drugs } \\
\text { listed }\end{array} \\
\end{array}$ \\
\hline & 318 & E-3 filter room & 4 & $<0.5$ & No & $H / A$ & N/A & SCA & $\mathrm{H}$ & $x$ & 80250 & \begin{tabular}{|c|} 
No drwgs \\
listed
\end{tabular} \\
\hline & 319 & Supply fans & 1 & $<0.5$ & No & N/A & H/A & CLEAR & C & - & - & - \\
\hline & 320 & Filterboxes & 4 & $<2.0$ & YES & N/A & H/A & SCA & $H$ & $\bar{x}$ & 80250 & $\begin{array}{c}\text { No drwgs } \\
\text { listed }\end{array}$ \\
\hline & 321 & Steam supply & 1 & $<0.5$ & no & H/A & $H / A$ & CLEAR & H & $\cdot$ & - & - \\
\hline & $321-A$ & Power control room & 3 & $<0.5$ & No & H/A & $\mathrm{N} / \mathrm{A}$ & CLEAR & C & - & - & - \\
\hline & $321-8$ & Power manager & 1 & $<0.5$ & NO & N/A & $N / A$ & CLEAR & N & $\cdot$ & - & $\cdot$ \\
\hline & $321-c$ & Change room & 1 & $<0.5$ & No & N/A & - & CLEAR & $\mathrm{N}$ & - & - & - \\
\hline & 322 & Electrical shop & 1 & $<0.5$ & NO & H/A & H/A & CLEAR & $\mathrm{N}$ & - & - & - \\
\hline & 323 & Electrical manager & 1 & $<0.5$ & HO & N/A & N/A & CLEAR & $N$ & - & - & - \\
\hline & 324 & Janitors' closet & 1 & $<0.5$ & HO & N/A & $\mathrm{H} / \mathrm{A}$ & CLEAR & $\mathrm{H}$ & - & - & - \\
\hline & 325 & Process engineer & 1 & $<0.5$ & HO & H/A & H/A & CLEAR & N & - & - & $\cdot$ \\
\hline & 326 & Air lock passageway & 1 & $<0.5$ & NO & H/A & N/A & CLEAR & N & - & - & - \\
\hline & 327 & Hamper room & 1 & $<0.5$ & No & N/A & N/A & CLEAR & H & - & - & $\cdot$ \\
\hline & 328 & Men's change room & 1 & $<0.5$ & No & N/A & H/A & CLEAR & N & $\cdot$ & $\cdot$ & $\cdot$ \\
\hline & 329 & Men's toilet & 1 & $<0.5$ & NO & H/A & H/A & CLEAR & N & - & - & - \\
\hline & 330 & Men's shower & 1 & $<0.5$ & NO & H/A & N/A & CLEAR & H & - & - & $\cdot$ \\
\hline & 331 & Men's locker room & 1 & $<0.5$ & No & N/A & N/A & CLEAR & N & - & - & $\cdot$ \\
\hline & 332 & Men's locker room entry & 1 & $<0.5$ & HO & H/A & H/Cont. & CLEAR & H & - & - & - \\
\hline
\end{tabular}


Table A-1. Data From WHC-SD-CP-SAR-021, Rev. 0. (21 sheets)

\begin{tabular}{|c|c|c|c|c|c|c|c|c|c|c|c|c|}
\hline $\begin{array}{l}\text { Building } \\
\text { number }\end{array}$ & $\begin{array}{c}\text { Room } \\
\text { number }\end{array}$ & Description & $\begin{array}{l}\text { Vent. } \\
\text { area }\end{array}$ & $\begin{array}{c}\text { Normal } \\
\text { dose } \\
\text { levels } \\
\text { (mrem/h) } \\
\end{array}$ & $\begin{array}{c}\text { Radiation } \\
\text { sources }\end{array}$ & $\begin{array}{l}\text { Glovebox } \\
\text { location }\end{array}$ & $\begin{array}{c}\text { Radiation } \\
\text { control } \\
\text { access } \\
\text { areas }\end{array}$ & \begin{tabular}{|c|} 
Radiol ogical \\
post ing \\
as of \\
January 1994 \\
\end{tabular} & $\begin{array}{l}\text { Cont. } \\
\text { class. }\end{array}$ & $\mid \begin{array}{c}\text { "Hot" } \\
\text { items } \\
\text { present }\end{array}$ & $\begin{array}{l}\text { Reference } \\
\text { CPS--2-165 } \\
\text {-XXXXX }\end{array}$ & $\begin{array}{l}\text { Reference } \\
\text { dranings } \\
H-2-X X X X X\end{array}$ \\
\hline \multirow{25}{*}{$\begin{array}{l}234-52 \\
\text { (cont.) }\end{array}$} & 333 & Men's toilet & 1 & $<0.5$ & HO & N/A & $N / A$ & CLEAR & H & - & - & - \\
\hline & 333-A & Homen's toilet & 1 & $<0.5$ & No & N/A & N/A & CLEAR & N & - & - & - \\
\hline & 334 & Calcium storage & 1 & $<0.5$ & No & N/A & H/A & CLEAR & N & - & - & - \\
\hline & 335 & Calcium storage & 1 & $<0.5$ & No & N/A & $N / A$ & CLEAR & N & - & - & - \\
\hline & 336 & Chemical makeup & 1 & $<0.5$ & No & N/A & H/A & CLEAR & N & - & - & - \\
\hline & 337 & Chemical makeup & 1 & $<0.5$ & No & N/A & N/A & CLEAR & N & - & - & - \\
\hline & 338 & Het chemical storage & 1 & $<0.5$ & No & N/A & N/A & CLEAR & N & - & - & - \\
\hline & 339 & Men's shower & 1 & $<0.5$ & No & N/A & - & CLEAR & N & - & - & - \\
\hline & 340 & Instrument shop & 1 & $<0.5$ & No & N/A & N/A & CLEAR & $N$ & - & - & - \\
\hline & 341 & IRM/SSDM manager & 1 & $<0.5$ & NO & N/A & N/A & CLEAR & N & - & - & - \\
\hline & 342 & Maintenance engineer & 1 & $<0.5$ & NO & N/A & N/A & CLEAR & N & - & - & - \\
\hline & 343 & Maintenance engineer & 1 & $<0.5$ & No & N/A & H/A & CLEAR & N & - & - & - \\
\hline & 344 & Hallway & 1 & $<0.5$ & No & H/A & N/A & CLEAR & H & - & - & - \\
\hline & $344-A$ & Radiation engineer & 1 & $<0.5$ & No & N/A & H/A & CLEAR & N & - & - & - \\
\hline & 345 & Activity engineer & 1 & $<0.5$ & No & H/A & N/A & CLEAR & H & - & - & - \\
\hline & 346 & Administrative specialist & 1 & $<0.5$ & No & H/A & H/A & CLEAR & N & - & - & - \\
\hline & 347 & IRM/SSDM engineer & 1 & $<0.5$ & No & H/A & N/A & CLEAR & $\mathrm{H}$ & - & - & - \\
\hline & 348 & IRM/SSDM engineer & 1 & $<0.5$ & NO & N/A & N/A & CLEAR & N & - & - & - \\
\hline & 349 & - & 1 & $<0.5$ & No & H/A & $\mathrm{H} /$ Cont. & CLEAR & H & - & - & - \\
\hline & 350 & - & 1 & $<0.5$ & No & N/A & N/Cont. & CLEAR & H & - & - & - \\
\hline & 351 & Entrance to computer room & 1 & $<0.5$ & No & N/A & H/A & CLEAR & H & - & - & - \\
\hline & 390 & Air lock & 1 & $<0.5$ & NO & N/A & N/Cont. & CLEAR & N & - & - & - \\
\hline & 682 & Air lock & - & - & - & - & - & RCA & c & - & - & - \\
\hline & 878AL & Air lock & 1 & $<0.5$ & No & N/A & - & CLEAR & N & - & - & - \\
\hline & $879 \mathrm{AL}$ & Air lock & 1 & $<0.5$ & No & N/A & - & CLEAR & N & - & - & - \\
\hline
\end{tabular}


Table A-1. Data From HHC-SD-CP-SAR-021, Rev. 0. (21 sheets)

\begin{tabular}{|c|c|c|c|c|c|c|c|c|c|c|c|c|}
\hline $\begin{array}{l}\text { Building } \\
\text { number }\end{array}$ & $\begin{array}{c}\text { Room } \\
\text { number }\end{array}$ & Description & $\begin{array}{l}\text { Vent. } \\
\text { area }\end{array}$ & $\begin{array}{c}\text { Normal } \\
\text { dose } \\
\text { levels } \\
\text { (mrem/h) } \\
\end{array}$ & $\begin{array}{c}\text { Radiation } \\
\text { sources }\end{array}$ & $\begin{array}{l}\text { Glovebox } \\
\text { location }\end{array}$ & $\begin{array}{c}\text { Radiation } \\
\text { control } \\
\text { access } \\
\text { areas } \\
\end{array}$ & $\begin{array}{c}\text { Radiological } \\
\text { post ing } \\
\text { as of } \\
\text { January } 1994 \\
\end{array}$ & $\begin{array}{l}\text { Cont. } \\
\text { class. }\end{array}$ & $\begin{array}{c}\text { "Hot" } \\
\text { items } \\
\text { present }\end{array}$ & $\begin{array}{c}\text { Reference } \\
\text { CPS-Z-165 } \\
-X X X X X\end{array}$ & $\begin{array}{l}\text { Reference } \\
\text { drawings } \\
H-2-X X X X X\end{array}$ \\
\hline \multirow{25}{*}{$\begin{array}{l}234-52 A \\
\text { (cont.) }\end{array}$} & 701 & Men's change room & - & - & - & - & - & - & H & - & - & - \\
\hline & 702 & - & - & - & - & - & - & - & N & - & - & - \\
\hline & 703 & - & - & - & - & - & - & - & N & - & - & - \\
\hline & 704 & - & - & - & - & - & - & - & N & - & - & - \\
\hline & 705 & - & - & - & - & - & - & - & $N$ & $\cdot$ & - & - \\
\hline & 706 & - & - & - & - & $\cdot$ & - & - & N & $\cdot$ & - & - \\
\hline & 707 & - & - & - & - & - & - & - & $N$ & - & - & - \\
\hline & 708 & Storage & - & - & - & - & $\cdot$ & - & $N$ & $\cdot$ & - & - \\
\hline & 709 & Corridor & - & - & - & - & - & - & N & - & - & $\cdot$ \\
\hline & 710 & Storage & - & - & $\cdot$ & $\cdot$ & - & - & N & - & - & - \\
\hline & 711 & Storage & - & - & - & - & - & $\cdot$ & N & - & - & - \\
\hline & 712 & - & - & - & - & $\cdot$ & - & - & N & - & - & - \\
\hline & 713 & Fire sprinkler riser room & $\cdot$ & - & - & - & - & - & N & - & - & - \\
\hline & 714 & Computer room & - & - & - & - & - & - & N & - & - & - \\
\hline & 715 & HPT WRAM station & - & - & - & - & - & - & H & $\cdot$ & - & - \\
\hline & 716 & RUP posting area & - & - & - & - & - & - & H & - & - & - \\
\hline & 717 & Self survey station & - & - & - & - & 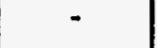 & $\cdot$ & H & - & - & - \\
\hline & 718 & Locker room/lobby & - & - & - & - & $\cdot$ & $\cdot$ & H & - & - & $\cdot$ \\
\hline & 719 & Lobby & $\cdot$ & - & - & - & - & $\cdot$ & H & - & - & $\cdot$ \\
\hline & 720 & RCA entrance/exit thoroughfare & - & - & $\cdot$ & $\cdot$ & - & $\cdot$ & H & - & $\cdot$ & $\cdot$ \\
\hline & 722 & RCA exit security portal & - & $\because$. & - & $\cdot$ & $\cdot$ & - & H & - & - & - \\
\hline & 724 & RCA exit security & $\cdot$ & $\cdot$ & - & $\cdot$ & - & - & H & - & - & - \\
\hline & 726 & RCA entry portal & $\cdot$ & $\cdot$ & - & $\cdot$ & - & - & N & - & - & - \\
\hline & 728 & Security check station & $\cdot$ & - & - & $\cdot$ & - & - & N & - & - & $\cdot$ \\
\hline & 730 & Corridor & - & - & - & - & - & - & H & - & - & - \\
\hline
\end{tabular}




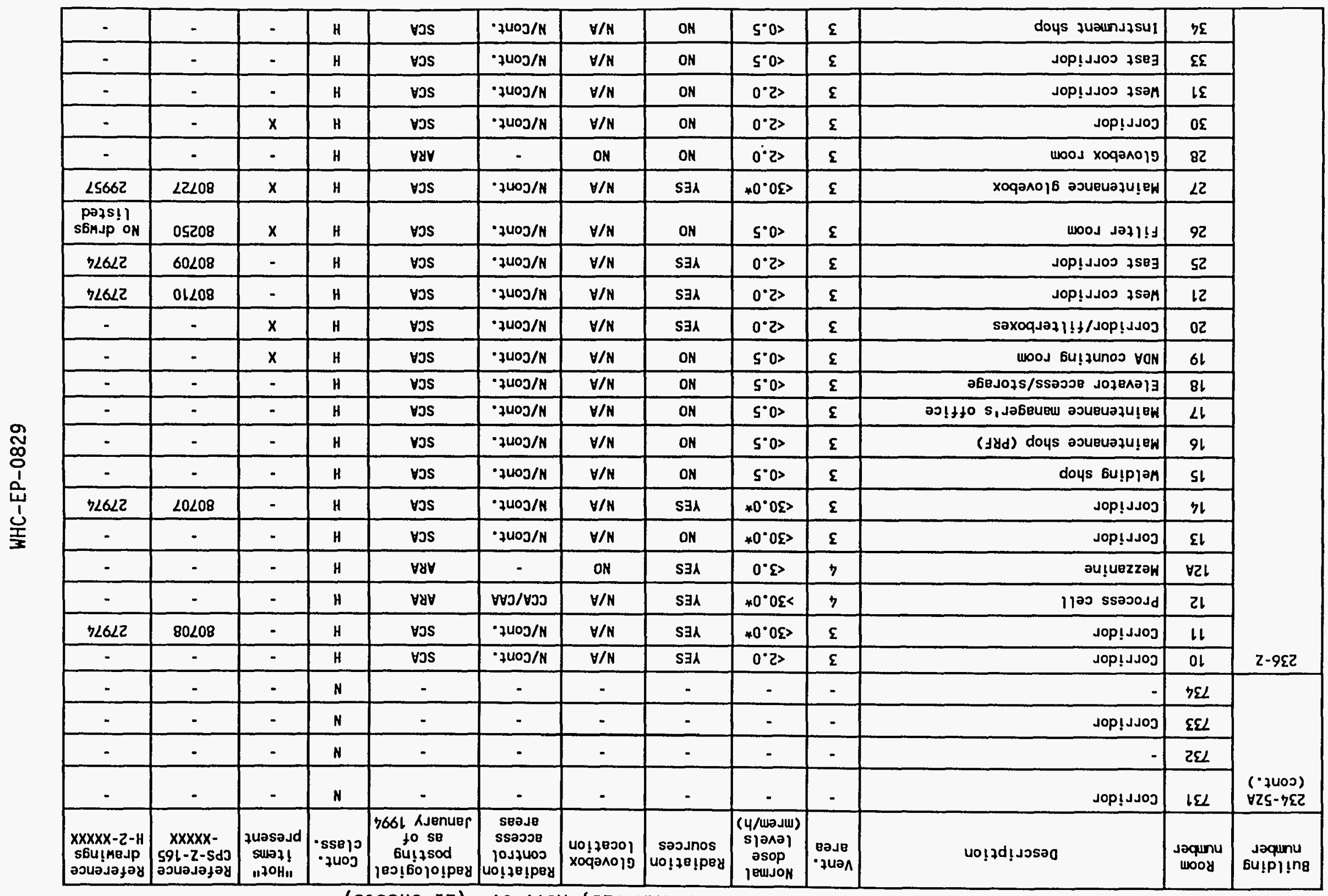

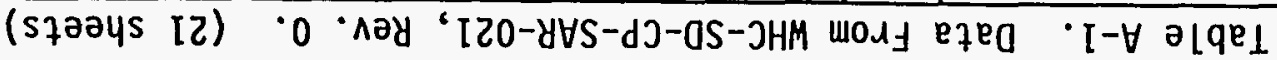


Table A-1. Data From HHC-SD-CP-SAR-021, Rev. 0. (21 sheets)

\begin{tabular}{|c|c|c|c|c|c|c|c|c|c|c|c|c|}
\hline $\begin{array}{l}\text { Building } \\
\text { number }\end{array}$ & $\begin{array}{c}\text { Room } \\
\text { number }\end{array}$ & Description & $\begin{array}{l}\text { Vent. } \\
\text { area }\end{array}$ & $\begin{array}{c}\text { Normal } \\
\text { dose } \\
\text { levels } \\
\text { (mrem/h) }\end{array}$ & $\begin{array}{l}\text { Radiation } \\
\text { sources }\end{array}$ & $\begin{array}{l}\text { Glovebox } \\
\text { location }\end{array}$ & $\begin{array}{c}\text { Radiation } \\
\text { control } \\
\text { access } \\
\text { areas }\end{array}$ & $\begin{array}{l}\text { Radiological } \\
\text { posting } \\
\text { as of } \\
\text { January } 1994\end{array}$ & $\begin{array}{l}\text { Cont. } \\
\text { class. }\end{array}$ & $\begin{array}{c}\text { "Hot" } \\
\text { items } \\
\text { present }\end{array}$ & $\begin{array}{c}\text { Reference } \\
\text { CPS-Z-165 } \\
-X X X X X \\
\end{array}$ & $\begin{array}{c}\text { Reference } \\
\text { draHings } \\
H-2-X X X X X\end{array}$ \\
\hline \multirow{16}{*}{$\begin{array}{l}236-2 \\
(\text { cont. })\end{array}$} & 35 & Electrical room & 3 & $<0.5$ & NO & N/A & N/Cont. & SCA & H & $x$ & - & - \\
\hline & 36 & Compressor room & 3 & $<0.5$ & No & H/A & H/Cont. & SCA & H & $x$ & $\cdot$ & - \\
\hline & 37 & Storage room & 3 & $<0.5$ & No & N/A & N/Cont. & SCA & H & - & - & - \\
\hline & 38 & Rest room & 3 & $<0.5$ & NO & N/A & N/Cont. & RCA & C & - & - & - \\
\hline & 40 & Chemical preparation & - & $<0.5$ & No & N/A & N/Cont. & SCA & H & $x$ & - & - \\
\hline & 41 & Miscellaneous treatment & - & $<30.0^{*}$ & YES & N/A & N/Cont. & SCA & H & $x$ & 80711 & 29872 \\
\hline & 42 & Column room & - & $<30.0 *$ & YES & N/A & N/Cont. & SCA & H & - & 80704 & 29885 \\
\hline & 43 & slag and crucible gloveboxes & - & $<2.0$ & YES & N/A & N/Cont. & RCA & $H$ & $x$ & 80731 & 29605 \\
\hline & $43 A$ & - & 3 & $<0.5$ & No & NO & - & SCA & c & - & $\cdot$ & $\cdot$ \\
\hline & 44 & Colum room & - & $<0.5$ & No & N/A & N/Cont. & RCA & C & $\cdot$ & - & - \\
\hline & 45 & Shift manager's office & - & $<0.5$ & NO & N/A & N/Cont. & RCA & C & - & - & $\cdot$ \\
\hline & 46 & Shift engineer's office & - & $<0.5$ & NO & N/A & N/Cont. & RCA & $\mathbf{c}$ & - & - & - \\
\hline & 47 & Corridor & - & $<0.5$ & No & N/A & N/Cont. & RCA & C & - & - & - \\
\hline & 50 & Colum glovebox & - & $<2.0$ & YES & N/A & N/Cont. & SCA & H & $x$ & 80705 & 29885 \\
\hline & 60 & Column glovebox & - & $<30.0$ & YES & N/A & N/Cont. & SCA & H & $x$ & 80705 & 29885 \\
\hline & 73 & Mechanical room & - & - & - & - & - & RCA & C & - & - & - \\
\hline $234-2 B$ & Bldg. & $\begin{array}{l}\text { Miscellaneous storage for } \\
\text { construction forces }\end{array}$ & 1 & N/A & NO & N/A & N/Cont. & CLEAR & N & $\cdot$ & $\cdot$ & - \\
\hline $234-2 C$ & Bldg. & $\begin{array}{l}\text { Loading dock for waste drums and } \\
\text { SNM containers }\end{array}$ & - & H/A & NO & N/A & N/Cont. & SCA/RCA & C & - & - & - \\
\hline $241-2$ & Bldg. & $\begin{array}{l}\text { Liquid waste collection tanks in an } \\
\text { underground concrete vault }\end{array}$ & 4 & $<0.5$ & YES & N/A & CCA/CAA & RCA & H & $x$ & 80741 & $\begin{array}{l}\text { No drwgs } \\
\text { listed }\end{array}$ \\
\hline $241-2-R B$ & Bldg. & $\begin{array}{l}\text { Haste water retention bas in (no } \\
\text { longer used) }\end{array}$ & - & H/A & - & N/A & - & $\cdot$ & H & - & - & - \\
\hline $241-2 A$ & Bldg. & 241-2 tank sampling & 4 & $<2.0$ & YES & H/A & - & RCA & H & $x$ & - & - \\
\hline $241-2 B$ & Bldg. & $\begin{array}{l}\text { Curbed concrete pad housing the D-9 } \\
\text { caustic tank }\end{array}$ & - & $N / A$ & - & N/A & - & RCA & N & $x$ & - & $\cdot$ \\
\hline $241-26$ & - & Change room & - & - & - & - & - & RCA & C & - & - & - \\
\hline
\end{tabular}


Table A-1. Data From WHC-SD-CP-SAR-021, Rev. 0. (21 sheets)

\begin{tabular}{|c|c|c|c|c|c|c|c|c|c|c|c|c|}
\hline $\begin{array}{l}\text { Building } \\
\text { number }\end{array}$ & $\begin{array}{c}\text { Room } \\
\text { number }\end{array}$ & Description & $\begin{array}{l}\text { Vent. } \\
\text { erea }\end{array}$ & $\begin{array}{c}\text { Hormal } \\
\text { dose } \\
\text { levels } \\
\text { (mrem/h) }\end{array}$ & $\begin{array}{l}\text { Radiation } \\
\text { sources }\end{array}$ & $\begin{array}{l}\text { Glovebox } \\
\text { location }\end{array}$ & \begin{tabular}{|c|} 
Radiation \\
control \\
access \\
areas
\end{tabular} & $\begin{array}{c}\text { Radiological } \\
\text { post ing } \\
\text { as of } \\
\text { January } 1994\end{array}$ & $\begin{array}{l}\text { Cont. } \\
\text { class. }\end{array}$ & $\begin{array}{c}\text { "Hot" } \\
\text { items } \\
\text { present }\end{array}$ & \begin{tabular}{c|} 
Reference \\
CPS $-z-165$ \\
$-X X X X X$
\end{tabular} & $\begin{array}{c}\text { Reference } \\
\text { drawings } \\
H-2-X X X X X\end{array}$ \\
\hline $241-2-361$ & Bldg. & $\begin{array}{l}\text { Underground liquid waste settling } \\
\text { tank (no longer used) }\end{array}$ & - & $\mathrm{N} / \mathrm{A}$ & - & $N / A$ & - & - & $\mathbf{N}$ & 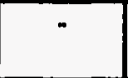 & - & - \\
\hline \multirow[t]{2}{*}{$242-2$} & Bldg. & $\begin{array}{l}\text { Waste Treatment facility (layaway } \\
\text { status) }\end{array}$ & 4 & N/A & YES & $N / A$ & CCA/CAA & SCA & H & $x$ & corr 242 & 43521 \\
\hline & 108 & Air lock & 1 & $<0.5$ & HO & - & N/Cont. & CLEAR & N & - & - & - \\
\hline $243-2 A$ & - & Tank and sump pit & - & - & - & - & - & - & c & - & - & - \\
\hline \multirow[t]{5}{*}{$243-z$} & 401 & - & - & - & - & - & - & - & C & - & - & - \\
\hline & 402 & - & - & - & - & 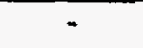 & - & 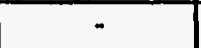 & $c$ & - & - & - \\
\hline & 403 & - & - & - & - & - & - & - & C & - & - & - \\
\hline & 404 & Drun washer & $\cdot$ & $\cdot$ & - & - & - & $\cdot$ & C & - & - & - \\
\hline & 405 & Drum storage & $\cdot$ & $\cdot$ & 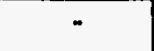 & - & - & - & $\mathbf{C}$ & - & - & - \\
\hline $243-28$ & $=$ & Cooling equipment pad & - & - & - & - & - & - & C & - & - & - \\
\hline $252 \cdot 2-1$ & Bldg. & Electrical transformers & - & H/A & - & H/A & - & $\cdot$ & H & - & - & - \\
\hline $252-z-2$ & Bldg. & Electrical transformers & 3 & H/A & - & H/A & - & CLEAR & C & - & - & - \\
\hline $267-2$ & Bldg. & $\begin{array}{l}\text { Valve house for water supply to a } \\
234-52 \text { sprinkler system }\end{array}$ & - & N/A & HO & N/A & - & CLEAR & H & $\cdot$ & - & $\overline{-}$ \\
\hline $270-2$ & Bldg. & $\begin{array}{l}\text { PRF support facility (office } \\
\text { building) }\end{array}$ & - & N/A & H/A & $N / A$ & $\cdot$ & $\cdot$ & H & $\cdot$ & - & - \\
\hline \multirow[t]{11}{*}{$291-2$} & Bldg. & Ventilation exhaust fan house & 3 & N/A & No & N/A & N/Cont. & RCA & C & $=$ & - & - \\
\hline & - & Cable vault & 3 & $<0.5$ & NO & N/A & N/Cont. & CLEAR & c & $\cdot$ & - & $\cdot$ \\
\hline & 500 & Electrical room & 3 & $<0.5$ & NO & N/A & N/Cont. & CLEAR & C & - & - & - \\
\hline & 501 & Compressor room & 3 & $<0.5$ & NO & N/A & N/Cont. & RCA \& SCA & c & $x$ & - & $\cdot$ \\
\hline & 502 & Exhaust plenum & 3 & $<0.5$ & No & $N / A$ & N/Cont. & RCA & C & $x$ & - & - \\
\hline & 503 & - & - & $\cdot$ & - & - & - & - & N & $\cdot$ & - & $\cdot$ \\
\hline & 504 & - & 3 & $<0.5$ & NO & N/A & N/Cont. & $\cdot$ & C & - & - & - \\
\hline & 505 & - & 3 & $<0.5$ & NO & N/A & N/Cont. & CLEAR & c & - & $\cdot$ & - \\
\hline & 506 & - & 3 & $<0.5$ & NO & $N / A$ & N/Cont. & CLEAR & C & - & $\cdot$ & - \\
\hline & 507 & - & 3 & $<0.5$ & NO & N/A & N/Cont. & CLEAR & C & - & - & - \\
\hline & 508 & - & 3 & $<0.5$ & NO & N/A & N/Cont. & CLEAR & C & - & - & - \\
\hline
\end{tabular}


Table A-1. Data From WHC-SD-CP-SAR-021, Rev. 0. (21 sheets)

\begin{tabular}{|c|c|c|c|c|c|c|c|c|c|c|c|c|}
\hline $\begin{array}{l}\text { Building } \\
\text { number }\end{array}$ & $\begin{array}{c}\text { Room } \\
\text { number }\end{array}$ & Description & $\begin{array}{c}\text { Vent. } \\
\text { area }\end{array}$ & $\begin{array}{c}\text { Normal } \\
\text { dose } \\
\text { levels } \\
\text { (mrem/h) }\end{array}$ & $\begin{array}{c}\text { Radiation } \\
\text { sources }\end{array}$ & $\begin{array}{l}\text { Glovebox } \\
\text { location }\end{array}$ & \begin{tabular}{|c|} 
Radiation \\
control \\
access \\
areas
\end{tabular} & \begin{tabular}{|c|} 
Rediological \\
posting \\
as of \\
January 1994
\end{tabular} & $\begin{array}{c}\text { Cont. } \\
\text { class. }\end{array}$ & \begin{tabular}{|c|} 
"Hot" \\
items \\
present
\end{tabular} & \begin{tabular}{|c|} 
Reference \\
CPS $-z-165$ \\
$-X X X X X$
\end{tabular} & $\begin{array}{l}\text { Reference } \\
\text { drawings } \\
H-2=x X X X X\end{array}$ \\
\hline \multirow{2}{*}{$\begin{array}{l}291-2 \\
\text { (cont.) }\end{array}$} & 509 & Fan room & 3 & $<0.5$ & NO & N/A & H/Cont. & RCA & c & $\overline{-}$ & - & $\cdot$ \\
\hline & 510 & - & - & - & - & - & - & - & N & - & - & - \\
\hline $291-2-1$ & Bldg. & $\begin{array}{l}\text { Ventilation exhaust stack for } \\
232-2,234-52 \text {, and } 242-2 \text { Buildings }\end{array}$ & 3 & N/A & No & H/A & - & CLEAR & C & - & - & - \\
\hline $296-2-3$ & Bldg. & $\begin{array}{l}\text { Ventilation exhaust stack for } 241-2 \\
\text { vaul } t \text { and tanks }\end{array}$ & - & $N / A$ & - & N/A & - & - & N & - & - & - \\
\hline $296-z-5$ & Bldg. & $\begin{array}{l}\text { Ventilation exhaust stack for } \\
2736-2 \text { Building }\end{array}$ & - & N/A & - & N/A & - & - & N & - & - & - \\
\hline $296-2-6$ & Bldg. & $\begin{array}{l}\text { Ventilation exhaust stack for } \\
2736-2 \text { Building }\end{array}$ & - & N/A & - & N/A & - & - & $\mathbf{N}$ & - & - & - \\
\hline $2503-2$ & Bldg. & $\begin{array}{l}\text { Electrical distribution system for } \\
\text { PFP (switch yard) }\end{array}$ & - & N/A & N/A & N/A & - & - & N & - & - & - \\
\hline $2709-2$ & Bldg. & Mooden building (no longer used) & - & N/A & N/A & N/A & - & - & $\mathbf{N}$ & - & - & - \\
\hline $2701-2 A$ & Bldg. & Central station alarm facility & - & N/A & N/A & $H / A$ & - & CLEAR & H & - & - & - \\
\hline $2701-2 B$ & Bldg. & Security badge house & - & N/A & $\mathrm{N} / \mathrm{A}$ & $\mathrm{N} / \mathrm{A}$ & - & - & N & - & - & - \\
\hline $2701-2 D$ & Bldg. & Security checkpoint station & - & - & - & - & - & - & - & - & - & - \\
\hline $2702-2$ & Bldg. & Microwave tower & - & N/A & $\mathrm{H} / \mathrm{A}$ & $N / A$ & - & - & H & - & - & - \\
\hline $2704-2$ & Bldg. & $\begin{array}{l}\text { PFP support facility (office } \\
\text { building) }\end{array}$ & - & N/A & - & N/A & - & CLEAR & N & - & - & - \\
\hline $2705-2$ & Bldg. & Badge house & - & - & - & - & - & - & - & - & - & - \\
\hline $2712-2$ & Bldg. & $\begin{array}{l}\text { 291-2-1 stack sampler power } \\
\text { generators (diesel driven) }\end{array}$ & 3 & N/A & No & $N / A$ & - & - & c & - & - & - \\
\hline $2715-2$ & Bldg. & Paint and solvent storage & - & N/A & N/A & N/A & - & CLEAR & N & - & - & - \\
\hline $2715-\mathrm{ZL}$ & Bldg. & Drum storage & - & N/A & H/A & H/A & - & - & H & - & - & - \\
\hline $2721-2$ & Bldg. & $\begin{array}{l}\text { Emergency electric power generators } \\
\text { (diesel driven) }\end{array}$ & 1 & N/A & Ho & $H / A$ & $\mathrm{~N} /$ Cont. & CLEAR & H & $\cdot$ & - & - \\
\hline $2722-2$ & Bldg. & $\begin{array}{l}\text { Concrete pad for truck load-out } \\
\text { station }\end{array}$ & 1 & $N / A$ & NO & $N / A$ & - & - & N & - & - & - \\
\hline $2725-2$ & Bldg. & Laundry storage & 1 & N/A & NO & N/A & $H /$ Cont. & CLEAR & $H$ & - & - & - \\
\hline $2727-2$ & Bldg. & $\begin{array}{l}\text { Storage of miscellaneous operations } \\
\text { and laboratory equipment }\end{array}$ & 1 & N/A & - & H/A & - & CLEAR & H & - & - & - \\
\hline $2729-2$ & Bldg. & $\begin{array}{l}\text { Storage of miscellaneous } \\
\text { maintenance materials }\end{array}$ & $\cdot$ & N/A & $H / A$ & $\mathrm{H} / \mathrm{A}$ & - & CLEAR & H & - & - & - \\
\hline
\end{tabular}


Table A-1. Data From WHC-SD-CP-SAR-021, Rev. 0. (21 sheets)

\begin{tabular}{|c|c|c|c|c|c|c|c|c|c|c|c|c|}
\hline $\begin{array}{l}\text { Building } \\
\text { number }\end{array}$ & $\begin{array}{c}\text { Room } \\
\text { number }\end{array}$ & Description & $\begin{array}{l}\text { Vent. } \\
\text { area }\end{array}$ & $\begin{array}{c}\begin{array}{c}\text { Normal } \\
\text { dose } \\
\text { levels } \\
\text { (mrem/h) }\end{array} \\
\end{array}$ & $\begin{array}{l}\text { Radiation } \\
\text { sources }\end{array}$ & $\begin{array}{l}\text { Glovebox } \\
\text { location }\end{array}$ & $\begin{array}{c}\text { Radiation } \\
\text { control } \\
\text { access } \\
\text { areas }\end{array}$ & \begin{tabular}{|c|} 
Radiological \\
post ting \\
os of \\
January 9994
\end{tabular} & $\begin{array}{l}\text { Cont. } \\
\text { class. }\end{array}$ & \begin{tabular}{|c|} 
"Hot" \\
items \\
present
\end{tabular} & \begin{tabular}{|c|} 
Reference \\
CPS- -165 \\
$-X X X X X$
\end{tabular} & $\begin{array}{c}\text { Reference } \\
\text { drawings } \\
H-2-X X X X X\end{array}$ \\
\hline $2731-2$ & Bldg. & $\begin{array}{l}\text { Storage of empty flushed plutonium } \\
\text { drums }\end{array}$ & 1 & N/A & No & N/A & N/Cont. & CLEAR & $N$ & - & 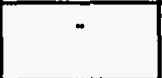 & $\overline{-}$ \\
\hline $2731-2 A$ & Bldg. & Laundry storage & 1 & $\mathrm{H} / \mathrm{A}$ & No & N/A & N/Cont. & RCA & C & - & - & - \\
\hline $2734-2$ & Bldg. & Gas bottle storage & - & N/A & $\overline{-}$ & $\overline{N / A}$ & - & - & N & - & - & - \\
\hline $2734-2 A$ & Bldg. & Liquid argon storage & 1 & N/A & No & N/A & - & CLEAR & $N$ & - & - & - \\
\hline $2734-2 B$ & Bldg. & $\begin{array}{l}\begin{array}{l}\text { Flammable gas storage (out of } \\
\text { service) }\end{array} \\
\end{array}$ & 1 & N/A & NO & N/A & - & CLEAR & N & - & - & - \\
\hline $2734-2 C$ & Bldg. & Gas bottle storage (out of service) & 1 & N/A & No & N/A & - & RCA & H & - & $\overline{-}$ & - \\
\hline $2734-20$ & Bldg. & $\begin{array}{l}\text { Argon and oxygen bottle supply (out } \\
\text { of service) }\end{array}$ & 1 & $N / A$ & NO & $N / A$ & - & CLEAR & N & $\overline{-}$ & - & - \\
\hline $2734-2 F$ & Bldg. & $\begin{array}{l}\text { Standby gas bottle supply (out of } \\
\text { service) }\end{array}$ & 1 & H/A & NO & $N / A$ & $\overline{-}$ & CLEAR & $N$ & - & - & - \\
\hline $2734-26$ & Bldg. & \begin{tabular}{|l}
$\begin{array}{l}\text { Standby gas bottle supply (out of } \\
\text { service) }\end{array}$ \\
\end{tabular} & 1 & $N / A$ & NO & $N / A$ & - & CLEAR & H & - & - & - \\
\hline $2734-2 H$ & Bldg. & $\begin{array}{l}\text { Argon bottle supply (out of } \\
\text { service) }\end{array}$ & 1 & $H / A$ & NO & N/A & - & CLEAR & N & - & - & - \\
\hline $2734-2 \mathrm{~J}$ & Bldg. & Liquid nitrogen storage tank & 1 & $\mathrm{H} / \mathrm{A}$ & NO & N/A & - & CLEAR & M & - & - & - \\
\hline $2734-2 K$ & Bldg. & $\begin{array}{l}\text { Gas bottle storage, acetylene, } \\
\text { argon, oxygen }\end{array}$ & 1 & N/A & No & N/A & - & CLEAR & N & - & 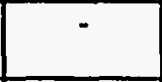 & - \\
\hline $2734-2 L$ & Bldg. & HF gas bottles and supply piping & 1 & $N / A$ & No & N/A & CAA & CLEAR & C & - & - & - \\
\hline $2735-2$ & Bldg. & Chemical storage of $\mathrm{HNO}_{3}$, and ANN & 1 & N/A & No & $\mathrm{H} / \mathrm{A}$ & N/Cont. & CLEAR & $N$ & - & - & - \\
\hline \multirow[t]{5}{*}{$2736-2$} & Bldg. & SNM storage & 3 & N/A & YES & N/A & YES & RCA & $H$ & - & - & - \\
\hline & 1 & Storage of product/recoverable SNM & 3 & $<30.0$ & YES & H/A & CAA & RCA & H & $\cdot$ & 80102 & $\cdot$ \\
\hline & 2 & Lard can/cubicle SNM storage & 3 & $>30.0$ & YES & H/A & CAA & RCA & $H$ & $\cdot$ & $\cdot$ & - \\
\hline & 3 & Storage of product/recoverable SNM & 3 & $>30.0$ & YES & N/A & CMA & RCA & $H$ & - & - & - \\
\hline & 4 & Storage of product/recoverable SNM & 3 & $>30.0$ & YES & $\mathrm{H} / \mathrm{A}$ & CAn & RCA & H & $\dot{-}$ & - & - \\
\hline $2736-2 A$ & Bldg. & $\begin{array}{l}2736-2 \text { exhaust fans and } 65-\mathrm{kW} \\
\text { diesel generator }\end{array}$ & 1 & N/A & No & H/A & M/Cont. & CLEAR & H & $x$ & - & - \\
\hline \multirow[t]{4}{*}{$2736-2 B$} & Bldg. & Shipping and receiving SNM & 3 & N/A & YES & N/A & YES & N/A & H & - & - & - \\
\hline & 600 & Mechanical room & 1 & $<0.5$ & NO & N/A & N/Cont. & CLEAR & H & $x$ & - & - \\
\hline & 602 & Mechanical room & 1 & $<0.5$ & No & N/A & N/Cont. & CLEAR & N & - & - & - \\
\hline & 603 & - & 1 & $<0.5$ & HO & $H / A$ & $\mathrm{H} /$ Cont. & CLEAR & H & - & - & - \\
\hline
\end{tabular}


Table A-1. Data From WHC-SD-CP-SAR-021, Rev. 0. (21 sheets)

\begin{tabular}{|c|c|c|c|c|c|c|c|c|c|c|c|c|}
\hline $\begin{array}{l}\text { Building } \\
\text { number }\end{array}$ & $\begin{array}{c}\text { Room } \\
\text { number }\end{array}$ & Description & $\begin{array}{l}\text { Vent. } \\
\text { area }\end{array}$ & 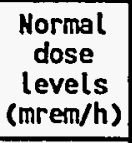 & $\begin{array}{c}\text { Radiation } \\
\text { sources }\end{array}$ & $\begin{array}{l}\text { Glovebox } \\
\text { location }\end{array}$ & \begin{tabular}{|c|}
$\begin{array}{c}\text { Radiation } \\
\text { control } \\
\text { access } \\
\text { areas }\end{array}$ \\
\end{tabular} & \begin{tabular}{|c|} 
Radiological \\
posting \\
as of \\
January 1994 \\
\end{tabular} & $\begin{array}{l}\text { Cont. } \\
\text { class. }\end{array}$ & \begin{tabular}{|c|} 
"Hot" \\
items \\
present
\end{tabular} & \begin{tabular}{|c|} 
Reference \\
CPS-z-165 \\
$-X X X X X X$ \\
\end{tabular} & \begin{tabular}{|c|} 
Reference \\
drawings \\
$H-2-x x x x x X$
\end{tabular} \\
\hline \multirow[t]{25}{*}{$\begin{array}{l}2736-2 B \\
\text { (cont.) }\end{array}$} & 604 & Security computer room & 1 & $<0.5$ & NO & N/A & N/Cont. & CLEAR & N & - & - & - \\
\hline & 605 & Office & 1 & $<0.5$ & NO & $N / A$ & N/Cont. & CLEAR & $N$ & - & - & - \\
\hline & 606 & Office & 1 & $<0.5$ & No & N/A & H/Cont. & CLEAR & N & - & - & - \\
\hline & 607 & Office & 1 & $<0.5$ & NO & N/A & N/Cont. & CLEAR & N & - & - & - \\
\hline & 608 & Vestibule & 1 & $<0.5$ & NO & N/A & N/Cont. & CLEAR & $\mathrm{N}$ & - & - & - \\
\hline & 610 & Receptionist's office & 1 & $<0.5$ & NO & N/A & N/Cont. & CLEAR & N & - & - & - \\
\hline & 611 & Office & 1 & $<0.5$ & NO & N/A & H/Cont. & $\overline{\text { CLEAR }}$ & $\bar{N}$ & - & - & - \\
\hline & 612 & Office & 1 & $<0.5$ & NO & N/A & N/Cont. & CLEAR & $\mathrm{N}$ & - & - & - \\
\hline & 613 & - & 1 & $<0.5$ & No & N/A & H/Cont. & CLEAR & $\mathrm{N}$ & - & - & - \\
\hline & 614 & Homen's change room & 1 & $<0.5$ & NO & N/A & N/Cont. & CLEAR & $\mathbf{N}$ & - & - & - \\
\hline & 615 & Homen's change room & 1 & $<0.5$ & NO & N/A & N/Cont. & CLEAR & $N$ & - & $\overline{-}$ & - \\
\hline & 616 & Homen's change room & 1 & $<0.5$ & NO & N/A & H/Cont. & CLEAR & N & - & - & - \\
\hline & 618 & Men's change room & 1 & $<0.5$ & NO & N/A & H/Cont. & CLEAR & N & $\overline{-}$ & - & - \\
\hline & 619 & Men's change room & 1 & $<0.5$ & NO & N/A & N/Cont. & CLEAR & H & - & - & - \\
\hline & 620 & Lunchroom & 1 & $<0.5$ & No & N/A & N/Cont. & CLEAR & H & $\overline{-}$ & - & - \\
\hline & 621 & Men's change room & 1 & $<0.5$ & No & $\mathrm{N} / \mathrm{A}$ & N/Cont. & CLEAR & $N$ & - & - & $\cdot$ \\
\hline & 622 & Men's change room & 1 & $<0.5$ & No & N/A & N/Cont. & CLEAR & N & $\overline{-}$ & - & - \\
\hline & 623 & \begin{tabular}{|l} 
UPS room \\
\end{tabular} & 1 & $<0.5$ & No & N/A & H/Cont. & CLEAR & N & - & - & - \\
\hline & 624 & \begin{tabular}{|l|} 
Corridor \\
\end{tabular} & 1 & $<0.5$ & No & N/A & N/Cont. & CLEAR & H & - & - & - \\
\hline & 625 & Corridor & 3 & $<0.5$ & No & $N / A$ & N/Cont. & RCA & $\bar{c}$ & - & - & - \\
\hline & 626 & Janitors' closet & 3 & $<0.5$ & NO & N/A & H/Cont. & RCA & $\bar{c}$ & - & - & $\cdot$ \\
\hline & 627 & Homen's rest room & 3 & $<0.5$ & HO & $H / A$ & s/Cont. & RCA & $\bar{c}$ & - & - & - \\
\hline & 628 & Men's rest room & 3 & $<0.5$ & HO & $N / A$ & H/Cont. & $\mathrm{RCA}$ & c & $\overline{-}$ & - & - \\
\hline & 629 & Mardix & 3 & $<0.5$ & HO & N/A & H/Cont. & CLEAR & C & - & - & - \\
\hline & 630 & Decontamination room & 3 & $<0.5$ & HO & K/A & H/Cont. & RCA & $\mathbf{c}$ & $x$ & - & - \\
\hline
\end{tabular}


Table A-1. Data From WHC-SD-CP-SAR-021, Rev. 0. (21 sheets)

\begin{tabular}{|c|c|c|c|c|c|c|c|c|c|c|c|c|}
\hline $\begin{array}{l}\text { Building } \\
\text { number }\end{array}$ & $\begin{array}{l}\text { Room } \\
\text { number }\end{array}$ & Description & $\begin{array}{l}\text { Vent. } \\
\text { area }\end{array}$ & $\begin{array}{c}\text { Normal } \\
\text { dose } \\
\text { levels } \\
(\mathrm{mrem} / \mathrm{h})\end{array}$ & $\begin{array}{l}\text { Radiation } \\
\text { sources }\end{array}$ & $\begin{array}{l}\text { Glovebox } \\
\text { location }\end{array}$ & $\begin{array}{c}\text { Radiation } \\
\text { control } \\
\text { access } \\
\text { areas }\end{array}$ & $\begin{array}{l}\text { Radiological } \\
\text { posting } \\
\text { as of } \\
\text { January } 1994 \\
\end{array}$ & $\begin{array}{l}\text { Cont. } \\
\text { class. }\end{array}$ & $\begin{array}{c}\text { "Hot" } \\
\text { items } \\
\text { present }\end{array}$ & $\begin{array}{c}\text { Reference } \\
\text { CPS-Z-165 } \\
-X X X X X\end{array}$ & $\begin{array}{c}\text { Reference } \\
\text { drawings } \\
H-2-x x x X X X\end{array}$ \\
\hline \multirow{18}{*}{$\begin{array}{l}2736-28 \\
\text { (cont.) }\end{array}$} & 631 & Heal th Physics office & 3 & $<0.5$ & NO & N/A & H/Cont. & RCA & C & - & - & - \\
\hline & 632 & Applied technology chemist's office & 3 & $<0.5$ & NO & N/A & N/Cont. & RCA & C & - & - & - \\
\hline & 633 & Corridor & 3 & $<0.5$ & NO & N/A & W/Cont. & RCA & C & - & $\overline{-}$ & - \\
\hline & 634 & Shift manager & 3 & $<0.5$ & NO & $N / A$ & H/Cont. & RCA & C & - & - & - \\
\hline & 635 & Operator ready room & 3 & $<0.5$ & NO & N/A & N/Cont. & RCA & C & - & - & - \\
\hline & 636 & Repackaging room & 4 & $<30.0 *$ & YES & H/A & H/Cont. & RCA & H & $x$ & 80171 & 80174 \\
\hline & 637 & NDA laboratory & 3 & $<30.0^{\star}$ & YES & N/A & N/Cont. & RCA & H & $x$ & 80121 & $\begin{array}{l}\text { No drwgs } \\
\text { listed }\end{array}$ \\
\hline & 638 & Packaging room & 3 & $<30.0^{\star}$ & YES & N/A & CAA & RCA & $H$ & $x$ & 80360 & $\begin{array}{l}\text { No drwgs } \\
\text { listed }\end{array}$ \\
\hline & $638 \mathrm{~A}$ & - & 3 & $<30.0^{*}$ & YES & H/A & CAA & RCA & H & $\cdot$ & $\cdot$ & - \\
\hline & 639 & Safety shower room & 3 & $<30.0 \star$ & NO & $N / A$ & CAA & RCA & $H$ & $x$ & - & - \\
\hline & 640 & Supply storage & 3 & $<30.0^{\star}$ & NO & H/A & CAA & RCA & $H$ & - & - & - \\
\hline & 641 & Receiving room & 3 & $<30.0^{\star}$ & NO & N/A & CAA & RCA & H & $x$ & - & - \\
\hline & $641 \mathrm{~A}$ & - & 3 & $<30.0^{*}$ & YES & N/A & CAA & RCA & H & - & - & $=$ \\
\hline & 642 & Shipping room & 3 & $<30.0^{*}$ & YES & N/A & CAA & RCA & $H$ & $x$ & - & - \\
\hline & 643 & Personnel entry & 3 & $<0.5$ & NO & N/A & H/Cont. & RCA & c & - & - & - \\
\hline & 644 & Material passageway & 3 & $<0.5$ & NO & N/A & N/Cont. & RCA & C & $\cdot$ & $\cdot$ & - \\
\hline & 645 & - & 1 & $<0.5$ & No & N/A & N/Cont. & CLEAR & N & - & - & - \\
\hline & 646 & - & 1 & $<0.5$ & NO & H/A & N/Cont. & CLEAR & N & - & - & - \\
\hline $2736-2 c$ & Bldg. & Loading dock & 1 & $H / A$ & HO & H/A & H/Cont. & CLEAR & H & - & - & - \\
\hline $2902-2$ & Bldg. & $\begin{array}{l}\text { Elevated sanitary water tank and } \\
\text { valve pit }\end{array}$ & - & $N / A$ & N/A & N/A & - & $N / A$ & N & - & - & - \\
\hline $2904-2 A$ & Bldg. & $\begin{array}{l}\text { Sheet metal structure housing } \\
216-2-20 \text { crib stream effluent } \\
\text { sampl ing, flow measurement, pH, and } \\
\text { al pha monitoring equipment }\end{array}$ & - & N/A & N/A & N/A & - & $N / A$ & N & - & - & - \\
\hline
\end{tabular}


Table A-1. Data From WHC-SD-CP-SAR-021, Rev. 0. (21 sheets)

\begin{tabular}{|c|c|c|c|c|c|c|c|c|c|c|c|c|}
\hline $\begin{array}{l}\text { Building } \\
\text { number }\end{array}$ & $\begin{array}{c}\text { Room } \\
\text { number }\end{array}$ & Description & $\begin{array}{l}\text { Vent. } \\
\text { area }\end{array}$ & $\begin{array}{c}\text { Normal } \\
\text { dose } \\
\text { levels } \\
\text { (mrem/h) }\end{array}$ & $\begin{array}{l}\text { Radiation } \\
\text { sources }\end{array}$ & $\begin{array}{l}\text { Glovebox } \\
\text { location }\end{array}$ & $\begin{array}{c}\text { Radiation } \\
\text { control } \\
\text { access } \\
\text { areas }\end{array}$ & $\begin{array}{l}\text { Radiological } \\
\text { post ing } \\
\text { as of } \\
\text { January } 1994\end{array}$ & $\begin{array}{l}\text { Cont. } \\
\text { class. }\end{array}$ & $\begin{array}{c}\text { "Hot" } \\
\text { items } \\
\text { present }\end{array}$ & $\begin{array}{c}\text { Reference } \\
\text { CPS }-2-165 \\
-x x x x x\end{array}$ & $\begin{array}{l}\text { Reference } \\
\text { drawings } \\
H-2-X X X X X\end{array}$ \\
\hline $2904-2 B$ & Bldg. & $\begin{array}{l}\text { Sheet metal structure housing } \\
216-2-20 \mathrm{crib} \text { stream effluent } \\
\text { sampl ing, flow measurement, pH, and } \\
\text { alpha monitoring equipment }\end{array}$ & - & H/A & H/A & N/A & - & N/A & $\bar{N}$ & - & - & - \\
\hline
\end{tabular}

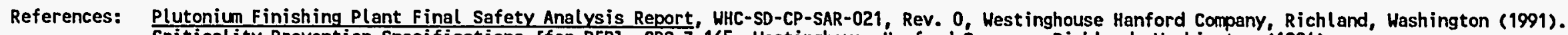
Criticality Prevention Specifications [for PFPI, CPS-2-165, Westinghouse Hanford Company, Richland, Washington (1994).

ANN = Aluminum ni trate nonhydrate.

$H F=$ Hydrofluoric acid.

IRM = Information Resource Management (Department).

NDA $a$ Hondestructive assay.

PR = Product removal.

PFP = Plutonium Finishing Plant.

PFP $=$ Plutonium Finishing Plant
PRF

RADTU = Radioactive Acid Digestion Test Unit.

RCA = Radiological controlled area.

RMA = Remote mechanical "A" line.

RMC = Remote mechanical "C" line.

RHP = Radiological Hork permit.

SNM = Special nuclear material.

SSDM = structured system development methodology (data management).

SWP = Special work permit (protective clothing).

UPS = Uninterruptible power supply.

HIPP $=$ Waste Isolation Pi lot Plant. 
WHC-EP-0829

This page intentionally left blank. 
WHC-EP-0829

APPENDIX B

ESTIMATION OF PIPING REMOVAL DURING DECONTANINATION AND DECOMMISSIONING AT THE PLUTONIUH FINISHING PLANT 
WHC-EP-0829

This page intentionally left blank. 
ESTIMATION OF PIPING REMOVAL DURING DECONTAMINATION AND DECOMMISSIONING

AT THE PLUTONIUM FINISHING PLANT

This appendix contains the spreadsheet (generated in Excel 5.0) that was used to estimate the volume of piping external to gloveboxes and hoods that is likely to be removed during the decontamination and decommissioning of the Plutonium Finishing Plant. 
Table B-1. Drain Pipe Dimensions for the 234-5Z Building. (2 sheets)

\begin{tabular}{|c|c|c|c|c|c|c|c|c|}
\hline \multirow{2}{*}{ Line } & \multicolumn{2}{|c|}{ Length } & \multicolumn{2}{|c|}{ Diameter } & \multicolumn{2}{|c|}{ Volume } & \multicolumn{2}{|c|}{ Total } \\
\hline & $f t$ & $\% m$ & in.. & $\mathrm{cm}$. & $\sin ^{3} \theta$ & $m^{3}:$ & $8\left(\operatorname{in}^{3}\right) \cdots$ & $\%\left(\mathrm{~m}^{3}\right) \mathrm{M}$ \\
\hline \multirow[t]{4}{*}{ D-1 acid drain in $234-52$} & 2 & 0.61 & 4 & 10.16 & 301.44 & 0.005 & \multirow[b]{4}{*}{$9,467.10$} & \multirow[b]{4}{*}{0.155} \\
\hline & 82 & 24.99 & 3 & 7.62 & $6,951.96$ & 0.114 & & \\
\hline & 52 & 15.85 & 2 & 5.08 & $1,959.36$ & 0.032 & & \\
\hline & 12 & 3.66 & 9.5 & 3.81 & 254.34 & 0.004 & & \\
\hline \multirow[t]{3}{*}{$0-1$ line in trench } & 30 & 9.14 & 3 & 7.62 & $2,543.40$ & 0.042 & \multirow[b]{3}{*}{$1,792,343.40$} & \multirow[b]{3}{*}{29.371} \\
\hline & 100 & 30.48 & 10 & 25.40 & $94,200.00$ & 1.544 & & \\
\hline & 800 & 243.84 & 15 & 38.10 & $1,695,600.00$ & 27.786 & & \\
\hline \multirow[t]{4}{*}{$0-2$ clear water drain in $234-52$} & 513 & 156.36 & 4 & 10.16 & $77,319.36$ & 1.267 & \multirow[b]{4}{*}{$124,560.66$} & \multirow[b]{4}{*}{2.041} \\
\hline & 449 & 136.86 & 3 & 7.62 & $38,066.22$ & 0.624 & & \\
\hline & 167 & 50.90 & 2 & 5.08 & $6,292.56$ & 0.103 & & \\
\hline & 136 & 41.45 & 1.5 & 3.81 & $2,882.52$ & 0.047 & & \\
\hline $0-2$ line in trench & 30 & 9.14 & 4 & 10.16 & $4,521.60$ & 0.074 & $4,521.60$ & 0.074 \\
\hline$D-1$ and $D-2$ combined line & 820 & 249.94 & 15 & 38.1 & $1,737,990.00$ & 28.480 & $1,737,990.00$ & 28.480 \\
\hline \multirow[t]{5}{*}{ D-3 process cooling drain in $234-52$} & 60 & 18.29 & 6 & 15.24 & $20,347.20$ & 0.333 & \multirow[b]{5}{*}{$86,802.95$} & \multirow[b]{5}{*}{1.422} \\
\hline & 163 & 49.68 & 4 & 10.16 & $24,567.36$ & 0.403 & & \\
\hline & 290 & 88.39 & 3 & 7.62 & $24,586.20$ & 0.403 & & \\
\hline & 237 & 72.24 & 2 & 5.08 & $8,930.16$ & 0.146 & & \\
\hline & 395 & 120.40 & 1.5 & 3.81 & $8,372.03$ & 0.137 & & \\
\hline D-3 line to retention pond & 400 & 121.92 & 8 & 20.32 & 241.152 .00 & 3.952 & $241,152.00$ & 3.952 \\
\hline \multirow{3}{*}{$\begin{array}{l}\text { D-4 Development Laboratory drain } \\
\text { in } 234-52\end{array}$} & 417 & 127.10 & 4 & 10.16 & $62,850.24$ & 1.030 & \multirow[b]{3}{*}{$102,291.78$} & \multirow[b]{3}{*}{1.676} \\
\hline & 463 & 141.12 & 3 & 7.62 & $39,253.14$ & 0.643 & & \\
\hline & 5 & 1.52 & 2 & 5.08 & 188.40 & 0.003 & & \\
\hline $0-4$ in trench to $241-2$ & 320 & 97.54 & 4 & 10.16 & $48,230.40$ & 0.790 & $48,230.40$ & 0.790 \\
\hline $0-4$ in $241-2$ & 10 & 3.05 & 4 & 10.16 & $1,507.20$ & 0.025 & $1,507.20$ & 0.025 \\
\hline
\end{tabular}


Table B-1. Drain Pipe Dimensions for the 234-5Z Building. (2 sheets)

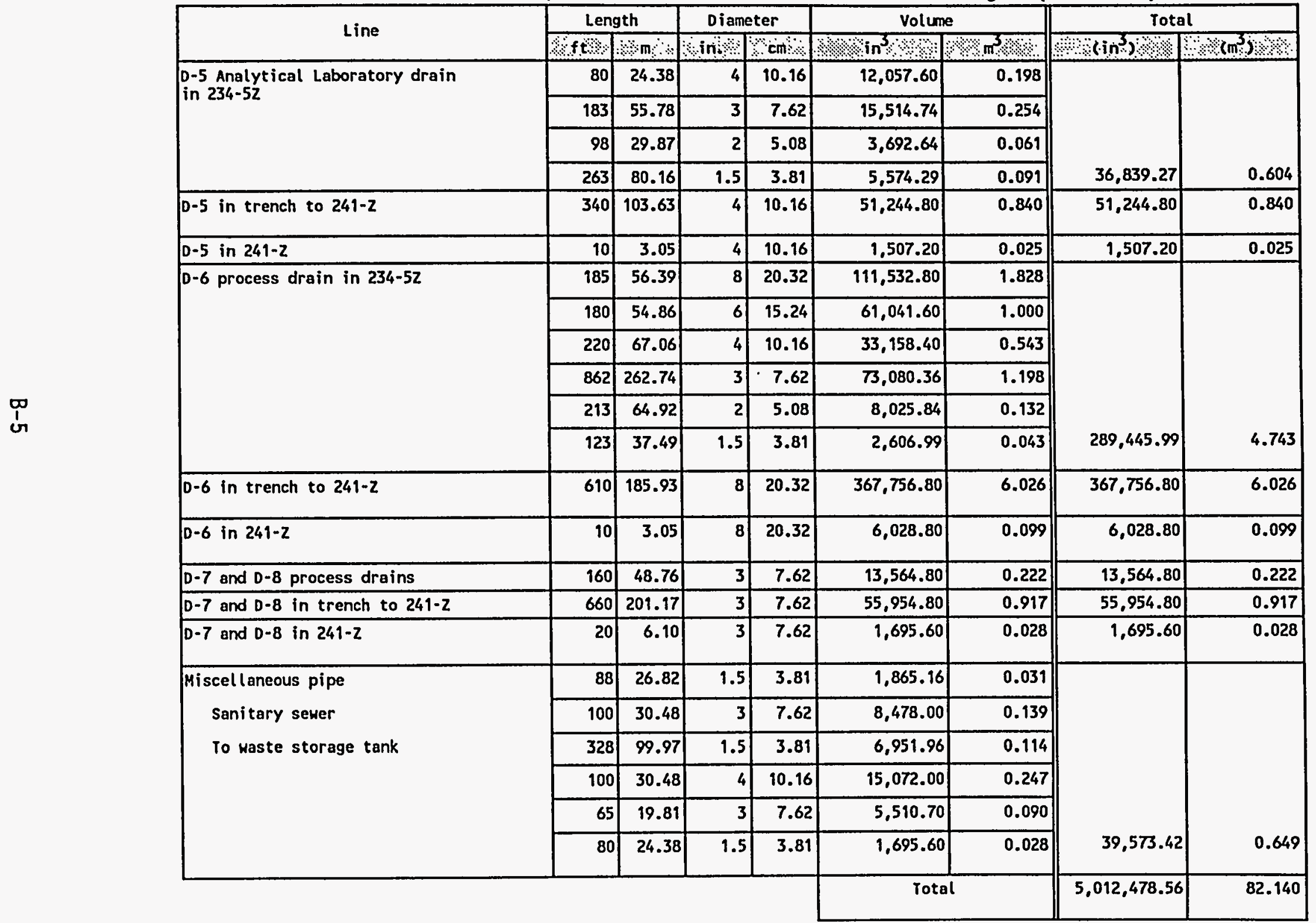




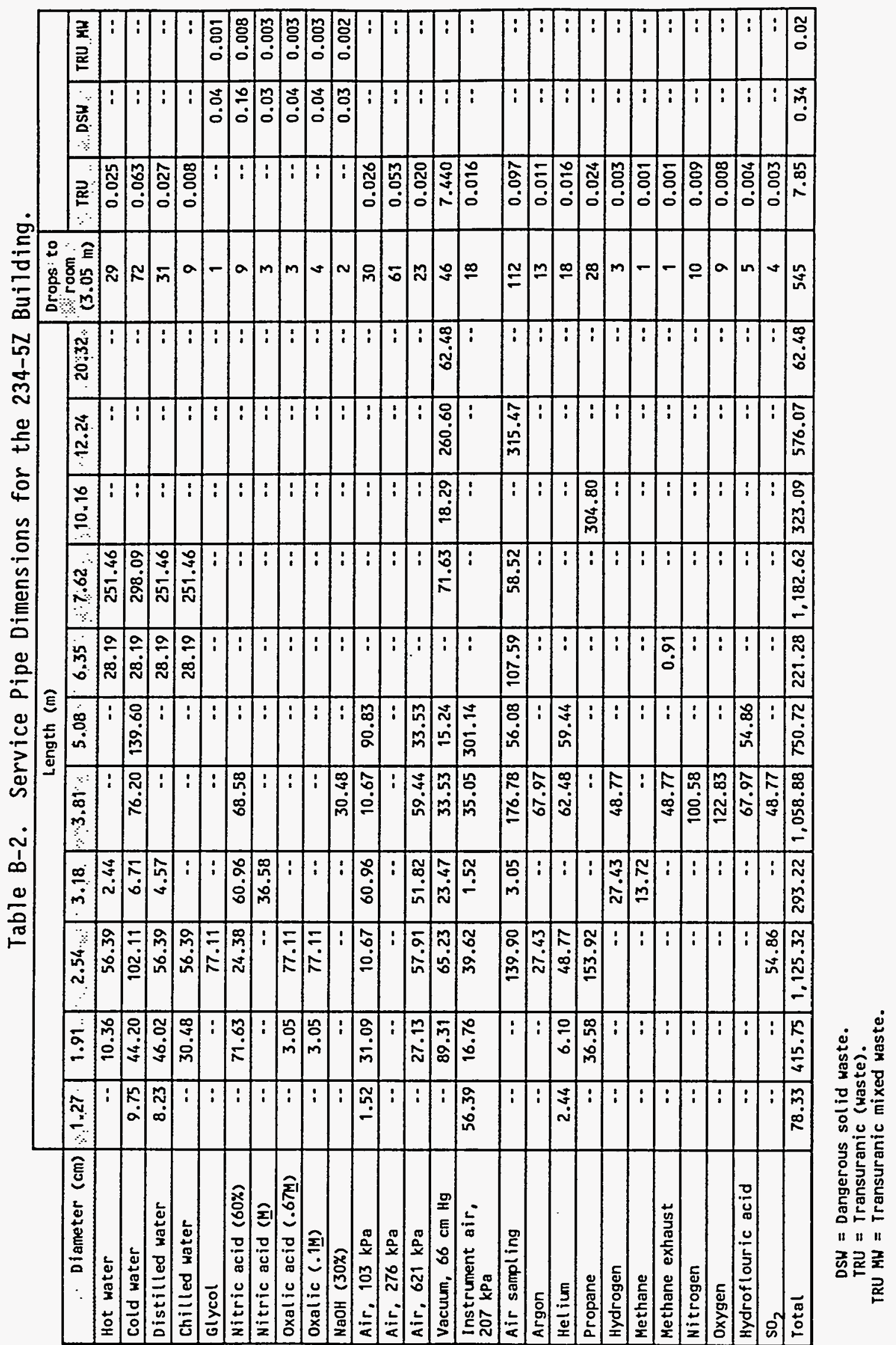


Table B-3. Service Pipe Dimensions for the 236-Z Building.

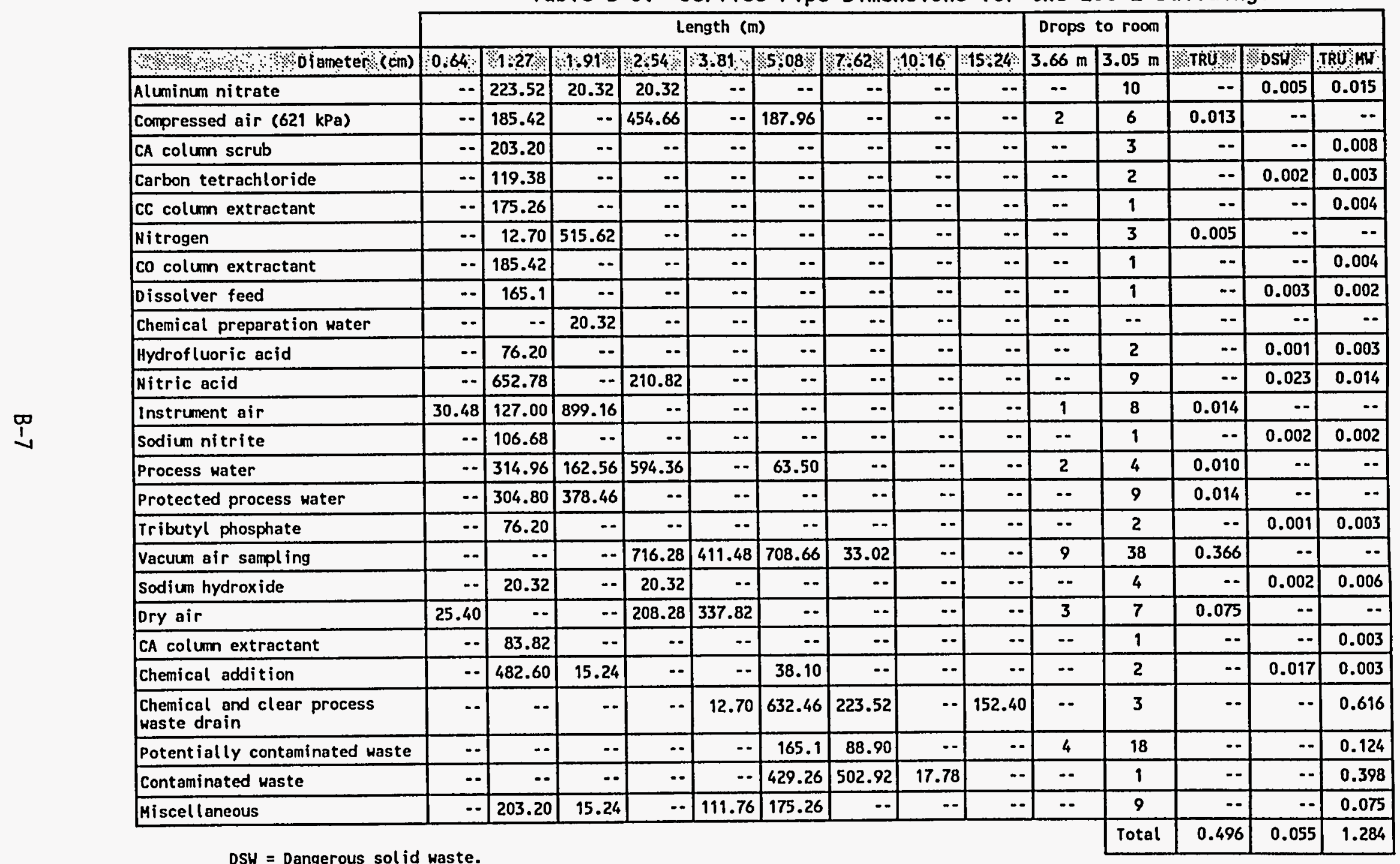

DSW = Dangerous sol id waste.

TRU = Transuranic (waste).

TRU MH = Transuranic mixed

waste. 
WHC-EP-0829

This page intentionally left blank.

B-8 
WHC-EP-0829

\section{APPENDIX C \\ ESTIMATION OF VENTILATION REMOVAL DURING DECONTAMINATION AND DECOMMISSIONING AT THE PLUTONIUM FINISHING PLANT}


WHC-EP-0829

This page intentionally left blank.

$c-2$ 


\section{ESTIMATION OF VENTILATION REHOVAL DURING DECONTAMINATION AND DECOHHISSIONING AT THE PLUTONIUM FINISHING PLANT}

This appendix contains the spreadsheet (generated in Excel 5.0) that was used to estimate the volume of duct work (and other ventilation items) that is likely to be removed during the decontamination and decommissioning of the Plutonium Finishing Plant. 
Table C-1. Air Supply System for Building 2736-ZB.

\begin{tabular}{|c|c|c|c|c|c|}
\hline \multicolumn{2}{|c|}{ Total length } & \multicolumn{2}{|c|}{ Dimensions of duct } & \multicolumn{2}{|c|}{ Volume } \\
\hline ft? & $\because-m \geqslant$ & $\therefore \ln n$ & $\because \mathrm{cm}$ & $\sin ^{3}$ & $m^{3}$ \\
\hline 22 & 6.71 & $6 \times 6$ & $15.24 \times 15.24$ & 9,504 & 0.1557 \\
\hline 6 & 1.83 & $7 \times 7$ & $17.78 \times 17.78$ & 3,528 & 0.0578 \\
\hline 87 & 26.52 & $8 \times 4$ & $20.32 \times 10.16$ & 33,408 & 0.5475 \\
\hline 20 & 6.10 & $8 \times 6$ & $20.32 \times 15.24$ & 11,520 & 0.1888 \\
\hline 15 & 4.57 & $10 \times 6$ & $25.40 \times 15.24$ & 10,800 & 0.1770 \\
\hline 67 & 20.42 & $12 \times 10$ & $30.48 \times 25.40$ & 96,480 & 1.5810 \\
\hline 85 & 25.91 & $12 \times 8$ & $30.48 \times 20.32$ & 97,920 & 1.6046 \\
\hline 104 & 31.70 & $12 \times 6$ & $30.48 \times 15.24$ & 89,856 & 1.4725 \\
\hline 39 & 11.89 & $12 \times 12$ & $30.48 \times 30.48$ & 67,392 & 1.1044 \\
\hline 57 & 17.37 & $16 \times 16$ & $40.64 \times 40.64$ & 175,104 & 2.8694 \\
\hline 12 & 3.66 & $16 \times 12$ & $40.64 \times 30.48$ & 27,648 & 0.4531 \\
\hline 6 & 1.83 & $18 \times 8$ & $45.72 \times 20.32$ & 10,368 & 0.1699 \\
\hline 12 & 3.66 & $20 \times 12$ & $50.80 \times 30.48$ & 34,560 & 0.5663 \\
\hline 17 & 5.18 & $22 \times 12$ & $55.88 \times 30.48$ & 53,856 & 0.8825 \\
\hline 34 & 10.36 & $24 \times 12$ & $60.96 \times 30.48$ & 117,504 & 1.9255 \\
\hline 48 & 14.63 & $28 \times 12$ & $71.12 \times 30.48$ & 193,536 & 3.1715 \\
\hline 104 & 31.70 & $30 \times 16$ & $76.20 \times 40.64$ & 599,040 & 9.8165 \\
\hline 85 & 25.91 & $30 \times 12$ & $76.20 \times 30.48$ & 367,200 & 6.0173 \\
\hline 77 & 23.47 & $36 \times 14$ & $91.44 \times 35.56$ & 465,696 & 7.6314 \\
\hline 36 & 10.97 & $18 \times 12$ & $45.72 \times 30.48$ & 93,312 & 1.5291 \\
\hline 38 & 11.58 & $18 \times 8$ & $45.72 \times 20.32$ & 65,664 & 1.0760 \\
\hline 18 & 5.49 & $18 \times 8$ & $45.72 \times 20.32$ & 31,104 & 0.5097 \\
\hline \multicolumn{4}{|c|}{ Total volume for air supply systems } & $2,655,000$ & 43.5075 \\
\hline
\end{tabular}


Table C-2. Exhaust System for Building 2736-ZB.

\begin{tabular}{|r|r|r|r|r|r|}
\hline \multicolumn{2}{|c|}{ Length } & \multicolumn{2}{|c|}{ Duct diameter/dimensions } & \multicolumn{2}{c|}{ Volume } \\
\hline 46 & 14.02 & 28 & 71.12 & 339,723 & 5.5670 \\
\hline 50 & 15.24 & 10 & 25.40 & 47,100 & 0.7718 \\
\hline 33 & 10.06 & $28 \times 24$ & $71.12 \times 60.96$ & 266,112 & 4.3608 \\
\hline 36 & 10.97 & $28 \times 24$ & $71.12 \times 60.96$ & 290,304 & 4.7572 \\
\hline 27 & 8.23 & 28 & 71.12 & 199,403 & 3.2676 \\
\hline 27 & 8.23 & 16 & 40.64 & 65,111 & 1.0670 \\
\hline 23 & 7.01 & 16 & 40.64 & 55,465 & 0.9089 \\
\hline 21 & 6.40 & 16 & 40.64 & 50,642 & 0.8299 \\
\hline 44 & 13.41 & $12 \times 7$ & $30.48 \times 17.78$ & 44,352 & 0.7268 \\
\hline 62 & 18.90 & $12 \times 8$ & $30.48 \times 20.32$ & 71,424 & 1.1704 \\
\hline 21 & 6.40 & $16 \times 18$ & $40.64 \times 45.72$ & 72,576 & 1.1893 \\
\hline 33 & 10.06 & $10 \times 8$ & $25.40 \times 20.32$ & 31,680 & 0.5191 \\
\hline 24 & 7.32 & 26 & 66.04 & 152,830 & 2.5044 \\
\hline 12 & 3.66 & 16 & 40.64 & 28,938 & 0.4742 \\
\hline 24 & 7.32 & 16 & 40.64 & 57,876 & 0.9484 \\
\hline 16 & 4.88 & 8 & 20.32 & 9,646 & 0.1581 \\
\hline 7 & 2.13 & 6 & 15.24 & 2,374 & 0.0389 \\
\hline 18 & 5.49 & 10 & 25.40 & 16,956 & 0.2779 \\
\hline 14 & 4.27 & 6 & 15.24 & 4,748 & 0.0778 \\
\hline 15 & 4.57 & 12 & 30.48 & 20,347 & 0.3334 \\
\hline Total volume for air supply systems & & $1,827,607$ & 29.9489 \\
\hline & & & & \\
\hline
\end{tabular}


Table C-3. E-4 Ventilation System for Building 234-5Z.

\begin{tabular}{|c|c|c|c|c|c|}
\hline \multicolumn{6}{|c|}{ Horizontal duct work } \\
\hline Total & length & \multicolumn{2}{|c|}{ Diameter/dimensions } & \multicolumn{2}{|c|}{ Volume } \\
\hline : $\mathrm{ft}^{\prime}$ & म $\mathrm{A}$ & भी & । : $\mathrm{cm}$ औ? & 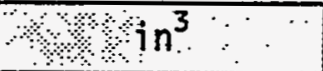 & $\therefore \mathrm{m}^{3}$ \\
\hline 15 & 4.57 & 6 & 15.24 & $5,086.8$ & 0.0834 \\
\hline 132 & 40.23 & 12 & 30.48 & $179,055.36$ & 2.9342 \\
\hline 33 & 10.06 & 14 & 35.56 & $60,928.56$ & 0.9984 \\
\hline 62 & 18.90 & 16 & 40.64 & $149,514.24$ & 2.4501 \\
\hline 10 & 3.05 & 17 & 43.18 & $27,223.8$ & 0.4461 \\
\hline 7 & 2.13 & 18 & 45.72 & $21,364.56$ & 0.3501 \\
\hline 144 & 43.89 & 20 & 50.80 & 542,592 & 8.8915 \\
\hline 27 & 8.23 & 24 & 60.96 & $146,499.84$ & 2.4007 \\
\hline 35 & 10.67 & 25 & 63.50 & $206,062.5$ & 3.3767 \\
\hline 42 & 12.80 & 30 & 76.20 & 356,076 & 5.8350 \\
\hline 30 & 9.14 & 32 & 81.28 & $289,382.4$ & 4.7421 \\
\hline 40 & 12.19 & 38 & 96.52 & $544,099.2$ & 8.9162 \\
\hline 270 & 82.30 & 42 & 106.68 & $4,486,557.6$ & 73.5212 \\
\hline 25 & 7.62 & 68 & 172.72 & $1,088,952$ & 17.8447 \\
\hline 600 & 182.88 & 12 & 30.48 & 813,888 & 13.3372 \\
\hline 50 & 15.24 & $78 \times 78$ & $198.12 \times 198.12$ & $3,650,400$ & 59.8191 \\
\hline 44 & 13.41 & $78 \times 81$ & $198.12 \times 205.74$ & $3,335,904$ & 54.6655 \\
\hline \multicolumn{4}{|c|}{ Total volume of horizontal duct work } & $15,903,586.86$ & 260.6122 \\
\hline
\end{tabular}

\begin{tabular}{|c|c|c|c|c|c|}
\hline \multicolumn{6}{|c|}{ Vertical duct work } \\
\hline \multicolumn{2}{|c|}{ Total Tength } & \multicolumn{2}{|c|}{ Diameter } & \multicolumn{2}{|c|}{ Volume } \\
\hline . $\mathrm{ft}$ & $\mathrm{m} \%$ & $\mathrm{in} \cdot \mathrm{l}$ & $\therefore \mathrm{cm} ७$ २े & $\sin ^{3}$ & $\mathrm{~m}^{3}$ \\
\hline 1,002 & 305.41 & 10 & 25.40 & 943,884 & 15.4674 \\
\hline 210 & 64.01 & 15 & 38.10 & 445,095 & 7.2938 \\
\hline \multicolumn{4}{|c|}{ Total volume of vertical duct work } & $1,388,979$ & 22.7612 \\
\hline
\end{tabular}


Tab7e C-4. Air Zone Systems for Building 234-5Z. (3 sheets)

\begin{tabular}{|c|c|c|c|c|c|}
\hline \multicolumn{6}{|c|}{ System S-2 } \\
\hline \multicolumn{2}{|c|}{ Length } & \multicolumn{2}{|c|}{ Duct dimensions } & \multicolumn{2}{|c|}{ Volume } \\
\hline $1.17 \%$ & 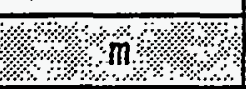 & ४४ $1 n \%$ & $1.1 \mathrm{~cm} .8$ & $1 \mathrm{n}^{3}$ & $\mathrm{pm}^{3}$ \\
\hline 25 & 7.62 & $24 \times 10$ & $60.96 \times 25.40$ & 72,000 & 1.1799 \\
\hline 10 & 3.05 & $15 \times 26$ & $38.10 \times 66.04$ & 46,800 & 0.7669 \\
\hline 20 & 6.10 & $15 \times 26$ & $38.10 \times 66.04$ & 93,600 & 1.5338 \\
\hline 75 & 22.86 & $18 \times 16$ & $45.72 \times 40.64$ & 259,200 & 4.2475 \\
\hline 25 & 7.62 & $16 \times 14$ & $40.64 \times 35.56$ & 67,200 & 1.1012 \\
\hline 40 & 12.19 & $16 \times 22$ & $40.64 \times 55.88$ & 168,960 & 2.7687 \\
\hline 220 & 67.06 & $60 \times 30$ & $152.40 \times 76.20$ & $4,752,000$ & 77.8710 \\
\hline 30 & 9.14 & $18 \times 16$ & $45.72 \times 40.64$ & 103,680 & 1.6990 \\
\hline 20 & 6.10 & $24 \times 12$ & $60.96 \times 30.48$ & 69,120 & 1.1327 \\
\hline 100 & 30.48 & $20 \times 12$ & $50.80 \times 30.48$ & 288,000 & 4.7195 \\
\hline 20 & 6.10 & $18 \times 12$ & $45.72 \times 30.48$ & 51,840 & 0.8495 \\
\hline 23 & 7.01 & $18 \times 16$ & $45.72 \times 40.64$ & 79,488 & 1.3026 \\
\hline 10 & 3.05 & $24 \times 9$ & $60.96 \times 22.86$ & 25,920 & 0.4248 \\
\hline 140 & 42.67 & $24 \times 24$ & $60.96 \times 60.96$ & 967,680 & 15.8574 \\
\hline 73 & 22.25 & $48 \times 28$ & $121.92 \times 71.12$ & $1,177,344$ & 19.2931 \\
\hline 120 & 36.58 & $48 \times 28$ & $121.92 \times 71.12$ & $1,935,360$ & 31.7147 \\
\hline 100 & 30.48 & $84 \times 48$ & $213.36 \times 121.92$ & $4,838,400$ & 79.2869 \\
\hline 95 & 28.96 & $84 \times 60$ & $213.36 \times 152.40$ & $5,745,600$ & 94.1531 \\
\hline 67 & 20.42 & $38 \times 20$ & $96.52 \times 50.80$ & 611,040 & 10.0131 \\
\hline 17 & 5.18 & $20 \times 16$ & $50.80 \times 40.64$ & 65,280 & 1.0697 \\
\hline 140 & 42.67 & $30 \times 24$ & $76.20 \times 60.96$ & $1,209,600$ & 19.8217 \\
\hline \multicolumn{4}{|c|}{ Total volume } & $22,628,112$ & 370.8068 \\
\hline
\end{tabular}


Table C-4. Air Zone Systems for Building 234-5Z. (3 sheets)

\begin{tabular}{|c|c|c|c|c|c|}
\hline \multicolumn{6}{|c|}{ System S-3A } \\
\hline \multicolumn{2}{|c|}{ Length } & \multicolumn{2}{|c|}{ Duct dimensions } & \multicolumn{2}{|c|}{ Volume } \\
\hline . $\mathrm{ft} / \mathrm{l}$ & लmध & $4.1 \mathrm{H}$ & ४ै. $\mathrm{cm} / \mathrm{l}$ & * $i n^{3}$ & $\mathrm{~m}^{3} \%$ \\
\hline 52 & 15.85 & $18 \times 32$ & $45.72 \times 81.28$ & 359,424 & 5.8899 \\
\hline 105 & 32.00 & $18 \times 16$ & $45.72 \times 40.64$ & 362,880 & 5.9465 \\
\hline 60 & 18.29 & $18 \times 16$ & $45.72 \times 40.64$ & 207,360 & 3.3980 \\
\hline 35 & 10.67 & $20 \times 24$ & $50.80 \times 60.96$ & 201,600 & 3.3036 \\
\hline 80 & 24.38 & $24 \times 24$ & $60.96 \times 60.96$ & 552,960 & 9.0614 \\
\hline 103 & 31.39 & $30 \times 24$ & $76.20 \times 60.96$ & 889,920 & 14.5831 \\
\hline 55 & 16.76 & $30 \times 66$ & $76.20 \times 167.64$ & $1,306,800$ & 21.4145 \\
\hline 67 & 20.42 & $30 \times 48$ & $76.20 \times 121.92$ & $1,157,760$ & 18.9722 \\
\hline 40 & 12.19 & $33 \times 28$ & $83.82 \times 71.12$ & 443,520 & 7.2680 \\
\hline 70 & 21.34 & $36 \times 42$ & $91.44 \times 106.68$ & $1,270,080$ & 20.8128 \\
\hline 75 & 22.86 & $42 \times 24$ & $106.68 \times 60.96$ & 907,200 & 14.8663 \\
\hline 190 & 57.91 & $48 \times 24$ & $121.92 \times 60.96$ & $2,626,560$ & 43.0414 \\
\hline 45 & 13.72 & $48 \times 42$ & $121.92 \times 106.68$ & $1,088,640$ & 17.8395 \\
\hline 30 & 9.14 & $48 \times 20$ & $121.92 \times 50.80$ & 345,600 & 5.6633 \\
\hline 50 & 15.24 & $48 \times 44$ & $121.92 \times 111.76$ & $1,267,200$ & 20.7656 \\
\hline 20 & 6.10 & $50 \times 54$ & $127.00 \times 137.16$ & 648,000 & 10.6188 \\
\hline 15 & 4.57 & $72 \times 120$ & $182.88 \times 304.80$ & $1,555,200$ & 25.4851 \\
\hline 140 & 42.67 & $96 \times 66$ & $243.84 \times 167.64$ & $10,644,480$ & 174.4311 \\
\hline 20 & 6.10 & $66 \times 28$ & $167.64 \times 71.12$ & 443,520 & 7.2680 \\
\hline 140 & 42.67 & $66 \times 24$ & $167.64 \times 60.96$ & $2,661,120$ & 43.6078 \\
\hline 160 & 48.77 & $66 \times 96$ & $167.64 \times 243.84$ & $12,165,120$ & 199.3498 \\
\hline \multicolumn{4}{|c|}{ Total volume } & $41,104,944$ & 673.5867 \\
\hline
\end{tabular}


Table C-4. Air Zone Systems for Building 234-5Z. (3 sheets)

\begin{tabular}{|c|c|c|c|c|c|}
\hline \multicolumn{6}{|c|}{ System S-3 } \\
\hline \multicolumn{2}{|c|}{ Length } & \multicolumn{2}{|c|}{ Duct dimensions } & \multicolumn{2}{|c|}{ Volume } \\
\hline \%+t/\% & $\mathrm{m} / 2$ & ২ै. & $(4) .4 \mathrm{~cm}+\%$ & $1 \% \mathrm{in}^{3}: \cdots$ & अ $\mathrm{m}^{3} \mathrm{k}$ \\
\hline 22 & 6.71 & $26 \times 16$ & $66.04 \times 40.64$ & 109,824 & 1.7997 \\
\hline 68 & 20.73 & $72 \times 30$ & $182.88 \times 76.20$ & $1,762,560$ & 28.8831 \\
\hline 67 & 20.42 & $72 \times 48$ & $182.88 \times 121.92$ & $2,778,624$ & 45.5333 \\
\hline 57 & 17.37 & $36 \times 24$ & $91.44 \times 60.96$ & 590,976 & 9.6843 \\
\hline 190 & 57.91 & $60 \times 30$ & $152.40 \times 76.20$ & $4,104,000$ & 67.2522 \\
\hline 79 & 24.08 & $54 \times 20$ & $137.16 \times 50.80$ & $1,023,840$ & 16.7777 \\
\hline 137 & 41.76 & $36 \times 20$ & $91.44 \times 50.80$ & $1,183,680$ & 19.3970 \\
\hline 132 & 40.23 & $24 \times 20$ & $60.96 \times 50.80$ & 760,320 & 12.4594 \\
\hline 115 & 35.05 & $20 \times 20$ & $50.80 \times 50.80$ & 552,000 & 9.0456 \\
\hline 117 & 35.66 & $36 \times 18$ & $91.44 \times 45.72$ & 909,792 & 14.9088 \\
\hline 113 & 34.44 & $60 \times 18$ & $152.40 \times 45.72$ & $1,464,480$ & 23.9984 \\
\hline 89 & 27.13 & $60 \times 40$ & $152.40 \times 101.60$ & $2,563,200$ & 42.0032 \\
\hline 37 & 11.28 & $24 \times 24$ & $60.96 \times 60.96$ & 255,744 & 4.1909 \\
\hline 185 & 56.39 & $90 \times 30$ & $228.60 \times 76.20$ & $5,994,000$ & 98.2237 \\
\hline 185 & 56.39 & $90 \times 48$ & $228.60 \times 121.92$ & $9,590,400$ & 157.1579 \\
\hline 85 & 25.91 & $36 \times 24$ & $91.44 \times 60.96$ & 881,280 & 14.4415 \\
\hline 12 & 3.66 & $72 \times 30$ & $182.88 \times 76.20$ & 311,040 & 5.0970 \\
\hline \multicolumn{4}{|c|}{ Total volume } & $34,835,760$ & 570.8537 \\
\hline
\end{tabular}

Total volume for air supply systems $S-2, S-3 A, S-3$ Total volume with $1 \%$ for $S-5$

\begin{tabular}{|l|l|l|}
\hline $98,568,816$ & $1,615.2472$ \\
\hline $99,554,504$ & $1,631.3996$ \\
\hline
\end{tabular}


Table C-5. E-3 Ventilation System for Building 234-5Z. (3 sheets)

\begin{tabular}{|c|c|c|c|c|c|}
\hline \multicolumn{6}{|c|}{ Horizontal ducting } \\
\hline \multicolumn{2}{|c|}{ Length } & \multicolumn{2}{|c|}{ Dimensions } & \multicolumn{2}{|c|}{ Volume } \\
\hline $\mathrm{ft}$ & 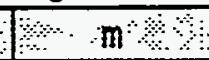 & : & अ: $: \mathrm{cm}$ & $\mathrm{ein}^{3}$ & $m^{3}$ \\
\hline 40 & 12.19 & $10 \times 10$ & $25.40 \times 25.40$ & 48,000 & 0.7866 \\
\hline 20 & 6.10 & $12 \times 30$ & $30.48 \times 76.20$ & 86,400 & 1.4158 \\
\hline 13 & 3.96 & $15 \times 11$ & $38.10 \times 27.94$ & 25,740 & 0.4218 \\
\hline 30 & 9.14 & $18 \times 74$ & $45.72 \times 187.96$ & 479,520 & 7.8579 \\
\hline 60 & 18.29 & $22 \times 22$ & $55.88 \times 55.88$ & 348,480 & 5.7105 \\
\hline 60 & 18.29 & $24 \times 34$ & $60.96 \times 86.36$ & 587,520 & 9.6277 \\
\hline 90 & 27.43 & $24 \times 57$ & $60.96 \times 144.78$ & $1,477,440$ & 24.2108 \\
\hline 195 & 59.44 & $24 \times 36$ & $60.96 \times 91.44$ & $2,021,760$ & 33.1306 \\
\hline 67 & 20.42 & $26 \times 10$ & $66.04 \times 25.40$ & 209,040 & 3.4255 \\
\hline 25 & 7.62 & $26 \times 24$ & $66.04 \times 60.96$ & 187,200 & 3.0676 \\
\hline 20 & 6.10 & $30 \times 28$ & $76.20 \times 71.12$ & 201,600 & 3.3036 \\
\hline 111 & 33.83 & $30 \times 20$ & $76.20 \times 50.80$ & 799,200 & 13.0965 \\
\hline 47 & 14.33 & $30 \times 16$ & $76.20 \times 40.64$ & 270,720 & 4.4363 \\
\hline 17 & 5.18 & $32 \times 12$ & $81.28 \times 30.48$ & 78,336 & 1.2837 \\
\hline 45 & 13.72 & $32 \times 20$ & $81.28 \times 50.80$ & 345,600 & 5.6633 \\
\hline 37 & 11.28 & $36 \times 34$ & $91.44 \times 86.36$ & 543,456 & 8.9056 \\
\hline 70 & 21.34 & $38 \times 24$ & $96.52 \times 60.96$ & 766,080 & 12.5538 \\
\hline 24 & 7.32 & $46 \times 24$ & $116.84 \times 60.96$ & 317,952 & 5.2103 \\
\hline 35 & 10.67 & $48 \times 30$ & $121.92 \times 76.20$ & 604,800 & 9.9109 \\
\hline 25 & 7.62 & $48 \times 80$ & $121.92 \times 203.20$ & $1,152,000$ & 18.8778 \\
\hline 77 & 23.47 & $54 \times 72$ & $137.16 \times 182.88$ & $3,592,512$ & 58.8705 \\
\hline 130 & 39.62 & $56 \times 24$ & $142.24 \times 60.96$ & $2,096,640$ & 34.3576 \\
\hline 102 & 31.09 & $66 \times 30$ & $167.64 \times 76.20$ & $2,423,520$ & 39.7142 \\
\hline 37 & 11.28 & $66 \times 54$ & $167.64 \times 137.16$ & $1,582,416$ & 25.9311 \\
\hline 59 & 17.98 & $66 \times 24$ & $167.64 \times 60.96$ & $1,121,472$ & 18.3776 \\
\hline 10 & 3.05 & $72 \times 24$ & $182.88 \times 60.96$ & 207,360 & 3.3980 \\
\hline 40 & 12.19 & $78 \times 54$ & $198.12 \times 137.16$ & $2,021,760$ & 33.1306 \\
\hline 25 & 7.62 & $84 \times 84$ & $213.36 \times 213.36$ & $2,116,800$ & 34.6880 \\
\hline 40 & 12.19 & $84 \times 96$ & $213.36 \times 243.84$ & $3,870,720$ & 63.4295 \\
\hline 110 & 33.53 & $96 \times 60$ & $243.84 \times 152.40$ & $7,603,200$ & 123.5936 \\
\hline 85 & 25.91 & $100 \times 24$ & $254.00 \times 60.96$ & $2,448,000$ & 40.1154 \\
\hline 20 & 6.10 & $120 \times 132$ & $304.80 \times 335.28$ & $3,801,600$ & 62.2968 \\
\hline 30 & 9.14 & $120 \times 96$ & $304.80 \times 243.84$ & $4,147,200$ & 67.9602 \\
\hline 92 & 28.04 & $132 \times 132$ & $335.28 \times 335.28$ & $19,236,096$ & 315.2219 \\
\hline 135 & 41.15 & $144 \times 120$ & $365.76 \times 304.80$ & $27,993,600$ & 458.7311 \\
\hline 65 & 19.81 & $144 \times 96$ & $365.76 \times 243.84$ & $10,782,720$ & 176.6964 \\
\hline & Total v & lume horizor & 1 ducting & $105,596,460$ & $1,729.4091$ \\
\hline
\end{tabular}




\begin{tabular}{|c|c|c|c|c|}
\hline$D I Z \varepsilon^{\circ} I$ & $079^{6} 08$ & $99^{\circ} 9 \varepsilon \times 96^{\circ} 09$ & $t I \times b z$ & 2 \\
\hline SOEL I & $009^{6} \mathrm{SOI}$ & $2 \varepsilon^{\circ} 02 \times 88^{\circ} 59$ & $8 \times 22$ & $\mathbf{g}$ \\
\hline $2998^{\circ} 0$ & $008^{\circ} 29$ & $08^{\circ} 09 \times 88^{\circ} 99$ & $02 \times 22$ & I \\
\hline $292 \varepsilon^{\circ} 7$ & $000^{\circ} \div 92$ & $00^{\circ} 92 \times 88^{\circ} 99$ & $0[\times 22$ & OI \\
\hline $998 L^{\circ} 0$ & $000^{\prime} 8 t$ & $08^{\circ} 09 \times 08^{\circ} 09$ & $02 \times 02$ & $I$ \\
\hline $998 L^{\circ} 0$ & $000^{6} 8 t$ & $00^{\circ} 92 \times 08^{\circ} 09$ & $0[\times 02$ & 2 \\
\hline $0 \forall \subseteq \varepsilon^{\circ} 0$ & $009^{6}[2$ & $98^{\circ} 22 \times 08^{\circ} 09$ & $6 \times 02$ & $I$ \\
\hline $0+9 \varepsilon^{\circ} 0$ & $009^{6} \tau 2$ & $0 b^{\circ} G Z \times 2 L^{\circ} s t$ & $0[\times 8 \mathrm{I}$ & $\bar{I}$ \\
\hline $2 \varepsilon 82 \cdot 0$ & $082^{6} \angle I$ & $2 \varepsilon^{\circ} 02 \times 2 L^{\circ} s t$ & $8 \times 8 I$ & $\bar{I}$ \\
\hline$\varepsilon 899^{\circ} 0$ & $089^{6} t \varepsilon$ & $8 I^{\circ} \varepsilon t \times 8 I^{\circ} \varepsilon t$ & $\angle I \times \angle I$ & I \\
\hline ISGL 0 & $080^{\circ} 9 b$ & $8 t^{\circ} 0 \varepsilon \times+9^{\circ} 0 t$ & $2 I \times 9 I$ & 2 \\
\hline$\nabla \varepsilon 0 \mathcal{G}^{\circ} 0$ & $0 Z L^{6} 0 \varepsilon$ & $79^{\circ} 0 t \times+9^{\circ} 0 t$ & $91 \times 91$ & I \\
\hline ISS $L^{\circ} 0$ & $080^{\prime} 9 t$ & $2 \varepsilon^{\circ} 02 \times+9^{\circ} 0 t$ & $8 \times 9[$ & $\varepsilon$ \\
\hline$\varepsilon 629^{\circ} 0$ & $00 t^{6} 8 \varepsilon$ & $0 t^{\circ} 92 \times+9^{\circ} 0 t$ & OI $\times 9 I$ & 2 \\
\hline $6689^{\circ} 0$ & $000^{\circ} 9 \varepsilon$ & $08^{\circ} 09 \times 0 I^{\circ} 8 \varepsilon$ & $02 \times S I$ & $I$ \\
\hline$t 2 t t \cdot 0$ & $000^{\prime} \mathrm{LI}$ & $0 \tau^{\circ} 8 \varepsilon \times 0{ }^{\circ} 8 \varepsilon$ & $\mathcal{G I \times G I}$ & I \\
\hline$S \pitchfork z \varepsilon^{\circ} 0$ & $008^{6} 6 I$ & $76^{\circ} \angle 2 \times 0 I^{\circ} 8 \varepsilon$ & II $\times$ II & I \\
\hline $80 \angle L^{\circ} 0$ & $0 \rightarrow 0^{\circ} \angle t$ & $99^{\circ} \varsigma \varepsilon \times 99^{\circ} \mathcal{G}$ & $\forall[\times \forall[$ & 2 \\
\hline$\angle 26 I^{\circ} 0$ & $09 L^{6} I I$ & $8 L^{\circ} \angle\left[\times 99^{\circ} G \varepsilon\right.$. & $L X \nabla I$ & $I$ \\
\hline$\angle t 99^{\circ} 0$ & $099^{\circ} 0 t$ & $20^{\circ} \varepsilon \varepsilon \times 20^{\circ} \varepsilon \varepsilon$ & $\varepsilon I \times \varepsilon I$ & 2 \\
\hline$\varepsilon 999^{\circ} 0$ & $09 S^{6} \nabla \varepsilon$ & $8 t^{\circ} 0 \varepsilon \times 8 t^{\circ} 0 \varepsilon$ & $2 I \times 2 I$ & 2 \\
\hline $888 \mathrm{I}^{\circ} 0$ & $02 S^{6} \mathrm{II}$ & $2 \varepsilon^{\circ} 02 \times 87^{\circ} 0 \varepsilon$ & $8 \times 2 I$ & $I$ \\
\hline $6 \angle O L \cdot 0$ & $00 z^{6} \varepsilon t$ & $00^{\circ} 92 \times 80^{\circ} 0 \varepsilon$ & OI $\times 2 \mathrm{II}$ & $\varepsilon$ \\
\hline $2 \varepsilon 82^{\circ} 0$ & $082^{6} \angle I$ & $t \tau^{\circ} 9\left[\times 8 t^{\circ} 0 \varepsilon\right.$ & $9 \times 2 I$ & 2 \\
\hline $8\left[96^{\circ} 0\right.$ & $080^{\prime} 89$ & $66^{\circ} \angle 2 \times 66^{\circ} \angle 2$ & II $\times$ II & $t$ \\
\hline $2 \varepsilon 86^{\circ} 0$ & $000^{6} 09$ & $0 t^{\circ} 92 \times 0 t^{\circ} 92$ & OI $\times 0 I$ & $\mathrm{~s}$ \\
\hline$\varepsilon 629^{\circ} 0$ & $00 t^{\prime} 8 \varepsilon$ & $2 \varepsilon^{\circ} 02 \times 0 t^{\circ} s z$ & $8 \times 0 \mathrm{I}$ & $t$ \\
\hline $692 I^{\circ} 0$ & $089^{\circ} \mathrm{L}$ & $2 \varepsilon^{\circ} 02 \times 2 \varepsilon^{\circ} 02$ & $8 \times 8$ & $\bar{I}$ \\
\hline$\varepsilon 6 \mathrm{SI}^{\circ} 0$ & $02 L^{6} 6$ & $98^{\circ} 22 \times 98^{\circ} 22$ & $6 \times 6$ & $I$ \\
\hline $6660^{\circ} 0$ & $09 L^{6} \div 9$ & $\nabla 2^{\circ} 9 I \times 2 \varepsilon^{\circ} 02$ & $9 \times 8$ & $I$ \\
\hline$\angle 26 I^{\circ} 0$ & $09 L^{\prime} I I$ & $8 L^{\circ} \angle I \times 8 L^{\circ} \angle I$ & $\angle \times L$ & 2 \\
\hline $9280^{\circ} 0$ & $0+0^{\circ} \mathrm{s}$ & $b Z^{\circ} G I \times 8 L^{\circ} \angle I$ & $9 \times 1$ & $I$ \\
\hline $2 \varepsilon 82 \cdot 0$ & $082^{6} \angle I$ & $\forall Z^{\circ} G I \times \nabla Z^{\circ} G I$ & $9 \times 9$ & 7 \\
\hline $2 \varepsilon 82^{\circ} 0$ & $082^{6} \angle I$ & $86^{\circ} 0 \varepsilon \times \$ 2^{\circ} G[$ & $21 \times 9$ & 2 \\
\hline$\varepsilon \angle \mathcal{E} T^{\circ} 0$ & $009^{6} 6$ & $2 \varepsilon^{\circ} 02 \times 0 L^{\circ} 2 I$ & $8 \times 9$ & 2 \\
\hline ४. \& & $\because{ }_{\mathrm{S}} \mathrm{ul} \%$ & \% " แगे।? & \% 40 & (1) 0 L $)$ W $90 \%$ \\
\hline \multicolumn{2}{|c|}{$\frac{1}{\tan [0 \Lambda}$} & \multicolumn{2}{|c|}{ suo! suaw! 0} & sqonp to laquinN \\
\hline & & 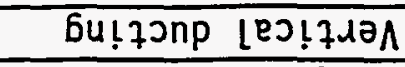 & & \\
\hline
\end{tabular}

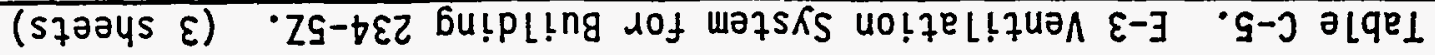


Table C-5. E-3 Ventilation System for Building 234-5Z. (3 sheets)

\begin{tabular}{|c|c|c|c|c|}
\hline \multicolumn{5}{|c|}{ Vertical ducting } \\
\hline Number of ducts & \multicolumn{2}{|c|}{ Dimensions } & \multicolumn{2}{|c|}{ Volume } \\
\hline $3.05 \mathrm{~m} /(10 \mathrm{ft})$ & न $\ln$ \% & ৫ $\mathrm{cm}$ & Q $\operatorname{in}^{3} \mathrm{~s}$ & $\mathrm{~m}^{3}$ \\
\hline 1 & $24 \times 8$ & $60.96 \times 20.32$ & 23,040 & 0.3776 \\
\hline $\bar{I}$ & $24 \times 12$ & $60.96 \times 30.48$ & 34,560 & 0.5663 \\
\hline 1 & $24 \times 24$ & $60.96 \times 60.96$ & 69,120 & 1.1327 \\
\hline 1 & $24 \times 15$ & $60.96 \times 38.10$ & 43,200 & 0.7079 \\
\hline 3 & $26 \times 6$ & $66.04 \times 15.24$ & 56,160 & 0.9203 \\
\hline 58 & $26 \times 10$ & $66.04 \times 25.40$ & $1,809,600$ & 29.6539 \\
\hline$\overline{1}$ & $27 \times 10$ & $68.58 \times 25.40$ & 32,400 & 0.5309 \\
\hline 1 & $30 \times 5$ & $76.20 \times 12.70$ & 18,000 & 0.2950 \\
\hline 3 & $30 \times 8$ & $76.20 \times 20.32$ & 86,400 & 1.4158 \\
\hline 2 & $30 \times 10$ & $76.20 \times 25.40$ & 72,000 & 1.1799 \\
\hline 4 & $30 \times 12$ & $76.20 \times 30.58$ & 172,800 & 2.8317 \\
\hline$\overline{1}$ & $32 \times 7$ & $81.28 \times 17.78$ & 26,880 & 0.4405 \\
\hline 5 & $36 \times 10$ & $91.44 \times 25.40$ & 216,000 & 3.5396 \\
\hline 1 & $44 \times 10$ & $111.76 \times 25.40$ & 52,800 & 0.8652 \\
\hline \multicolumn{3}{|c|}{ Total volume vertical ducting } & $4,097,760$ & 67.1503 \\
\hline
\end{tabular}


Table C-6. Air Support system for Building 236-Z. (2 sheets)

\begin{tabular}{|c|c|c|c|c|c|c|}
\hline \multirow[b]{2}{*}{ Floor } & \multicolumn{2}{|c|}{ Total Tength } & \multicolumn{2}{|c|}{ Duct dimensions } & \multicolumn{2}{|c|}{ Volume } \\
\hline & f $t$ & m. & $1.1 \mathrm{n} .1 \%$ & 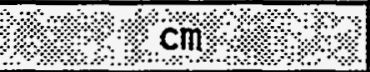 & ४ै. $\ln ^{3} \mathrm{~s}$ & $\because \quad m^{3}$ \\
\hline \multirow[t]{9}{*}{1} & 15 & 4.57 & $6 \times 6$ & $15.24 \times 15.24$ & 6,480 & 0.1062 \\
\hline & 30 & 9.14 & $10 \times 8$ & $25.40 \times 20.32$ & 28,800 & 0.4719 \\
\hline & 6 & 1.83 & $10 \times 16$ & $25.40 \times 40.64$ & 11,520 & 0.1888 \\
\hline & 24 & 7.32 & $14 \times 12$ & $35.56 \times 30.48$ & 48,384 & 0.7929 \\
\hline & 13 & 3.96 & $18 \times 12$ & $45.72 \times 30.48$ & 33,696 & 0.5522 \\
\hline & 15 & 4.57 & $18 \times 18$ & $45.72 \times 45.72$ & 58,320 & 0.9557 \\
\hline & 164 & 49.99 & $24 \times 16$ & $60.96 \times 40.64$ & 755,712 & 12.3839 \\
\hline & 19 & 5.79 & $26 \times 18$ & $66.04 \times 45.72$ & 106,704 & 1.7486 \\
\hline & 13 & 3.96 & $30 \times 18$ & $76.20 \times 45.72$ & 84,240 & 1.3804 \\
\hline \multirow[t]{4}{*}{2} & 12 & 3.66 & $13 \times 13$ & $33.02 \times 33.02$ & 24,336 & 0.3988 \\
\hline & 9 & 2.74 & $14 \times 12$ & $35.56 \times 30.48$ & 18,144 & 0.2973 \\
\hline & 131 & 39.93 & $24 \times 16$ & $60.96 \times 40.64$ & 603,648 & 9.8920 \\
\hline & 38 & 11.58 & $24 \times 12$ & $60.96 \times 30.48$ & 131,328 & 2.1521 \\
\hline \multirow[t]{9}{*}{3} & 10 & 3.05 & $8 \times 8$ & $20.32 \times 20.32$ & 7,680 & 0.1259 \\
\hline & 24 & 7.32 & $10 \times 24$ & $25.40 \times 60.96$ & 69,120 & 1.1327 \\
\hline & 4 & 1.22 & $10 \times 8$ & $25.40 \times 20.32$ & 3,840 & 0.0629 \\
\hline & 37 & 11.28 & $14 \times 12$ & $35.56 \times 30.48$ & 74,592 & 1.2223 \\
\hline & 7 & 2.13 & $14 \times 10$ & $35.56 \times 25.40$ & 11,760 & 0.1927 \\
\hline & 21 & 6.40 & $16 \times 18$ & $40.64 \times 45.72$ & 72,576 & 1.1893 \\
\hline & 40 & 12.19 & $18 \times 30$ & $45.72 \times 76.20$ & 259,200 & 4.2475 \\
\hline & 130 & 39.62 & $20 \times 16$ & $50.80 \times 40.64$ & 499,200 & 8.1804 \\
\hline & 12 & 3.66 & $30 \times 26$ & $76.20 \times 66.04$ & 112,320 & 1.8406 \\
\hline \multirow[t]{7}{*}{4} & 9 & 2.74 & $13 \times 13$ & $33.02 \times 33.02$ & 18,252 & 0.2991 \\
\hline & 44 & 13.41 & $14 \times 22$ & $35.56 \times 55.88$ & 162,624 & 2.6649 \\
\hline & 22 & 6.71 & $14 \times 32$ & $35.56 \times 81.28$ & 118,272 & 1.9381 \\
\hline & 70 & 21.34 & $16 \times 10$ & $40.64 \times 25.40$ & 134,400 & 2.2023 \\
\hline & 9 & 2.74 & $18 \times 12$ & $45.72 \times 30.48$ & 23,328 & 0.3823 \\
\hline & 49 & 14.94 & $20 \times 16$ & $50.80 \times 40.64$ & 188,160 & 3.0834 \\
\hline & 11 & 3.35 & $20 \times 12$ & $50.80 \times 30.48$ & 31,680 & $0.519]$ \\
\hline
\end{tabular}


Table C-6. Air Support System for Building 236-Z. (2 sheets)

\begin{tabular}{|c|c|c|c|c|c|c|}
\hline & Total & Tength & \multicolumn{2}{|c|}{ Duct dimensions } & \multicolumn{2}{|c|}{ Volume } \\
\hline Floor & $\mathrm{ft}$ & की & १in. & ৪ै: cm & $\operatorname{lin}^{3} \%$ & $: \mathrm{m}^{3} \mathrm{e}$ \\
\hline \multirow{10}{*}{$\begin{array}{c}4 \\
\text { (cont.) }\end{array}$} & 40 & 12.19 & $20 \times 18$ & $50.80 \times 45.72$ & 172,800 & 2.8317 \\
\hline & 19 & 5.79 & $24 \times 12$ & $60.96 \times 30.48$ & 65,664 & 1.0760 \\
\hline & 24 & 7.32 & $24 \times 6$ & $60.96 \times 15.24$ & 41,472 & 0.6796 \\
\hline & 32 & 9.75 & $30 \times 18$ & $76.20 \times 45.72$ & 207,360 & 3.3980 \\
\hline & 4 & 1.22 & $36 \times 12$ & $91.44 \times 30.48$ & 20,736 & 0.3398 \\
\hline & 11 & 3.35 & $36 \times 18$ & $91.44 \times 45.72$ & 85,536 & 1.4017 \\
\hline & 37 & 11.28 & $40 \times 28$ & $101.60 \times 71.12$ & 497,280 & 8.1489 \\
\hline & 10 & 3.05 & $42 \times 24$ & $106.68 \times 60.96$ & 120,960 & 1.9822 \\
\hline & 6 & 1.83 & $42 \times 12$ & $106.68 \times 30.48$ & 36,288 & 0.5947 \\
\hline & 37 & 11.28 & $42 \times 42$ & $106.68 \times 106.68$ & 783,216 & 12.8346 \\
\hline 5 & 34 & 10.36 & $14 \times 22$ & $35.56 \times 55.88$ & 125,664 & 2.0593 \\
\hline \multirow[t]{3}{*}{6} & 28 & 8.53 & $14 \times 22$ & $35.56 \times 55.88$ & 103,488 & 1.6959 \\
\hline & 16 & 4.88 & $28 \times 14$ & $71.12 \times 35.56$ & 75,264 & 1.2334 \\
\hline & 9 & 2.74 & $18 \times 14$ & $45.72 \times 35.56$ & 27,216 & 0.4460 \\
\hline \multicolumn{4}{|c|}{ Total volume } & & $6,061,260$ & 99.3261 \\
\hline
\end{tabular}




\section{DISTRIBUTION}

Number of copies

OFFSITE

4

ONSITE

3

18
Los Al amos Technical Associates

8633 Gage BouTevard

Kennewick, WA 99336

J. S. Millar

T. W. Hahn

J. A. Pottmeyer

T. J. Stratton
U.S. Department of Energy-

Richland Operations Office

R. M. Gordon

R. F. Guercia

Public Reading Room

Westinghouse Hanford Company

J. D. Anderson

A. B. Carlson

J. A. Demiter

D. R. Duncan

K. L. HI adek

M. M. McCarthy

R. J. Roberts

K. J. Templeton

o. J. Valero Central Files

Information Release Administration

PNL Technical Files
S7-55

S7-55

$\mathrm{H} 2-53$
T3-04

H5-33

L5-31

H5-33

H5-33

N3-13

N3-13

K7-94

H5-33

L8-04

(3)

L8-07

P8-55 
WHC-EP-0829

This page intentionally left blank.

Distr-2 